\title{
Discovery of the Potent, Selective, Orally available CXCR7 Antagonist
}

\author{
ACT-1004-1239
}

Sylvia Richard-Bildstein*, Hamed Aissaoui, Julien Pothier, Gabriel Schäfer, Carmela

Gnerre, Eleanor Lindenberg, François Lehembre, Laetitia Pouzol, and Philippe Guerry*

Drug Discovery Chemistry, Idorsia Pharmaceuticals Ltd., Hegenheimermattweg 91, CH4123 Allschwil, Switzerland

\section{Content}

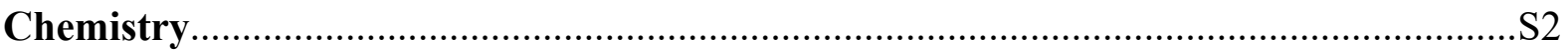

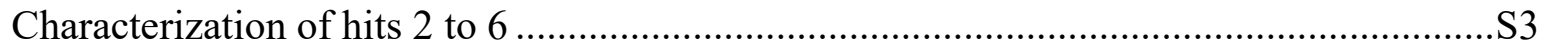

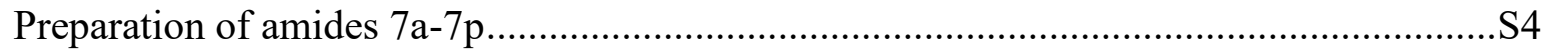

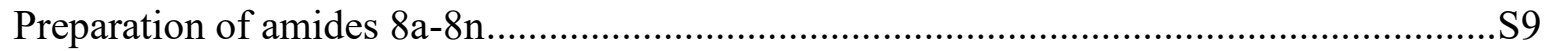

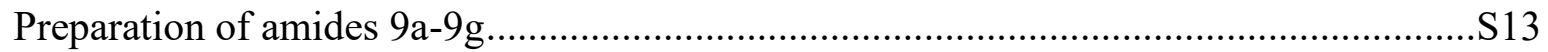

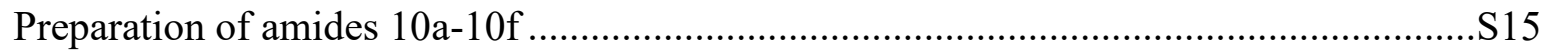

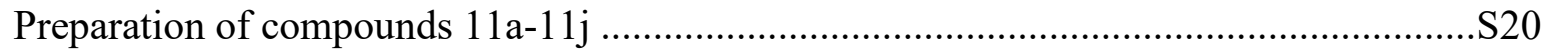

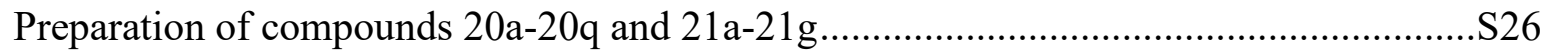

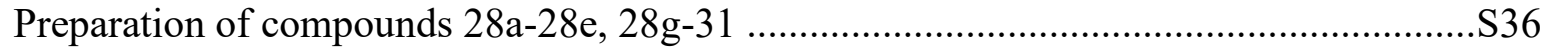

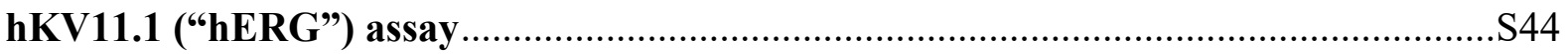

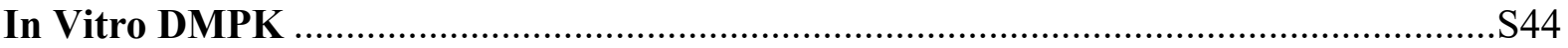

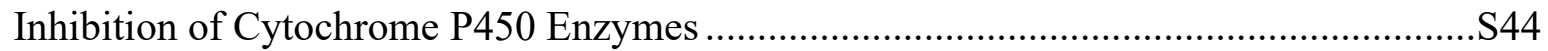

Metabolic Stability in Liver Microsomes....................................................................S44

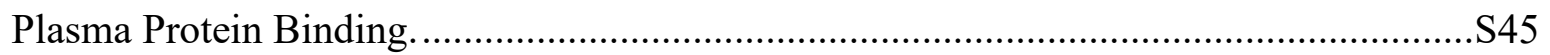

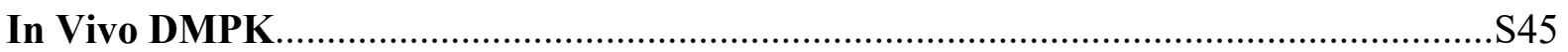

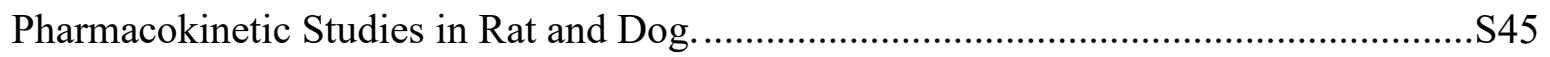

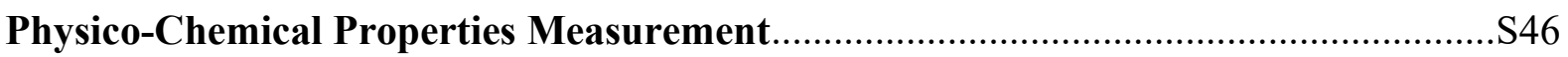




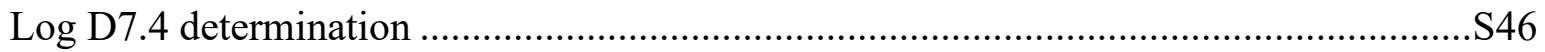

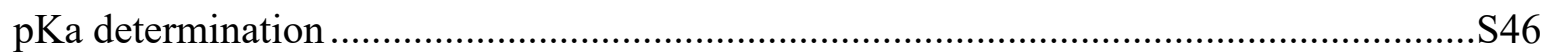

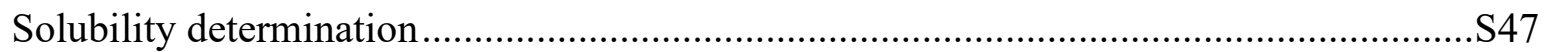

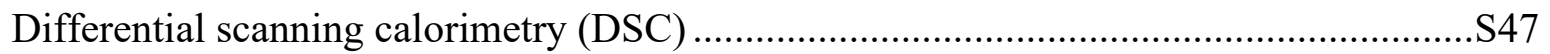

Single Crystal X-ray Structure Analysis of ACT-1004-1239 (28f) ................................S47

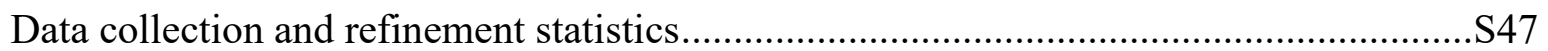

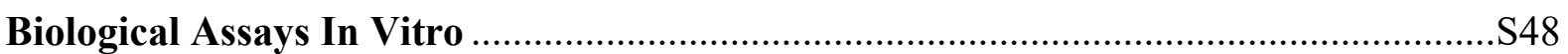

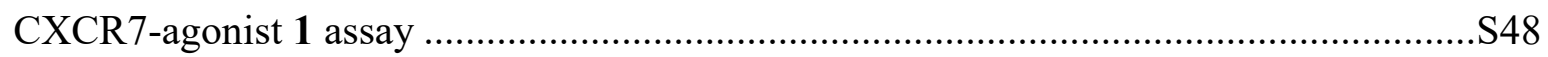

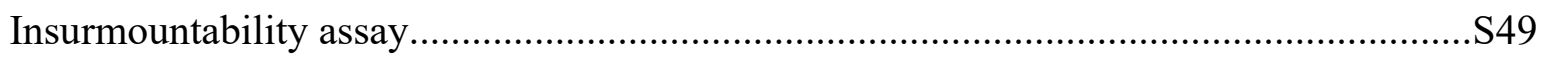

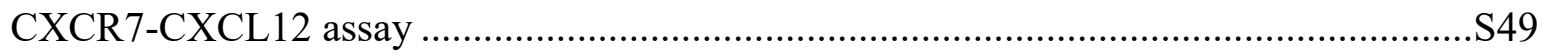

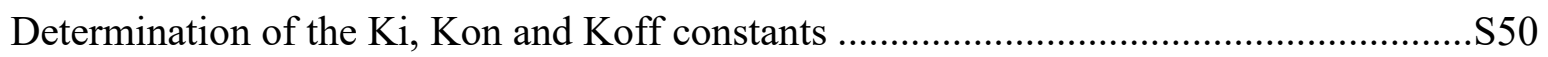

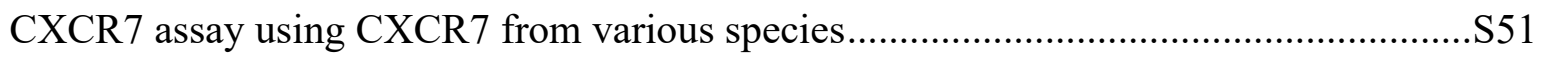

Human CXCR4 intracellular calcium liberation (FLIPR) Assay....................................S52

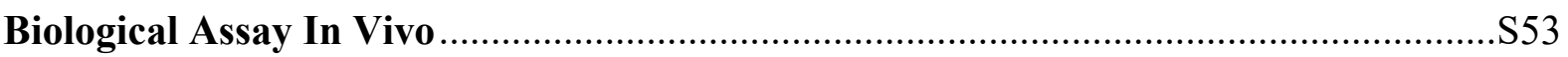

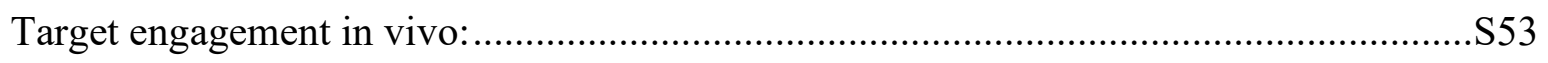

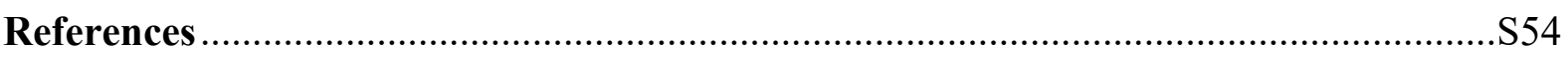

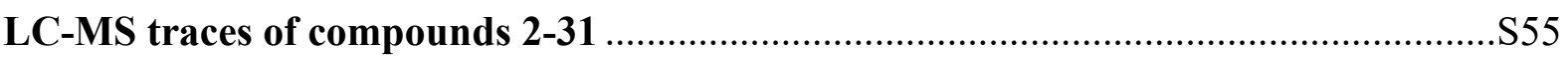

\section{Chemistry}

This supporting information contains experimental details of the synthesis of target compounds and the corresponding building blocks not described in the main text. More details on the synthesis of some derivatives discussed in this account are described in a patent application ${ }^{1}$. LC-MS and NMR equipment and methods are described in the main text. In case NMR spectra were measured using $1 \mathrm{~mm}$ Microprobe ${ }^{\circledR}$ tubes, the compounds were dissolved in nondeuterated DMSO. The spectra were then measured with double irradiation for suppression of the DMSO (2.5 ppm) and $\mathrm{H}_{2} \mathrm{O}(3.5 \mathrm{ppm})$ peaks. The number of protons given in the description represent observed values. In some cases, signals close to either of the two solvent signals were not visible as they were suppressed by irradiation. In cases where compounds appear as a 
mixture of conformational isomers, tautomers or rotamers particularly visible in their LC-MS spectra, the retention time of the most abundant conformer is given.

\section{Characterization of hits 2 to 6}

Rac-1-p-Tolyl-1H-[1,2,3]triazole-4-carboxylic acid (2-diethylamino-2-thiophen-3-ylethyl)-amide (2) QC LC-MS: $t_{R}=0.65 \mathrm{~min} ;[\mathrm{M}+\mathrm{H}]^{+}=384.3 . \mathrm{LC}-\mathrm{HRMS}: \mathrm{t}_{\mathrm{R}}=0.57 \mathrm{~min} ; \mathrm{m} / \mathrm{z}$ $=383.1780$, found $=384.1860[\mathrm{M}+\mathrm{H}]^{+} .{ }^{1} \mathrm{H}$ NMR $\left(400 \mathrm{MHz}, \mathrm{D}_{6}-\mathrm{DMSO}\right) \delta: 9.25(\mathrm{~s}, 1 \mathrm{H})$, $8.33(\mathrm{t}, J=5.0 \mathrm{~Hz}, 1 \mathrm{H}), 7.85(\mathrm{~d}, J=8.0 \mathrm{~Hz}, 2 \mathrm{H}), 7.49(\mathrm{~d}, J=3.7 \mathrm{~Hz}, 1 \mathrm{H}), 7.42(\mathrm{~m}, 3 \mathrm{H})$, $7.13(\mathrm{~d}, J=4.9 \mathrm{~Hz}, 1 \mathrm{H}), 4.20(\mathrm{t}, J=7.5 \mathrm{~Hz}, 1 \mathrm{H}), 3.64-3.73(\mathrm{~m}, 2 \mathrm{H}), 2.61-2.70(\mathrm{~m}, 2 \mathrm{H})$, $2.39(\mathrm{~s}, 3 \mathrm{H}), 2.21(\mathrm{~m}, 2 \mathrm{H}), 1.01(\mathrm{~m}, 6 \mathrm{H})$.

\section{Rac-4-(2,4-Difluoro-phenyl)-N-(2-dimethylamino-2-thiophen-3-yl-ethyl)-4-oxo-}

butyramide (3) QC LC-MS: $t_{\mathrm{R}}=0.50 \mathrm{~min} ;[\mathrm{M}+\mathrm{H}]^{+}=367.1$. LC-HRMS: $\mathrm{t}_{\mathrm{R}}=0.50 \mathrm{~min} ; \mathrm{m} / \mathrm{z}=$ 366.1214 , found $=367.1279[\mathrm{M}+\mathrm{H}]^{+} .{ }^{1} \mathrm{H}$ NMR (500 MHz, D $\left.-\mathrm{DMSO}\right) \delta: 8.88(\mathrm{~s}, 1 \mathrm{H}), 7.84$ $\left(\mathrm{td}, J_{1}=9.2 \mathrm{~Hz}, J_{2}=6.9 \mathrm{~Hz}, 1 \mathrm{H}\right), 7.50\left(\mathrm{dd}, J_{1}=4.9 \mathrm{~Hz}, J_{2}=2.9 \mathrm{~Hz}, 1 \mathrm{H}\right), 7.40\left(\mathrm{dd}, J_{1}=2.8\right.$ $\left.\mathrm{Hz}, J_{2}=1.1 \mathrm{~Hz}, 1 \mathrm{H}\right), 7.30\left(\mathrm{ddd}, J_{1}=11.7 \mathrm{~Hz}, J_{2}=9.2 \mathrm{~Hz}, J_{3}=2.5 \mathrm{~Hz}, 1 \mathrm{H}\right), 7.16\left(\mathrm{td}, J_{1}=8.3\right.$ $\left.\mathrm{Hz}, J_{2}=2.3 \mathrm{~Hz}, 1 \mathrm{H}\right), 7.01\left(\mathrm{dd}, J_{1}=4.9 \mathrm{~Hz}, J_{2}=1.2 \mathrm{~Hz}, 1 \mathrm{H}\right), 3.69\left(\mathrm{dd}, J_{1}=11.0 \mathrm{~Hz}, J_{2}=2.3\right.$ $\mathrm{Hz}, 1 \mathrm{H}), 3.61\left(\mathrm{dd}, J_{1}=14.8 \mathrm{~Hz}, J_{2}=2.8 \mathrm{~Hz}, 1 \mathrm{H}\right), 3.01\left(\mathrm{dd}, J_{1}=14.8 \mathrm{~Hz}, J_{2}=11.1 \mathrm{~Hz}, 1 \mathrm{H}\right)$, 2.59-2.66 (m, $1 \mathrm{H}), 2.37-2.43(\mathrm{~m}, 1 \mathrm{H}), 2.18-2.23(\mathrm{~m}, 2 \mathrm{H}), 1.91(\mathrm{~s}, 6 \mathrm{H})$.

Rac-4-(2,4-Difluoro-phenyl)-N-(4-methyl-morpholin-2-ylmethyl)-4-oxo-butyramide

QC LC-MS: $\mathrm{t}_{\mathrm{R}}=0.38 \mathrm{~min} ;[\mathrm{M}+\mathrm{H}]^{+}=237.2 . \mathrm{LC}-\mathrm{HRMS}: \mathrm{t}_{\mathrm{R}}=0.38 \mathrm{~min} ; \mathrm{m} / \mathrm{z}=326.1442$, found $=327.1524[\mathrm{M}+\mathrm{H}]^{+} .{ }^{1} \mathrm{H}$ NMR $\left(400 \mathrm{MHz}, \mathrm{D}_{6}-\mathrm{DMSO}\right) \delta: 7.99(\mathrm{t}, J=5.3 \mathrm{~Hz}, 1 \mathrm{H}), 7.92(\mathrm{q}, J$ $=7.7 \mathrm{~Hz}, 1 \mathrm{H}), 7.42(\mathrm{~m}, 1 \mathrm{H}), 7.24(\mathrm{t}, J=8.5 \mathrm{~Hz}, 1 \mathrm{H}), 3.76(\mathrm{~d}, J=11.2 \mathrm{~Hz}, 1 \mathrm{H}), 3.47(\mathrm{~m}, 1$ H), $3.41(\mathrm{~m}, 1 \mathrm{H}), 3.15(\mathrm{~m}, 2 \mathrm{H}), 3.08(\mathrm{t}, J=5.8 \mathrm{~Hz}, 2 \mathrm{H}), 2.64(\mathrm{~d}, J=11.2 \mathrm{~Hz}, 1 \mathrm{H}), 2.56(\mathrm{~d}$, 
$J=11.4 \mathrm{~Hz}, 1 \mathrm{H}), 2.47(\mathrm{~m}, 2 \mathrm{H}), 2.15(\mathrm{~s}, 3 \mathrm{H}), 1.93\left(\mathrm{td}, J_{1}=2.3 \mathrm{~Hz}, J_{2}=11.1 \mathrm{~Hz}, 1 \mathrm{H}\right), 1.64$ $(\mathrm{t}, J=10.5 \mathrm{~Hz}, 1 \mathrm{H})$.

\section{1-(2,4-Difluoro-phenyl)-4-[4-(2-dimethylamino-ethyl)-piperazin-1-yl]-butane-1,4-dione}

(5) QC LC-MS: $t_{R}=0.68 \mathrm{~min} ;[\mathrm{M}+\mathrm{H}]^{+}=354.0$. LC-HRMS: $t_{R}=0.39 \mathrm{~min} ; \mathrm{m} / \mathrm{z}=353.1915$, found $=354.1999[\mathrm{M}+\mathrm{H}]^{+} .{ }^{1} \mathrm{H}$ NMR $\left(400 \mathrm{MHz}, \mathrm{D}_{6}-\mathrm{DMSO}\right) \delta: 7.92(\mathrm{q}, J=8.1 \mathrm{~Hz}, 1 \mathrm{H}), 7.42$ (t, $J=11.0 \mathrm{~Hz}, 1 \mathrm{H}), 7.24(\mathrm{t}, J=8.5 \mathrm{~Hz}, 1 \mathrm{H}), 3.47(\mathrm{~m}, 2 \mathrm{H}), 3.40(\mathrm{~m}, 2 \mathrm{H}), 3.13(\mathrm{~s}, 2 \mathrm{H}), 2.69$ (t, $J=6.0 \mathrm{~Hz}, 2 \mathrm{H}), 2.38-2.43(\mathrm{~m}, 4 \mathrm{H}), 2.31-2.36(\mathrm{~m}, 4 \mathrm{H}), 2.14(\mathrm{~s}, 6 \mathrm{H})$.

\section{5-(4-Bromo-phenyl)-isoxazol-3-yl]-(8-ethyl-1-oxa-4,8-diaza-spiro[4.5]dec-4-yl)-} methanone (6) QC LC-MS: $t_{R}=0.72 \mathrm{~min} ;[\mathrm{M}+\mathrm{H}]^{+}=422.3 . \mathrm{LC}-\mathrm{HRMS}: \mathrm{t}_{\mathrm{R}}=0.64 \mathrm{~min} ; \mathrm{m} / \mathrm{z}=$ 419.0845, found $=420.0924[\mathrm{M}+\mathrm{H}]^{+} .{ }^{1} \mathrm{H} \mathrm{NMR}\left(500 \mathrm{MHz}, \mathrm{D}_{6}-\mathrm{DMSO}\right) \delta: 7.90(\mathrm{~m}, 2 \mathrm{H}), 7.78$ (m, $2 \mathrm{H}), 7.45(\mathrm{~s}, 1 \mathrm{H}), 4.00(\mathrm{~m}, 2 \mathrm{H}), 3.89(\mathrm{~m}, 2 \mathrm{H}), 2.79-2.82(\mathrm{~m}, 2 \mathrm{H}), 2.72\left(\mathrm{td}, J_{1}=12.9 \mathrm{~Hz}\right.$ $\left.J_{2}=4.8 \mathrm{~Hz}, 2 \mathrm{H}\right), 2.34(\mathrm{q}, J=7.2 \mathrm{~Hz}, 2 \mathrm{H}), 2.13(\mathrm{t}, J=10.8 \mathrm{~Hz}, 2 \mathrm{H}), 1.56(\mathrm{~d}, J=11.6 \mathrm{~Hz}, 2$ H), $1.01(\mathrm{t}, J=7.2 \mathrm{~Hz}, 3 \mathrm{H})$.

\section{Preparation of amides $7 \mathbf{a}-7 \mathbf{p}$}

General procedure A:

To a solution of the respective carboxylic acid (commercially available) $(0.1 \mathrm{mmol})$ in $1 \mathrm{~mL}$ DMF was added the respective amine (commercially available) $(0.11$ to $0.15 \mathrm{mmol})$. DIPEA ( $0.3 \mathrm{mmol} ; 0.6 \mathrm{mmol}$ if the amine was a hydrochloride salt) was then added followed by HATU or TBTU (0.11 to $0.15 \mathrm{mmol})$. The reaction mixture was stirred overnight at rt. The crude mixture was directly purified by prep. LC-MS under basic conditions.

4-(2,4-Difluoro-phenyl)-N-(2-dimethylamino-ethyl)-4-oxo-butyramide (7a). General procedure A from 4-(2,4-difluorophenyl)-4-oxobutanoic acid and 2(dimethylamino)ethylamine. QC LC-MS: $t_{R}=0.39 \mathrm{~min} ;[\mathrm{M}+\mathrm{H}]^{+}=285.2$. LC-HRMS: $\mathrm{t}_{\mathrm{R}}=0.36$ 
$\min ; \mathrm{m} / \mathrm{z}=284.1336$, found $=285.1415[\mathrm{M}+\mathrm{H}]^{+} .{ }^{1} \mathrm{H}$ NMR $\left(500 \mathrm{MHz}, \mathrm{D}_{6}-\mathrm{DMSO}\right) \delta: 8.39(\mathrm{~s}$, $1 \mathrm{H}), 7.78\left(\mathrm{td}, J_{1}=6.9 \mathrm{~Hz}, J_{2}=9.1 \mathrm{~Hz}, 1 \mathrm{H}\right), 7.27\left(\mathrm{ddd}, J_{1}=2.5 \mathrm{~Hz}, J_{2}=9.2 \mathrm{~Hz}, J_{3}=11.7 \mathrm{~Hz}\right.$, $1 \mathrm{H}), 7.14\left(\mathrm{td}, J_{1}=2.1 \mathrm{~Hz}, J_{2}=8.3 \mathrm{~Hz}, 1 \mathrm{H}\right), 2.53-2.62(\mathrm{~m}, 1 \mathrm{H}), 2.44-2.49(\mathrm{~m}, 2 \mathrm{H}), 2.32-2.41$ (m, $2 \mathrm{H}), 2.19-2.28(\mathrm{~m}, 1 \mathrm{H}), 2.14-2.17(\mathrm{~m}, 1 \mathrm{H}), 2.13(\mathrm{~s}, 1 \mathrm{H}), 2.03(\mathrm{~s}, 6 \mathrm{H})$.

1-p-Tolyl-1H-[1,2,3]triazole-4-carboxylic acid (2-diethylamino-ethyl)-amide (7b). General procedure A from 1-(4-methylphenyl)-1H-1,2,3-triazole-4-carboxylic acid and 2(diethylamino)ethylamine. QC LC-MS: $t_{R}=0.52 \mathrm{~min} ;[\mathrm{M}+\mathrm{H}]^{+}=302.3$. LC-HRMS: $\mathrm{t}_{\mathrm{R}}=0.46$ $\min ; \mathrm{m} / \mathrm{z}=301.1903$, found $=302.1983[\mathrm{M}+\mathrm{H}]^{+} .{ }^{1} \mathrm{H}$ NMR $\left(500 \mathrm{MHz}, \mathrm{D}_{6}-\mathrm{DMSO}\right) \delta$ : $9.21(\mathrm{~s}$, $1 \mathrm{H}), 8.42(\mathrm{t}, J=5.6 \mathrm{~Hz}, 1 \mathrm{H}), 7.85\left(\mathrm{td}, J_{1}=4.2 \mathrm{~Hz}, J_{2}=2.4 \mathrm{~Hz}, 2 \mathrm{H}\right), 7.40-7.44(\mathrm{~m}, 2 \mathrm{H})$, 3.33-3.39 (m, $2 \mathrm{H}), 2.58(\mathrm{t}, J=7.0 \mathrm{~Hz}, 2 \mathrm{H}), 2.50-2.55(\mathrm{~m}, 4 \mathrm{H}), 2.40-2.43(\mathrm{~m}, 3 \mathrm{H}), 0.98(\mathrm{t}, J$ $=7.1 \mathrm{~Hz}, 6 \mathrm{H})$.

\section{1-(2,4-Difluoro-phenyl)-4-(8-ethyl-1-oxa-4,8-diaza-spiro[4.5]dec-4-yl)-butane-1,4-dione}

(7c). General procedure A from 4-(2,4-difluorophenyl)-4-oxobutanoic acid and 8-ethyl-1-oxa4,8-diazaspiro[4.5]decane. QC LC-MS: $\mathrm{t}_{\mathrm{R}}=0.52 \mathrm{~min} ;[\mathrm{M}+\mathrm{H}]^{+}=367.3$. LC-HRMS: $\mathrm{t}_{\mathrm{R}}=0.46$ $\min ; \mathrm{m} / \mathrm{z}=366.1755$, found $=367.184[\mathrm{M}+\mathrm{H}]^{+} .{ }^{1} \mathrm{H} \mathrm{NMR}\left(500 \mathrm{MHz}, \mathrm{D}_{6}-\mathrm{DMSO}\right) \delta: 7.93(\mathrm{td}$, $\left.J_{1}=6.7 \mathrm{~Hz}, J_{2}=8.5 \mathrm{~Hz}, 1 \mathrm{H}\right), 7.42\left(\mathrm{ddd}, J_{1}=2.4 \mathrm{~Hz}, J_{2}=9.4 \mathrm{~Hz}, J_{3}=11.6 \mathrm{~Hz}, 1 \mathrm{H}\right), 7.24(\mathrm{td}$, $\left.J_{1}=2.3 \mathrm{~Hz}, J_{2}=8.3 \mathrm{~Hz}, 1 \mathrm{H}\right), 3.95(\mathrm{t}, J=6.3 \mathrm{~Hz}, 2 \mathrm{H}), 3.65(\mathrm{t}, J=6.3 \mathrm{~Hz}, 2 \mathrm{H}), 3.10-3.16(\mathrm{~m}$, $2 \mathrm{H}), 2.69-2.74(\mathrm{~m}, 2 \mathrm{H}), 2.67(\mathrm{t}, J=6.2 \mathrm{~Hz}, 2 \mathrm{H}), 2.52-2.58(\mathrm{~m}, 2 \mathrm{H}), 2.28(\mathrm{q}, J=7.2 \mathrm{~Hz}, 2$ H), 2.01-2.09 (m, $2 \mathrm{H}), 1.36(\mathrm{~d}, J=11.8 \mathrm{~Hz}, 2 \mathrm{H}), 0.96(\mathrm{t}, J=7.1 \mathrm{~Hz}, 3 \mathrm{H})$.

\section{(8-Ethyl-1-oxa-4,8-diaza-spiro[4.5]dec-4-yl)-(1-p-tolyl-1H-[1,2,3]triazol-4-yl)-methanone}

(7d). General procedure A from 1-(4-methylphenyl)-1H-1,2,3-triazole-4-carboxylic acid and 8-ethyl-1-oxa-4,8-diazaspiro[4.5]decane. QC LC-MS: $t_{\mathrm{R}}=0.57 \mathrm{~min} ;[\mathrm{M}+\mathrm{H}]^{+}=356.3$. LCHRMS: $\mathrm{t}_{\mathrm{R}}=0.53 \mathrm{~min} ; \mathrm{m} / \mathrm{z}=355.2008$, found $=356.2088[\mathrm{M}+\mathrm{H}]^{+} .{ }^{1} \mathrm{H}$ NMR $\left(500 \mathrm{MHz}, \mathrm{D}_{6}-\right.$ DMSO) $\delta: 9.25(\mathrm{~s}, 1 \mathrm{H}), 7.86(\mathrm{~d}, J=8.5 \mathrm{~Hz}, 2 \mathrm{H}), 7.42(\mathrm{~d}, J=8.1 \mathrm{~Hz}, 2 \mathrm{H}), 4.14(\mathrm{t}, J=6.2 \mathrm{~Hz}$, 
$2 \mathrm{H}), 4.03(\mathrm{t}, J=6.0 \mathrm{~Hz}, 2 \mathrm{H}), 2.75-2.87(\mathrm{~m}, 4 \mathrm{H}), 2.40(\mathrm{~s}, 3 \mathrm{H}), 2.11-2.23(\mathrm{~m}, 2 \mathrm{H}), 2.08$ (s, 2 H), 1.51-1.60 (m, $2 \mathrm{H}), 1.02(\mathrm{t}, J=7.1 \mathrm{~Hz}, 3 \mathrm{H})$.

rac-1-p-Tolyl-1H-[1,2,3]triazole-4-carboxylic acid (4-methyl-morpholin-2-ylmethyl)amide (7e). General procedure A from 1-(4-methylphenyl)-1H-1,2,3-triazole-4-carboxylic acid and (4-methylmorpholin-2-yl)methanamine. LC-MS method $\mathrm{B}: \mathrm{t}_{\mathrm{R}}=0.73 \mathrm{~min} ;[\mathrm{M}+\mathrm{H}]^{+}$ $=316.3$. LC-HRMS: $\mathrm{t}_{\mathrm{R}}=0.45 \mathrm{~min} ; \mathrm{m} / \mathrm{z}=315.1695$, found $=316.1771[\mathrm{M}+\mathrm{H}]^{+} .{ }^{1} \mathrm{H} \mathrm{NMR}$ (500 MHz, D6-DMSO) $\delta: 9.23(\mathrm{~m}, 1 \mathrm{H}), 8.55$ (t, $J=6.0 \mathrm{~Hz}, 1 \mathrm{H}), 7.82-7.88(\mathrm{~m}, 2 \mathrm{H}), 7.39-$ $7.45(\mathrm{~m}, 2 \mathrm{H}), 3.77-3.82(\mathrm{~m}, 1 \mathrm{H}), 3.60-3.66(\mathrm{~m}, 1 \mathrm{H}), 3.49\left(\mathrm{td}, J_{1}=2.4 \mathrm{~Hz}, J_{2}=11.2 \mathrm{~Hz}, 1 \mathrm{H}\right)$, 3.29-3.40 (m, 1H), 2.68-2.72 (m, $1 \mathrm{H}), 2.53-2.60(\mathrm{~m}, 2 \mathrm{H}), 2.40(\mathrm{~s}, 3 \mathrm{H}), 2.17$ (s, $3 \mathrm{H}), 1.97$ (td, $\left.J_{1}=3.3 \mathrm{~Hz}, J_{2}=11.3 \mathrm{~Hz}, 1 \mathrm{H}\right), 1.74(\mathrm{t}, J=9.9 \mathrm{~Hz}, 1 \mathrm{H})$.

1-p-Tolyl-1H-[1,2,3]triazole-4-carboxylic acid diethylcarbamoylmethyl-amide (7f). General procedure A from 1-(4-methylphenyl)-1H-1,2,3-triazole-4-carboxylic acid and 2amino-N,N-diethyl acetamide hydrochloride. QC LC-MS: $\mathrm{t}_{\mathrm{R}}=0.92 \mathrm{~min} ;[\mathrm{M}+\mathrm{H}]^{+}=316.3$. LCHRMS: $\mathrm{t}_{\mathrm{R}}=0.86 \mathrm{~min} ; \mathrm{m} / \mathrm{z}=315.1695$, found $=316.1772[\mathrm{M}+\mathrm{H}]^{+} .{ }^{1} \mathrm{H}$ NMR $\left(500 \mathrm{MHz}, \mathrm{D}_{6^{-}}\right.$ DMSO) $\delta: 9.28(\mathrm{~s}, 1 \mathrm{H}), 8.41(\mathrm{t}, J=5.3 \mathrm{~Hz}, 1 \mathrm{H}), 7.84-7.90(\mathrm{~m}, 2 \mathrm{H}), 7.42(\mathrm{~d}, J=8.1 \mathrm{~Hz}, 2$ H), 4.11-4.17 (m, 2 H), 3.30-3.37 (m, 4 H), 2.40 (s, $3 \mathrm{H}), 1.17$ (t, $J=7.1 \mathrm{~Hz}, 3 \mathrm{H}), 1.00-1.07$ (m, $3 \mathrm{H})$.

Rac-1-p-Tolyl-1H-[1,2,3]triazole-4-carboxylic acid (1-ethyl-pyrrolidin-2-ylmethyl)amide (7g). General procedure A from 1-(4-methylphenyl)-1H-1,2,3-triazole-4-carboxylic acid and 2-(aminomethyl)-1-ethylpyrrolidine. QC LC-MS: $\mathrm{t}_{\mathrm{R}}=0.53 \mathrm{~min} ;[\mathrm{M}+\mathrm{H}]^{+}=314.3$. LCHRMS: $t_{R}=0.48 \mathrm{~min} ; \mathrm{m} / \mathrm{z}=313.1903$, found $=314.1989[\mathrm{M}+\mathrm{H}]^{+} .{ }^{1} \mathrm{H}$ NMR $(400 \mathrm{MHz}$, DMSO, water suppression) $\delta: 9.13(\mathrm{~s}, 1 \mathrm{H}), 8.39(\mathrm{t}, J=5.8 \mathrm{~Hz}, 1 \mathrm{H}), 7.79(\mathrm{~d}, J=8.3 \mathrm{~Hz}, 2 \mathrm{H})$, $7.40(\mathrm{~d}, J=8.2 \mathrm{~Hz}, 2 \mathrm{H}), 3.09-3.23(\mathrm{~m}, 1 \mathrm{H}), 2.99-3.07(\mathrm{~m}, 1 \mathrm{H}), 2.76-2.88(\mathrm{~m}, 1 \mathrm{H}), 2.57-2.65$ 
(m, $1 \mathrm{H}), 2.37$ (s, $3 \mathrm{H}), 2.19-2.33(\mathrm{~m}, 1 \mathrm{H}), 2.07-2.18(\mathrm{~m}, 1 \mathrm{H}), 1.71-1.84(\mathrm{~m}, 1 \mathrm{H}), 1.51-1.69$ (m, $4 \mathrm{H}), 1.04(\mathrm{t}, J=7.1 \mathrm{~Hz}, 3 \mathrm{H})$.

1-p-Tolyl-1H-[1,2,3]triazole-4-carboxylic acid (3-diethylamino-propyl)-amide (7h). General procedure A from 1-(4-methylphenyl)-1H-1,2,3-triazole-4-carboxylic acid and N,Ndiethyl-1,3-propanediamine. QC LC-MS: $t_{R}=0.54 \mathrm{~min} ;[\mathrm{M}+\mathrm{H}]^{+}=316.3 . \mathrm{LC}-\mathrm{HRMS}: \mathrm{t}_{\mathrm{R}}=0.48$ $\min ; \mathrm{m} / \mathrm{z}=315.2059$, found $=316.2143[\mathrm{M}+\mathrm{H}]^{+} .{ }^{1} \mathrm{H}$ NMR $\left(400 \mathrm{MHz}, \mathrm{D}_{6}-\mathrm{DMSO}\right) \delta: 9.15-$ 9.30 (m, $1 \mathrm{H}), 8.78-8.98$ (m, $1 \mathrm{H})$, 7.68-7.93 (m, $2 \mathrm{H})$, 7.36-7.56 (m, $2 \mathrm{H}), 3.30-3.46(\mathrm{~m}, 1 \mathrm{H})$, 2.91-3.18 (m, $6 \mathrm{H}), 2.34-2.43(\mathrm{~m}, 3 \mathrm{H}), 1.79-1.98(\mathrm{~m}, 2 \mathrm{H}), 1.21-1.25$ (m, $1 \mathrm{H}), 1.16$ (t, $J=7.2$ $\mathrm{Hz}, 6 \mathrm{H})$.

1-(4-Diethylamino-piperidin-1-yl)-4-(2,4-difluoro-phenyl)-butane-1,4-dione (7i). General procedure A from 4-(2,4-difluorophenyl)-4-oxobutanoic acid and 4-diethylamino-piperidine. QC LC-MS: $\mathrm{t}_{\mathrm{R}}=0.47 \mathrm{~min} ;[\mathrm{M}+\mathrm{H}]^{+}=353.3$. LC-HRMS: $\mathrm{t}_{\mathrm{R}}=0.43 \mathrm{~min} ; \mathrm{m} / \mathrm{z}=352.1962$, found $=353.2035[\mathrm{M}+\mathrm{H}]^{+} .{ }^{1} \mathrm{H} \mathrm{NMR}(500 \mathrm{MHz}, \mathrm{DMSO}) \delta: 7.92\left(\mathrm{td}, J_{1}=7.0 \mathrm{~Hz}, J_{2}=8.9 \mathrm{~Hz} .1 \mathrm{H}\right)$, $7.42\left(\mathrm{ddd}, J_{1}=2.6 \mathrm{~Hz}, J_{2}=9.3 \mathrm{~Hz}, J_{3}=11.7 \mathrm{~Hz}, 1 \mathrm{H}\right), 7.24\left(\mathrm{td}, J_{1}=2.4 \mathrm{~Hz}, J_{2}=8.4 \mathrm{~Hz}, 1 \mathrm{H}\right)$, 4.32-4.39 (m, $1 \mathrm{H}), 3.92-3.99$ (m, $1 \mathrm{H}), 3.07-3.19$ (m, $2 \mathrm{H}), 2.96-3.04$ (m, $1 \mathrm{H})$, 2.66-2.74 (m, $3 \mathrm{H}), 2.45-2.49$ (m, $4 \mathrm{H}), 1.69-1.75(\mathrm{~m}, 1 \mathrm{H}), 1.61-1.68(\mathrm{~m}, 1 \mathrm{H}), 1.31-1.42(\mathrm{~m}, 1 \mathrm{H}), 1.12-1.23$ (m, $1 \mathrm{H}), 0.96(\mathrm{t}, \mathrm{J}=7.0 \mathrm{~Hz}, 6 \mathrm{H})$.

rac-4-(2,4-Difluoro-phenyl)-N-[2-(1-methyl-pyrrolidin-2-yl)-ethyl]-4-oxo-butyramide

(7j). General procedure A from 4-(2,4-difluorophenyl)-4-oxobutanoic acid and 2-(2aminoethyl)-1-methylpyrrolidine. QC LC-MS: $\mathrm{t}_{\mathrm{R}}=0.47 \mathrm{~min} ;[\mathrm{M}+\mathrm{H}]^{+}=325.3$. LC-HRMS: $\mathrm{t}_{\mathrm{R}}$ $=0.41 \mathrm{~min} ; \mathrm{m} / \mathrm{z}=325.1727$, found $=325.1727[\mathrm{M}+\mathrm{H}]^{+}$.

rac-1-p-Tolyl-1H-[1,2,3]triazole-4-carboxylic acid [2-(1-methyl-pyrrolidin-2-yl)-ethyl]amide (7k). General procedure A from 1-(4-methylphenyl)-1H-1,2,3-triazole-4-carboxylic acid and 2-(2-aminoethyl)-1-methylpyrrolidine. QC LC-MS: $\mathrm{t}_{\mathrm{R}}=0.52 \mathrm{~min} ;[\mathrm{M}+\mathrm{H}]^{+}=314.3$. 
LC-HRMS: $\mathrm{t}_{\mathrm{R}}=0.47 \mathrm{~min} ; \mathrm{m} / \mathrm{z}=313.1903$, found $=314.1982[\mathrm{M}+\mathrm{H}]^{+} .{ }^{1} \mathrm{H} \mathrm{NMR}(500 \mathrm{MHz}$, D6-DMSO) $\delta$ : $9.19(\mathrm{~s}, 1 \mathrm{H}), 8.71(\mathrm{t}, J=5.6 \mathrm{~Hz}, 1 \mathrm{H}), 7.85(\mathrm{~d}, J=8.4 \mathrm{~Hz}, 2 \mathrm{H}), 7.42(\mathrm{~d}, J=8.2$ Hz, 2 H), 2.92-3.02 (m, 1 H), 2.40 (s, 3 H), 2.23 (s, 3 H), 2.03-2.14 (m, 4 H), 1.83-2.00 (m, 2 H), 1.59-1.69 (m, 2 H), 1.40-1.53 (m, 2 H).

4-(2,4-Difluoro-phenyl)-N-(1-methyl-piperidin-4-yl)-4-oxo-butyramide (7l). General procedure A from 4-(2,4-difluorophenyl)-4-oxobutanoic acid and 4-amino-1methylpiperidine. QC LC-MS: $\mathrm{t}_{\mathrm{R}}=0.40 \mathrm{~min} ;[\mathrm{M}+\mathrm{H}]^{+}=311.2 . \mathrm{LC}-\mathrm{HRMS}: \mathrm{t}_{\mathrm{R}}=0.37 \mathrm{~min} ; \mathrm{m} / \mathrm{z}$ $=311.1565$, found $=311.1571[\mathrm{M}+\mathrm{H}]^{+} .{ }^{1} \mathrm{H}$ NMR $\left(500 \mathrm{MHz}, \mathrm{D}_{6}-\mathrm{DMSO}\right) \delta:$ 7.87-7.96 $(\mathrm{m}, 1$ H), $7.80(\mathrm{~d}, J=7.6 \mathrm{~Hz}, 1 \mathrm{H}), 7.42\left(\mathrm{ddd}, J_{1}=2.4 \mathrm{~Hz}, J_{2}=9.3 \mathrm{~Hz}, J_{3}=11.6 \mathrm{~Hz}, 1 \mathrm{H}\right), 7.23(\mathrm{td}$, $\left.J_{1}=2.3 \mathrm{~Hz}, J_{2}=8.3 \mathrm{~Hz}, 1 \mathrm{H}\right), 3.40-3.52(\mathrm{~m}, 1 \mathrm{H}), 3.13\left(\mathrm{td}, J_{1}=2.7 \mathrm{~Hz}, J_{2}=6.6 \mathrm{~Hz}, 2 \mathrm{H}\right), 2.65-$ $2.71(\mathrm{~m}, 2 \mathrm{H}), 2.45(\mathrm{t}, J=6.6 \mathrm{~Hz}, 2 \mathrm{H}), 2.13(\mathrm{~s}, 3 \mathrm{H}), 1.85-1.93(\mathrm{~m}, 2 \mathrm{H}), 1.63-1.70(\mathrm{~m}, 2 \mathrm{H})$, $1.32-1.43(\mathrm{~m}, 2 \mathrm{H})$.

4-(2,4-Difluoro-phenyl)-N-(1-isopropyl-piperidin-4-yl)-4-oxo-butyramide (7m). General procedure A from 4-(2,4-difluorophenyl)-4-oxobutanoic acid and 1-isopropylpiperidin-4amine dihydrochloride. QC LC-MS: $\mathrm{t}_{\mathrm{R}}=0.44 \mathrm{~min} ;[\mathrm{M}+\mathrm{H}]^{+}=339.3 . \mathrm{LC}-\mathrm{HRMS}: \mathrm{t}_{\mathrm{R}}=0.41 \mathrm{~min}$; $\mathrm{m} / \mathrm{z}=338.1806$, found $=339.1886[\mathrm{M}+\mathrm{H}]^{+} .{ }^{1} \mathrm{H}$ NMR $\left(500 \mathrm{MHz}, \mathrm{D}_{6}-\mathrm{DMSO}\right) \delta: 7.92\left(\mathrm{td}, J_{1}\right.$ $\left.=6.9 \mathrm{~Hz}, J_{2}=8.7 \mathrm{~Hz}, 1 \mathrm{H}\right), 7.78(\mathrm{~d}, J=7.7 \mathrm{~Hz}, 1 \mathrm{H}), 7.42\left(\mathrm{ddd}, J_{1}=2.4 \mathrm{~Hz}, J_{2}=9.3 \mathrm{~Hz}, J_{3}=\right.$ $11.6 \mathrm{~Hz}, 1 \mathrm{H}), 7.23\left(\mathrm{td}, J_{1}=2.3 \mathrm{~Hz}, J_{2}=8.3 \mathrm{~Hz}, 1 \mathrm{H}\right), 3.40-3.51(\mathrm{~m}, 1 \mathrm{H}), 3.13\left(\mathrm{td}, J_{1}=2.7\right.$ $\left.\mathrm{Hz}, J_{2}=6.6 \mathrm{~Hz}, 2 \mathrm{H}\right), 2.61-2.75(\mathrm{~m}, 3 \mathrm{H}), 2.45(\mathrm{t}, J=6.6 \mathrm{~Hz}, 2 \mathrm{H}), 2.07-2.15(\mathrm{~m}, 2 \mathrm{H}), 1.64-$ $1.73(\mathrm{~m}, 2 \mathrm{H}), 1.26-1.38(\mathrm{~m}, 2 \mathrm{H}), 0.94(\mathrm{~d}, J=6.6 \mathrm{~Hz}, 6 \mathrm{H})$.

1-p-Tolyl-1H-[1,2,3]triazole-4-carboxylic acid (1-isopropyl-piperidin-4-yl)-amide (7n). General procedure A from 1-(4-methylphenyl)-1H-1,2,3-triazole-4-carboxylic acid and 1isopropylpiperidin-4-amine dihydrochloride. QC LC-MS: $\mathrm{t}_{\mathrm{R}}=0.52 \mathrm{~min} ;[\mathrm{M}+\mathrm{H}]^{+}=328.3$. LCHRMS: $\mathrm{t}_{\mathrm{R}}=0.48 \mathrm{~min} ; \mathrm{m} / \mathrm{z}=327.2059$, found $=328.2135[\mathrm{M}+\mathrm{H}]^{+} .{ }^{1} \mathrm{H}$ NMR $\left(500 \mathrm{MHz}, \mathrm{D}_{6}-\right.$ 
DMSO) $\delta: 9.21(\mathrm{~s}, 1 \mathrm{H}), 8.44(\mathrm{~d}, J=8.1 \mathrm{~Hz}, 1 \mathrm{H}), 7.84(\mathrm{~d}, J=8.4 \mathrm{~Hz}, 2 \mathrm{H}), 7.42(\mathrm{~d}, J=8.2$ $\mathrm{Hz}, 2 \mathrm{H}), 3.71-3.83$ (m, $1 \mathrm{H}), 2.76-2.86$ (m, $2 \mathrm{H}), 2.67-2.76$ (m, $1 \mathrm{H}), 2.40$ (s, 3H), 2.13-2.24 (m, $2 \mathrm{H}), 1.73-1.81(\mathrm{~m}, 2 \mathrm{H}), 1.55-1.68(\mathrm{~m}, 2 \mathrm{H}), 0.98(\mathrm{~d}, J=6.6 \mathrm{~Hz}, 6 \mathrm{H})$.

4-(2,4-Difluoro-phenyl)-N-(1-cyclohexyl-piperidin-4-yl)-4-oxo-butyramide (7o). General procedure A from 4-(2,4-difluorophenyl)-4-oxobutanoic acid and 1-cyclohexylpiperidin-4ylamine. QC LC-MS: $t_{\mathrm{R}}=0.55 \mathrm{~min} ;[\mathrm{M}+\mathrm{H}]^{+}=379.4$. LC-HRMS: $\mathrm{t}_{\mathrm{R}}=0.50 \mathrm{~min} ; \mathrm{m} / \mathrm{z}=$ 378.2119, found $=379.2190[\mathrm{M}+\mathrm{H}]^{+} .{ }^{1} \mathrm{H}$ NMR (500 MHz, D $\left.-\mathrm{DMSO}\right) \delta: 7.92\left(\mathrm{td}, J_{1}=6.8\right.$ $\left.\mathrm{Hz}, J_{2}=8.7 \mathrm{~Hz}, 1 \mathrm{H}\right), 7.77(\mathrm{~d}, J=7.7 \mathrm{~Hz}, 1 \mathrm{H}), 7.42\left(\mathrm{ddd}, J_{1}=2.5 \mathrm{~Hz}, J_{2}=9.4 \mathrm{~Hz}, J_{3}=11.6\right.$ $\mathrm{Hz}, 1 \mathrm{H}), 7.23\left(\mathrm{td}, J_{1}=2.4 \mathrm{~Hz}, J_{2}=8.3 \mathrm{~Hz}, 1 \mathrm{H}\right), 3.39-3.51(\mathrm{~m}, 1 \mathrm{H}), 3.13\left(\mathrm{td}, J_{1}=2.7 \mathrm{~Hz}, J_{2}\right.$ $=6.6 \mathrm{~Hz}, 2 \mathrm{H}), 2.70-2.81(\mathrm{~m}, 2 \mathrm{H}), 2.45(\mathrm{t}, J=6.6 \mathrm{~Hz}, 2 \mathrm{H}), 2.13-2.29(\mathrm{~m}, 3 \mathrm{H}), 1.63-1.80(\mathrm{~m}$, $6 \mathrm{H}), 1.52-1.62(\mathrm{~m}, 1 \mathrm{H}), 1.26-1.39$ (m, $2 \mathrm{H}), 1.12-1.26$ (m, $4 \mathrm{H}), 0.97-1.11(\mathrm{~m}, 1 \mathrm{H})$.

1-p-Tolyl-1H-[1,2,3]triazole-4-carboxylic acid (1-cyclohexyl-piperidin-4-yl)-amide (7p). General procedure A from 1-(4-methylphenyl)-1H-1,2,3-triazole-4-carboxylic acid and 1cyclohexylpiperidin-4-ylamine. QC LC-MS: $\mathrm{t}_{\mathrm{R}}=0.62 \mathrm{~min} ;[\mathrm{M}+\mathrm{H}]^{+}=368.4$. LC-HRMS: $\mathrm{t}_{\mathrm{R}}=$ $0.54 \mathrm{~min} ; \mathrm{m} / \mathrm{z}=367.2372$, found $=368.2445[\mathrm{M}+\mathrm{H}]^{+} .{ }^{1} \mathrm{H}$ NMR $(500 \mathrm{MHz}, \mathrm{D} 6-\mathrm{DMSO}) \delta$ : $9.20(\mathrm{~d}, J=1.5 \mathrm{~Hz}, 1 \mathrm{H}), 8.42(\mathrm{~d}, J=8.2 \mathrm{~Hz}, 1 \mathrm{H}), 7.84(\mathrm{~d}, J=7.0 \mathrm{~Hz}, 2 \mathrm{H}), 7.41(\mathrm{~d}, J=7.3$ Hz, 2 H), 3.69-3.84 (m, 1 H), 2.80-2.87 (m, 2 H), 2.34-2.43 (m, 3 H), 2.17-2.33 (m, 3 H), 1.681.90 (m, $6 \mathrm{H}), 1.49-1.69$ (m, $3 \mathrm{H}), 1.12-1.32(\mathrm{~m}, 4 \mathrm{H}), 1.00-1.13(\mathrm{~m}, 1 \mathrm{H})$.

\section{Preparation of amides 8a-8n}

1-Phenyl-1H-[1,2,3]triazole-4-carboxylic acid (1-cyclohexyl-piperidin-4-yl)-amide (8a).

General procedure A from 1-phenyl-1H-1,2,3-triazole-4-carboxylic acid and 1cyclohexylpiperidin-4-ylamine. QC LC-MS: $\mathrm{t}_{\mathrm{R}}=0.55 \mathrm{~min} ;[\mathrm{M}+\mathrm{H}]^{+}=354.4$. LC-HRMS: $\mathrm{t}_{\mathrm{R}}=$ $0.49 \mathrm{~min} ; \mathrm{m} / \mathrm{z}=353.2216$, found $=354.2286[\mathrm{M}+\mathrm{H}]^{+} .{ }^{1} \mathrm{H}$ NMR $\left(500 \mathrm{MHz}, \mathrm{D}_{6}-\mathrm{DMSO}\right) \delta$ : $9.27(\mathrm{~s}, 1 \mathrm{H}), 8.46(\mathrm{~d}, J=7.9 \mathrm{~Hz}, 1 \mathrm{H}), 7.97$ (d, $J=7.7 \mathrm{~Hz}, 2 \mathrm{H}), 7.59-7.66$ (m, $2 \mathrm{H}), 7.49-7.57$ 
(m, $1 \mathrm{H}), 3.72-3.85$ (m, $1 \mathrm{H}), 2.79-2.92(\mathrm{~m}, 2 \mathrm{H}), 2.24-2.35$ (m, $3 \mathrm{H}), 1.70-1.83$ (m, $6 \mathrm{H}), 1.52-$ 1.69 (m, $3 \mathrm{H}), 1.15-1.28$ (m, $4 \mathrm{H}), 1.03-1.13(\mathrm{~m}, 1 \mathrm{H})$.

5-(4-Bromo-phenyl)-isoxazole-3-carboxylic acid (1-cyclohexyl-piperidin-4-yl)-amide

(8b). General procedure A from 5-(4-bromo-phenyl)isoxazole-3-carboxylic acid and 1cyclohexylpiperidin-4-ylamine. QC LC-MS: $\mathrm{t}_{\mathrm{R}}=0.73 \mathrm{~min} ;[\mathrm{M}+\mathrm{H}]^{+}=432.3$. LC-HRMS: $\mathrm{t}_{\mathrm{R}}=$ $0.64 \mathrm{~min} ; \mathrm{m} / \mathrm{z}=431.1208$, found $=432.1293[\mathrm{M}+\mathrm{H}]^{+} .{ }^{1} \mathrm{H}$ NMR $\left(500 \mathrm{MHz}, \mathrm{D}_{6}-\mathrm{DMSO}\right) \delta$ : $8.69(\mathrm{~d}, J=8.5 \mathrm{~Hz}, 1 \mathrm{H}), 7.87-7.91(\mathrm{~m}, 2 \mathrm{H}), 7.75-7.81(\mathrm{~m}, 2 \mathrm{H}), 7.41(\mathrm{~s}, 1 \mathrm{H}), 3.66-3.79(\mathrm{~m}, 1$ H), 2.78-2.87 (m, 2 H), 2.18-2.31 (m, 3 H), 1.67-1.81 (m, 6 H), 1.50-1.63 (m, 3 H), 1.13-1.28 (m, $4 \mathrm{H}), 1.00-1.12(\mathrm{~m}, 1 \mathrm{H})$.

N-(1-Cyclohexyl-piperidin-4-yl)-4-oxo-4-phenyl-butyramide (8c). General procedure A from 3-benzoylpropionic acid and 1-cyclohexylpiperidin-4-ylamine. QC LC-MS: $t_{R}=0.49$ $\min ;[\mathrm{M}+\mathrm{H}]^{+}=343.4$. LC-HRMS: $\mathrm{t}_{\mathrm{R}}=0.46 \mathrm{~min} ; \mathrm{m} / \mathrm{z}=342.2307$, found $=343.2383[\mathrm{M}+\mathrm{H}]^{+}$.

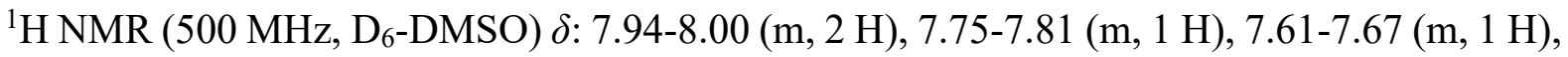
7.50-7.56 (m, 2 H), 3.40-3.51 (m, $1 \mathrm{H}), 3.22(\mathrm{t}, J=6.7 \mathrm{~Hz}, 2 \mathrm{H}), 2.71-2.80(\mathrm{~m}, 2 \mathrm{H}), 2.45(\mathrm{t}, J$ $=6.6 \mathrm{~Hz}, 2 \mathrm{H}), 2.14-2.28(\mathrm{~m}, 3 \mathrm{H}), 1.63-1.78(\mathrm{~m}, 6 \mathrm{H}), 1.53-1.61(\mathrm{~m}, 1 \mathrm{H}), 1.27-1.38(\mathrm{~m}, 2 \mathrm{H})$, 1.12-1.24 (m, $4 \mathrm{H}), 1.00-1.11(\mathrm{~m}, 1 \mathrm{H})$.

N-(1-Cyclohexyl-piperidin-4-yl)-4-(4-fluoro-phenyl)-4-oxo-butyramide (8d). General procedure A from 3-(4-fluorobenzoyl)propionic acid and 1-cyclohexylpiperidin-4-ylamine. QC LC-MS: $\mathrm{t}_{\mathrm{R}}=0.52 \mathrm{~min} ;[\mathrm{M}+\mathrm{H}]^{+}=361.3 . \mathrm{LC}-\mathrm{HRMS}: \mathrm{t}_{\mathrm{R}}=0.48 \mathrm{~min} ; \mathrm{m} / \mathrm{z}=360.2213$, found $=361.229[\mathrm{M}+\mathrm{H}]^{+} .{ }^{1} \mathrm{H}$ NMR (500 MHz, D $\left.-\mathrm{DMSO}\right) \delta: 8.03-8.08(\mathrm{~m}, 2 \mathrm{H}), 7.77(\mathrm{~d}, J=7.7$ $\mathrm{Hz}, 1 \mathrm{H}), 7.32-7.39$ (m, $2 \mathrm{H}), 3.40-3.50(\mathrm{~m}, 1 \mathrm{H}), 3.20(\mathrm{t}, J=6.6 \mathrm{~Hz}, 2 \mathrm{H}), 2.72-2.79(\mathrm{~m}, 2 \mathrm{H})$, $2.45(\mathrm{t}, J=6.6 \mathrm{~Hz}, 2 \mathrm{H}), 2.14-2.28(\mathrm{~m}, 3 \mathrm{H}), 1.65-1.77(\mathrm{~m}, 6 \mathrm{H}), 1.53-1.60(\mathrm{~m}, 1 \mathrm{H}), 1.26-1.37$ (m, 2 H), 1.12-1.24 (m, 4 H), 0.99-1.11 (m, 1 H). 
4-(4-Bromo-phenyl)-N-(1-cyclohexyl-piperidin-4-yl)-4-oxo-butyramide (8e). General

procedure A from 4-(4-bromo-phenyl)-4-oxobutyric acid and 1-cyclohexylpiperidin-4ylamine. QC LC-MS: $\mathrm{t}_{\mathrm{R}}=0.63 \mathrm{~min} ;[\mathrm{M}+\mathrm{H}]^{+}=421.3 . \mathrm{LC}-\mathrm{HRMS}: \mathrm{t}_{\mathrm{R}}=0.56 \mathrm{~min} ; \mathrm{m} / \mathrm{z}=$ 420.1412, found $=421.1495[\mathrm{M}+\mathrm{H}]^{+} .{ }^{1} \mathrm{H}$ NMR (500 MHz, D 6 -DMSO) $\delta: 7.90\left(\mathrm{~m}, J_{1}=4.3\right.$ $\left.\mathrm{Hz}, J_{2}=2.4 \mathrm{~Hz}, 2 \mathrm{H}\right), 7.78(\mathrm{~d}, J=7.7 \mathrm{~Hz}, 1 \mathrm{H}), 7.74\left(\mathrm{~m}, J_{1}=4.3 \mathrm{~Hz}, J_{2}=2.4 \mathrm{~Hz}, 2 \mathrm{H}\right), 3.39-$ $3.50(\mathrm{~m}, 1 \mathrm{H}), 3.19(\mathrm{t}, J=6.6 \mathrm{~Hz}, 2 \mathrm{H}), 2.71-2.80(\mathrm{~m}, 2 \mathrm{H}), 2.45(\mathrm{t}, J=6.7 \mathrm{~Hz}, 2 \mathrm{H}), 2.14-2.28$ $(\mathrm{m}, 3 \mathrm{H}), 1.64-1.77(\mathrm{~m}, 6 \mathrm{H}), 1.52-1.60(\mathrm{~m}, 1 \mathrm{H}), 1.31\left(\mathrm{qd}, J_{1}=3.8 \mathrm{~Hz}, J_{2}=11.6 \mathrm{~Hz}, 2 \mathrm{H}\right), 1.13-$ $1.22(\mathrm{~m}, 4 \mathrm{H}), 0.99-1.11(\mathrm{~m}, 1 \mathrm{H})$.

4-(4-Chloro-2-fluoro-phenyl)-N-(1-cyclohexyl-piperidin-4-yl)-4-oxo-butyramide

(8f).

General procedure A from 4-(4-chloro-2-fluorophenyl)-4-oxobutyric acid and 1cyclohexylpiperidin-4-ylamine. QC LC-MS: $t_{\mathrm{R}}=0.58 \mathrm{~min} ;[\mathrm{M}+\mathrm{H}]^{+}=395.2$. LC-HRMS: $\mathrm{t}_{\mathrm{R}}=$ $0.56 \mathrm{~min} ; \mathrm{m} / \mathrm{z}=394.1823$, found $=395.1906[\mathrm{M}+\mathrm{H}]^{+} .{ }^{1} \mathrm{H}$ NMR $\left(500 \mathrm{MHz}, \mathrm{D}_{6}-\mathrm{DMSO}\right) \delta$ : $7.84(\mathrm{t}, J=8.3 \mathrm{~Hz}, 1 \mathrm{H}), 7.78(\mathrm{~d}, J=7.7 \mathrm{~Hz}, 1 \mathrm{H}), 7.62\left(\mathrm{dd}, J_{1}=2.0 \mathrm{~Hz}, J_{2}=11.0 \mathrm{~Hz}, 1 \mathrm{H}\right)$, $7.44\left(\mathrm{dd}, J_{1}=2.0 \mathrm{~Hz}, J_{2}=8.5 \mathrm{~Hz}, 1 \mathrm{H}\right), 3.39-3.49(\mathrm{~m}, 1 \mathrm{H}), 3.13\left(\mathrm{td}, J_{1}=2.6 \mathrm{~Hz}, J_{2}=6.6 \mathrm{~Hz}\right.$ $2 \mathrm{H}), 2.72-2.78(\mathrm{~m}, 2 \mathrm{H}), 2.45(\mathrm{t}, J=6.6 \mathrm{~Hz}, 2 \mathrm{H}), 2.15-2.26(\mathrm{~m}, 3 \mathrm{H}), 1.64-1.75(\mathrm{~m}, 6 \mathrm{H})$, 1.52-1.60 (m, $1 \mathrm{H}), 1.26-1.36$ (m, $2 \mathrm{H}), 1.13-1.23$ (m, $4 \mathrm{H}), 1.01-1.10$ (m, $1 \mathrm{H})$.

\section{5-(2,4-Difluoro-phenyl)-isoxazole-3-carboxylic acid (1-cyclohexyl-piperidin-4-yl)-amide}

(8g). General procedure A from 5-(2,4-difluoro-phenyl)-isoxazole-3-carboxylic acid and 1cyclohexylpiperidin-4-ylamine. $\mathrm{LC}-\mathrm{MS}$ method B: $\mathrm{t}_{\mathrm{R}}=1.17 \mathrm{~min} ;[\mathrm{M}+\mathrm{H}]^{+}=390.4$. LC-HRMS: $\mathrm{t}_{\mathrm{R}}=0.58 \mathrm{~min} ; \mathrm{m} / \mathrm{z}=389.1915$, found $=390.1993[\mathrm{M}+\mathrm{H}]^{+} .{ }^{1} \mathrm{H} \mathrm{NMR}\left(500 \mathrm{MHz}, \mathrm{D}_{6}-\mathrm{DMSO}\right)$ $\delta: 8.74(\mathrm{~d}, J=7.9 \mathrm{~Hz}, 1 \mathrm{H}), 8.06\left(\mathrm{td}, J_{1}=6.4 \mathrm{~Hz}, J_{2}=8.7 \mathrm{~Hz}, 1 \mathrm{H}\right), 7.59\left(\mathrm{ddd}, J_{1}=11.6 \mathrm{~Hz}, J_{2}\right.$ $\left.=9.5 \mathrm{~Hz}, J_{3}=2.6 \mathrm{~Hz}, 1 \mathrm{H}\right), 7.34\left(\mathrm{td}, J_{1}=8.3 \mathrm{~Hz}, J_{2}=2.1 \mathrm{~Hz}, 1 \mathrm{H}\right), 7.16(\mathrm{~d}, J=2.9 \mathrm{~Hz}, 1 \mathrm{H})$, 3.67-3.81 (m, $1 \mathrm{H}), 2.78-2.88(\mathrm{~m}, 2 \mathrm{H}), 2.19-2.34$ (m, $3 \mathrm{H}), 1.65-1.84$ (m, $6 \mathrm{H}), 1.46-1.65$ (m, $3 \mathrm{H}), 1.13-1.28$ (m, $4 \mathrm{H}), 1.00-1.13$ (m, $1 \mathrm{H})$. 
5-(2-Fluoro-phenyl)-isoxazole-3-carboxylic acid (1-cyclohexyl-piperidin-4-yl)-amide

(8h). General procedure A from 5-(2-fluoro-phenyl)-isoxazole-3-carboxylic acid and 1cyclohexylpiperidin-4-ylamine. QC LC-MS: $\mathrm{t}_{\mathrm{R}}=0.66 \mathrm{~min} ;[\mathrm{M}+\mathrm{H}]^{+}=372.3$. LC-HRMS: $\mathrm{t}_{\mathrm{R}}=$ $0.57 \mathrm{~min} ; \mathrm{m} / \mathrm{z}=371.2009$, found $=372.2082[\mathrm{M}+\mathrm{H}]^{+} .{ }^{1} \mathrm{H}$ NMR $\left(500 \mathrm{MHz}, \mathrm{D}_{6}-\mathrm{DMSO}\right) \delta$ : $8.73(\mathrm{~d}, J=8.0 \mathrm{~Hz}, 1 \mathrm{H}), 7.99\left(\mathrm{td}, J_{1}=1.6 \mathrm{~Hz}, J_{2}=7.7 \mathrm{~Hz}, 1 \mathrm{H}\right), 7.60-7.68(\mathrm{~m}, 1 \mathrm{H}), 7.40-7.53$ (m, $2 \mathrm{H}), 7.17(\mathrm{~d}, J=3.0 \mathrm{~Hz}, 1 \mathrm{H}), 3.68-3.80(\mathrm{~m}, 1 \mathrm{H}), 2.79-2.87(\mathrm{~m}, 2 \mathrm{H}), 2.20-2.31(\mathrm{~m}, 3 \mathrm{H})$, 1.68-1.82 (m, $6 \mathrm{H}), 1.51-1.63$ (m, $3 \mathrm{H}), 1.13-1.28$ (m, $4 \mathrm{H}), 1.01-1.13$ (m, $1 \mathrm{H})$.

5-(4-Fluoro-phenyl)-isoxazole-3-carboxylic acid (1-cyclohexyl-piperidin-4-yl)-amide (8i).

General procedure A from 5-(4-fluoro-phenyl)-isoxazole-3-carboxylic acid and 1cyclohexylpiperidin-4-ylamine. QC LC-MS: $\mathrm{t}_{\mathrm{R}}=0.66 \mathrm{~min} ;[\mathrm{M}+\mathrm{H}]^{+}=372.4$. LC-HRMS: $\mathrm{t}_{\mathrm{R}}=$ $0.58 \mathrm{~min} ; \mathrm{m} / \mathrm{z}=371.2009$, found $=372.2092[\mathrm{M}+\mathrm{H}]^{+} .{ }^{1} \mathrm{H}$ NMR $\left(500 \mathrm{MHz}, \mathrm{D}_{6}-\mathrm{DMSO}\right) \delta$ : $8.68(\mathrm{~d}, J=8.1 \mathrm{~Hz}, 1 \mathrm{H}), 7.97-8.03(\mathrm{~m}, 2 \mathrm{H}), 7.41(\mathrm{~m}, 2 \mathrm{H}), 7.35(\mathrm{~s}, 1 \mathrm{H}), 3.64-3.81(\mathrm{~m}, 1 \mathrm{H})$, 2.76-2.89 (m, 2 H), 2.17-2.30 (m, 3 H), 1.67-1.84 (m, 6 H), 1.50-1.64 (m, $3 \mathrm{H}), 1.13-1.32$ (m, $4 \mathrm{H}), 0.98-1.12(\mathrm{~m}, 1 \mathrm{H})$.

5-Pyridin-3-yl-isoxazole-3-carboxylic acid (1-cyclohexyl-piperidin-4-yl)-amide (8j). General procedure A from 5-pyridin-3-yl-isoxazole-3-carboxylic acid and 1cyclohexylpiperidin-4-ylamine. QC LC-MS: $\mathrm{t}_{\mathrm{R}}=0.48 \mathrm{~min} ;[\mathrm{M}+\mathrm{H}]^{+}=355.3 . \mathrm{LC}-\mathrm{HRMS}: \mathrm{t}_{\mathrm{R}}=$ $0.42 \mathrm{~min} ; \mathrm{m} / \mathrm{z}=354.2056$, found $=355.2125[\mathrm{M}+\mathrm{H}]^{+} .{ }^{1} \mathrm{H}$ NMR $\left(500 \mathrm{MHz}, \mathrm{D}_{6}-\mathrm{DMSO}\right) \delta$ : 9.14-9.16 (m, $1 \mathrm{H}), 8.70-8.75(\mathrm{~m}, 2 \mathrm{H}), 8.30-8.35(\mathrm{~m}, 1 \mathrm{H}), 7.60\left(\mathrm{dd}, J_{1}=4.8 \mathrm{~Hz}, J_{2}=8.0 \mathrm{~Hz}\right.$, $1 \mathrm{H}), 7.51(\mathrm{~s}, 1 \mathrm{H}), 3.67-3.80(\mathrm{~m}, 1 \mathrm{H}), 2.83(\mathrm{~d}, J=11.4 \mathrm{~Hz}, 2 \mathrm{H}), 2.25(\mathrm{t}, J=11.1 \mathrm{~Hz}, 3 \mathrm{H})$, 1.66-1.81 (m, $6 \mathrm{H}), 1.49-1.64$ (m, $3 \mathrm{H}), 1.14-1.27$ (m, $4 \mathrm{H}), 1.00-1.12(\mathrm{~m}, 1 \mathrm{H})$. 


\section{Preparation of amides $9 \mathrm{a}-9 \mathrm{~g}$}

5-(2,4-Difluoro-phenyl)-isoxazole-3-carboxylic acid (1-acetyl-piperidin-4-yl)-amide (9a).

General procedure A from 5-(2,4-difluoro-phenyl)-isoxazole-3-carboxylic acid and 1-acetyl 4amino-piperidine. QC LC-MS: $\mathrm{t}_{\mathrm{R}}=0.88 \mathrm{~min} ;[\mathrm{M}+\mathrm{H}]^{+}=350.3 . \mathrm{LC}-\mathrm{HRMS}: \mathrm{t}_{\mathrm{R}}=0.83 \mathrm{~min} ; \mathrm{m} / \mathrm{z}$ $=349.1238$, found $=350.1317[\mathrm{M}+\mathrm{H}]^{+} .{ }^{1} \mathrm{H}$ NMR $\left(400 \mathrm{MHz}, \mathrm{D}_{6}-\mathrm{DMSO}\right) \delta: 8.81-8.87(\mathrm{~m}, 1$ H), 8.01-8.09 (m, $1 \mathrm{H})$, 7.54-7.61 (m, $1 \mathrm{H})$, 7.29-7.37 (m, $1 \mathrm{H})$, 7.15-7.18 (m, $1 \mathrm{H})$, 4.31-4.40 (m, $1 \mathrm{H}), 3.98-4.10(\mathrm{~m}, 1 \mathrm{H}), 3.78-3.88(\mathrm{~m}, 1 \mathrm{H}), 3.08-3.18(\mathrm{~m}, 1 \mathrm{H}), 2.61-2.71(\mathrm{~m}, 1 \mathrm{H}), 2.01$ (s, 3 H), 1.73-1.89 (m, 2 H), 1.35-1.58 (m, 2 H).

\section{5-(2,4-Difluoro-phenyl)-isoxazole-3-carboxylic acid piperidin-4-yl-amide hydrochloride}

(9b). General procedure A from 5-(2,4-difluoro-phenyl)-isoxazole-3-carboxylic acid and 1-NBoc-(4-amino)-piperidine, followed by N-Boc group deprotection with $\mathrm{HCl} 2 \mathrm{M}$ in dioxane/DCM 1/1. QC LC-MS: $\mathrm{t}_{\mathrm{R}}=0.54 \mathrm{~min} ;[\mathrm{M}+\mathrm{H}]^{+}=308.2 . \mathrm{LC}-\mathrm{HRMS}: \mathrm{t}_{\mathrm{R}}=0.48 \mathrm{~min} ; \mathrm{m} / \mathrm{z}$ $=307.1132$, found $=308.1213[\mathrm{M}+\mathrm{H}]^{+} .{ }^{1} \mathrm{H}$ NMR $\left(500 \mathrm{MHz}, \mathrm{D}_{6}-\mathrm{DMSO}\right) \delta: 9.06(\mathrm{~d}, J=7.6$ $\mathrm{Hz}, 2 \mathrm{H}), 8.94-8.94(\mathrm{~m}, 1 \mathrm{H}), 8.06\left(\mathrm{td}, J_{1}=8.7 \mathrm{~Hz}, J_{2}=6.5 \mathrm{~Hz}, 1 \mathrm{H}\right), 7.59\left(\mathrm{ddd}, J_{1}=11.5 \mathrm{~Hz}\right.$, $\left.J_{2}=9.4 \mathrm{~Hz}, J_{3}=2.4 \mathrm{~Hz}, 1 \mathrm{H}\right), 7.34\left(\mathrm{td}, J_{1}=8.3 \mathrm{~Hz}, J_{2}=2.1 \mathrm{~Hz}, 1 \mathrm{H}\right), 7.22(\mathrm{~d}, J=2.9 \mathrm{~Hz}, 1$ H), 4.05-4.16 (m, $1 \mathrm{H}), 3.30(\mathrm{~d}, J=12.7 \mathrm{~Hz}, 2 \mathrm{H}), 2.95-3.05(\mathrm{~m}, 2 \mathrm{H}), 1.96-2.03(\mathrm{~m}, 2 \mathrm{H})$, $1.77-1.90(\mathrm{~m}, 2 \mathrm{H})$.

\section{5-(2,4-Difluoro-phenyl)-isoxazole-3-carboxylic acid (1-cyclopentyl-piperidin-4-yl)-amide}

(9c). General procedure A from 5-(2,4-difluoro-phenyl)-isoxazole-3-carboxylic acid and 1cyclopentylpiperidin-4-ylamine. QC LC-MS: $\mathrm{t}_{\mathrm{R}}=0.62 \mathrm{~min} ;[\mathrm{M}+\mathrm{H}]^{+}=376.4$. LC-HRMS: $\mathrm{t}_{\mathrm{R}}=$ $0.56 \mathrm{~min} ; \mathrm{m} / \mathrm{z}=375.1758$, found $=376.1837[\mathrm{M}+\mathrm{H}]^{+} .{ }^{1} \mathrm{H}$ NMR $\left(500 \mathrm{MHz}, \mathrm{D}_{6}-\mathrm{DMSO}\right) \delta$ : $8.74(\mathrm{~d}, J=7.9 \mathrm{~Hz}, 1 \mathrm{H}), 8.06\left(\mathrm{td}, J_{1}=6.5 \mathrm{~Hz}, J_{2}=8.6 \mathrm{~Hz}, 1 \mathrm{H}\right), 7.58\left(\mathrm{ddd}, J_{1}=2.5 \mathrm{~Hz}, J_{2}=\right.$ $\left.9.3 \mathrm{~Hz}, J_{3}=11.4 \mathrm{~Hz}, 1 \mathrm{H}\right), 7.33\left(\mathrm{td}, J_{1}=2.6 \mathrm{~Hz}, J_{2}=8.7 \mathrm{~Hz}, 1 \mathrm{H}\right), 7.15(\mathrm{~d}, J=2.9 \mathrm{~Hz}, 1 \mathrm{H})$, 3.70-3.84 (m, $1 \mathrm{H}), 2.89-3.03(\mathrm{~m}, 2 \mathrm{H}), 1.90-2.05$ (m, $2 \mathrm{H}), 1.71-1.88$ (m, $4 \mathrm{H}), 1.45-1.71$ (m, $6 \mathrm{H}), 1.18-1.42(\mathrm{~m}, 3 \mathrm{H})$. 
5-(2,4-Difluoro-phenyl)-isoxazole-3-carboxylic acid (1-cyclobutyl-piperidin-4-yl)-amide

(9d). General procedure A from 5-(2,4-difluoro-phenyl)-isoxazole-3-carboxylic acid and 1cyclobutylpiperidin-4-ylamine. QC LC-MS: $\mathrm{t}_{\mathrm{R}}=0.59 \mathrm{~min} ;[\mathrm{M}+\mathrm{H}]^{+}=362.3$. LC-HRMS: $\mathrm{t}_{\mathrm{R}}=$ $0.53 \mathrm{~min} ; \mathrm{m} / \mathrm{z}=361.1602$, found $=362.1687[\mathrm{M}+\mathrm{H}]^{+} .{ }^{1} \mathrm{H}$ NMR $\left(500 \mathrm{MHz}, \mathrm{D}_{6}-\mathrm{DMSO}\right) \delta$ : $8.76(\mathrm{~d}, J=8.0 \mathrm{~Hz}, 1 \mathrm{H}), 8.06\left(\mathrm{td}, J_{1}=6.4 \mathrm{~Hz}, J_{2}=8.7 \mathrm{~Hz}, 1 \mathrm{H}\right), 7.58\left(\mathrm{ddd}, J_{1}=2.5 \mathrm{~Hz}, J_{2}=\right.$ $\left.9.3 \mathrm{~Hz}, J_{3}=11.5 \mathrm{~Hz}, 1 \mathrm{H}\right), 7.29-7.37(\mathrm{~m}, 1 \mathrm{H}), 7.16(\mathrm{~d}, J=3.0 \mathrm{~Hz}, 1 \mathrm{H}), 3.69-3.81(\mathrm{~m}, 1 \mathrm{H})$, 2.77-2.83 (m, 2 H), 2.64-2.71 (m, $1 \mathrm{H}), 1.92-2.00$ (m, $2 \mathrm{H}), 1.72-1.82(\mathrm{~m}, 6 \mathrm{H}), 1.53-1.65$ (m, $4 \mathrm{H})$.

5-(2,4-Difluoro-phenyl)-isoxazole-3-carboxylic acid [1-(2,2-difluoro-ethyl)-piperidin-4yl]-amide (9e). General procedure A from 5-(2,4-difluoro-phenyl)-isoxazole-3-carboxylic acid and 1-(2,2-difluoro-ethyl)-piperidin-4-ylamine. LC-MS method $\mathrm{B}: \mathrm{t}_{\mathrm{R}}=0.95 \mathrm{~min} ;[\mathrm{M}+\mathrm{H}]^{+}$ =372.2. LC-HRMS: $\mathrm{t}_{\mathrm{R}}=0.57 \mathrm{~min} ; \mathrm{m} / \mathrm{z}=371.1257$, found $=372.1337[\mathrm{M}+\mathrm{H}]^{+} .{ }^{1} \mathrm{H} \mathrm{NMR}$ $\left(500 \mathrm{MHz}, \mathrm{D}_{6}-\mathrm{DMSO}\right) \delta: 8.77(\mathrm{~d}, J=8.0 \mathrm{~Hz}, 1 \mathrm{H}), 8.06\left(\mathrm{td}, J_{1}=8.7 \mathrm{~Hz}, J 2=6.4 \mathrm{~Hz}, 1 \mathrm{H}\right)$, $7.59\left(\mathrm{ddd}, J_{1}=11.6 \mathrm{~Hz}, J_{2}=9.2 \mathrm{~Hz}, J_{3}=2.6 \mathrm{~Hz}, 1 \mathrm{H}\right), 7.34\left(\mathrm{td}, J_{1}=8.5 \mathrm{~Hz}, J_{2}=2.4 \mathrm{~Hz}, 1 \mathrm{H}\right)$, $7.16(\mathrm{~d}, J=2.9 \mathrm{~Hz}, 1 \mathrm{H}), 6.01-6.25(\mathrm{~m}, 1 \mathrm{H}), 3.73-3.83(\mathrm{~m}, 1 \mathrm{H}), 2.89-2.95(\mathrm{~m}, 2 \mathrm{H}), 2.72(\mathrm{td}$, $\left.J_{1}=15.6 \mathrm{~Hz}, J_{2}=4.3 \mathrm{~Hz}, 2 \mathrm{H}\right), 2.25\left(\mathrm{td}, J_{1}=11.7 \mathrm{~Hz}, J_{2}=2.0 \mathrm{~Hz}, 2 \mathrm{H}\right), 1.72-1.79(\mathrm{~m}, 2 \mathrm{H})$, 1.59-1.68 (m, $2 \mathrm{H})$.

5-(2,4-Difluoro-phenyl)-isoxazole-3-carboxylic acid [1-(2-methoxy-ethyl)-piperidin-4-yl]amide (9f). General procedure A from 5-(2,4-difluoro-phenyl)-isoxazole-3-carboxylic acid and 1-(2-methoxy-ethyl)-piperidin-4-ylamine. QC LC-MS: $\mathrm{t}_{\mathrm{R}}=0.58 \mathrm{~min} ;[\mathrm{M}+\mathrm{H}]^{+}=366.2$. LCHRMS: $\mathrm{t}_{\mathrm{R}}=0.51 \mathrm{~min} ; \mathrm{m} / \mathrm{z}=365.1551$, found $=366.1633[\mathrm{M}+\mathrm{H}]^{+} .{ }^{1} \mathrm{H}$ NMR $\left(500 \mathrm{MHz}, \mathrm{D}_{6^{-}}\right.$ DMSO) $\delta: 8.75(\mathrm{~d}, J=8.0 \mathrm{~Hz}, 1 \mathrm{H}), 8.06\left(\mathrm{td}, J_{1}=6.4 \mathrm{~Hz}, J_{2}=8.7 \mathrm{~Hz}, 1 \mathrm{H}\right), 7.58\left(\mathrm{ddd}, J_{1}=\right.$ $\left.11.5 \mathrm{~Hz}, J_{2}=9.3 \mathrm{~Hz}, J_{3}=2.5 \mathrm{~Hz}, 1 \mathrm{H}\right), 7.29-7.37(\mathrm{~m}, 1 \mathrm{H}), 7.16(\mathrm{~d}, J=2.9 \mathrm{~Hz}, 1 \mathrm{H}), 3.70-3.81$ (m, $1 \mathrm{H}), 3.42(\mathrm{t}, J=5.9 \mathrm{~Hz}, 2 \mathrm{H}), 3.24(\mathrm{~s}, 3 \mathrm{H}), 2.84-2.92(\mathrm{~m}, 2 \mathrm{H}), 2.46(\mathrm{t}, J=5.9 \mathrm{~Hz}, 2 \mathrm{H})$, 1.98-2.07 (m, $2 \mathrm{H}), 1.70-1.79$ (m, $2 \mathrm{H}), 1.55-1.66$ (m, 2 H). 
5-(2,4-Difluoro-phenyl)-isoxazole-3-carboxylic acid (1-benzyl-piperidin-4-yl)-amide (9g).

General procedure A from 5-(2,4-difluoro-phenyl)-isoxazole-3-carboxylic acid and benzylamine. QC LC-MS: $t_{\mathrm{R}}=0.67 \mathrm{~min} ;[\mathrm{M}+\mathrm{H}]^{+}=398.3$. LC-HRMS: $\mathrm{t}_{\mathrm{R}}=0.59 \mathrm{~min} ; \mathrm{m} / \mathrm{z}=$ 397.1602, found $=398.1684[\mathrm{M}+\mathrm{H}]^{+} .{ }^{1} \mathrm{H}$ NMR (500 MHz, D $\left.-\mathrm{DMSO}\right) \delta: 8.76(\mathrm{~d}, J=8.0 \mathrm{~Hz}$, $1 \mathrm{H}), 8.06\left(\mathrm{td}, J_{1}=6.4 \mathrm{~Hz}, J_{2}=8.7 \mathrm{~Hz}, 1 \mathrm{H}\right), 7.58\left(\mathrm{ddd}, J_{1}=2.5 \mathrm{~Hz}, J_{2}=9.3 \mathrm{~Hz}, J_{3}=11.6 \mathrm{~Hz}\right.$, $1 \mathrm{H}), 7.23-7.36(\mathrm{~m}, 6 \mathrm{H}), 7.16(\mathrm{~d}, J=2.9 \mathrm{~Hz}, 1 \mathrm{H}), 3.73-3.85(\mathrm{~m}, 1 \mathrm{H}), 3.47(\mathrm{~s}, 2 \mathrm{H}), 2.77-2.88$ (m, 2 H), 1.95-2.08 (m, 2 H), 1.72-1.82 (m, 2 H), 1.55-1.69 (m, 2 H).

\section{Preparation of amides 10a-10f}

1-(2,4-Difluoro-phenyl)-1H-pyrazole-4-carboxylic acid (1-cyclohexyl-piperidin-4-yl)amide (10a). General procedure A from 1-(2,4-difluorophenyl)-1H-pyrazole-3-carboxylic acid and 1-cyclohexylpiperidin-4-ylamine. QC LC-MS: $\mathrm{t}_{\mathrm{R}}=0.58 \mathrm{~min} ;[\mathrm{M}+\mathrm{H}]^{+}=389.4$. LC-HRMS: $\mathrm{t}_{\mathrm{R}}=0.50 \mathrm{~min} ; \mathrm{m} / \mathrm{z}=388.2075$, found $=389.2147[\mathrm{M}+\mathrm{H}]^{+} .{ }^{1} \mathrm{H} \mathrm{NMR}\left(500 \mathrm{MHz}, \mathrm{D}_{6}-\mathrm{DMSO}\right)$ $\delta: 8.63(\mathrm{~d}, J=2.0 \mathrm{~Hz}, 1 \mathrm{H}), 8.16(\mathrm{~s}, 1 \mathrm{H}), 8.02(\mathrm{~d}, J=7.8 \mathrm{~Hz}, 1 \mathrm{H}), 7.86\left(\mathrm{td}, J_{1}=6.0 \mathrm{~Hz}, J_{2}=\right.$ $9.0 \mathrm{~Hz}, 1 \mathrm{H}), 7.61\left(\mathrm{ddd}, J_{1}=2.7 \mathrm{~Hz}, J_{2}=9.0 \mathrm{~Hz}, J_{3}=11.6 \mathrm{~Hz}, 1 \mathrm{H}\right), 7.26-7.32(\mathrm{~m}, 1 \mathrm{H}), 3.64-$ $3.74(\mathrm{~m}, 1 \mathrm{H}), 2.79-2.87$ (m, $2 \mathrm{H}), 2.19-2.32$ (m, $3 \mathrm{H}), 1.68-1.83(\mathrm{~m}, 6 \mathrm{H}), 1.54-1.62(\mathrm{~m}, 1 \mathrm{H})$, $1.41-1.52(\mathrm{~m}, 2 \mathrm{H}), 1.14-1.27$ (m, $4 \mathrm{H}), 1.01-1.13(\mathrm{~m}, 1 \mathrm{H})$

1-(2,4-Difluoro-phenyl)-1H-[1,2,3]triazole-4-carboxylic acid (1-cyclohexyl-piperidin-4yl)-amide (10b). a) To a solution of ethyl 2-diazo-3-oxopropanoate ${ }^{2}$ (1.6 g, $\left.8.85 \mathrm{mmol}\right)$ in EtOH (3.15 mL) was added glacial acetic acid $(1.27 \mathrm{~mL}, 22.1 \mathrm{mmol})$ followed by 2,4difluoroaniline $(1.22 \mathrm{~g}, 9.47 \mathrm{mmol})$. After stirring overnight, the reaction mixture was concentrated, and the residue was diluted with cold water $(40 \mathrm{~mL})$. The precipitate was filtered, washed with cold water $(10 \mathrm{~mL})$ and dried under HV to afford 1-(2,4-difluoro-phenyl)-1H$[1,2,3]$ triazole-4-carboxylic acid ethyl ester as a beige solid (2.03 g, 91\%). LC-MS method A: $\mathrm{t}_{\mathrm{R}}=0.8 \mathrm{~min} ;[\mathrm{M}+\mathrm{H}]^{+}=254.12$. 
b) To a solution of the above ester $(1.93 \mathrm{~g}, 7.62 \mathrm{mmol})$ in THF $(16 \mathrm{~mL})$ was added $\mathrm{LiOH}$ hydrate $(11.4 \mathrm{mmol})$ dissolved in water $(16 \mathrm{~mL})$. After stirring for $45 \mathrm{~min}$, THF was evaporated and the aqueous residue was cooled to $0^{\circ} \mathrm{C}$. A $1 \mathrm{M} \mathrm{HCl}$ solution was added until $\mathrm{pH}=2$. The precipitated product was filtered, washed with water $(15 \mathrm{~mL})$, and dried under HV. 1-(2,4difluoro-phenyl)-1H-[1,2,3]triazole-4-carboxylic acid was obtained as a beige powder (1.71 g, 100\%). LC-MS method A: $t_{R}=0.61 \min ;[\mathrm{M}+\mathrm{H}]^{+}=225.96,[\mathrm{M}+\mathrm{H}+\mathrm{MeCN}]^{+}=267.10$.

c) General procedure A from the above carboxylic acid and 1-cyclohexylpiperidin-4-ylamine to give 10b. QC LC-MS: $t_{R}=0.56 \mathrm{~min} ;[\mathrm{M}+\mathrm{H}]^{+}=390.3 ;$ LC-HRMS: $\mathrm{t}_{\mathrm{R}}=0.50 \mathrm{~min} ; \mathrm{m} / \mathrm{z}=$ 389.2027, found $=390.2113[\mathrm{M}+\mathrm{H}]^{+} .{ }^{1} \mathrm{H}$ NMR (500 MHz, D 6 -DMSO) $\delta: 9.00(\mathrm{~d}, J=1.5$ $\mathrm{Hz}, 1 \mathrm{H}), 8.49(\mathrm{~d}, J=8.1 \mathrm{~Hz}, 1 \mathrm{H}), 7.93\left(\mathrm{td}, J_{1}=8.8 \mathrm{~Hz}, J_{2}=5.9 \mathrm{~Hz}, 1 \mathrm{H}\right), 7.72\left(\mathrm{~m}, J_{1}=11.1\right.$ $\left.\mathrm{Hz}, J_{2}=8.9 \mathrm{~Hz}, J_{3}=2.7 \mathrm{~Hz}, 1 \mathrm{H}\right), 7.35-7.42(\mathrm{~m}, 1 \mathrm{H}), 3.71-3.82(\mathrm{~m}, 1 \mathrm{H}), 2.79-2.89(\mathrm{~m}, 2 \mathrm{H})$, 2.20-2.33 (m, 3 H), 1.69-1.81 (m, 6 H), 1.53-1.68 (m, 3 H), 1.14-1.28 (m, 4 H), 1.02-1.13 (m, $1 \mathrm{H})$.

\section{3-(2,4-Difluoro-phenyl)-isoxazole-5-carboxylic acid (1-cyclohexyl-piperidin-4-yl)-amide} (10c)

a) 2,4-Difluorobenzaldehyde oxime (4.25 g, $24.4 \mathrm{mmol})$ was dissolved in THF (50 mL). Then pyridine $(2.46 \mathrm{~mL}, 30.5 \mathrm{mmol})$ was added. The mixture was heated up to $60^{\circ} \mathrm{C}$ and $\mathrm{N}$ chlorosuccinimide $(3.58 \mathrm{~g}, 26.8 \mathrm{mmol})$ was added. The reaction mixture was stirred at $60^{\circ} \mathrm{C}$ for $45 \mathrm{~min}$ and then TEA $(4.11 \mathrm{~mL}, 29.2 \mathrm{mmol})$ and ethyl propiolate $(2.72 \mathrm{~mL}, 26.8 \mathrm{mmol})$ are added. The reaction mixture was stirred overnight at $60^{\circ} \mathrm{C}$ and then concentrated under $\mathrm{HV}$. The residue was taken up in DCM $(100 \mathrm{~mL})$ and diluted with aq. $1 \mathrm{M} \mathrm{HCl}(100 \mathrm{~mL})$. The separated organic phase was washed with water $(100 \mathrm{~mL})$. The organic phase was dried over $\mathrm{MgSO}_{4}$, filtered and the solvent was evaporated under $\mathrm{HV}$. The crude was purified by flash chromatography using n-heptane/EtOAc $9 / 1$ as eluent to yield ethyl 3-(2,4difluorophenyl)isoxazole-5-carboxylate $(3.8 \mathrm{~g}, 62 \%)$. LC-MS method A: $\mathrm{t}_{\mathrm{R}}=0.92 \mathrm{~min}$. 
b) 3-(2,4-Difluoro-phenyl)-isoxazole-5-carboxylic acid was obtained in quantitative yield by saponification of the above ester according to the procedure used for 10b. LC-MS method A: $\mathrm{t}_{\mathrm{R}}=0.68 \mathrm{~min} .{ }^{1} \mathrm{H}$ NMR (400 MHz, D6-DMSO) $\delta: 14.48(\mathrm{bs}, 1 \mathrm{H}), 7.99-8.05(\mathrm{~m}, 1 \mathrm{H}), 7.50-$ $7.56(\mathrm{~m}, 2 \mathrm{H}), 7.30(\mathrm{~m}, 1 \mathrm{H})$.

c) General procedure A from the above carboxylic acid and 1-cyclohexylpiperidin-4-ylamine to give 10c. QC LC-MS: $t_{R}=0.64 \mathrm{~min} ;[\mathrm{M}+\mathrm{H}]^{+}=390.3$. LC-HRMS: $\mathrm{t}_{\mathrm{R}}=0.57 \mathrm{~min} ; \mathrm{m} / \mathrm{z}=$ 389.1915, found $=390.1994[\mathrm{M}+\mathrm{H}]^{+} .{ }^{1} \mathrm{H}$ NMR $\left(500 \mathrm{MHz}, \mathrm{D}_{6}-\mathrm{DMSO}\right) \delta: 8.88(\mathrm{~d}, J=7.9$ $\mathrm{Hz}, 1 \mathrm{H}), 8.01\left(\mathrm{td}, J_{1}=6.6 \mathrm{~Hz}, J_{2}=8.7 \mathrm{~Hz}, 1 \mathrm{H}\right), 7.53\left(\mathrm{ddd}, J_{1}=2.4 \mathrm{~Hz}, J_{2}=9.0 \mathrm{~Hz}, J_{3}=11.4\right.$ $\mathrm{Hz}, 1 \mathrm{H}), 7.49(\mathrm{~d}, J=2.6 \mathrm{~Hz}, 1 \mathrm{H}), 7.30\left(\mathrm{td}, J_{1}=2.4 \mathrm{~Hz}, J_{2}=8.5 \mathrm{~Hz}, 1 \mathrm{H}\right), 3.66-3.77(\mathrm{~m}, 1 \mathrm{H})$, 2.80-2.88 (m, $2 \mathrm{H}), 2.20-2.32(\mathrm{~m}, 3 \mathrm{H}), 1.67-1.84(\mathrm{~m}, 6 \mathrm{H}), 1.48-1.62(\mathrm{~m}, 3 \mathrm{H}), 1.13-1.28$ (m, $4 \mathrm{H}), 0.98-1.13(\mathrm{~m}, 1 \mathrm{H})$.

\section{5-(2,4-Difluoro-phenyl)-[1,2,4]oxadiazole-3-carboxylic acid (1-cyclohexyl-piperidin-4-} yl)-amide (10d)

a) Ethyl 2-amino(hydroxyimino)acetate $(1.07 \mathrm{~g}, 8.1 \mathrm{mmol})$ dissolved in 2,6-dimethylpyridine $(2.93 \mathrm{~mL}, 24 \mathrm{mmol})$ was treated dropwise with a solution of 2,4-difluorobenzoyl chloride (0.66 $\mathrm{mL}, 5.38 \mathrm{mmol})$ in DCM $(15 \mathrm{~mL})$. The reaction mixture was stirred overnight. The beige suspension was dissolved with DCM $(150 \mathrm{~mL})$ and washed with water $(50 \mathrm{~mL})$, then $1 \mathrm{M} \mathrm{HCl}$ $(50 \mathrm{~mL})$ and brine $(50 \mathrm{~mL})$. The organic layer was dried over $\mathrm{MgSO}_{4}$, filtered and the solvent evaporated. The intermediate white powder ethyl 2-(2,4-difluorobenzamido)-2(hydroxyimino)acetate was then heated $1 \mathrm{~h}$ at $200^{\circ} \mathrm{C}$ in a DrySyn metal block (from Asynt Ltd.). After cooling down, the residue was purified by flash chromatography using a gradient of $2 \%$ to $20 \%$ EtOAc in n-heptane as eluent to yield ethyl 5-(2,4-difluorophenyl)-1,2,4oxadiazole-3-carboxylate $(1.20 \mathrm{~g}, 88 \%)$. LC-MS method A: $\mathrm{t}_{\mathrm{R}}=0.85 \mathrm{~min} ;[\mathrm{M}+\mathrm{H}]^{+}=255.02$. 
b) 5-(2,4-Difluoro-phenyl)-[1,2,4] oxadiazole-3-carboxylic acid was obtained by saponification of the above ester according to the procedure used for $10 \mathrm{~b}(0.83 \mathrm{~g}, 78 \%)$. LC-MS method A: $\mathrm{t}_{\mathrm{R}}=0.57 \mathrm{~min} .{ }^{1} \mathrm{H}$ NMR (400 MHz, D6-DMSO) $\delta: 8.27(\mathrm{~m}, 1 \mathrm{H}), 7.67(\mathrm{~m}, 1 \mathrm{H}), 7.41(\mathrm{~m}, 1 \mathrm{H})$.

c) General procedure A from the above carboxylic acid and 1-cyclohexylpiperidin-4-ylamine to give 10d. QC LC-MS: $t_{R}=0.58 \mathrm{~min} ;[\mathrm{M}+\mathrm{H}]^{+}=391.4$. LC-HRMS: $\mathrm{t}_{\mathrm{R}}=0.52 \mathrm{~min} ; \mathrm{m} / \mathrm{z}=$ 390.1867, found $=391.1943[\mathrm{M}+\mathrm{H}]^{+} .{ }^{1} \mathrm{H}$ NMR $\left(500 \mathrm{MHz}, \mathrm{D}_{6}\right.$-DMSO) $\delta: 8.95(\mathrm{~d}, J=8.1$ $\mathrm{Hz}, 1 \mathrm{H}), 8.28\left(\mathrm{td}, J_{1}=6.4 \mathrm{~Hz}, J_{2}=8.6 \mathrm{~Hz}, 1 \mathrm{H}\right), 7.67\left(\mathrm{ddd}, J_{1}=2.5 \mathrm{~Hz}, J_{2}=9.3 \mathrm{~Hz}, J_{3}=11.4\right.$ $\mathrm{Hz}, 1 \mathrm{H}), 7.39-7.44(\mathrm{~m}, 1 \mathrm{H}), 3.70-3.81(\mathrm{~m}, 1 \mathrm{H}), 2.83(\mathrm{~d}, J=11.4 \mathrm{~Hz}, 2 \mathrm{H}), 2.26(\mathrm{t}, J=10.5$ Hz, 3 H), 1.68-1.82 (m, 6 H), 1.54-1.65 (m, 3 H), 1.13-1.27 (m, 4 H), 0.99-1.12 (m, 1 H).

5-(2,4-Difluoro-phenyl)-[1,3,4]oxadiazole-2-carboxylic acid (1-cyclohexyl-piperidin-4yl)-amide (10e)

a) To a solution of 2,4-difluorobenzoic acid hydrazide $(2.65 \mathrm{~g}, 15.4 \mathrm{mmol})$ in $50 \mathrm{~mL}$ DCM was added TEA $(9.67 \mathrm{~mL}, 69.4 \mathrm{mmol})$. The mixture was cooled to $0^{\circ} \mathrm{C}$ and ethyl chlorooxoacetate (2.44 $\mathrm{mL}, 21.2 \mathrm{mmol})$ was added. The mixture was stirred $2 \mathrm{~h}$ at $0^{\circ} \mathrm{C}$. Then, toluene-4-sulfonyl chloride (4.40 g, $23.1 \mathrm{mmol})$ was added and stirring was continued overnight at rt. A sat. aq. $\mathrm{NaHCO}_{3}$ solution $(50 \mathrm{~mL})$ was added and the reaction mixture was extracted twice with DCM ( $2 \times 50 \mathrm{~mL})$. The combined organic layers were dried over $\mathrm{MgSO}_{4}$, filtered and evaporated. The residue was purified by flash chromatography using a gradient of eluent heptane/AcOEt (9:1 to 4:1) to give 5-(2,4-difluoro-phenyl)-[1,3,4]oxadiazole-2-carboxylic acid ethyl ester as a light yellow solid $(2.43 \mathrm{~g}, 60 \%)$. LC-MS method $\mathrm{A}: \mathrm{t}_{\mathrm{R}}=0.80 \mathrm{~min} ;[\mathrm{M}+\mathrm{H}]^{+}=255.13$, $[\mathrm{M}+\mathrm{H}+\mathrm{MeCN}]^{+}=296.10$.

b) To a solution of 1-cyclohexylpiperidin-4-amine ( $38 \mathrm{mg}, 0.2 \mathrm{mmol}$ ) in $0.4 \mathrm{~mL}$ toluene cooled to $0^{\circ} \mathrm{C}$ was added trimethylaluminium $2.0 \mathrm{M}$ in toluene $(0.1 \mathrm{~mL}, 0.2 \mathrm{mmol})$. After stirring for $30 \mathrm{~min}$. a solution of the above ester $(25.4 \mathrm{mg}, 0.1 \mathrm{mmol})$ in toluene $(0.4 \mathrm{~mL})$ was added. The reaction was stirred for $3 \mathrm{~h}$ and quenched with $1.25 \mathrm{M} \mathrm{HCl}$ in $\mathrm{MeOH}(0.240 \mathrm{~mL}, 0.3 \mathrm{mmol})$. 
The solvents were evaporated and the residue purified by prep. HPLC under basic conditions to yield 10e $(21 \mathrm{mg}, 54 \%)$. QC LC-MS: $\mathrm{t}_{\mathrm{R}}=0.57 \mathrm{~min} ;[\mathrm{M}+\mathrm{H}]+=391.3 . \mathrm{LC}-\mathrm{HRMS}: \mathrm{t}_{\mathrm{R}}=0.50$ $\min ; \mathrm{m} / \mathrm{z}=390.1867$, found $=391.1945[\mathrm{M}+\mathrm{H}]^{+} .{ }^{1} \mathrm{H}$ NMR $\left(500 \mathrm{MHz}, \mathrm{D}_{6}-\mathrm{DMSO}\right) \delta: 9.28$ $(\mathrm{d}, J=8.1 \mathrm{~Hz}, 1 \mathrm{H}), 8.18\left(\mathrm{td}, J_{1}=6.5 \mathrm{~Hz}, J_{2}=8.5 \mathrm{~Hz}, 1 \mathrm{H}\right), 7.63\left(\mathrm{ddd}, J_{1}=11.3 \mathrm{~Hz}, J_{2}=9.5\right.$ $\left.\mathrm{Hz}, J_{3}=2.4 \mathrm{~Hz}, 1 \mathrm{H}\right), 7.39\left(\mathrm{td}, J_{1}=2.1 \mathrm{~Hz}, J_{2}=8.3 \mathrm{~Hz}, 1 \mathrm{H}\right), 3.69-3.81(\mathrm{~m}, 1 \mathrm{H}), 2.84(\mathrm{~d}, J=$ 11.6 Hz, 2 H), 2.20-2.33 (m, 3 H), 1.67-1.83 (m, 6 H), 1.52-1.67 (m, 3 H), 1.13-1.29 (m, 4 H), 1.00-1.13 (m, $1 \mathrm{H})$.

6-(2,4-Difluoro-phenyl)-pyrimidine-4-carboxylic acid (1-cyclohexyl-piperidin-4-yl)amide (10f).

a) To a degassed solution of 2,4-difluorophenylboronic acid pinacol ester (1.47 g, $6.13 \mathrm{mmol})$ and methyl-6-chloropyrimidine-4-carboxylate $(0.96 \mathrm{~g}, 5.57 \mathrm{mmol})$ in DMF (15 mL) were added $\mathrm{K}_{3} \mathrm{PO}_{4}(1.67 \mathrm{~g}, 7.8 \mathrm{mmol})$ and $\mathrm{Pd}(\mathrm{dppf}) \mathrm{Cl}_{2} . \mathrm{DCM}(0.91 \mathrm{~g}, 1.11 \mathrm{mmol})$. The reaction mixture was stirred at $60^{\circ} \mathrm{C}$ for $18 \mathrm{~h}$. A sat. aq. $\mathrm{NaHCO}_{3}$ solution $(50 \mathrm{~mL})$ was added and the reaction mixture was extracted twice with DCM $(2 \times 50 \mathrm{~mL})$. The reaction mixture was diluted with $\mathrm{H}_{2} \mathrm{O}(100 \mathrm{~mL})$ and extracted with EtOAc $(2 \times 100 \mathrm{~mL})$. The combined organic layers were dried over $\mathrm{MgSO}_{4}$, filtered and evaporated. The residue was purified by flash chromatography using a gradient of heptane/AcOEt (7:3 to $1: 1)$ as eluent to give $6-(2,4-$ difluoro-phenyl)-pyrimidine-4-carboxylic acid methyl ester as a light yellow solid (0.96 g, $69 \%)$. LC-MS method A: $\mathrm{t}_{\mathrm{R}}=0.80 \mathrm{~min} ;[\mathrm{M}+\mathrm{H}]^{+}=251.08 ;{ }^{1} \mathrm{H} \mathrm{NMR}\left(400 \mathrm{MHz}, \mathrm{CDCl}_{3}\right) \delta$ : $9.46(\mathrm{~s}, 1 \mathrm{H}), 8.53(\mathrm{~s}, 1 \mathrm{H}), 8.31(\mathrm{q}, J=7.8 \mathrm{~Hz}, 1 \mathrm{H}), 7.11(\mathrm{t}, J=8.2 \mathrm{~Hz}, 1 \mathrm{H}), 7.02(\mathrm{~m}, 1 \mathrm{H})$, $4.10(\mathrm{~s}, 3 \mathrm{H})$.

b) 6-(2,4-difluoro-phenyl)-pyrimidine-4-carboxylic acid $(0.878 \mathrm{~g}, 97 \%)$ was obtained by saponification of the above ester according to the procedure used for 10b. LC-MS method A: $\mathrm{t}_{\mathrm{R}}=0.66 \mathrm{~min} ;[\mathrm{M}+\mathrm{H}]^{+}=237.07 ;{ }^{1} \mathrm{H} \mathrm{NMR}\left(400 \mathrm{MHz}, \mathrm{D}_{6}-\mathrm{DMSO}\right) \delta: 13.92-14.31($ broad s, 1 
H), $9.48(\mathrm{~s}, 1 \mathrm{H}), 8.34(\mathrm{~s}, 1 \mathrm{H}), 8.25(\mathrm{q}, J=8.0 \mathrm{~Hz}, 1 \mathrm{H}), 7.54(\mathrm{t}, J=10.8 \mathrm{~Hz}, 1 \mathrm{H}), 7.35(\mathrm{t}, J=$ $8.3 \mathrm{~Hz}, 1 \mathrm{H})$.

c) General procedure A from the above carboxylic acid and 1-cyclohexylpiperidin-4-ylamine to yield 10f. QC LC-MS: $\mathrm{t}_{\mathrm{R}}=0.64 \mathrm{~min} ;[\mathrm{M}+\mathrm{H}]^{+}=401.4$. LC-HRMS: $\mathrm{t}_{\mathrm{R}}=0.58 \mathrm{~min} ; \mathrm{m} / \mathrm{z}=$ 400.2075, found $=401.215[\mathrm{M}+\mathrm{H}]^{+} .{ }^{1} \mathrm{H}$ NMR $(400 \mathrm{MHz}, \mathrm{DMSO}) \delta$ : 9.38-9.43 $(\mathrm{m}, 1 \mathrm{H}), 8.83-$ 8.91 (m, $1 \mathrm{H}), 8.30-8.37$ (m, $1 \mathrm{H}), 8.18-8.29$ (m, $1 \mathrm{H})$, 7.45-7.56 (m, $1 \mathrm{H})$, 7.27-7.39 (m, $1 \mathrm{H})$, 3.71-3.85 (m, $1 \mathrm{H}), 2.77-2.89(\mathrm{~m}, 2 \mathrm{H}), 2.23-2.36(\mathrm{~m}, 3 \mathrm{H}), 1.52-1.81(\mathrm{~m}, 9 \mathrm{H}), 1.12-1.29(\mathrm{~m}$, $4 \mathrm{H}), 0.96-1.12(\mathrm{~m}, 1 \mathrm{H})$.

\section{Preparation of compounds 11a-11j}

\section{5-(2,4-Difluoro-phenyl)-isoxazole-3-carboxylic acid benzyl-(1-cyclohexyl-piperidin-4-yl)-}

amide (11a).1-Cyclohexylpiperidin-4-amine (18.2 mg, $0.1 \mathrm{mmol}, 1 \mathrm{eq})$, benzaldehyde (13 mg, $0.12 \mathrm{mmol})$ and $\mathrm{AcOH}(0.012 \mathrm{~mL}, 0.21 \mathrm{mmol})$ were dissolved in DCM (1 mL). Sodium triacetoxyborhydride (42.4 $\mathrm{mg}, 0.2 \mathrm{mmol}$ ) was added and the mixture was stirred overnight. Aq. $1 \mathrm{M} \mathrm{NaOH}(1 \mathrm{~mL})$ and DCM $(2 \mathrm{~mL})$ were added, the organic phase was separated, and the solvent evaporated under high vacuum. The obtained amine was then coupled according to general procedure A with 1-(2,4-difluorophenyl)-1H-pyrazole-3-carboxylic acid. LC-MS method B: $\mathrm{t}_{\mathrm{R}}=1.39 \mathrm{~min} ;[\mathrm{M}+\mathrm{H}]^{+}=480.1 . \mathrm{LC}-\mathrm{HRMS}: \mathrm{t}_{\mathrm{R}}=0.74 \mathrm{~min} ; \mathrm{m} / \mathrm{z}=479.2384$, found $=480.2471[\mathrm{M}+\mathrm{H}]^{+} .{ }^{1} \mathrm{H}$ NMR $\left(500 \mathrm{MHz}, \mathrm{D}_{6}\right.$-DMSO) $\delta: 7.93-8.14(\mathrm{~m}, 1 \mathrm{H}), 7.45-7.60(\mathrm{~m}, 1$ H), 6.99-7.37 (m, 7 H), 4.74 (s, 2 H), 3.78-4.25 (m, 1 H), 2.74-2.91 (m, 2 H), 2.13-2.28 (m, 2 H), $1.99-2.08(\mathrm{~m}, 1 \mathrm{H}), 1.60-1.76(\mathrm{~m}, 8 \mathrm{H}), 1.50-1.59(\mathrm{~m}, 1 \mathrm{H}), 1.07-1.23(\mathrm{~m}, 4 \mathrm{H}), 0.97-1.05$ (m, $1 \mathrm{H})$. 


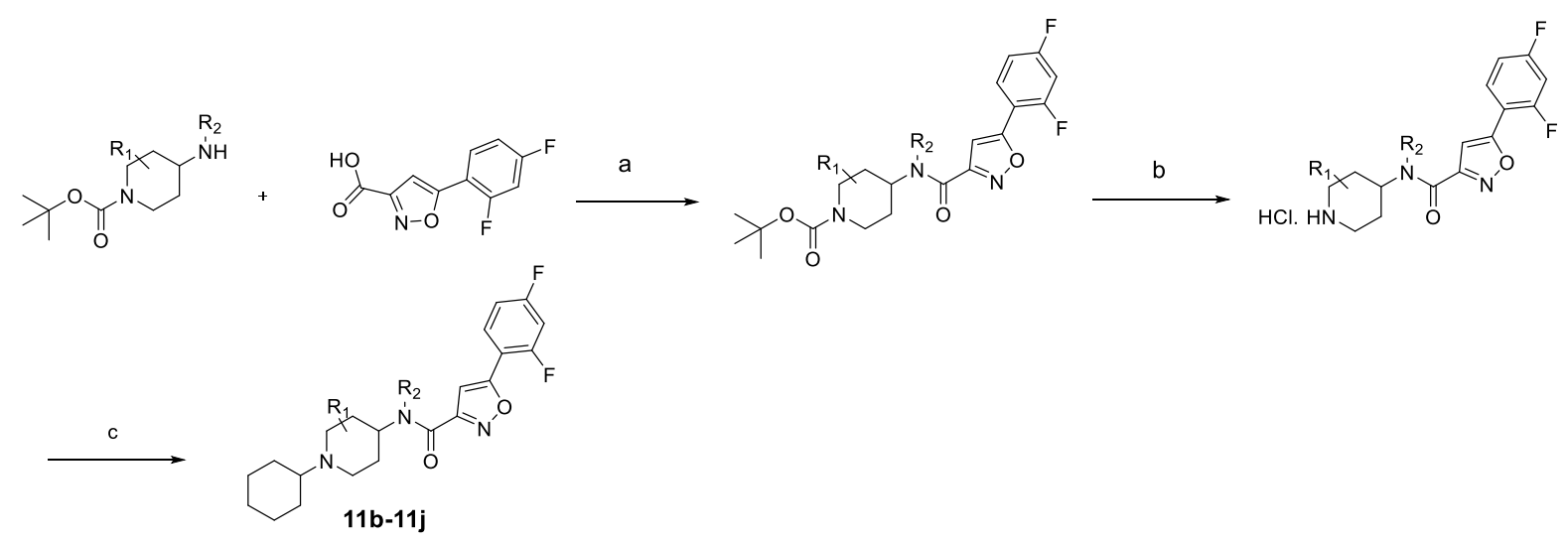

Reagents and conditions (a) HATU, DIPEA, DMF, rt, $18 \mathrm{~h}$; (b) $\mathrm{HCl} 4 \mathrm{~N}$ in dioxane, DCM, rt, 30 min; (c) cyclohexanone, $\mathrm{NaBH}(\mathrm{OAc})_{3}$, $\mathrm{AcOH}, \mathrm{DCM}, \mathrm{rt}, 24 \mathrm{~h}$.

\section{5-(2,4-Difluoro-phenyl)-isoxazole-3-carboxylic acid (1-cyclohexyl-3,3-difluoro-piperidin- 4-yl)-amide (11b)}

a) To a solution of 5-(2,4-difluorophenyl)isoxazole-3-carboxylic acid (67.5 $\mathrm{mg}, 0.3 \mathrm{mmol})$ in $2 \mathrm{~mL}$ DMF was added 4-amino-1-boc-3,3-difluoropiperidine (85 mg, $0.36 \mathrm{mmol}$ ). DIPEA (0.26 mL, $1.5 \mathrm{mmol})$ was then added followed by HATU (143 $\mathrm{mg}, 0.37 \mathrm{mmol})$. The reaction mixture was stirred overnight at rt. The crude mixture was directly purified by prep. LC-MS under basic conditions to yield tert-butyl 4-(5-(2,4-difluorophenyl)isoxazole-3-carboxamido)3,3-difluoropiperidine-1-carboxylate (89 mg, 67\%). LC-MS method $\mathrm{B}: \mathrm{t}_{\mathrm{R}}=1.64 \mathrm{~min}$; $\left[\mathrm{M}+\mathrm{NH}_{3}\right]^{+}=361.1$

b) The above-mentioned amide $(89 \mathrm{mg}, 0.2 \mathrm{mmol})$ was dissolved in DCM (1 mL). $\mathrm{HCl}$ in dioxane 4M (1 mL, $4 \mathrm{mmol})$ was added dropwise. The mixture was stirred at $\mathrm{rt}$ for 1 hour. The solvents were evaporated, and the residue was dried on high vacuum to deliver 5-(2,4difluorophenyl)-N-(3,3-difluoropiperidin-4-yl)isoxazole-3-carboxamide hydrochloride (77 $\mathrm{mg}, 100 \%$ ). LC-MS method B: $\mathrm{t}_{\mathrm{R}}=0.86 \mathrm{~min} ;[\mathrm{M}+\mathrm{H}]^{+}=344.0$.

c) To a suspension of the above-mentioned amine hydrochloride $(38 \mathrm{mg}, 0.1 \mathrm{mmol})$ in DCM $(0.5 \mathrm{~mL})$ at $\mathrm{rt}$ was added cyclohexanone $(15 \mathrm{mg}, 0.15 \mathrm{mmol})$ followed by acetic acid $(0.007$ $\mathrm{mL}, 0.125 \mathrm{mmol})$ and sodium triacetoxyborohydride $(32 \mathrm{~g}, 0.15 \mathrm{mmol})$. The reaction mixture 
was stirred overnight at $\mathrm{rt}$. The reaction mixture was evaporated. The crude compound was purified by prep. LC-MS under basic conditions to give 11b (14 mg, 33\%). LC-MS method B: $\mathrm{t}_{\mathrm{R}}=1.19 \mathrm{~min} ;[\mathrm{M}+\mathrm{H}]^{+}=426.1 . \mathrm{LC}-\mathrm{HRMS}: \mathrm{t}_{\mathrm{R}}=0.72 \mathrm{~min} ; \mathrm{m} / \mathrm{z}=425.1726$, found $=426.1807$ $[\mathrm{M}+\mathrm{H}]^{+} .{ }^{1} \mathrm{H}$ NMR $(400 \mathrm{MHz}, \mathrm{DMSO}$, solvent suppression) $\delta: 8.97(\mathrm{~d}, J=8.8 \mathrm{~Hz}, 1 \mathrm{H}), 8.06$ $\left(\mathrm{td}, J_{1}=8.6 \mathrm{~Hz}, J_{2}=6.4 \mathrm{~Hz}, 1 \mathrm{H}\right), 7.57\left(\mathrm{ddd}, J_{1}=11.5 \mathrm{~Hz}, J_{2}=9.3 \mathrm{~Hz}, J_{3}=2.4 \mathrm{~Hz}, 1 \mathrm{H}\right), 7.30-$ $7.36(\mathrm{~m}, 1 \mathrm{H}), 7.23(\mathrm{~d}, J=2.8 \mathrm{~Hz}, 1 \mathrm{H}), 4.25-4.44(\mathrm{~m}, 1 \mathrm{H}), 3.05-3.13(\mathrm{~m}, 1 \mathrm{H}), 2.78-2.91(\mathrm{~m}$, $1 \mathrm{H}), 2.55-2.76(\mathrm{~m}, 1 \mathrm{H}), 2.34-2.45(\mathrm{~m}, 2 \mathrm{H}), 1.66-1.90(\mathrm{~m}, 6 \mathrm{H}), 1.52-1.63(\mathrm{~m}, 1 \mathrm{H}), 1.12-1.29$ (m, $4 \mathrm{H}), 1.01-1.12(\mathrm{~m}, 1 \mathrm{H})$.

Rac-5-(2,4-Difluoro-phenyl)-isoxazole-3-carboxylic acid $\quad\left(\left(3 S^{*}, 4 S^{*}\right)-1-c y c l o h e x y l-3-\right.$ methoxy-piperidin-4-yl)-amide (11c). Prepared in analogy to compound 11b from tert-butyl trans-4-amino-3-methoxy-piperidine-1-carboxylate. LC-MS method $\mathrm{B}: \mathrm{t}_{\mathrm{R}}=1.09 \mathrm{~min} ;[\mathrm{M}+\mathrm{H}]^{+}$ $=420.2$. LC-HRMS: $\mathrm{t}_{\mathrm{R}}=0.60 \mathrm{~min} ; \mathrm{m} / \mathrm{z}=419.2020$, found $=420.21[\mathrm{M}+\mathrm{H}]^{+} .{ }^{1} \mathrm{H}$ NMR $(500$ MHz, D6-DMSO) $\delta: 8.81(\mathrm{~d}, J=8.6 \mathrm{~Hz}, 1 \mathrm{H}), 8.06\left(\mathrm{td}, J_{1}=6.4 \mathrm{~Hz}, J_{2}=8.7 \mathrm{~Hz}, 1 \mathrm{H}\right), 7.59$ $\left(\mathrm{ddd}, J_{1}=2.5 \mathrm{~Hz}, J_{2}=9.3 \mathrm{~Hz}, J_{3}=11.5 \mathrm{~Hz}, 1 \mathrm{H}\right), 7.34\left(\mathrm{td}, J_{1}=2.2 \mathrm{~Hz}, J_{2}=8.3 \mathrm{~Hz}, 1 \mathrm{H}\right), 7.15$ $(\mathrm{d}, J=2.9 \mathrm{~Hz}, 1 \mathrm{H}), 3.63-3.74(\mathrm{~m}, 1 \mathrm{H}), 3.30(\mathrm{~s}, 3 \mathrm{H}), 3.14-3.23(\mathrm{~m}, 1 \mathrm{H}), 2.71-2.79(\mathrm{~m}, 1 \mathrm{H})$, 2.50-2.55 (m, 2 H), 2.30-2.36 (m, $1 \mathrm{H}), 2.21(\mathrm{~m}, 1 \mathrm{H}), 1.96(\mathrm{t}, J=10.4 \mathrm{~Hz}, 1 \mathrm{H}), 1.69-1.81$ (m, 4 H), 1.46-1.62 (m, 2 H), 1.14-1.27 (m, 4 H), 1.02-1.12 (m, 1 H).

Rac-5-(2,4-Difluoro-phenyl)-isoxazole-3-carboxylic acid $\quad\left(\left(3 S^{*}, 4 S^{*}\right)-1-c y c l o h e x y l-3-\right.$ ethoxy-piperidin-4-yl)-amide (11d). Prepared in analogy to compound $\mathbf{1 1 b}$ from tert-butyl trans-4-amino-3-ethoxy-piperidine-1-carboxylate. LC-MS method B: $\mathrm{t}_{\mathrm{R}}=1.16 \mathrm{~min} ;[\mathrm{M}+\mathrm{H}]^{+}$ $=434.1 . \mathrm{LC}-\mathrm{HRMS}: \mathrm{t}_{\mathrm{R}}=0.64 \mathrm{~min} ; \mathrm{m} / \mathrm{z}=433.2177$, found $=434.2258[\mathrm{M}+\mathrm{H}]^{+} .{ }^{1} \mathrm{H} \mathrm{NMR}(400$ MHz, DMSO, solvent suppression) $\delta: 8.77(\mathrm{~d}, J=8.6 \mathrm{~Hz}, 1 \mathrm{H}), 8.01-8.12(\mathrm{~m}, 1 \mathrm{H}), 7.53-7.62$ (m, $1 \mathrm{H}), 7.28-7.39(\mathrm{~m}, 1 \mathrm{H}), 7.13(\mathrm{~d}, J=2.7 \mathrm{~Hz}, 1 \mathrm{H}), 3.52-3.73(\mathrm{~m}, 1 \mathrm{H}), 3.05-3.14(\mathrm{~m}, 1 \mathrm{H})$, 2.66-2.80 (m, $1 \mathrm{H}), 2.26-2.39(\mathrm{~m}, 1 \mathrm{H}), 2.14-2.26(\mathrm{~m}, 1 \mathrm{H}), 1.94-2.05(\mathrm{~m}, 1 \mathrm{H}), 1.62-1.82(\mathrm{~m}$, $5 \mathrm{H}), 1.46-1.62$ (m, $2 \mathrm{H}), 1.12-1.25(\mathrm{~m}, 4 \mathrm{H}), 0.97-1.12(\mathrm{~m}, 4 \mathrm{H})$. 
Rac-5-(2,4-Difluoro-phenyl)-isoxazole-3-carboxylic acid $\quad\left(\left(3 S^{*}, 4 S^{*}\right)-1-c y c l o h e x y l-3-\right.$ hydroxy-piperidin-4-yl)-amide (11e). Prepared in analogy to compound 11b from tert-butyl trans-4-amino-3-hydroxy-piperidine-1-carboxylate. QC LC-MS: $\mathrm{t}_{\mathrm{R}}=0.55 \mathrm{~min} ;[\mathrm{M}+\mathrm{H}]^{+}=$ 406.2. LC-HRMS: $\mathrm{t}_{\mathrm{R}}=0.55 \mathrm{~min} ; \mathrm{m} / \mathrm{z}=405.1864$, found $=406.1939[\mathrm{M}+\mathrm{H}]^{+} .{ }^{1} \mathrm{H} \mathrm{NMR}(400$ $\left.\mathrm{MHz}, \mathrm{CDCl}_{3}\right) \delta: 7.96\left(\mathrm{td}, J_{1}=6.3 \mathrm{~Hz}, J_{2}=8.5 \mathrm{~Hz}, 1 \mathrm{H}\right), 7.12(\mathrm{~d}, J=3.7 \mathrm{~Hz}, 1 \mathrm{H}), 6.97-7.10$ $(\mathrm{m}, 3 \mathrm{H}), 6.90(\mathrm{~d}, J=7.3 \mathrm{~Hz}, 1 \mathrm{H}), 3.85-3.95(\mathrm{~m}, 1 \mathrm{H}), 3.68\left(\mathrm{td}, J_{1}=4.2 \mathrm{~Hz}, J_{2}=8.7 \mathrm{~Hz}, 1 \mathrm{H}\right)$, 3.08-3.16 (m, $1 \mathrm{H}), 2.81-2.90(\mathrm{~m}, 1 \mathrm{H}), 2.27-2.48(\mathrm{~m}, 3 \mathrm{H}), 2.07-2.16(\mathrm{~m}, 1 \mathrm{H}), 1.76-1.91(\mathrm{~m}$, 4 H), 1.60-1.72 (m, 2 H), 1.19-1.34 (m, 4 H), 1.05-1.19 (m, 1 H).

Rac-5-(2,4-Difluoro-phenyl)-isoxazole-3-carboxylic acid $\quad\left(\left(3 S^{*}, 4 S^{*}\right)-1-c y c l o h e x y l-3-\right.$ hydroxymethyl-piperidin-4-yl)-amide (11f). To a solution of ester $11 \mathrm{~g}(0.67 \mathrm{~g}, 1.5 \mathrm{mmol})$ in $17.1 \mathrm{~mL}$ dry THF was added $\mathrm{LiCl}(0.51 \mathrm{~g}, 12 \mathrm{mmol})$, followed by $\mathrm{NaBH}_{4}(0.23 \mathrm{~g}, 6 \mathrm{mmol})$ and $8 \mathrm{~mL}$ dry EtOH. After stirring for $44 \mathrm{~h}, 20 \mathrm{~mL}$ water was added, THF and EtOH were evaporated under high vacuum and the mixture was diluted with $50 \mathrm{~mL}$ DCM. The organic phase was separated dried over $\mathrm{Na}_{2} \mathrm{SO}_{4}$ and the solvent evaporated under high vacuum to yield $11 f(0.50 \mathrm{~g}, 79 \%)$. LC-MS method $B t_{R}=1.03 \mathrm{~min} ;[\mathrm{M}+\mathrm{H}]^{+}=420.23$. LC-HRMS: $\mathrm{t}_{\mathrm{R}}=0.56$ $\min ; \mathrm{m} / \mathrm{z}=419.2020$, found $=420.2098[\mathrm{M}+\mathrm{H}]^{+} .{ }^{1} \mathrm{H}$ NMR $\left(500 \mathrm{MHz}, \mathrm{D}_{6}-\mathrm{DMSO}\right) \delta: 8.73$ $(\mathrm{d}, J=8.7 \mathrm{~Hz}, 1 \mathrm{H}), 8.06\left(\mathrm{td}, J_{1}=6.5 \mathrm{~Hz}, J_{2}=8.7 \mathrm{~Hz}, 1 \mathrm{H}\right), 7.59\left(\mathrm{ddd}, J_{1}=2.5 \mathrm{~Hz}, J_{2}=9.3\right.$ $\left.\mathrm{Hz}, J_{3}=11.5 \mathrm{~Hz}, 1 \mathrm{H}\right), 7.34\left(\mathrm{td}, J_{1}=2.1 \mathrm{~Hz}, J_{2}=8.3 \mathrm{~Hz}, 1 \mathrm{H}\right), 7.16(\mathrm{~d}, J=2.9 \mathrm{~Hz}, 1 \mathrm{H}), 4.40$ $(\mathrm{t}, J=5.0 \mathrm{~Hz}, 1 \mathrm{H}), 3.52-3.62(\mathrm{~m}, 1 \mathrm{H}), 3.43-3.50(\mathrm{~m}, 1 \mathrm{H}), 3.16-3.24(\mathrm{~m}, 1 \mathrm{H}), 3.03-3.09(\mathrm{~m}$, $1 \mathrm{H}), 2.78-2.85(\mathrm{~m}, 1 \mathrm{H}), 2.17-2.32(\mathrm{~m}, 2 \mathrm{H}), 1.99(\mathrm{t}, J=11.1 \mathrm{~Hz}, 1 \mathrm{H}), 1.69-1.82(\mathrm{~m}, 6 \mathrm{H})$, 1.54-1.66 (m, 2 H), 1.14-1.27 (m, $4 \mathrm{H}), 1.02-1.13$ (m, $1 \mathrm{H})$.

\section{1-Cyclohexyl-4-\{[5-(2,4-difluoro-phenyl)-isoxazole-3-carbonyl]-amino\}-piperidine-4-}

carboxylic acid amide (11j). Prepared in analogy to compound $\mathbf{1 1 b}$ from tert-butyl 4-amino4-carbamoylpiperidine-1-carboxylate. QC LC-MS: $\mathrm{t}_{\mathrm{R}}=0.64 \mathrm{~min} ;[\mathrm{M}+\mathrm{H}]^{+}=433.4$. LCHRMS: $\mathrm{t}_{\mathrm{R}}=0.57 \mathrm{~min} ; \mathrm{m} / \mathrm{z}=432.1973$, found $=433.2047[\mathrm{M}+\mathrm{H}]^{+} .{ }^{1} \mathrm{H}$ NMR $\left(400 \mathrm{MHz}, \mathrm{D}_{6^{-}}\right.$ 
DMSO) $\delta: 8.14(\mathrm{~s}, 1 \mathrm{H}), 8.06\left(\mathrm{td}, J_{1}=6.6 \mathrm{~Hz}, J_{2}=8.6 \mathrm{~Hz}, 1 \mathrm{H}\right), 7.58\left(\mathrm{ddd}, J_{1}=2.4 \mathrm{~Hz}, J_{2}=\right.$ $\left.9.5 \mathrm{~Hz}, J_{3}=11.5 \mathrm{~Hz}, 1 \mathrm{H}\right), 7.31-7.37(\mathrm{~m}, 1 \mathrm{H}), 7.19(\mathrm{~d}, J=2.8 \mathrm{~Hz}, 2 \mathrm{H}), 6.93(\mathrm{~s}, 1 \mathrm{H}), 2.66(\mathrm{~d}$, $J=11.7 \mathrm{~Hz}, 2 \mathrm{H}), 2.37$ (t, $J=11.2 \mathrm{~Hz}, 2 \mathrm{H}), 2.10-2.25$ (m, $3 \mathrm{H}), 1.86-1.98$ (m, $2 \mathrm{H}), 1.65-1.77$ (m, $4 \mathrm{H}), 1.51-1.59(\mathrm{~m}, 1 \mathrm{H}), 1.10-1.25(\mathrm{~m}, 4 \mathrm{H}), 1.00-1.10(\mathrm{~m}, 1 \mathrm{H})$.

\section{$\underline{\text { Preparation of racemic ester } \mathbf{1 1 g} \text {, and carboxylic acid } \mathbf{1 1 h}}$}

\section{$r a c-\left(3 R^{*}, 4 R^{*}\right)-1-C y c l o h e x y l-4-\{[5-(2,4-d i f l u o r o-p h e n y l)-i s o x a z o l e-3-c a r b o n y l]-a m i n o\}-$ piperidine-3-carboxylic acid methyl ester (11g)}

a) To a solution of $r a c-\left(3 R^{*}, 4 R^{*}\right)-4$-tert-butoxycarbonylamino-piperidine-3-carboxylic acid methyl ester $(3.0 \mathrm{~g}, 11.3 \mathrm{mmol})$ in DCM $(56 \mathrm{~mL})$ was added cyclohexanone $(1.42 \mathrm{~mL}, 13.5$ mmol) followed by acetic acid $(0.966 \mathrm{~mL}, 16.9 \mathrm{mmol})$ and sodium triacetoxyborohydride $(3.39$ g, $15.2 \mathrm{mmol})$. After stirring for $5 \mathrm{~h}$, additional cyclohexanone $(0.23 \mathrm{~mL}, 2.3 \mathrm{mmol})$, acetic acid $(0.17 \mathrm{~mL}, 2.8 \mathrm{mmol})$ and sodium triacetoxyborohydride $(0.59 \mathrm{~g}, 2.8 \mathrm{mmol})$ were added. The reaction mixture was stirred overnight. The reaction mixture was diluted with DCM $(200 \mathrm{~mL})$ and treated with aq. sat. $\mathrm{NaHCO}_{3}(250 \mathrm{~mL})$. The organic phase was dried over $\mathrm{MgSO}_{4}$ and evaporated. Compound rac-methyl $\quad\left(3 R^{*}, 4 R^{*}\right)-4-(($ tert-butoxycarbonyl)amino)-1cyclohexylpiperidine-3-carboxylate was used in the next step without further purification (3.85 g, 100\%); LC-MS method B $t_{R}=1.09$ min; $[\mathrm{M}+\mathrm{H}]^{+}=341.19$.

b) The above-mentioned compound (3.85 g, $11.3 \mathrm{mmol})$ was dissolved in $\mathrm{MeOH}(56.5 \mathrm{~mL})$. A $4 \mathrm{M}$ solution of $\mathrm{HCl}$ in dioxane $(56.5 \mathrm{~mL}, 226 \mathrm{mmol})$ was added and the reaction was stirred for $1 \mathrm{~h}$. The reaction mixture was concentrated, dissolved in DCM $(250 \mathrm{~mL})$ and treated with aq. sat. $\mathrm{NaHCO}_{3}(200 \mathrm{~mL})$. The organic layer was separated, and the aqueous phase was extracted with DCM $(150 \mathrm{~mL})$. The combined organic layers were dried over $\mathrm{MgSO}_{4}$ and evaporated. The crude rac-methyl $\left(3 R^{*}, 4 R^{*}\right)$-4-amino-1-cyclohexylpiperidine-3-carboxylate hydrochloride was obtained as a yellow oil $(2.64 \mathrm{~g}, 92 \%)$; LC-MS method $\mathrm{B} \mathrm{t}_{\mathrm{R}}=0.79 \mathrm{~min}$; 
$[\mathrm{M}+\mathrm{H}]^{+}=241.20 .{ }^{1} \mathrm{H}$ NMR $(400 \mathrm{MHz}, \mathrm{CDCl} 3) \delta: 7.28$ (s, $\left.1 \mathrm{H}\right), 3.61-3.79(\mathrm{~m}, 4 \mathrm{H}), 3.06-3.13$ (m, $1 \mathrm{H}), 2.83-2.92(\mathrm{~m}, 2 \mathrm{H}), 2.23-2.44(\mathrm{~m}, 4 \mathrm{H}), 1.04-1.93(\mathrm{~m}, 18 \mathrm{H})$.

c) General procedure A from the above amine hydrochloride and 5-(2,4difluorophenyl)isoxazole-3-carboxylic acid to yield 11g. LC-MS method B: $t_{R}=1.15$ min; $[\mathrm{M}+\mathrm{H}]^{+}=447.92$. LC-HRMS: $\mathrm{t}_{\mathrm{R}}=0.60 \mathrm{~min} ; \mathrm{m} / \mathrm{z}=447.1970$, found $=448.2046[\mathrm{M}+\mathrm{H}]^{+} .{ }^{1} \mathrm{H}$ NMR (400 MHz, $\left.\mathrm{CDCl}_{3}\right) \delta: 7.97\left(\mathrm{td}, J_{1}=6.3 \mathrm{~Hz}, J_{2}=8.5 \mathrm{~Hz}, 1 \mathrm{H}\right), 7.11(\mathrm{~d}, J=3.8 \mathrm{~Hz}, 1 \mathrm{H})$, 7.04-7.10 (m, $1 \mathrm{H}), 7.01\left(\mathrm{ddd}, J_{1}=2.4 \mathrm{~Hz}, J_{2}=8.6 \mathrm{~Hz}, J_{3}=10.8 \mathrm{~Hz}, 1 \mathrm{H}\right), 6.82(\mathrm{~d}, J=8.5 \mathrm{~Hz}$, $1 \mathrm{H}), 4.20-4.31(\mathrm{~m}, 1 \mathrm{H}), 3.69(\mathrm{~m}, 3 \mathrm{H}), 3.05-3.15(\mathrm{~m}, 1 \mathrm{H}), 2.90-2.98(\mathrm{~m}, 1 \mathrm{H}), 2.65-2.74(\mathrm{~m}$, $1 \mathrm{H}), 2.56-2.65(\mathrm{~m}, 1 \mathrm{H}), 2.44-2.53(\mathrm{~m}, 1 \mathrm{H}), 2.33-2.43(\mathrm{~m}, 1 \mathrm{H}), 2.15-2.23(\mathrm{~m}, 1 \mathrm{H}), 1.73-1.91$ (m, 4 H), 1.57-1.72 (m, 2 H), 1.18-1.37 (m, 4 H), 1.06-1.19 (m, 1 H).

\section{$R a c-\left(3 R^{*}, 4 R^{*}\right)-1-C y c l o h e x y l-4-\{[5-(2,4-d i f l u o r o-p h e n y l)-i s o x a z o l e-3-c a r b o n y l]-a m i n o\}-$}

piperidine-3-carboxylic acid (11h). Prepared in analogy to compound 19 from ester 11g. LCMS method B: $t_{R}=0.59 \min ;[M+H]^{+}=433.82$. LC-HRMS: $t_{R}=0.62 \mathrm{~min} ; \mathrm{m} / \mathrm{z}=433.1813$, found $=434.1896[\mathrm{M}+\mathrm{H}]^{+} .{ }^{1} \mathrm{H}$ NMR $\left(400 \mathrm{MHz}, \mathrm{D}_{6}-\mathrm{DMSO}\right) \delta: 11.76-12.73(\mathrm{~m}, 1 \mathrm{H}), 8.92(\mathrm{~d}$, $J=8.1 \mathrm{~Hz}, 1 \mathrm{H}), 8.08\left(\mathrm{td}, J_{1}=8.8 \mathrm{~Hz}, J_{2}=6.5 \mathrm{~Hz}, 1 \mathrm{H}\right), 7.61\left(\mathrm{ddd}, J_{1}=2.5 \mathrm{~Hz}, J_{2}=9.3 \mathrm{~Hz}, J_{3}\right.$ $=11.5 \mathrm{~Hz}, 1 \mathrm{H}), 7.32-7.38(\mathrm{~m}, 1 \mathrm{H}), 7.15(\mathrm{~d}, J=3.0 \mathrm{~Hz}, 1 \mathrm{H}), 4.00-4.14(\mathrm{~m}, 1 \mathrm{H}), 3.03-3.16$ (m, $1 \mathrm{H}), 2.84-2.98(\mathrm{~m}, 1 \mathrm{H}), 2.68-2.80(\mathrm{~m}, 1 \mathrm{H}), 2.32-2.54(\mathrm{~m}, 3 \mathrm{H}), 1.70-1.90(\mathrm{~m}, 5 \mathrm{H}), 1.52-$ 1.69 (m, 2 H), 1.18-1.24 (m, 4 H), 1.07-1.10 (m, $1 \mathrm{H})$.

\section{$R a c-\left(3 R^{*}, 4 R^{*}\right)-1-C y c l o h e x y l-4-\{[5-(2,4-d i f l u o r o-p h e n y l)-i s o x a z o l e-3-c a r b o n y l]-a m i n o\}-$}

piperidine-3-carboxylic acid dimethylamide (11i). Prepared in analogy to compound $(3 S, 4 S)$-11i from carboxylic acid 11h. $\mathrm{LC}-\mathrm{MS}$ method B: $\mathrm{t}_{\mathrm{R}}=1.0 \mathrm{~min} ;[\mathrm{M}+\mathrm{H}]^{+}=460.95$. LCHRMS: $\mathrm{t}_{\mathrm{R}}=0.59 \mathrm{~min} ; \mathrm{m} / \mathrm{z}=460.2286$, found $=461.2372[\mathrm{M}+\mathrm{H}]^{+} .{ }^{1} \mathrm{H}$ NMR $\left(500 \mathrm{MHz}, \mathrm{D}_{6-}\right.$ DMSO) $\delta: 8.68(\mathrm{~d}, J=8.5 \mathrm{~Hz}, 1 \mathrm{H}), 8.05\left(\mathrm{td}, J_{1}=8.7 \mathrm{~Hz}, J_{2}=6.5 \mathrm{~Hz}, 1 \mathrm{H}\right), 7.58\left(\mathrm{ddd}, J_{1}=\right.$ $\left.11.5 \mathrm{~Hz}, J_{2}=9.3 \mathrm{~Hz}, J_{3}=2.4 \mathrm{~Hz}, 1 \mathrm{H}\right), 7.33\left(\mathrm{td}, J_{1}=8.3 \mathrm{~Hz}, J_{2}=2.1 \mathrm{~Hz}, 1 \mathrm{H}\right), 7.09(\mathrm{~d}, J=2.9$ $\mathrm{Hz}, 1 \mathrm{H}), 4.03-4.13(\mathrm{~m}, 1 \mathrm{H}), 3.13\left(\mathrm{td}, J_{1}=10.7 \mathrm{~Hz}, J_{2}=3.5 \mathrm{~Hz}, 1 \mathrm{H}\right), 3.06(\mathrm{~s}, 3 \mathrm{H}), 2.80-2.91$ 
(m, 2 H), 2.77 (s, $3 \mathrm{H}), 2.23-2.36(\mathrm{~m}, 2 \mathrm{H}), 2.19(\mathrm{t}, J=11.2 \mathrm{~Hz}, 1 \mathrm{H}), 1.79-1.87(\mathrm{~m}, 1 \mathrm{H}), 1.67-$ 1.79 (m, $4 \mathrm{H}), 1.53-1.64$ (m, $2 \mathrm{H}), 1.13-1.28$ (m, $4 \mathrm{H}), 0.99-1.13(\mathrm{~m}, 1 \mathrm{H})$.

\section{(3R,4R)-1-Cyclohexyl-4-\{[5-(2,4-difluoro-phenyl)-isoxazole-3-carbonyl]-amino\}-} piperidine-3-carboxylic acid dimethylamide $(3 R, 4 R)-11$. Prepared in analogy to compound $(3 S, 4 S)-11 \mathbf{i}$, starting from $(3 R, 4 R)-4-((R)-1$-phenyl-ethylamino)-piperidine-1,3-dicarboxylic acid 1-tert-butyl ester 3-ethyl ester $\mathbf{1 9}^{39}$. LC-MS method A: $\mathrm{t}_{\mathrm{R}}=0.71 \mathrm{~min} ;[\mathrm{M}+\mathrm{H}]^{+}=461.23$. Chiral HPLC: $t_{\mathrm{R}}=9.1 \mathrm{~min} ; 95.6 \%$ ee; Column: Regis $(\mathrm{R}, \mathrm{R})$ Whelk-O1, 4.6x250 mm, $5 \mu \mathrm{m}$; Detector wavelength: 254 nm; Eluent: 30\% Heptane 0.05\% DEA; 70\% Ethanol 0.05\% DEA; Flow: $0.8 \mathrm{~mL} / \mathrm{min}$; BPR: 150 bar; Temperature: $25^{\circ} \mathrm{C}$. Injection volume: $2.5 \mu 1$. LC-HRMS: $\mathrm{t}_{\mathrm{R}}$ $=0.60 \mathrm{~min} ; \mathrm{m} / \mathrm{z}=460.2285$, found $=461.2362[\mathrm{M}+\mathrm{H}]^{+} .{ }^{1} \mathrm{H}$ NMR $\left(400 \mathrm{MHz}, \mathrm{D}_{6}-\mathrm{DMSO}\right) \delta$ : $8.69(\mathrm{~d}, J=8.3 \mathrm{~Hz}, 1 \mathrm{H}), 8.05(\mathrm{q}, J=7.9 \mathrm{~Hz}, 1 \mathrm{H}), 7.58(\mathrm{~m}, 1 \mathrm{H}), 7.33(\mathrm{t}, J=8.4 \mathrm{~Hz}, 1 \mathrm{H})$, 7.09-7.11 (m, $1 \mathrm{H}), 4.03-4.12(\mathrm{~m}, 1 \mathrm{H}), 3.13(\mathrm{~m}, 2 \mathrm{H}), 3.06(\mathrm{~s}, 3 \mathrm{H}), 2.85(\mathrm{t}, J=13.5 \mathrm{~Hz}, 1 \mathrm{H})$, 2.77 (s, $3 \mathrm{H}), 2.16-2.33(\mathrm{~m}, 3 \mathrm{H}), 1.83$ (d, $J=11.0 \mathrm{~Hz}, 1 \mathrm{H}), 1.73(\mathrm{~d}, J=7.2 \mathrm{~Hz}, 4 \mathrm{H}), 1.57$ (d, $J=12.0 \mathrm{~Hz}, 2 \mathrm{H}), 1.12-1.26(\mathrm{~m}, 4 \mathrm{H}), 1.04-1.11(\mathrm{~m}, 1 \mathrm{H})$.

\section{Preparation of compounds 20a-20q and 21a-21g}

The compounds were prepared in analogy to compound $(3 S, 4 S)-11$ from carboxylic acid $11 \mathbf{h}$ and the corresponding amine.

\section{$R a c-\left(3 R^{*}, 4 R^{*}\right)-1-C y c l o h e x y l-4-\{[5-(2,4-d i f l u o r o-p h e n y l)-i s o x a z o l e-3-c a r b o n y l]-a m i n o\}-$} piperidine-3-carboxylic acid amide (20a). Prepared from ammonium chloride. LC-MS method $\mathrm{B}: \mathrm{t}_{\mathrm{R}}=0.88 \mathrm{~min} ;[\mathrm{M}+\mathrm{H}]^{+}=433.18 . \mathrm{LC}-\mathrm{HRMS}: \mathrm{t}_{\mathrm{R}}=0.53 \mathrm{~min} ; \mathrm{m} / \mathrm{z}=432.1973$, found $=433.205[\mathrm{M}+\mathrm{H}]^{+} .{ }^{1} \mathrm{H}$ NMR $\left(500 \mathrm{MHz}, \mathrm{D}_{6}-\mathrm{DMSO}\right) \delta: 8.60(\mathrm{~d}, J=8.2 \mathrm{~Hz}, 1 \mathrm{H}), 8.05\left(\mathrm{td}, J_{1}\right.$ $\left.=6.5 \mathrm{~Hz}, J_{2}=8.7 \mathrm{~Hz}, 1 \mathrm{H}\right), 7.58\left(\mathrm{ddd}, J_{1}=2.5 \mathrm{~Hz}, J_{2}=9.3 \mathrm{~Hz}, J_{3}=11.5 \mathrm{~Hz}, 1 \mathrm{H}\right), 7.33\left(\mathrm{td}, J_{1}\right.$ $\left.=2.1 \mathrm{~Hz}, J_{2}=8.3 \mathrm{~Hz}, 1 \mathrm{H}\right), 7.16(\mathrm{~s}, 1 \mathrm{H}), 7.12(\mathrm{~d}, J=2.8 \mathrm{~Hz}, 1 \mathrm{H}), 6.83(\mathrm{~s}, 1 \mathrm{H}), 3.90-4.03(\mathrm{~m}$, 
$1 \mathrm{H}), 2.87-2.95(\mathrm{~m}, 1 \mathrm{H}), 2.77-2.86(\mathrm{~m}, 1 \mathrm{H}), 2.55-2.63(\mathrm{~m}, 1 \mathrm{H}), 2.21-2.35(\mathrm{~m}, 3 \mathrm{H}), 1.64-1.89$ (m, 5 H), 1.42-1.63 (m, 2 H), 1.14-1.30 (m, 5 H), 0.98-1.13 (m, 1 H).

\section{Rac-(3R*,4R*)-1-Cyclohexyl-4-\{[5-(2,4-difluoro-phenyl)-isoxazole-3-carbonyl]-amino $\}$ -} piperidine-3-carboxylic acid methylamide (20b). Prepared from methylamine hydrochloride. LC-MS method $\mathrm{B}: \mathrm{t}_{\mathrm{R}}=0.95 \mathrm{~min} ;[\mathrm{M}+\mathrm{H}]^{+}=446.95 . \mathrm{LC}-\mathrm{HRMS}: \mathrm{t}_{\mathrm{R}}=0.55 \mathrm{~min}$; $\mathrm{m} / \mathrm{z}=446.2129$, found $=447.2209[\mathrm{M}+\mathrm{H}]^{+} .{ }^{1} \mathrm{H}$ NMR $\left(500 \mathrm{MHz}, \mathrm{D}_{6}-\mathrm{DMSO}\right) \delta: 8.57(\mathrm{~d}, J=$ $8.4 \mathrm{~Hz}, 1 \mathrm{H}), 8.05\left(\mathrm{td}, J_{1}=6.4 \mathrm{~Hz}, J_{2}=8.7 \mathrm{~Hz}, 1 \mathrm{H}\right), 7.56-7.64(\mathrm{~m}, 2 \mathrm{H}), 7.30-7.37(\mathrm{~m}, 1 \mathrm{H})$, $7.11(\mathrm{~d}, J=2.9 \mathrm{~Hz}, 1 \mathrm{H}), 3.91-4.05(\mathrm{~m}, 1 \mathrm{H}), 2.75-2.93(\mathrm{~m}, 2 \mathrm{H}), 2.54-2.62(\mathrm{~m}, 1 \mathrm{H}), 2.47-2.55$ (m, 3 H), 2.21-2.35 (m, 3 H), 1.79-1.89 (m, 1 H), 1.65-1.79 (m, 4 H), 1.45-1.64 (m, 2 H), 1.13$1.28(\mathrm{~m}, 4 \mathrm{H}), 1.01-1.13(\mathrm{~m}, 1 \mathrm{H})$.

\section{$R a c-\left(3 R^{*}, 4 R^{*}\right)-1-C y c l o h e x y l-4-\{[5-(2,4-d i f l u o r o-p h e n y l)-i s o x a z o l e-3-c a r b o n y l]-a m i n o\}-$} piperidine-3-carboxylic acid ethyl-methyl-amide (20c). Prepared from Nethylmethylamine. LC-MS method $B: t_{R}=1.04 \mathrm{~min} ;[\mathrm{M}+\mathrm{H}]^{+}=474.91$. LC-HRMS: $\mathrm{t}_{\mathrm{R}}=0.62$ $\min ; \mathrm{m} / \mathrm{z}=474.2442$, found $=475.2526[\mathrm{M}+\mathrm{H}]^{+} .{ }^{1} \mathrm{H}$ NMR $\left(500 \mathrm{MHz}, \mathrm{D}_{6}-\mathrm{DMSO}\right) \delta: 8.69(\mathrm{~d}$, $J=8.6 \mathrm{~Hz}, 0.5 \mathrm{H}), 8.63(\mathrm{~d}, J=8.8 \mathrm{~Hz}, 0.5 \mathrm{H}), 8.01-8.09(\mathrm{~m}, 1 \mathrm{H}), 7.58\left(\mathrm{ddd}, J_{1}=11.4 \mathrm{~Hz}, J_{2}\right.$ $\left.=9.1 \mathrm{~Hz}, J_{3}=2.4 \mathrm{~Hz}, 1 \mathrm{H}\right), 7.33\left(\mathrm{td}, J_{1}=8.5 \mathrm{~Hz}, J_{2}=2.1 \mathrm{~Hz}, 1 \mathrm{H}\right), 7.08\left(\mathrm{dd}, J_{1}=6.1 \mathrm{~Hz}, J_{2}=\right.$ $2.9 \mathrm{~Hz}, 1 \mathrm{H}), 4.01-4.16(\mathrm{~m}, 1 \mathrm{H}), 3.56-3.67$ (m, $0.5 \mathrm{H}), 3.34-3.41(\mathrm{~m}, 0.5 \mathrm{H}), 3.21-3.29(\mathrm{~m}, 0.5$ H), 3.05-3.16 (m, 1.5 H), 3.01-3.05 (m, $1.5 \mathrm{H}), 2.79-2.91(\mathrm{~m}, 2 \mathrm{H}), 2.72-2.77(\mathrm{~m}, 1.5 \mathrm{H}), 2.19-$ $2.36(\mathrm{~m}, 3 \mathrm{H}), 1.59-1.85(\mathrm{~m}, 6 \mathrm{H}), 1.51-1.59(\mathrm{~m}, 1 \mathrm{H}), 1.15-1.27(\mathrm{~m}, 4 \mathrm{H}), 1.11-1.15(\mathrm{~m}, 1.5$ H), 1.01-1.11 (m, $1 \mathrm{H}), 0.88(\mathrm{t}, J=7.0 \mathrm{~Hz}, 1.5 \mathrm{H})$.

Rac-5-(2,4-Difluoro-phenyl)-isoxazole-3-carboxylic acid $\quad\left[\left(3 R^{*}, 4 R^{*}\right)-3\right.$-(azetidine-1carbonyl)-1-cyclohexyl-piperidin-4-yl]-amide (20d). Prepared from azetidine. LC-MS method $\mathrm{B}: \mathrm{t}_{\mathrm{R}}=0.93 \mathrm{~min} ;[\mathrm{M}+\mathrm{H}]^{+}=473.15 . \mathrm{LC}-\mathrm{HRMS}: \mathrm{t}_{\mathrm{R}}=0.58 \mathrm{~min} ; \mathrm{m} / \mathrm{z}=472.2286$, found $=473.2365[\mathrm{M}+\mathrm{H}]^{+} .{ }^{1} \mathrm{H}$ NMR $\left(500 \mathrm{MHz}, \mathrm{D}_{6}-\mathrm{DMSO}\right) \delta: 8.71(\mathrm{~d}, J=8.1 \mathrm{~Hz}, 1 \mathrm{H}), 8.06\left(\mathrm{td}, J_{1}\right.$ 
$\left.=6.4 \mathrm{~Hz}, J_{2}=8.5 \mathrm{~Hz}, 1 \mathrm{H}\right), 7.59\left(\mathrm{ddd}, J_{1}=2.6 \mathrm{~Hz}, J_{2}=9.3 \mathrm{~Hz}, J_{3}=11.4 \mathrm{~Hz}, 1 \mathrm{H}\right), 7.32-7.37$ (m, $1 \mathrm{H}), 7.12(\mathrm{~d}, J=2.9 \mathrm{~Hz}, 1 \mathrm{H}), 4.29-4.38(\mathrm{~m}, 1 \mathrm{H}), 4.08-4.17(\mathrm{~m}, 1 \mathrm{H}), 3.94-4.06(\mathrm{~m}, 1 \mathrm{H})$, 3.69-3.84 (m, 2 H), 2.79-2.93 (m, 2 H), 2.63-2.72 (m, $1 \mathrm{H}), 2.05-2.37$ (m, $5 \mathrm{H}), 1.78-1.86$ (m, $1 \mathrm{H}), 1.68-1.78(\mathrm{~m}, 4 \mathrm{H}), 1.52-1.63(\mathrm{~m}, 2 \mathrm{H}), 1.14-1.28(\mathrm{~m}, 4 \mathrm{H}), 1.00-1.13(\mathrm{~m}, 1 \mathrm{H})$.

Rac-5-(2,4-Difluoro-phenyl)-isoxazole-3-carboxylic acid $\quad\left[\left(3 R^{*}, 4 R^{*}\right)\right.$-1-cyclohexyl-3(pyrrolidine-1-carbonyl)-piperidin-4-yl]-amide (20e). Prepared from pyrrolidine. LC-MS method $\mathrm{B}: \mathrm{t}_{\mathrm{R}}=1.03 \mathrm{~min} ;[\mathrm{M}+\mathrm{H}]^{+}=486.92 . \mathrm{LC}-\mathrm{HRMS}: \mathrm{t}_{\mathrm{R}}=0.62 \mathrm{~min} ; \mathrm{m} / \mathrm{z}=486.2442$, found $=487.252[\mathrm{M}+\mathrm{H}]^{+} .{ }^{1} \mathrm{H}$ NMR $\left(500 \mathrm{MHz}, \mathrm{D}_{6}-\mathrm{DMSO}\right) \delta: 8.70(\mathrm{~d}, J=8.7 \mathrm{~Hz}, 1 \mathrm{H}), 8.05\left(\mathrm{td}, J_{1}\right.$ $\left.=6.5 \mathrm{~Hz}, J_{2}=8.7 \mathrm{~Hz}, 1 \mathrm{H}\right), 7.58\left(\mathrm{ddd}, J_{1}=2.5 \mathrm{~Hz}, J_{2}=9.4 \mathrm{~Hz}, J_{3}=11.4 \mathrm{~Hz}, 1 \mathrm{H}\right), 7.33\left(\mathrm{td}, J_{1}\right.$ $\left.=2.1 \mathrm{~Hz}, J_{2}=8.4 \mathrm{~Hz}, 1 \mathrm{H}\right), 7.09(\mathrm{~d}, J=2.9 \mathrm{~Hz}, 1 \mathrm{H}), 4.02-4.13(\mathrm{~m}, 1 \mathrm{H}), 3.72-3.80(\mathrm{~m}, 1 \mathrm{H})$, 3.33-3.40 (m, $1 \mathrm{H}), 3.16-3.26$ (m, $2 \mathrm{H}), 2.88-2.96$ (m, $2 \mathrm{H}), 2.80-2.86$ (m, $1 \mathrm{H}), 2.19-2.36$ (m, $3 \mathrm{H}), 1.85-1.94(\mathrm{~m}, 1 \mathrm{H}), 1.78-1.85$ (m, $2 \mathrm{H}), 1.65-1.78$ (m, $6 \mathrm{H}), 1.53-1.64(\mathrm{~m}, 2 \mathrm{H}), 1.13-1.29$ (m, $4 \mathrm{H}), 1.01-1.13(\mathrm{~m}, 1 \mathrm{H})$.

$R a c-\left(3 R^{*}, 4 R^{*}\right)-1-C y c l o h e x y l-4-\{[5-(2,4-d i f l u o r o-p h e n y l)-i s o x a z o l e-3-c a r b o n y l]-a m i n o\}-$ piperidine-3-carboxylic acid (2-methoxy-ethyl)-methyl-amide (20f). Prepared from 2methylamino-ethanol. LC-MS method $\mathrm{B}: \mathrm{t}_{\mathrm{R}}=1.05 \mathrm{~min} ;[\mathrm{M}+\mathrm{H}]^{+}=504.91$. LC-HRMS: $\mathrm{t}_{\mathrm{R}}=$ $0.62 \mathrm{~min} ; \mathrm{m} / \mathrm{z}=504.2548$, found $=505.2622[\mathrm{M}+\mathrm{H}]^{+} .{ }^{1} \mathrm{H}$ NMR $\left(500 \mathrm{MHz}, \mathrm{D}_{6}-\mathrm{DMSO}\right) \delta$ : $8.70(\mathrm{~d}, J=8.7 \mathrm{~Hz}, 0.5 \mathrm{H}), 8.45-8.52(\mathrm{~m}, 0.5 \mathrm{H}), 8.05\left(\mathrm{td}, J_{1}=6.5 \mathrm{~Hz}, J_{2}=8.6 \mathrm{~Hz}, 1 \mathrm{H}\right), 7.58$ $\left(\mathrm{ddd}, J_{1}=2.4 \mathrm{~Hz}, J_{2}=9.4 \mathrm{~Hz}, J_{3}=11.4 \mathrm{~Hz}, 1 \mathrm{H}\right), 7.33\left(\mathrm{td}, J_{1}=2.2 \mathrm{~Hz}, J_{2}=8.4 \mathrm{~Hz}, 1 \mathrm{H}\right), 7.09$ $(\mathrm{d}, J=2.9 \mathrm{~Hz}, 1 \mathrm{H}), 4.01-4.13(\mathrm{~m}, 1 \mathrm{H}), 3.85-3.96(\mathrm{~m}, 0.5 \mathrm{H}), 3.43-3.56(\mathrm{~m}, 1.5 \mathrm{H}), 3.20-3.31$ (m, 3.5 H), 3.10-3.19 (m, $1 \mathrm{H}), 3.07-3.10(\mathrm{~m}, 3 \mathrm{H}), 2.88-2.94(\mathrm{~m}, 0.5 \mathrm{H}), 2.81-2.88(\mathrm{~m}, 1.5 \mathrm{H})$, $2.77(\mathrm{~s}, 1.5 \mathrm{H}), 2.15-2.36(\mathrm{~m}, 3 \mathrm{H}), 1.78-1.84(\mathrm{~m}, 1 \mathrm{H}), 1.69-1.78(\mathrm{~m}, 4 \mathrm{H}), 1.60-1.68(\mathrm{~m}, 1 \mathrm{H})$, 1.53-1.60 (m, $1 \mathrm{H}), 1.12-1.28(\mathrm{~m}, 4 \mathrm{H}), 1.01-1.13(\mathrm{~m}, 1 \mathrm{H})$. 
$R a c-\left(3 R^{*}, 4 R^{*}\right)-1-C y c l o h e x y l-4-\{[5-(2,4-d i f l u o r o-p h e n y l)-i s o x a z o l e-3-c a r b o n y l]-a m i n o\}-$ piperidine-3-carboxylic acid (2-hydroxy-ethyl)-amide (20g). Prepared from (2-hydroxyethyl)-amine. LC-MS method $B: t_{R}=0.89 \min ;[M+H]^{+}=476.89$. LC-HRMS: $t_{R}=0.52$ min; $\mathrm{m} / \mathrm{z}=476.2235$, found $=477.2316[\mathrm{M}+\mathrm{H}]^{+} .{ }^{1} \mathrm{H}$ NMR $\left(500 \mathrm{MHz}, \mathrm{D}_{6}-\mathrm{DMSO}\right) \delta: 8.56(\mathrm{~d}, J=$ $8.5 \mathrm{~Hz}, 1 \mathrm{H}), 8.06\left(\mathrm{td}, J_{1}=8.7 \mathrm{~Hz}, J_{2}=6.5 \mathrm{~Hz}, 1 \mathrm{H}\right), 7.66(\mathrm{t}, J=5.6 \mathrm{~Hz}, 1 \mathrm{H}), 7.58\left(\mathrm{ddd}, J_{1}=\right.$ $\left.11.5 \mathrm{~Hz}, J_{2}=9.3 \mathrm{~Hz}, J_{3}=2.5 \mathrm{~Hz}, 1 \mathrm{H}\right), 7.33\left(\mathrm{td}, J_{1}=8.3 \mathrm{~Hz}, J_{2}=2.1 \mathrm{~Hz}, 1 \mathrm{H}\right), 7.11(\mathrm{~d}, J=2.9$ $\mathrm{Hz}, 1 \mathrm{H}), 4.57$ (t, $J=5.5 \mathrm{~Hz}, 1 \mathrm{H}), 3.90-4.01(\mathrm{~m}, 1 \mathrm{H}), 3.23-3.35(\mathrm{~m}, 2 \mathrm{H}), 3.00-3.12(\mathrm{~m}, 2 \mathrm{H})$, 2.83-2.91 (m, $1 \mathrm{H}), 2.77-2.83(\mathrm{~m}, 1 \mathrm{H}), 2.53-2.62(\mathrm{~m}, 1 \mathrm{H}), 2.22-2.40$ (m, $3 \mathrm{H}), 1.79-1.89$ (m, $1 \mathrm{H}), 1.67-1.79(\mathrm{~m}, 4 \mathrm{H}), 1.54-1.61(\mathrm{~m}, 1 \mathrm{H}), 1.49\left(\mathrm{ddd}, J_{1}=3.7 \mathrm{~Hz}, J_{2}=12.1 \mathrm{~Hz}, J_{3}=24.1\right.$ $\mathrm{Hz}, 1 \mathrm{H}), 1.12-1.27$ (m, $4 \mathrm{H}), 1.00-1.12(\mathrm{~m}, 1 \mathrm{H})$.

$R a c-\left(3 R^{*}, 4 R^{*}\right)-1-C y c l o h e x y l-4-\{[5-(2,4-d i f l u o r o-p h e n y l)-i s o x a z o l e-3-c a r b o n y l]-a m i n o\}-$ piperidine-3-carboxylic acid benzylamide (20h). Prepared from benzylamine. LC-MS method $\mathrm{B}: \mathrm{t}_{\mathrm{R}}=1.05 \mathrm{~min} ;[\mathrm{M}+\mathrm{H}]^{+}=523.17 . \mathrm{LC}-\mathrm{HRMS}: \mathrm{t}_{\mathrm{R}}=0.67 . \mathrm{min} ; \mathrm{m} / \mathrm{z}=522.2442$, found $=523.2515[\mathrm{M}+\mathrm{H}]^{+} .{ }^{1} \mathrm{H}$ NMR $\left(500 \mathrm{MHz}, \mathrm{D}_{6}\right.$-DMSO) $\delta: 8.64(\mathrm{~d}, J=8.6 \mathrm{~Hz}, 1 \mathrm{H}), 8.15-8.21$ $(\mathrm{m}, 1 \mathrm{H}), 8.07\left(\mathrm{td}, J_{1}=8.7 \mathrm{~Hz}, J_{2}=6.4 \mathrm{~Hz}, 1 \mathrm{H}\right), 7.60\left(\mathrm{ddd}, J_{1}=11.4 \mathrm{~Hz}, J_{2}=9.3 \mathrm{~Hz}, J_{3}=2.4\right.$ Hz, 1H), 7.32-7.38 (m, 1 H), 7.12-7.17 (m, 2 H), 7.04-7.12 (m, 4 H), 4.36-4.44 (m, 1 H), 3.984.12 (m, 2 H), 2.88-2.97 (m, 1 H), 2.80-2.88 (m, $1 \mathrm{H}), 2.67-2.75(\mathrm{~m}, 1 \mathrm{H}), 2.25-2.43(\mathrm{~m}, 3 \mathrm{H})$, 1.80-1.89 (m, $1 \mathrm{H}), 1.69-1.80$ (m, $4 \mathrm{H}), 1.49-1.63$ (m, $2 \mathrm{H}), 1.15-1.28$ (m, $4 \mathrm{H}), 1.02-1.14$ (m, $1 \mathrm{H})$.

$R a c-\left(3 R^{*}, 4 R^{*}\right)-1-C y c l o h e x y l-4-\{[5-(2,4-d i f l u o r o-p h e n y l)-i s o x a z o l e-3-c a r b o n y l]-a m i n o\}-$ piperidine-3-carboxylic acid phenylamide (20i). Prepared from aniline. LC-MS method B: $\mathrm{t}_{\mathrm{R}}=1.10 \mathrm{~min} ;[\mathrm{M}+\mathrm{H}]^{+}=509.12 . \mathrm{LC}-\mathrm{HRMS}: \mathrm{t}_{\mathrm{R}}=0.69 \mathrm{~min} ; \mathrm{m} / \mathrm{z}=508.2286$, found $=509.2355$ $[\mathrm{M}+\mathrm{H}]^{+} .{ }^{1} \mathrm{H}$ NMR $\left(500 \mathrm{MHz}, \mathrm{D}_{6}-\mathrm{DMSO}\right) \delta: 9.83(\mathrm{~s}, 1 \mathrm{H}), 8.77(\mathrm{~d}, J=8.4 \mathrm{~Hz}, 1 \mathrm{H}), 8.02(\mathrm{td}$, $\left.J_{1}=6.4 \mathrm{~Hz}, J_{2}=8.7 \mathrm{~Hz}, 1 \mathrm{H}\right), 7.54-7.60(\mathrm{~m}, 3 \mathrm{H}), 7.29-7.34(\mathrm{~m}, 1 \mathrm{H}), 7.23-7.28(\mathrm{~m}, 2 \mathrm{H}), 7.07$ $(\mathrm{d}, J=2.9 \mathrm{~Hz}, 1 \mathrm{H}), 6.97-7.03(\mathrm{~m}, 1 \mathrm{H}), 4.06-4.16(\mathrm{~m}, 1 \mathrm{H}), 3.02-3.09$ (m, $1 \mathrm{H}), 2.81-2.91(\mathrm{~m}$, 
$2 \mathrm{H}), 2.28-2.42(\mathrm{~m}, 3 \mathrm{H}), 1.84-1.93(\mathrm{~m}, 1 \mathrm{H}), 1.69-1.81(\mathrm{~m}, 4 \mathrm{H}), 1.51-1.62$ (m, $2 \mathrm{H}), 1.15-1.30$ (m, $4 \mathrm{H}), 1.02-1.14(\mathrm{~m}, 1 \mathrm{H})$.

$R a c-\left(3 R^{*}, 4 R^{*}\right)-1-C y c l o h e x y l-4-\{[5-(2,4-d i f l u o r o-p h e n y l)-i s o x a z o l e-3-c a r b o n y l]-a m i n o\}-$ piperidine-3-carboxylic acid (pyridin-2-ylmethyl)-amide (20j). Prepared from pyridin-2ylmethanamine. LC-MS method $\mathrm{B}: \mathrm{t}_{\mathrm{R}}=0.98 \mathrm{~min} ;[\mathrm{M}+\mathrm{H}]^{+}=523.86$. LC-HRMS: $\mathrm{t}_{\mathrm{R}}=0.54$ $\min ; \mathrm{m} / \mathrm{z}=523.2395$, found $=524.2479[\mathrm{M}+\mathrm{H}]^{+} .1 \mathrm{H} \mathrm{M}-\mathrm{NMR}(400 \mathrm{MHz}, \mathrm{DMSO}$, water suppression) $\delta:$ 8.69-8.76 (m, $1 \mathrm{H}), 8.35-8.41(\mathrm{~m}, 1 \mathrm{H}), 8.30-8.35(\mathrm{~m}, 1 \mathrm{H}), 8.03-8.11(\mathrm{~m}, 1 \mathrm{H})$, 7.55-7.64 (m, $1 \mathrm{H})$, 7.38-7.45 (m, $1 \mathrm{H}), 7.31-7.38$ (m, $1 \mathrm{H})$, 7.15-7.22 (m, $1 \mathrm{H})$, 7.08-7.13 (m, $2 \mathrm{H}), 4.40-4.49$ (m, $1 \mathrm{H}), 4.16-4.23$ (m, $1 \mathrm{H}), 3.96-4.08$ (m, $1 \mathrm{H}), 2.91-2.98$ (m, $1 \mathrm{H}), 2.79-2.86$ (m, $1 \mathrm{H}), 2.67-2.79$ (m, $1 \mathrm{H}), 2.25-2.43$ (m, $3 \mathrm{H}), 1.80-1.89$ (m, $1 \mathrm{H}), 1.69-1.80$ (m, $4 \mathrm{H}), 1.50$ 1.62 (m, 2 H), 1.14-1.28 (m, 4 H), 1.02-1.14 (m, 1 H).

$R a c-\left(3 R^{*}, 4 R^{*}\right)-1-C y c l o h e x y l-4-\{[5-(2,4-d i f l u o r o-p h e n y l)-i s o x a z o l e-3-c a r b o n y l]-a m i n o\}-$ piperidine-3-carboxylic acid phenethyl-amide (20k). Prepared from phenethylamine. LCMS method B: $t_{R}=1.13 \mathrm{~min} ;[\mathrm{M}+\mathrm{H}]^{+}=537.16$. LC-HRMS: $\mathrm{t}_{\mathrm{R}}=0.71 \mathrm{~min} ; \mathrm{m} / \mathrm{z}=536.2599$, found $=537.2678[\mathrm{M}+\mathrm{H}]^{+} .{ }^{1} \mathrm{H}$ NMR $\left(500 \mathrm{MHz}, \mathrm{D}_{6}\right.$-DMSO) $\delta: 8.55(\mathrm{~d}, J=8.4 \mathrm{~Hz}, 1 \mathrm{H}), 8.05$ $\left(\mathrm{td}, J_{1}=8.7 \mathrm{~Hz}, J_{2}=6.4 \mathrm{~Hz}, 1 \mathrm{H}\right), 7.77(\mathrm{t}, J=5.5 \mathrm{~Hz}, 1 \mathrm{H}), 7.58\left(\mathrm{ddd}, J_{1}=2.6 \mathrm{~Hz}, J_{2}=9.3 \mathrm{~Hz}\right.$, $\left.J_{3}=11.6 \mathrm{~Hz}, 1 \mathrm{H}\right), 7.30-7.36(\mathrm{~m}, 1 \mathrm{H}), 7.18-7.24(\mathrm{~m}, 2 \mathrm{H}), 7.10-7.16(\mathrm{~m}, 4 \mathrm{H}), 3.92-4.03(\mathrm{~m}, 1$ H), 3.17-3.31 (m, 2 H), 2.74-2.84 (m, 2 H), 2.56-2.70 (m, 3 H), 2.20-2.33 (m, 3 H), 1.79-1.87 (m, 1 H), 1.66-1.79 (m, 4 H), 1.45-1.63 (m, 2 H), 1.13-1.28 (m, 4 H), 1.02-1.13 (m, $1 \mathrm{H})$.

$R a c-\left(3 R^{*}, 4 R^{*}\right)-1-C y c l o h e x y l-4-\{[5-(2,4-d i f l u o r o-p h e n y l)-i s o x a z o l e-3-c a r b o n y l]-a m i n o\}-$ piperidine-3-carboxylic acid methyl-phenethyl-amide (201). Prepared from N-methyl-2phenylethylamine. LC-MS method $\mathrm{B}: \mathrm{t}_{\mathrm{R}}=1.18 \mathrm{~min} ;[\mathrm{M}+\mathrm{H}]^{+}=551.17$. LC-HRMS: $\mathrm{t}_{\mathrm{R}}=0.75$ $\min ; \mathrm{m} / \mathrm{z}=550.2755$, found $=551.2838[\mathrm{M}+\mathrm{H}]^{+} .{ }^{1} \mathrm{H}$ NMR $\left(500 \mathrm{MHz}, \mathrm{D}_{6}-\mathrm{DMSO}\right) \delta: 8.69(\mathrm{~d}$, $J=8.2 \mathrm{~Hz}, 1 \mathrm{H}, 1 \mathrm{H}), 8.04\left(\mathrm{td}, J_{1}=8.5 \mathrm{~Hz}, J_{2}=6.6 \mathrm{~Hz}, 1 \mathrm{H}\right), 7.57\left(\mathrm{~m}, J_{1}=2.1 \mathrm{~Hz}, J_{2}=9.8 \mathrm{~Hz}\right.$, 
$1 \mathrm{H})$, 7.30-7.36 (m, $2 \mathrm{H}), 7.19-7.29(\mathrm{~m}, 2 \mathrm{H}), 7.12-7.18(\mathrm{~m}, 2 \mathrm{H}), 7.07-7.12(\mathrm{~m}, 1 \mathrm{H}), 4.01-4.14$ (m, $1 \mathrm{H}), 3.87-4.00(\mathrm{~m}, 0.5 \mathrm{H}), 3.51-3.63(\mathrm{~m}, 0.5 \mathrm{H}), 3.29-3.45(\mathrm{~m}, 1 \mathrm{H}), 3.00-3.13(\mathrm{~m}, 1 \mathrm{H})$, 2.94-3.00 (m, 1.5 H), 2.86-2.94 (m, 0.5 H), 2.76-2.86 (m, 1.5 H), 2.72-2.76 (m, $1.5 \mathrm{H}), 2.60-$ $2.72(\mathrm{~m}, 2 \mathrm{H}), 2.15-2.35(\mathrm{~m}, 2.5 \mathrm{H}), 2.03-2.15(\mathrm{~m}, 0.5 \mathrm{H}), 1.50-1.87$ (m, $7 \mathrm{H}), 0.99-1.28$ (m, 5 $\mathrm{H})$.

Stereoisomeric mixture $\left(3 R^{*}, 4 R^{*}\right)-1-C y c l o h e x y l-4-\{[5-(2,4-d i f l u o r o-p h e n y l)-i s o x a z o l e-3-$ carbonyl]-amino\}-piperidine-3-carboxylic acid (( $\left.R^{*}\right)$-1-pyridin-2-yl-ethyl)-amide (20m). Prepared from 1-(2-pyridyl)ethylamine. QC LC-MS: $t_{R}=0.67 \mathrm{~min} ;[\mathrm{M}+\mathrm{H}]^{+}=538.20$ and $\mathrm{t}_{\mathrm{R}}$ $=0.68 \mathrm{~min} ;[\mathrm{M}+\mathrm{H}]^{+}=538.20 . \mathrm{LC}-\mathrm{HRMS}: \mathrm{t}_{\mathrm{R}}=0.58 \mathrm{~min} ; \mathrm{m} / \mathrm{z}=537.2551$, found $=538.2631$ $[\mathrm{M}+\mathrm{H}]^{+}$.

\section{$(3 R, 4 R)-1-C y c l o h e x y l-4-\{[5-(2,4-d i f l u o r o-p h e n y l)-i s o x a z o l e-3-c a r b o n y l]-a m i n o\}-$} piperidine-3-carboxylic acid ((R)-1-pyridin-2-yl-ethyl)-amide (20n) was synthesised by HATU amidation of $(3 R, 4 R)-1$-cyclohexyl-4-\{[5-(2,4-difluoro-phenyl)-isoxazole-3carbonyl]-amino -piperidine-3-carboxylic acid with (R)-1-(pyridin-2-yl)ethanamine. LC-MS method $\mathrm{A}: \mathrm{t}_{\mathrm{R}}=0.63 \mathrm{~min} ;[\mathrm{M}+\mathrm{H}]^{+}=538.33$. Chiral HPLC: $\mathrm{t}_{\mathrm{R}}=11.3 \mathrm{~min} ;>99 \%$ ee; column: ChiralPak IB 4.6x250 mm, $5 \mu \mathrm{M}$; detector wavelength: $254 \mathrm{~nm}$; eluent: $90 \%$ heptane $0.05 \%$ DEA; $10 \%$ ethanol $0.05 \%$ DEA; flow: $0.8 \mathrm{~mL} / \mathrm{min}$; BPR: 150 bar; temperature: $25^{\circ} \mathrm{C}$; injection volume: $4 \mu$ l. LC-HRMS: $\mathrm{t}_{\mathrm{R}}=0.58 \mathrm{~min} ; \mathrm{m} / \mathrm{z}=537.2551$, found $=538.2631[\mathrm{M}+\mathrm{H}]^{+} .{ }^{1} \mathrm{H} \mathrm{NMR}$ $\left(400 \mathrm{MHz}, \mathrm{D}_{6}-\mathrm{DMSO}\right) \delta: 8.69(\mathrm{~m}, 1 \mathrm{H}), 8.33(\mathrm{~d}, J=4.7 \mathrm{~Hz}, 1 \mathrm{H}), 8.22(\mathrm{~d}, J=7.6 \mathrm{~Hz}, 1 \mathrm{H})$, $8.08(\mathrm{~m}, 1 \mathrm{H}), 7.60(\mathrm{~m}, 1 \mathrm{H}), 7.35\left(\mathrm{td}, J_{1}=8.5 \mathrm{~Hz}, J_{2}=2.3 \mathrm{~Hz}, 1 \mathrm{H}\right), 7.24(\mathrm{~m}, 2 \mathrm{H}), 7.06(\mathrm{~d}, J$ $=2.8 \mathrm{~Hz}, 1 \mathrm{H}), 7.00(\mathrm{~m}, 1 \mathrm{H}), 4.87$ (quint, $J=7.0 \mathrm{~Hz}, 1 \mathrm{H}), 3.90-3.99(\mathrm{~m}, 1 \mathrm{H}), 2.93(\mathrm{~d}, J=$ $9.3 \mathrm{~Hz}, 1 \mathrm{H}), 2.81(\mathrm{~d}, J=11.3 \mathrm{~Hz}, 1 \mathrm{H}), 2.75\left(\mathrm{td}, J_{1}=3.5 \mathrm{~Hz}, J_{2}=11.0 \mathrm{~Hz}, 1 \mathrm{H}\right), 2.38(\mathrm{~m}, 1$ H), 2.26-2.34 (m, 2 H), 1.80-1.84 (m, $1 \mathrm{H}), 1.74(\mathrm{~d}, J=8.0 \mathrm{~Hz}, 4 \mathrm{H}), 1.49-1.60$ (m, $2 \mathrm{H}), 1.34$ $(\mathrm{d}, J=7.0 \mathrm{~Hz}, 3 \mathrm{H}), 1.16-1.26(\mathrm{~m}, 5 \mathrm{H})$. 
(3S,4S)-1-Cyclohexyl-4-\{[5-(2,4-difluoro-phenyl)-isoxazole-3-carbonyl]-amino\}-

piperidine-3-carboxylic acid ((R)-1-pyridin-2-yl-ethyl)-amide (200) was synthesised by HATU amidation of $(3 S, 4 S)-1-c y c l o h e x y l-4-\{[5-(2,4-d i f l u o r o-p h e n y l)-i s o x a z o l e-3-c a r b o n y l]-$ amino -piperidine-3-carboxylic acid 19 with $(R)$-1-(pyridin-2-yl)ethanamine. LC-MS method $\mathrm{A}: \mathrm{t}_{\mathrm{R}}=0.64 \mathrm{~min} ;[\mathrm{M}+\mathrm{H}]^{+}=538.21$. Chiral HPLC: $\mathrm{t}_{\mathrm{R}}=15.8 \mathrm{~min} ;>99 \%$ ee; column: ChiralPak IB $4.6 \times 250 \mathrm{~mm}, 5 \mu \mathrm{M}$; detector wavelength: $254 \mathrm{~nm}$; eluent: $90 \%$ heptane $0.05 \%$ DEA; $10 \%$ ethanol 0.05\% DEA; flow: $0.8 \mathrm{~mL} / \mathrm{min}$; $\mathrm{BPR}: 150$ bar; temperature: $25^{\circ} \mathrm{C}$; injection volume: $5 \mu$ l. LC-HRMS: $\mathrm{t}_{\mathrm{R}}=0.59 \mathrm{~min} ; \mathrm{m} / \mathrm{z}=537.2551$, found $=538.2633[\mathrm{M}+\mathrm{H}]^{+} .{ }^{1} \mathrm{H} \mathrm{NMR}(400$ MHz, D6-DMSO) $\delta: 8.71(\mathrm{~d}, J=8.6 \mathrm{~Hz}, 1 \mathrm{H}), 8.50-8.51(\mathrm{~m}, 1 \mathrm{H}), 8.16(\mathrm{~d}, J=8.1 \mathrm{~Hz}, 1 \mathrm{H})$, $8.06\left(\mathrm{td}, J_{1}=8.7 \mathrm{~Hz}, J_{2}=6.4 \mathrm{~Hz}, 1 \mathrm{H}\right), 7.72\left(\mathrm{td}, J_{1}=7.7 \mathrm{~Hz}, J_{2}=1.8 \mathrm{~Hz}, 1 \mathrm{H}\right), 7.58\left(\mathrm{ddd}, J_{1}=\right.$ $\left.11.5 \mathrm{~Hz}, J_{2}=9.3 \mathrm{~Hz}, J_{3}=2.5 \mathrm{~Hz}, 1 \mathrm{H}\right), 7.31-7.35(\mathrm{~m}, 2 \mathrm{H}), 7.25\left(\mathrm{ddd}, J_{1}=7.5 \mathrm{~Hz}, J_{2}=4.8 \mathrm{~Hz}\right.$, $\left.J_{3}=1.0 \mathrm{~Hz}, 1 \mathrm{H}\right), 7.15(\mathrm{~d}, J=2.9 \mathrm{~Hz}, 1 \mathrm{H}), 4.92(\mathrm{~m}, 1 \mathrm{H}), 4.02(\mathrm{~m}, 1 \mathrm{H}), 2.90(\mathrm{~m}, 2 \mathrm{H}), 2.70-$ $2.74(\mathrm{~m}, 1 \mathrm{H}), 2.37-2.47$ (m, $3 \mathrm{H}), 1.87-1.90$ (m, $1 \mathrm{H}), 1.73-1.74(\mathrm{~m}, 4 \mathrm{H}), 1.49-1.58(\mathrm{~m}, 2 \mathrm{H})$, 1.17-1.22 (m, $7 \mathrm{H}), 1.02-1.09(\mathrm{~m}, 1 \mathrm{H})$.

\section{(3R,4R)-1-Cyclohexyl-4-\{[5-(2,4-difluoro-phenyl)-isoxazole-3-carbonyl]-amino\}-}

piperidine-3-carboxylic acid ((S)-1-pyridin-2-yl-ethyl)-amide (20p) was synthesised by HATU amidation of $(3 R, 4 R)-1$-cyclohexyl-4-\{[5-(2,4-difluoro-phenyl)-isoxazole-3carbonyl]-amino - -piperidine-3-carboxylic acid with (S)-1-(pyridin-2-yl)ethanamine. LC-MS method $A: t_{R}=0.65 \mathrm{~min} ;[\mathrm{M}+\mathrm{H}]^{+}=538.23$. Chiral HPLC: $\mathrm{t}_{\mathrm{R}}=13.6 \mathrm{~min} ; 96.5 \%$ ee; column: ChiralPak IB 4.6x250 mm, $5 \mu \mathrm{M}$; detector wavelength: $260 \mathrm{~nm}$; eluent: $90 \%$ heptane $0.05 \%$ DEA; $10 \%$ ethanol $0.05 \%$ DEA; flow: $0.8 \mathrm{~mL} / \mathrm{min}$; BPR: 150 bar; temperature: $25^{\circ} \mathrm{C}$; injection volume: $2.5 \mu \mathrm{l}$. LC-HRMS: $\mathrm{t}_{\mathrm{R}}=0.59 \mathrm{~min} ; \mathrm{m} / \mathrm{z}=537.2551$, found $=538.2628[\mathrm{M}+\mathrm{H}]^{+} .{ }^{1} \mathrm{H}$ NMR (400 MHz, D6-DMSO) $\delta: 8.68(\mathrm{~d}, J=8.7 \mathrm{~Hz}, 1 \mathrm{H}), 8.52(\mathrm{~d}, J=4.2 \mathrm{~Hz}, 1 \mathrm{H}), 8.13(\mathrm{~d}, J$ $=8.1 \mathrm{~Hz}, 1 \mathrm{H}), 8.07(\mathrm{q}, J=8.6 \mathrm{~Hz}, 1 \mathrm{H}), 7.73(\mathrm{t}, J=7.3 \mathrm{~Hz}, 1 \mathrm{H}), 7.58(\mathrm{t}, J=9.3 \mathrm{~Hz}, 1 \mathrm{H})$, $7.33(\mathrm{~d}, J=8.0 \mathrm{~Hz}, 2 \mathrm{H}), 7.26(\mathrm{t}, J=5.6 \mathrm{~Hz}, 1 \mathrm{H}), 7.14(\mathrm{~d}, J=2.4 \mathrm{~Hz}, 1 \mathrm{H}), 4.93(\mathrm{t}, J=7.8$ 
Hz, $1 \mathrm{H}), 3.96-4.03$ (m, $1 \mathrm{H}), 2.80-2.89$ (m, $2 \mathrm{H}), 2.65-2.72$ (m, $1 \mathrm{H}), 2.28-2.42$ (m, $3 \mathrm{H}), 1.86-$ $1.91(\mathrm{~m}, 1 \mathrm{H}), 1.69-1.78(\mathrm{~m}, 5 \mathrm{H}), 1.55-1.60$ (m, $1 \mathrm{H}), 1.49-1.53$ (m, $1 \mathrm{H}), 1.19-1.23(\mathrm{~m}, 6 \mathrm{H})$, $1.04-1.10(\mathrm{~m}, 1 \mathrm{H})$.

(3S,4S)-1-Cyclohexyl-4-\{[5-(2,4-difluoro-phenyl)-isoxazole-3-carbonyl]-amino\}piperidine-3-carboxylic acid ((S)-1-pyridin-2-yl-ethyl)-amide (20q) was synthesised by HATU amidation of (3S,4S)-1-cyclohexyl-4-\{[5-(2,4-difluoro-phenyl)-isoxazole-3-carbonyl]amino -piperidine-3-carboxylic acid 19 with $(S)$-1-(pyridin-2-yl)ethanamine. LC-MS method $\mathrm{A}: \mathrm{t}_{\mathrm{R}}=0.67 \mathrm{~min} ;[\mathrm{M}+\mathrm{H}]^{+}=538.28$. Chiral HPLC: $\mathrm{t}_{\mathrm{R}}=17.3 \mathrm{~min} ; 96 \%$ ee; column: ChiralPak IB 4.6x250 mm, $5 \mu \mathrm{M}$; detector wavelength: $260 \mathrm{~nm}$; eluent: $90 \%$ heptane $0.05 \%$ DEA; $10 \%$ ethanol $0.05 \%$ DEA; flow: $0.8 \mathrm{~mL} / \mathrm{min}$; BPR: 150 bar; temperature: $25^{\circ} \mathrm{C}$; injection volume: $3 \mu$ l. LC-HRMS: $\mathrm{t}_{\mathrm{R}}=0.58 \mathrm{~min} ; \mathrm{m} / \mathrm{z}=537.2551$, found $=538.2631[\mathrm{M}+\mathrm{H}]^{+} .{ }^{1} \mathrm{H}$ NMR $(400$ MHz, D 6 -DMSO) $\delta: 8.63(\mathrm{~d}, J=8.6 \mathrm{~Hz}, 1 \mathrm{H}), 8.33(\mathrm{~d}, J=4.6 \mathrm{~Hz}, 1 \mathrm{H}), 8.15(\mathrm{~d}, J=7.7 \mathrm{~Hz}, 1$ $\mathrm{H}), 8.08\left(\mathrm{td}, J_{1}=8.7 \mathrm{~Hz}, J_{2}=6.6 \mathrm{~Hz}, 1 \mathrm{H}\right), 7.60(\mathrm{~m}, 1 \mathrm{H}), 7.35\left(\mathrm{td}, J_{1}=8.5 \mathrm{~Hz}, J_{2}=2.2 \mathrm{~Hz}, 1\right.$ H), 7.22-7.29 (m, $2 \mathrm{H}), 7.03(\mathrm{~d}, J=2.8 \mathrm{~Hz}, 1 \mathrm{H}), 7.00(\mathrm{~m}, 1 \mathrm{H}), 4.88$ (quint, $J=7.3 \mathrm{~Hz}, 1 \mathrm{H}$ ), $3.94(\mathrm{~m}, 1 \mathrm{H}), 2.93(\mathrm{~d}, J=9.2 \mathrm{~Hz}, 1 \mathrm{H}), 2.81(\mathrm{~d}, J=11.1 \mathrm{~Hz}, 1 \mathrm{H}), 2.74\left(\mathrm{td}, J_{1}=10.8 \mathrm{~Hz}, J_{2}=\right.$ $3.4 \mathrm{~Hz}, 1 \mathrm{H}), 2.38(\mathrm{~m}, 1 \mathrm{H}), 2.26-2.34(\mathrm{~m}, 2 \mathrm{H}), 1.82\left(\mathrm{dd}, J_{1}=12.5 \mathrm{~Hz}, J_{2}=3.9 \mathrm{~Hz}, 1 \mathrm{H}\right), 1.74$ $(\mathrm{d}, J=7.5 \mathrm{~Hz}, 4 \mathrm{H}), 1.48-1.59(\mathrm{~m}, 2 \mathrm{H}), 1.35(\mathrm{~d}, J=7.0 \mathrm{~Hz}, 3 \mathrm{H}), 1.21(\mathrm{~m}, 4 \mathrm{H}), 1.06-1.11(\mathrm{~m}$, $1 \mathrm{H})$.

\section{(3S,4S)-1-Cyclohexyl-4-\{[5-(2,4-difluoro-phenyl)-isoxazole-3-carbonyl]-amino\}-}

piperidine-3-carboxylic acid benzylamide (21a) was synthesised by HATU amidation of the chiral acid 19 with benzyl amine. LC-MS method A: $t_{R}=0.76 \mathrm{~min} ;[\mathrm{M}+\mathrm{H}]^{+}=523.20$. LCHRMS: $\mathrm{t}_{\mathrm{R}}=0.68 \mathrm{~min} ; \mathrm{m} / \mathrm{z}=522.2442$, found $=523.2525[\mathrm{M}+\mathrm{H}]^{+} .{ }^{1} \mathrm{H}$ NMR $\left(400 \mathrm{MHz}, \mathrm{D}_{6}-\right.$ DMSO) $\delta: 8.66(\mathrm{~d}, J=8.5 \mathrm{~Hz}, 1 \mathrm{H}), 8.19(\mathrm{t}, J=5.8 \mathrm{~Hz}, 1 \mathrm{H}), 8.07\left(\mathrm{td}, J_{1}=8.7 \mathrm{~Hz}, J_{2}=6.5\right.$ $\mathrm{Hz}, 1 \mathrm{H}), 7.57-7.63(\mathrm{~m}, 1 \mathrm{H}), 7.35\left(\mathrm{td}, J_{1}=8.3 \mathrm{~Hz}, J_{2}=2.1 \mathrm{~Hz}, 1 \mathrm{H}\right), 7.13-7.16(\mathrm{~m}, 2 \mathrm{H}), 7.11$ 
$(\mathrm{d}, J=2.9 \mathrm{~Hz}, 1 \mathrm{H}), 7.07\left(\mathrm{dd}, J_{1}=5.1 \mathrm{~Hz}, J_{2}=2.0 \mathrm{~Hz}, 3 \mathrm{H}\right), 4.40(\mathrm{~m}, 1 \mathrm{H}), 4.07\left(\mathrm{dd}, J_{1}=15.4\right.$ $\left.\mathrm{Hz}, J_{2}=5.1 \mathrm{~Hz}, 2 \mathrm{H}\right), 2.92(\mathrm{~d}, J=10.0 \mathrm{~Hz}, 1 \mathrm{H}), 2.83(\mathrm{~d}, J=9.3 \mathrm{~Hz}, 1 \mathrm{H}), 2.68-2.73(\mathrm{~m}, 1 \mathrm{H})$, 2.27-2.43 (m, $3 \mathrm{H}), 1.84(\mathrm{~d}, J=10.3 \mathrm{~Hz}, 1 \mathrm{H}), 1.74(\mathrm{~d}, J=6.5 \mathrm{~Hz}, 4 \mathrm{H}), 1.51-1.59(\mathrm{~m}, 2 \mathrm{H})$, 1.15-1.23 (m, $5 \mathrm{H}), 1.03-1.11(\mathrm{~m}, 1 \mathrm{H})$.

(3S,4S)-1-Cyclohexyl-4-\{[5-(2,4-difluoro-phenyl)-isoxazole-3-carbonyl]-amino\}piperidine-3-carboxylic acid ((R)-1-phenyl-ethyl)-amide (21b) was synthesised by HATU amidation of the chiral acid 19 with $(R)-(+)-\alpha-m e t h y l b e n z y l$ amine. QC LC-MS: $t_{R}=0.81$ $\min ;[\mathrm{M}+\mathrm{H}]^{+}=537.50$. LC-HRMS: $\mathrm{t}_{\mathrm{R}}=0.73 \mathrm{~min} ; \mathrm{m} / \mathrm{z}=536.2598$, found $=537.2678[\mathrm{M}+$ $\mathrm{H}]^{+} .{ }^{1} \mathrm{H}$ NMR (400 MHz, CDCl3) $\delta: 7.96$ (q, $\left.J=8.3 \mathrm{~Hz}, 1 \mathrm{H}\right), 7.28$ (s, $\left.3 \mathrm{H}\right), 7.20-7.24$ (m, 2 H), 6.99-7.09 (m, 4 H), $5.12(\mathrm{~m}, 1 \mathrm{H}), 4.23-4.31$ (m, $1 \mathrm{H}), 3.00-3.05$ (m, $1 \mathrm{H}), 2.83-2.87$ (m, 1 H), 2.67-2.73 (m, 2 H), 2.56 (m, 1H), 2.35-2.45 (m, $1 \mathrm{H}), 2.01-2.07$ (m, $1 \mathrm{H}), 1.79-1.88$ (m, 7 H), 1.64-1.67 (m, $1 \mathrm{H}), 1.43(\mathrm{~d}, J=7.0 \mathrm{~Hz}, 3 \mathrm{H}), 1.26(\mathrm{~m}, J=10.1 \mathrm{~Hz}, 4 \mathrm{H})$.

(3S,4S)-1-Cyclohexyl-4-\{[5-(2,4-difluoro-phenyl)-isoxazole-3-carbonyl]-amino\}piperidine-3-carboxylic acid ((S)-2-hydroxy-1-phenyl-ethyl)-amide (21c) was synthesised by HATU amidation of the chiral acid 19 with (S)-2-amino-2-phenyl-ethanol. LC-MS method $\mathrm{B}: \mathrm{t}_{\mathrm{R}}=0.98 \mathrm{~min} ;[\mathrm{M}+\mathrm{H}]^{+}=553.21$. LC-HRMS: $\mathrm{t}_{\mathrm{R}}=0.64 \mathrm{~min} ; \mathrm{m} / \mathrm{z}=552.2548$, found $=$ $553.2634[\mathrm{M}+\mathrm{H}]^{+} .{ }^{1} \mathrm{H}$ NMR (400 MHz, D $\left.-\mathrm{DMSO}\right) \delta: 8.63(\mathrm{~d}, J=8.8 \mathrm{~Hz}, 1 \mathrm{H}), 8.06$ (m, 2 H), $7.58(\mathrm{t}, J=10.3 \mathrm{~Hz}, 1 \mathrm{H}), 7.31-7.35(\mathrm{~m}, 1 \mathrm{H}), 7.27(\mathrm{~m}, 5 \mathrm{H}), 7.19-7.22(\mathrm{~m}, 1 \mathrm{H}), 7.14(\mathrm{~d}$, $J=2.2 \mathrm{~Hz}, 1 \mathrm{H}), 4.70-4.77$ (m, $2 \mathrm{H}), 3.99-4.04$ (m, $1 \mathrm{H}), 2.79-2.90$ (m, $1 \mathrm{H}), 2.67-2.73$ (m, 1 H), 2.26-2.38 (m, 2 H), 1.84-1.90 (m, 1 H), 1.67-1.77 (m, 5 H), 1.45-1.59 (m, 2 H), 1.13-1.26 (m, $6 \mathrm{H}), 1.02-1.09(\mathrm{~m}, 1 \mathrm{H})$.

$(3 S, 4 S)-1-C y c l o h e x y l-4-\{[5-(2,4-d i f l u o r o-p h e n y l)-i s o x a z o l e-3-c a r b o n y l]-a m i n o\}-$ piperidine-3-carboxylic acid (2-pyridin-2-yl-ethyl)-amide (21d) was synthesised by HATU amidation of the chiral acid 19 with 2-(pyridin-2-yl)ethan-1-amine. LC-MS method B: $t_{R}=$ $0.99 \mathrm{~min} ;[\mathrm{M}+\mathrm{H}]^{+}=538.22$. LC-HRMS: $\mathrm{t}_{\mathrm{R}}=0.47 \mathrm{~min} ; \mathrm{m} / \mathrm{z}=537.2551$, found $=538.2631[\mathrm{M}$ 
$+\mathrm{H}] .{ }^{1} \mathrm{H}$ NMR (400 MHz, D 6 -DMSO) $\delta: 8.57(\mathrm{~d}, J=8.5 \mathrm{~Hz}, 1 \mathrm{H}), 8.44(\mathrm{~d}, J=3.8 \mathrm{~Hz}, 1 \mathrm{H})$, 8.05 (m, $1 \mathrm{H}), 7.78-7.81(\mathrm{~m}, 1 \mathrm{H}), 7.55-7.61(\mathrm{~m}, 2 \mathrm{H}), 7.31-7.35(\mathrm{~m}, 1 \mathrm{H}), 7.15(\mathrm{~m}, 2 \mathrm{H}), 7.11$ $(\mathrm{d}, J=2.8 \mathrm{~Hz}, 1 \mathrm{H}), 3.92-4.02(\mathrm{~m}, 1 \mathrm{H}), 2.74-2.84(\mathrm{~m}, 5 \mathrm{H}), 2.23-2.29(\mathrm{~m}, 3 \mathrm{H}), 1.80-1.83(\mathrm{~m}$, $1 \mathrm{H}), 1.70-1.73(\mathrm{~m}, 5 \mathrm{H}), 1.55-1.59(\mathrm{~m}, 2 \mathrm{H}), 1.47-1.52(\mathrm{~m}, 1 \mathrm{H}), 1.15-1.22(\mathrm{~m}, 4 \mathrm{H}), 1.06-1.12$ (m, $1 \mathrm{H})$.

(3S,4S)-1-Cyclohexyl-4-\{[5-(2,4-difluoro-phenyl)-isoxazole-3-carbonyl]-amino\}piperidine-3-carboxylic acid (1-methyl-1-pyridin-2-yl-ethyl)-amide (21e) was synthesised by HATU amidation of the chiral acid 19 with 2-(pyridin-2-yl)propan-2-amine. LC-MS method $A: t_{R}=0.68 \min ;[M+H]^{+}=552.23$. LC-HRMS: $t_{R}=0.61 \mathrm{~min} ; \mathrm{m} / \mathrm{z}=551.2707$, found $=552.2778[\mathrm{M}+\mathrm{H}] .{ }^{1} \mathrm{H}$ NMR $\left(400 \mathrm{MHz}, \mathrm{D}_{6}-\mathrm{DMSO}\right) \delta: 8.74(\mathrm{~d}, J=8.5 \mathrm{~Hz}, 1 \mathrm{H}), 8.39(\mathrm{~d}, J=$ 4.6 Hz, $1 \mathrm{H}), 8.09(\mathrm{~m}, 1 \mathrm{H}), 8.00(\mathrm{~s}, 1 \mathrm{H}), 7.57-7.63(\mathrm{~m}, 1 \mathrm{H}), 7.31-7.39(\mathrm{~m}, 3 \mathrm{H}), 7.16(\mathrm{~d}, J=$ $2.7 \mathrm{~Hz}, 1 \mathrm{H}), 7.09(\mathrm{~m}, 1 \mathrm{H}), 3.87-3.96(\mathrm{~m}, 1 \mathrm{H}), 2.91(\mathrm{~d}, J=9.3 \mathrm{~Hz}, 1 \mathrm{H}), 2.81(\mathrm{~d}, J=11.0 \mathrm{~Hz}$ $1 \mathrm{H}), 2.68-2.73$ (m, $2 \mathrm{H}), 2.24-2.37$ (m, $4 \mathrm{H}), 1.83-1.87$ (m, $1 \mathrm{H}), 1.69-1.75$ (m, $4 \mathrm{H}), 1.55-1.60$ (m, $1 \mathrm{H}), 1.52(\mathrm{~s}, 3 \mathrm{H}), 1.44(\mathrm{~s}, 3 \mathrm{H}), 1.21(\mathrm{~s}, 4 \mathrm{H})$.

(3S,4S)-1-Cyclohexyl-4-\{[5-(2,4-difluoro-phenyl)-isoxazole-3-carbonyl]-amino\}piperidine-3-carboxylic acid (1-pyridin-2-yl-cyclopropyl)-amide (21f) was synthesised by HATU amidation of the chiral acid 19 with 1-(pyridin-2-yl)cyclopropan-1-amine. LC-MS method B: $\mathrm{t}_{\mathrm{R}}=0.98 \mathrm{~min} ;[\mathrm{M}+\mathrm{H}]+=550.22 . \mathrm{LC}-\mathrm{HRMS}: \mathrm{t}_{\mathrm{R}}=0.59 \mathrm{~min} ; \mathrm{m} / \mathrm{z}=549.2551$, found $=550.2618[\mathrm{M}+\mathrm{H}] .{ }^{1} \mathrm{H}$ NMR $\left(400 \mathrm{MHz}, \mathrm{D}_{6}\right.$-DMSO) $\delta: 8.80(\mathrm{~d}, J=8.7 \mathrm{~Hz}, 1 \mathrm{H}), 8.48(\mathrm{~m}, 1$ H), $8.35(\mathrm{~d}, J=4.6 \mathrm{~Hz}, 1 \mathrm{H}), 8.10(\mathrm{~m}, 1 \mathrm{H}), 7.58-7.63(\mathrm{~m}, 1 \mathrm{H}), 7.33-7.37(\mathrm{~m}, 1 \mathrm{H}), 7.30(\mathrm{~s}, 2$ H), $7.18(\mathrm{~d}, J=2.7 \mathrm{~Hz}, 1 \mathrm{H}), 7.00-7.06(\mathrm{~m}, 1 \mathrm{H}), 3.98-4.08(\mathrm{~m}, 1 \mathrm{H}), 2.95(\mathrm{~d}, J=9.8 \mathrm{~Hz}, 1 \mathrm{H})$, $2.84(\mathrm{~d}, J=10.8 \mathrm{~Hz}, 1 \mathrm{H}), 2.68-2.75(\mathrm{~m}, 1 \mathrm{H}), 2.27-2.42(\mathrm{~m}, 3 \mathrm{H}), 1.85(\mathrm{~d}, J=11.0 \mathrm{~Hz}, 1 \mathrm{H})$, $1.75(\mathrm{~d}, J=7.5 \mathrm{~Hz}, 4 \mathrm{H}), 1.52-1.60(\mathrm{~m}, 2 \mathrm{H}), 1.40-1.45$ (m, $1 \mathrm{H}), 1.30-1.35$ (m, $1 \mathrm{H})$, , $1.20-$ $1.26(\mathrm{~m}, 4 \mathrm{H}), 1.00-1.11(\mathrm{~m}, 3 \mathrm{H})$. 
(3S,4S)-1-Cyclohexyl-4-\{[5-(2,4-difluoro-phenyl)-isoxazole-3-carbonyl]-amino\}-

piperidine-3-carboxylic acid (1-pyrimidin-2-yl-cyclopropyl)-amide (21g) was synthesised in analogy to 7b by HATU amidation of the chiral acid 15 with 1-(pyrimidin-2-yl)cyclopropan1-amine. LC-MS method B: $t_{R}=0.94 \mathrm{~min} ;[\mathrm{M}+\mathrm{H}]+=551.21 . \mathrm{LC}-\mathrm{HRMS}: \mathrm{t}_{\mathrm{R}}=0.61 \mathrm{~min} ; \mathrm{m} / \mathrm{z}$ $=550.2551$, found $=551.2581 .[\mathrm{M}+\mathrm{H}] .{ }^{1} \mathrm{H}$ NMR $\left(400 \mathrm{MHz}, \mathrm{D}_{6}\right.$-DMSO) $\delta: 8.53(\mathrm{~s}, 1 \mathrm{H}), 8.50$ $(\mathrm{s}, 2 \mathrm{H}), 8.42(\mathrm{~s}, 1 \mathrm{H}), 8.08(\mathrm{~m}, 1 \mathrm{H}), 7.59(\mathrm{~m}, 1 \mathrm{H}), 7.34\left(\mathrm{td}, J_{1}=8.5 \mathrm{~Hz}, J_{2}=2.2 \mathrm{~Hz}, 1 \mathrm{H}\right)$, 7.16-7.19 (m, 2 H), 3.94-3.97 (m, $1 \mathrm{H}), 2.96$ (d, $J=10.5 \mathrm{~Hz}, 1 \mathrm{H}), 2.82$ (d, $J=10.5 \mathrm{~Hz}, 1 \mathrm{H})$, 2.60-2.68 (m, $1 \mathrm{H}), 2.26-2.40(\mathrm{~m}, 4 \mathrm{H}), 1.86(\mathrm{~d}, J=10.5 \mathrm{~Hz}, 1 \mathrm{H}), 1.72-1.76(\mathrm{~m}, 4 \mathrm{H}), 1.52-$ $1.60(\mathrm{~m}, 2 \mathrm{H}), 1.46-1.50(\mathrm{~m}, 1 \mathrm{H}), 1.36-1.40(\mathrm{~m}, 1 \mathrm{H}), 1.22(\mathrm{~m}, 3 \mathrm{H}), 1.07-1.11(\mathrm{~m}, 3 \mathrm{H})$.

\section{Preparation of compounds 28a-28e, 28g-31}

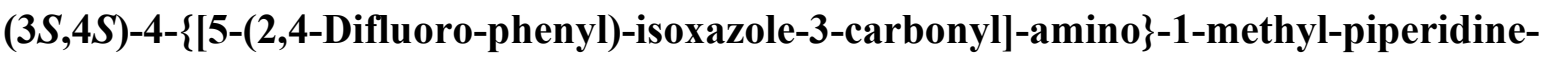
3-carboxylic acid (1-pyrimidin-2-yl-cyclopropyl)-amide (28a) was synthesised in analogy to $\mathbf{2 8 f}$ by reductive amination of $(3 S, 4 S)-4-\{[5-(2,4-$ difluoro-phenyl)-isoxazole-3-carbonyl $]$ amino -piperidine-3-carboxylic acid (1-pyrimidin-2-yl-cyclopropyl)-amide hydrochloride 27 with formaldehyde $36.5 \%$ aq. solution. LC-MS method $A: t_{R}=0.65 \min ;[M+H]^{+}=483.08$. LC-HRMS: $\mathrm{t}_{\mathrm{R}}=0.55 \mathrm{~min} ; \mathrm{m} / \mathrm{z}=482.1878$, found $=483.1951[\mathrm{M}+\mathrm{H}]^{+} .{ }^{1} \mathrm{H} \mathrm{NMR}(400 \mathrm{MHz}$, D6-DMSO) $\delta: 8.54(\mathrm{~d}, J=8.5 \mathrm{~Hz}, 1 \mathrm{H}), 8.50(\mathrm{~d}, J=4.9 \mathrm{~Hz}, 2 \mathrm{H}), 8.44(\mathrm{~m}, 1 \mathrm{H}), 8.08(\mathrm{~m}, 1 \mathrm{H})$, 7.56-7.62 (m, $1 \mathrm{H}), 7.34\left(\mathrm{td}, J_{1}=8.5 \mathrm{~Hz}, J_{2}=2.2 \mathrm{~Hz}, 1 \mathrm{H}\right), 7.18(\mathrm{~d}, J=4.8, \mathrm{~Hz}, 1 \mathrm{H}), 7.16(\mathrm{~d}$, $J=3.0 \mathrm{~Hz}, 1 \mathrm{H}), 3.92-4.01(\mathrm{~m}, 1 \mathrm{H}), 2.91(\mathrm{~d}, J=9.0 \mathrm{~Hz} .1 \mathrm{H}), 2.76-2.80(\mathrm{~m}, 1 \mathrm{H}), 2.73(\mathrm{~m}, 1$ H), 2.18-2.24 (m, $3 \mathrm{H}), 2.07(\mathrm{t}, J=11.5 \mathrm{~Hz}, 1 \mathrm{H}), 1.96(\mathrm{t}, J=11.3 \mathrm{~Hz}, 1 \mathrm{H}), 1.83\left(\mathrm{dd}, J_{1}=12.8\right.$ $\left.\mathrm{Hz}, J_{2}=4.3 \mathrm{~Hz}, 1 \mathrm{H}\right), 1.63(\mathrm{~m}, J=3.8 \mathrm{~Hz}, 1 \mathrm{H}), 1.46-1.50(\mathrm{~m}, 1 \mathrm{H}), 1.35-1.40(\mathrm{~m}, 1 \mathrm{H}), 1.04-$ $1.12(\mathrm{~m}, 2 \mathrm{H})$.

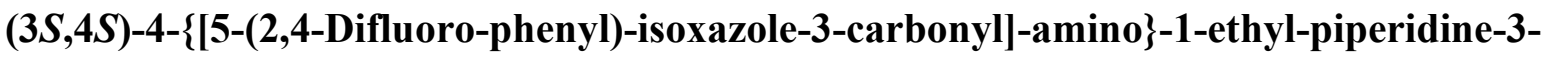
carboxylic acid (1-pyrimidin-2-yl-cyclopropyl)-amide (28b) was synthesised by reductive 
amination of the amine hydrochloride 27 with acetaldehyde. LC-MS method A: $t_{R}=0.65$ min; $[\mathrm{M}+\mathrm{H}]^{+}=497.03 . \mathrm{LC}-\mathrm{HRMS}: \mathrm{t}_{\mathrm{R}}=0.56 \mathrm{~min} ; \mathrm{m} / \mathrm{z}=496.2034$, found $=497.2105[\mathrm{M}+\mathrm{H}]^{+} .{ }^{1} \mathrm{H}$ NMR (500 MHz, D6-DMSO) $\delta: 8.57$ (d, $J=8.4 \mathrm{~Hz}, 1 \mathrm{H}), 8.50(\mathrm{~d}, J=4.8 \mathrm{~Hz}, 2 \mathrm{H}), 8.46$ (s, 1 H), $8.08(\mathrm{~m}, 1 \mathrm{H}), 7.60(\mathrm{~m}, 1 \mathrm{H}), 7.34\left(\mathrm{td}, J_{1}=2.0 \mathrm{~Hz}, J_{2}=8.4 \mathrm{~Hz}, 1 \mathrm{H}\right), 7.18(\mathrm{~d}, J=4.8 \mathrm{~Hz}, 1$ H), $7.17(\mathrm{~d}, J=3.0 \mathrm{~Hz}, 1 \mathrm{H}), 3.97-4.03(\mathrm{~m}, 1 \mathrm{H}), 3.03(\mathrm{~d}, J=11.0 \mathrm{~Hz}, 1 \mathrm{H}), 2.90-2.94(\mathrm{~m}, 1$ H), 2.69-2.75 (m, $1 \mathrm{H}), 2.37-2.40(\mathrm{~m}, 2 \mathrm{H}), 2.06-2.12(\mathrm{~m}, 1 \mathrm{H}), 1.95-2.01(\mathrm{~m}, 1 \mathrm{H}), 1.86(\mathrm{~d}, J$ $=11.0 \mathrm{~Hz}, 1 \mathrm{H}), 1.56-1.64(\mathrm{~m}, 1 \mathrm{H}), 1.37-1.50(\mathrm{~m}, 2 \mathrm{H}), 1.22-1.29(\mathrm{~m}, 1 \mathrm{H}), 1.06-1.10(\mathrm{~m}, 1$ $\mathrm{H}), 1.03(\mathrm{t}, J=7.1 \mathrm{~Hz}, 3 \mathrm{H})$.

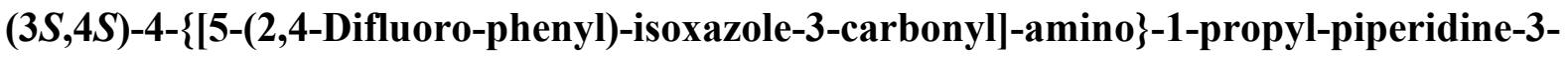
carboxylic acid (1-pyrimidin-2-yl-cyclopropyl)-amide (28c). To a solution of $(3 S, 4 S)-4-\{[5-$ (2,4-difluoro-phenyl)-isoxazole-3-carbonyl]-amino -piperidine-3-carboxylic acid (1pyrimidin-2-yl-cyclopropyl)-amide hydrochloride 27 (70 mg, $0.14 \mathrm{mmol}$ in EtOH (4 mL) was added 1-iodopropane $(0.0137 \mathrm{~mL}, 0.14 \mathrm{mmol})$ followed by DIPEA $(0.0712 \mathrm{~mL}, 0.416 \mathrm{mmol})$. The mixture was stirred overnight at $65^{\circ} \mathrm{C}$. The mixture was concentrated, the residues dissolved in DMF and purified by prep. HPLC under basic conditions to yield 28c (35 mg, $50 \%$ ) as a white solid. LC-MS method $\mathrm{A}: \mathrm{t}_{\mathrm{R}}=0.69 \mathrm{~min} ;[\mathrm{M}+\mathrm{H}]^{+}=511.28$. LC-HRMS: $\mathrm{t}_{\mathrm{R}}=$ $0.57 \mathrm{~min} ; \mathrm{m} / \mathrm{z}=510.2191$, found $=511.2266[\mathrm{M}+\mathrm{H}]^{+} .{ }^{1} \mathrm{H}$ NMR $\left(400 \mathrm{MHz}, \mathrm{D}_{6}-\mathrm{DMSO}\right) \delta$ : $8.55(\mathrm{~d}, J=8.5 \mathrm{~Hz}, 1 \mathrm{H}), 8.50(\mathrm{~d}, J=4.9 \mathrm{~Hz}, 2 \mathrm{H}), 8.45(\mathrm{~s}, 1 \mathrm{H}), 8.08\left(\mathrm{td}, J_{1}=8.7 \mathrm{~Hz}, J_{2}=\right.$ 6.4Hz, $1 \mathrm{H}), 7.60(\mathrm{~m}, 1 \mathrm{H}), 7.34\left(\mathrm{td}, J_{1}=8.2 \mathrm{~Hz}, J_{2}=2.1 \mathrm{~Hz}, 1 \mathrm{H}\right), 7.18(\mathrm{~m}, 1 \mathrm{H}), 7.16(\mathrm{~d}, J=$ 2.7 Hz, $1 \mathrm{H}), 3.99$ (m, $1 \mathrm{H}), 2.99(\mathrm{~d}, J=9.8 \mathrm{~Hz}, 1 \mathrm{H}), 2.87$ (d, $J=11.3 \mathrm{~Hz}, 1 \mathrm{H}), 2.67-2.73(\mathrm{~m}$, $1 \mathrm{H}), 2.28(\mathrm{~m}, 2 \mathrm{H}), 2.08(\mathrm{t}, J=11.6 \mathrm{~Hz}, 1 \mathrm{H}), 1.96(\mathrm{~m}, J=12.0 \mathrm{~Hz}, 1 \mathrm{H}), 1.84\left(\mathrm{dd}, J_{1}=12.8\right.$ $\left.\mathrm{Hz}, J_{2}=4.0 \mathrm{~Hz}, 1 \mathrm{H}\right), 1.60(\mathrm{~m}, 1 \mathrm{H}), 1.43-1.50(\mathrm{~m}, 3 \mathrm{H}), 1.36-1.41(\mathrm{~m}, 1 \mathrm{H}), 1.08(\mathrm{~m}, 2 \mathrm{H})$, $0.88(\mathrm{t}, J=7.3 \mathrm{~Hz}, 3 \mathrm{H})$.

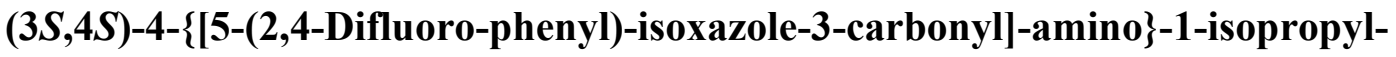
piperidine-3-carboxylic acid (1-pyrimidin-2-yl-cyclopropyl)-amide (28d) was synthesised 
by reductive amination of the amine hydrochloride 27 with acetone. LC-MS method A: $t_{R}=$ $0.68 \mathrm{~min} ;[\mathrm{M}+\mathrm{H}]^{+}=511.27 . \mathrm{LC}-\mathrm{HRMS}: \mathrm{t}_{\mathrm{R}}=0.56 \mathrm{~min} ; \mathrm{m} / \mathrm{z}=510.2191$, found $=511.2275[\mathrm{M}$ $+\mathrm{H}]^{+} .{ }^{1} \mathrm{H}$ NMR (400 MHz, D6-DMSO) $\delta: 8.54(\mathrm{~d}, J=8.5 \mathrm{~Hz}, 1 \mathrm{H}), 8.50(\mathrm{~d}, J=4.9 \mathrm{~Hz}, 2 \mathrm{H})$, $8.44(\mathrm{~s}, 1 \mathrm{H}), 8.08\left(\mathrm{td}, J_{1}=8.7 \mathrm{~Hz}, J_{2}=6.4 \mathrm{~Hz}, 1 \mathrm{H}\right), 7.60(\mathrm{~m}, 1 \mathrm{H}), 7.34\left(\mathrm{td}, J_{1}=8.0 \mathrm{~Hz}, J_{2}=\right.$ $2.0 \mathrm{~Hz}, 1 \mathrm{H}), 7.18(\mathrm{~m}, 1 \mathrm{H}), 7.16(\mathrm{~m}, 1 \mathrm{H}), 3.96(\mathrm{~m}, 1 \mathrm{H}), 2.92(\mathrm{~d}, J=9.7 \mathrm{~Hz}, 1 \mathrm{H}), 2.73-2.80$ (m, 2 H), 2.62-2.68 (m, $1 \mathrm{H}), 2.29(\mathrm{t}, J=11.2 \mathrm{~Hz}, 1 \mathrm{H}), 2.19(\mathrm{~m}, 1 \mathrm{H}), 1.85-1.89(\mathrm{~m}, 1 \mathrm{H}), 1.55$ $\left(\mathrm{dd}, J_{1}=11.9 \mathrm{~Hz}, J_{2}=3.7 \mathrm{~Hz}, 1 \mathrm{H}\right), 1.46-1.50(\mathrm{~m}, 1 \mathrm{H}), 1.36-1.40(\mathrm{~m}, 1 \mathrm{H}), 1.05-1.13(\mathrm{~m}, 2$ $\mathrm{H}), 0.99(\mathrm{~d}, J=6.5 \mathrm{~Hz}, 6 \mathrm{H})$

(3S,4S)-1-Cyclopropyl-4-\{[5-(2,4-difluoro-phenyl)-isoxazole-3-carbonyl]-amino\}piperidine-3-carboxylic acid (1-pyrimidin-2-yl-cyclopropyl)-amide hydrochloride (28e) was synthesised by reductive amination of the amine hydrochloride 27 with (1ethoxycyclopropoxy)trimethylsilane. LC-MS method B: $\mathrm{t}_{\mathrm{R}}=0.88 \mathrm{~min} ;[\mathrm{M}+\mathrm{H}]^{+}=509.15$. LCHRMS: $\mathrm{t}_{\mathrm{R}}=0.63 \mathrm{~min} ; \mathrm{m} / \mathrm{z}=508.2034$, found $=509.2120[\mathrm{M}+\mathrm{H}]^{+} .{ }^{1} \mathrm{H}$ NMR $\left(400 \mathrm{MHz}, \mathrm{D}_{6^{-}}\right.$ DMSO) $\delta: 8.88(\mathrm{~m}, 1 \mathrm{H}), 8.79(\mathrm{~s}, 1 \mathrm{H}), 8.53(\mathrm{~d}, J=4.8 \mathrm{~Hz}, 2 \mathrm{H}), 8.06(\mathrm{~m}, 1 \mathrm{H}), 7.57(\mathrm{~m}, 1 \mathrm{H})$, 7.31-7.35 (m, $2 \mathrm{H}), 7.21$ (t, $J=4.8 \mathrm{~Hz}, 1 \mathrm{H}), 4.22-4.31(\mathrm{~m}, 1 \mathrm{H}), 3.26-3.51$ (m, $3 \mathrm{H}), 2.80-2.87$ (m, $1 \mathrm{H}), 1.99-2.10(\mathrm{~m}, 3 \mathrm{H}), 1.49-1.51(\mathrm{~m}, 1 \mathrm{H}), 1.36-1.39$ (m, $1 \mathrm{H}), 1.06-1.16(\mathrm{~m}, 5 \mathrm{H}), 0.81$ $(\mathrm{d}, J=6.4 \mathrm{~Hz}, 3 \mathrm{H})$.

\section{(3S,4S)-1-Cyclobutyl-4-\{[5-(2,4-difluoro-phenyl)-isoxazole-3-carbonyl]-amino\}-}

piperidine-3-carboxylic acid (1-pyrimidin-2-yl-cyclopropyl)-amide (28g) was synthesised by reductive amination of the amine hydrochloride 27 with cyclobutanone. LC-MS method A: $t_{R}=0.68 \mathrm{~min} ;[\mathrm{M}+\mathrm{H}]^{+}=523.04$. LC-HRMS: $\mathrm{t}_{\mathrm{R}}=0.57 \mathrm{~min} ; \mathrm{m} / \mathrm{z}=522.2191$, found $=523.2266$ $[\mathrm{M}+\mathrm{H}]^{+} .{ }^{1} \mathrm{H}$ NMR $\left(400 \mathrm{MHz}, \mathrm{D}_{6}\right.$-DMSO $) \delta: 8.57(\mathrm{~d}, J=8.5 \mathrm{~Hz}, 1 \mathrm{H}), 8.50(\mathrm{~d}, J=4.8 \mathrm{~Hz}, 2$ H), $8.45(\mathrm{~s}, 1 \mathrm{H}), 8.08(\mathrm{~m}, 1 \mathrm{H}), 7.60(\mathrm{~m}, 1 \mathrm{H}), 7.34\left(\mathrm{td}, J_{1}=8.4 \mathrm{~Hz}, J_{2}=2.1 \mathrm{~Hz}, 1 \mathrm{H}\right), 7.18$ $(\mathrm{m}, 1 \mathrm{H}), 7.16(\mathrm{~d}, J=2.6 \mathrm{~Hz}, 1 \mathrm{H}), 3.98(\mathrm{~m}, 1 \mathrm{H}), 2.92(\mathrm{~d}, J=9.1 \mathrm{~Hz}, 1 \mathrm{H}), 2.80(\mathrm{~d}, J=10.8$ Hz, $1 \mathrm{H}), 2.63-2.74(\mathrm{~m}, 2 \mathrm{H}), 1.99(\mathrm{~d}, J=3.9 \mathrm{~Hz}, 2 \mathrm{H}), 1.79-1.92(\mathrm{~m}, 5 \mathrm{H}), 1.60-1.65(\mathrm{~m}, 2 \mathrm{H})$, 
$1.55\left(\mathrm{dd}, J_{1}=12.1 \mathrm{~Hz}, J_{2}=3.7 \mathrm{~Hz}, 1 \mathrm{H}\right), 1.46-1.50(\mathrm{~m}, 1 \mathrm{H}), 1.35-1.39(\mathrm{~m}, 1 \mathrm{H}), 1.04-1.12(\mathrm{~m}$, $2 \mathrm{H})$.

(3S,4S)-1-Cyclobutylmethyl-4-\{[5-(2,4-difluoro-phenyl)-isoxazole-3-carbonyl]-amino\}piperidine-3-carboxylic acid (1-pyrimidin-2-yl-cyclopropyl)-amide hydrochloride (28h) was synthesised by reductive amination of the amine hydrochloride 27 with cyclobutanecarbaldehyde. LC-MS method $\mathrm{B}: \mathrm{t}_{\mathrm{R}}=0.97 \mathrm{~min} ;[\mathrm{M}+\mathrm{H}]^{+}=537.17 . \mathrm{LC}-\mathrm{HRMS}: \mathrm{t}_{\mathrm{R}}$ $=0.60 \mathrm{~min} ; \mathrm{m} / \mathrm{z}=536.2347$, found $=537.2430[\mathrm{M}+\mathrm{H}]^{+} .{ }^{1} \mathrm{H}$ NMR $\left(400 \mathrm{MHz}, \mathrm{D}_{6}-\mathrm{DMSO}\right) \delta$ : 10.62-10.67 (m, $1 \mathrm{H}), 8.91(\mathrm{~d}, J=8.6 \mathrm{~Hz}, 1 \mathrm{H}), 8.83(\mathrm{~s}, 1 \mathrm{H}), 8.52(\mathrm{~d}, J=4.9 \mathrm{~Hz}, 2 \mathrm{H}), 8.06$ (m, $1 \mathrm{H}), 7.55-7.60(\mathrm{~m}, 1 \mathrm{H}), 7.31-7.35(\mathrm{~m}, 2 \mathrm{H}), 7.20(\mathrm{t}, J=4.9 \mathrm{~Hz}, 1 \mathrm{H}), 4.22-4.26(\mathrm{~m}, 1 \mathrm{H})$, 3.10-3.29 (m, $5 \mathrm{H}), 2.80-2.84(\mathrm{~m}, 1 \mathrm{H}), 1.98-2.13(\mathrm{~m}, 5 \mathrm{H}), 1.76-1.93(\mathrm{~m}, 5 \mathrm{H}), 1.50\left(\mathrm{dd}, J_{1}=\right.$ $\left.9.8 \mathrm{~Hz}, J_{2}=4.1 \mathrm{~Hz}, 1 \mathrm{H}\right), 1.34-1.37(\mathrm{~m}, 1 \mathrm{H}), 1.08-1.11(\mathrm{~m}, 2 \mathrm{H})$.

(3S,4S)-1-Cyclopentyl-4-\{[5-(2,4-difluoro-phenyl)-isoxazole-3-carbonyl]-amino\}piperidine-3-carboxylic acid (1-pyrimidin-2-yl-cyclopropyl)-amide hydrochloride (28i) was synthesised by reductive amination of the amine hydrochloride 27 with cyclopentanone. LC-MS method A: $t_{R}=0.75 \mathrm{~min} ;[\mathrm{M}+\mathrm{H}]^{+}=537.19$. LC-HRMS: $\mathrm{t}_{\mathrm{R}}=0.59 \mathrm{~min} ; \mathrm{m} / \mathrm{z}=536.2347$, found $=537.2423[\mathrm{M}+\mathrm{H}]^{+} .{ }^{1} \mathrm{H}$ NMR $\left(400 \mathrm{MHz}, \mathrm{D}_{6}-\mathrm{DMSO}\right) \delta$ : 10.66-10.77 (s, $\left.1 \mathrm{H}\right), 8.91(\mathrm{~d}$, $J=8.4 \mathrm{~Hz}, 1 \mathrm{H}), 8.79(\mathrm{~s}, 1 \mathrm{H}), 8.51(\mathrm{~d}, J=4.9 \mathrm{~Hz}, 2 \mathrm{H}), 8.07\left(\mathrm{td}, J_{1}=8.7 \mathrm{~Hz}, J_{2}=6.4 \mathrm{~Hz}, 1\right.$ H), $7.59(\mathrm{~m}, 1 \mathrm{H}), 7.35\left(\mathrm{dd}, J_{1}=8.4 \mathrm{~Hz}, J_{2}=2.2 \mathrm{~Hz}, 1 \mathrm{H}\right), 7.31(\mathrm{~d}, J=2.8 \mathrm{~Hz}, 1 \mathrm{H}), 7.20(\mathrm{t}, J$ $=4.9 \mathrm{~Hz}, 1 \mathrm{H}), 4.24-4.33(\mathrm{~m}, 1 \mathrm{H}), 3.50-3.54(\mathrm{~m}, 3 \mathrm{H}), 3.15-3.30(\mathrm{~m}, 3 \mathrm{H}), 1.99-2.10(\mathrm{~m}, 4 \mathrm{H})$, 1.83 (m, 2 H), 1.68-1.77 (m, 2 H), 1.49-1.60 (m, 3 H), 1.36-1.40 (m, $1 \mathrm{H}), 1.08-1.11(\mathrm{~m}, 2 \mathrm{H})$.

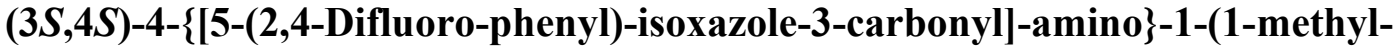
cyclopropylmethyl)-piperidine-3-carboxylic acid (1-pyrimidin-2-yl-cyclopropyl)-amide (28j) was synthesised by reductive amination of the amine hydrochloride 27 with 1methylcyclopropane-1-carbaldehyde. $L C-M S$ method A: $t_{R}=0.71 \mathrm{~min} ;[\mathrm{M}+\mathrm{H}]^{+}=537.04$. LCHRMS: $\mathrm{t}_{\mathrm{R}}=0.59 \mathrm{~min} ; \mathrm{m} / \mathrm{z}=536.2347$, found $=537.2416[\mathrm{M}+\mathrm{H}]^{+} .{ }^{1} \mathrm{H}$ NMR $\left(400 \mathrm{MHz}, \mathrm{D}_{6}-\right.$ 
DMSO) $\delta: 8.56(\mathrm{~d}, J=8.5 \mathrm{~Hz}, 1 \mathrm{H}), 8.51(\mathrm{~d}, J=4.8 \mathrm{~Hz}, 2 \mathrm{H}), 8.48(\mathrm{~s}, 1 \mathrm{H}), 8.08\left(\mathrm{td}, J_{1}=8.7\right.$ $\left.\mathrm{Hz}, J_{2}=6.5 \mathrm{~Hz}, 1 \mathrm{H}\right), 7.60(\mathrm{~m}, 1 \mathrm{H}), 7.34\left(\mathrm{td}, J_{1}=8.4 \mathrm{~Hz}, J_{2}=2.3 \mathrm{~Hz}, 1 \mathrm{H}\right), 7.19(\mathrm{~d}, J=4.8 \mathrm{~Hz}$, $1 \mathrm{H}), 7.17(\mathrm{~d}, J=3.0 \mathrm{~Hz}, 1 \mathrm{H}), 3.99(\mathrm{~m}, 1 \mathrm{H}), 3.12\left(\mathrm{dd}, J_{1}=11.1 \mathrm{~Hz}, J_{2}=2.0 \mathrm{~Hz}, 1 \mathrm{H}\right), 3.00$ $(\mathrm{d}, J=11.0 \mathrm{~Hz}, 1 \mathrm{H}), 2.73\left(\mathrm{td}, J_{1}=10.9 \mathrm{~Hz}, J_{2}=3.6 \mathrm{~Hz}, 1 \mathrm{H}\right), 2.23(\mathrm{~m}, 1 \mathrm{H}), 2.09(\mathrm{~d}, J=11.9$ Hz, $1 \mathrm{H}), 2.04$ (m, $1 \mathrm{H}), 1.84-1.94$ (m, 2 H), 1.57-1.67 (m, $1 \mathrm{H}), 1.48-1.52$ (m, $1 \mathrm{H}), 1.35-1.39$ (m, $1 \mathrm{H}), 1.07-1.11(\mathrm{~m}, 5 \mathrm{H}), 0.26-0.32(\mathrm{~m}, 4 \mathrm{H})$.

\section{$(3 S, 4 S)-4-\{[5-(2,4-D i f l u o r o-p h e n y l)-i s o x a z o l e-3-c a r b o n y l]-a m i n o\}-1-(1-f l u o r o-$}

cyclopropylmethyl)-piperidine-3-carboxylic acid (1-pyrimidin-2-yl-cyclopropyl)-amide (28k) was synthesised by reductive amination of the amine hydrochloride 27 with 1fluorocyclopropane-1-carbaldehyde._LC-MS method A: $\mathrm{t}_{\mathrm{R}}=0.71 \mathrm{~min} ;[\mathrm{M}+\mathrm{H}]^{+}=541.24$. LCHRMS: $\mathrm{t}_{\mathrm{R}}=0.58 \mathrm{~min} ; \mathrm{m} / \mathrm{z}=540.2097$, found $=541.2179[\mathrm{M}+\mathrm{H}]^{+} .{ }^{1} \mathrm{H}$ NMR $\left(400 \mathrm{MHz}, \mathrm{D}_{6}-\right.$ DMSO) $\delta: 8.57(\mathrm{~d}, J=8.5 \mathrm{~Hz}, 1 \mathrm{H}), 8.51(\mathrm{~d}, J=4.8 \mathrm{~Hz}, 2 \mathrm{H}), 8.48(\mathrm{~s}, 1 \mathrm{H}), 8.08(\mathrm{~m}, 1 \mathrm{H}), 7.60$ $(\mathrm{m}, 1 \mathrm{H}), 7.34\left(\mathrm{td}, J_{1}=8.6 \mathrm{~Hz}, J_{2}=2.2 \mathrm{~Hz}, 1 \mathrm{H}\right), 7.18(\mathrm{t}, J=4.8 \mathrm{~Hz}, 1 \mathrm{H}), 7.16(\mathrm{~d}, J=2.9 \mathrm{~Hz}$, $1 \mathrm{H}), 4.00(\mathrm{~m}, 1 \mathrm{H}), 3.16(\mathrm{~d}, J=9.3 \mathrm{~Hz}, 1 \mathrm{H}), 3.01(\mathrm{~d}, J=11.3 \mathrm{~Hz}, 1 \mathrm{H}), 2.71-2.78(\mathrm{~m}, 3 \mathrm{H})$, $2.31(\mathrm{t}, J=11.5 \mathrm{~Hz}, 1 \mathrm{H}), 2.20(\mathrm{t}, J=11.8 \mathrm{~Hz}, 1 \mathrm{H}), 1.86(\mathrm{~d}, J=9.5 \mathrm{~Hz}, 1 \mathrm{H}), 1.63(\mathrm{~m}, 1 \mathrm{H})$, 1.48-1.52 (m, $1 \mathrm{H}), 1.35-1.39(\mathrm{~m}, 1 \mathrm{H}), 1.02-1.10(\mathrm{~m}, 3 \mathrm{H}), 0.99(\mathrm{~m}, J=6.9 \mathrm{~Hz}, 1 \mathrm{H}), 0.68(\mathrm{~m}$, $2 \mathrm{H})$.

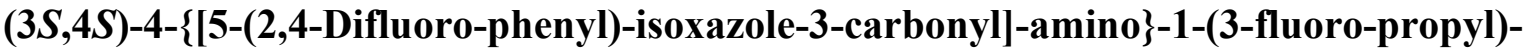
piperidine-3-carboxylic acid (1-pyrimidin-2-yl-cyclopropyl)-amide (28I) was synthesised by N-alkylation of the amine hydrochloride 27 with 1-bromo-3-fluoro-propane. LC-MS method $\mathrm{A}: \mathrm{t}_{\mathrm{R}}=0.67 \mathrm{~min} ;[\mathrm{M}+\mathrm{H}]^{+}=529.06 . \mathrm{LC}-\mathrm{HRMS}: \mathrm{t}_{\mathrm{R}}=0.57 \mathrm{~min} ; \mathrm{m} / \mathrm{z}=528.2097$, found $=529.2179[\mathrm{M}+\mathrm{H}]^{+} .1 \mathrm{H}$ NMR $\left(400 \mathrm{MHz}, \mathrm{D}_{6}-\mathrm{DMSO}\right) \delta: 11.01-11.12(\mathrm{~s}, 1 \mathrm{H}), 8.91-8.93(\mathrm{~m}$, $1 \mathrm{H}), 8.79-8.82(\mathrm{~m}, 1 \mathrm{H}), 8.51(\mathrm{~d}, J=4.8 \mathrm{~Hz}, 2 \mathrm{H}), 8.07(\mathrm{~m}, 1 \mathrm{H}), 7.59(\mathrm{t}, J=9.5 \mathrm{~Hz}, 1 \mathrm{H})$, 7.32-7.36 (m, $2 \mathrm{H}), 7.20(\mathrm{t}, J=4.8 \mathrm{~Hz}, 1 \mathrm{H}), 4.61(\mathrm{t}, J=5.5 \mathrm{~Hz}, 1 \mathrm{H}), 4.49(\mathrm{t}, J=5.3 \mathrm{~Hz}, 1 \mathrm{H})$, 
4.22-4.32 (m, $1 \mathrm{H}), 3.54-3.60(\mathrm{~m}, 2 \mathrm{H}), 3.19-3.26(\mathrm{~m}, 4 \mathrm{H}), 1.99-2.26(\mathrm{~m}, 5 \mathrm{H}), 1.45-1.51$ (m, $1 \mathrm{H}), 1.35-1.41(\mathrm{~m}, 1 \mathrm{H}), 1.05-1.13(\mathrm{~m}, 2 \mathrm{H})$.

(3S,4S)-1-(3,3-Difluoro-cyclobutyl)-4-\{[5-(2,4-difluoro-phenyl)-isoxazole-3-carbonyl]amino\}-piperidine-3-carboxylic acid (1-pyrimidin-2-yl-cyclopropyl)-amide (28m) was synthesised by reductive amination of the amine hydrochloride 27 with 3,3difluorocyclobutan-1-one._LC-MS method A: $t_{R}=0.69 \mathrm{~min} ;[\mathrm{M}+\mathrm{H}]^{+}=558.96$. LC-HRMS: $\mathrm{t}_{\mathrm{R}}$ $=0.63 \mathrm{~min} ; \mathrm{m} / \mathrm{z}=558.2003$, found $=559.2068[\mathrm{M}+\mathrm{H}]^{+} .{ }^{1} \mathrm{H}$ NMR $\left(400 \mathrm{MHz}, \mathrm{D}_{6}-\mathrm{DMSO}\right) \delta$ : $8.60(\mathrm{~d}, J=8.3 \mathrm{~Hz}, 1 \mathrm{H}), 8.50(\mathrm{~d}, J=4.9 \mathrm{~Hz}, 2 \mathrm{H}), 8.46(\mathrm{~s}, 1 \mathrm{H}), 8.08\left(\mathrm{td}, J_{1}=8.7 \mathrm{~Hz}, J_{2}=6.5\right.$ $\mathrm{Hz}, 1 \mathrm{H}), 7.60(\mathrm{~m}, 1 \mathrm{H}), 7.34\left(\mathrm{td}, J_{1}=8.4 \mathrm{~Hz}, J_{2}=2.2 \mathrm{~Hz}, 1 \mathrm{H}\right), 7.18(\mathrm{~d}, J=4.9 \mathrm{~Hz}, 1 \mathrm{H}), 7.16$ (m, $1 \mathrm{H}), 4.01(\mathrm{~m}, 1 \mathrm{H}), 2.95(\mathrm{~d}, J=9.3 \mathrm{~Hz}, 1 \mathrm{H}), 2.84(\mathrm{~d}, J=11.0 \mathrm{~Hz}, 1 \mathrm{H}), 2.67-2.79$ (m, 4 H), 2.33-2.47 (m, $2 \mathrm{H}), 2.04(\mathrm{t}, J=11.5 \mathrm{~Hz}, 1 \mathrm{H}), 1.85-1.94(\mathrm{~m}, 2 \mathrm{H}), 1.53-1.63(\mathrm{~m}, 1 \mathrm{H}), 1.46-$ 1.50 (m, $1 \mathrm{H}), 1.36-1.40$ (m, $1 \mathrm{H}), 1.04-1.14$ (m, $2 \mathrm{H})$.

$(3 S, 4 S)-4-\{[5-(2,4-D i f l u o r 0-p h e n y l)-i s o x a z o l e-3-c a r b o n y l]-a m i n o\}-1-(2-m e t h o x y-e t h y l)-$ piperidine-3-carboxylic acid (1-pyrimidin-2-yl-cyclopropyl)-amide (28n) was synthesised by $\mathrm{N}$-alkylation of the amine hydrochloride 27 with 1-bromo-2-methoxyethane. LC-MS method $A: t_{R}=0.63 \mathrm{~min} ;[\mathrm{M}+\mathrm{H}]^{+}=527.20 . \mathrm{LC}-\mathrm{HRMS}: \mathrm{t}_{\mathrm{R}}=0.56 \mathrm{~min} ; \mathrm{m} / \mathrm{z}=526.2140$, found $=527.2198[\mathrm{M}+\mathrm{H}]^{+} .1 \mathrm{H}$ NMR $\left(400 \mathrm{MHz}, \mathrm{D}_{6}-\mathrm{DMSO}\right) \delta: 8.55(\mathrm{~d}, J=8.4 \mathrm{~Hz}, 1 \mathrm{H}), 8.50(\mathrm{~d}, J$ $=4.8 \mathrm{~Hz}, 2 \mathrm{H}), 8.45(\mathrm{~s}, 1 \mathrm{H}), 8.08(\mathrm{~m}, 1 \mathrm{H}), 7.59(\mathrm{~m}, 1 \mathrm{H}), 7.34\left(\mathrm{td}, J_{1}=8.5 \mathrm{~Hz}, J_{2}=1.8 \mathrm{~Hz}, 1\right.$ H), $7.18(\mathrm{~m}, 1 \mathrm{H}), 7.16(\mathrm{~d}, J=3.1 \mathrm{~Hz}, 1 \mathrm{H}), 3.98(\mathrm{~m}, 1 \mathrm{H}), 3.44(\mathrm{t}, J=5.7 \mathrm{~Hz}, 2 \mathrm{H}), 3.25(\mathrm{~s}, 3$ H), $3.01(\mathrm{~d}, J=9.5 \mathrm{~Hz}, 1 \mathrm{H}), 2.90(\mathrm{~d}, J=11.1 \mathrm{~Hz}, 1 \mathrm{H}), 2.70\left(\mathrm{td}, J_{1}=3.6 \mathrm{~Hz}, J_{2}=11.0 \mathrm{~Hz}, 1\right.$ H), $2.57(\mathrm{~m}, 2 \mathrm{H}), 2.20(\mathrm{t}, J=11.3 \mathrm{~Hz}, 1 \mathrm{H}), 2.08(\mathrm{t}, J=11.0 \mathrm{~Hz}, 1 \mathrm{H}), 1.83\left(\mathrm{dd}, J_{1}=2.8 \mathrm{~Hz}\right.$, $\left.J_{2}=12.3 \mathrm{~Hz}, 1 \mathrm{H}\right), 1.54-1.65(\mathrm{~m}, 1 \mathrm{H}), 1.47-1.51(\mathrm{~m}, 1 \mathrm{H}), 1.36-1.40(\mathrm{~m}, 1 \mathrm{H}), 1.04-1.13(\mathrm{~m}$, $2 \mathrm{H})$.

(3S,4S)-1-Acetyl-4-\{[5-(2,4-difluoro-phenyl)-isoxazole-3-carbonyl]-amino\}-piperidine-3carboxylic acid (1-pyrimidin-2-yl-cyclopropyl)-amide (280) was synthesised by N-acylation 
of the amine hydrochloride 27 with acetic anhydride in DCM in the presence of triethylamine. LC-MS method A: $\mathrm{t}_{\mathrm{R}}=0.75 \mathrm{~min} ;[\mathrm{M}+\mathrm{H}]^{+}=511.04 . \mathrm{LC}-\mathrm{HRMS}: \mathrm{t}_{\mathrm{R}}=0.79 \mathrm{~min} ; \mathrm{m} / \mathrm{z}=510.1827$, found $=511.1913[\mathrm{M}+\mathrm{H}]^{+} .{ }^{1} \mathrm{H}$ NMR $\left(400 \mathrm{MHz}, \mathrm{D}_{6}\right.$-DMSO) $\delta: 8.61(\mathrm{~m}, 1 \mathrm{H}), 8.56(\mathrm{~s}, 1 \mathrm{H})$, $8.53(\mathrm{~d}, J=4.7 \mathrm{~Hz}, 2 \mathrm{H}), 8.05-8.11(\mathrm{~m}, 1 \mathrm{H}), 7.60(\mathrm{t}, J=10.4 \mathrm{~Hz}, 1 \mathrm{H}), 7.34(\mathrm{t}, J=8.5 \mathrm{~Hz}, 1$ H), 7.15-7.21 (m, $2 \mathrm{H}), 4.57(\mathrm{~d}, J=11.3 \mathrm{~Hz}, 1 \mathrm{H}), 4.21-4.29(\mathrm{~m}, 1 \mathrm{H}), 3.87(\mathrm{~d}, J=13.0 \mathrm{~Hz}, 1$ H), $3.18(\mathrm{~m}, 1 \mathrm{H}), 2.62-2.72(\mathrm{~m}, 2 \mathrm{H}), 2.09(\mathrm{~s}, 3 \mathrm{H}), 1.89(\mathrm{~m}, J=13.5 \mathrm{~Hz}, 1 \mathrm{H}), 1.50-1.56(\mathrm{~m}$, $2 \mathrm{H}), 1.35-1.43(\mathrm{~m}, 1 \mathrm{H}), 1.04-1.17(\mathrm{~m}, 2 \mathrm{H})$.

(3R,4R)-1-Cyclopropylmethyl-4-\{[5-(2,4-difluoro-phenyl)-isoxazole-3-carbonyl]-amino\}piperidine-3-carboxylic acid (1-pyrimidin-2-yl-cyclopropyl)-amide (29) was synthesised by reductive amination of $(3 R, 4 R)-4-\{[5-(2,4-D i f l u o r o-p h e n y l)-i s o x a z o l e-3-c a r b o n y l]-$ amino\}-piperidine-3-carboxylic acid (1-pyrimidin-2-yl-cyclopropyl)-amide hydrochloride with cyclopropanecarboxaldehyde. LC-MS method A: $t_{R}=0.69 \min ;[M+H]^{+}=523.03$. Chiral HPLC: $\mathrm{t}_{\mathrm{R}}=4.6 \mathrm{~min} ; 99 \%$ ee; column: ChiralPak IC 4.6x250 mm, $5 \mu \mathrm{M}$; detector wavelength: $254 \mathrm{~nm}$; eluent: 10\% heptane 0.05\% DEA; 90\% ethanol 0.05\% DEA; flow: $1.2 \mathrm{~mL} / \mathrm{min}$; BPR: 150 bar; temperature: $25^{\circ} \mathrm{C}$; injection volume: $6 \mu \mathrm{l}$. LC-HRMS: $\mathrm{t}_{\mathrm{R}}=0.57 \mathrm{~min} ; \mathrm{m} / \mathrm{z}=522.2191$, found $=523.2270[\mathrm{M}+\mathrm{H}]^{+} .{ }^{1} \mathrm{H}$ NMR $\left(400 \mathrm{MHz}, \mathrm{D}_{6}\right.$-DMSO) $\delta: 8.57(\mathrm{~d}, J=8.5 \mathrm{~Hz}, 1 \mathrm{H}), 8.51$ $(\mathrm{d}, J=4.9 \mathrm{~Hz}, 2 \mathrm{H}), 8.47(\mathrm{~s}, 1 \mathrm{H}), 8.08\left(\mathrm{td}, J_{1}=8.7 \mathrm{~Hz}, J_{2}=6.4 \mathrm{~Hz}, 1 \mathrm{H}\right), 7.59(\mathrm{~m}, 1 \mathrm{H}), 7.34$ $\left(\mathrm{td}, J_{1}=8.3 \mathrm{~Hz}, J_{2}=2.1 \mathrm{~Hz}, 1 \mathrm{H}\right), 7.18(\mathrm{t}, J=4.9 \mathrm{~Hz}, 1 \mathrm{H}), 7.16(\mathrm{~d}, J=3.0 \mathrm{~Hz}, 1 \mathrm{H}), 3.99(\mathrm{~m}$, $1 \mathrm{H}), 3.14(\mathrm{~d}, J=9.2 \mathrm{~Hz}, 1 \mathrm{H}), 2.99(\mathrm{~d}, J=11.2 \mathrm{~Hz}, 1 \mathrm{H}), 2.72\left(\mathrm{td}, J_{1}=11.0 \mathrm{~Hz}, J_{2}=3.6 \mathrm{~Hz}\right.$, $1 \mathrm{H}), 2.22(\mathrm{~m}, 2 \mathrm{H}), 2.11(\mathrm{t}, J=11.5 \mathrm{~Hz}, 1 \mathrm{H}), 2.01(\mathrm{t}, J=10.4 \mathrm{~Hz}, 1 \mathrm{H}), 1.83-1.87(\mathrm{~m}, 1 \mathrm{H})$, $1.61(\mathrm{~m}, J=3.8 \mathrm{~Hz}, 1 \mathrm{H}), 1.48-1.52(\mathrm{~m}, 1 \mathrm{H}), 1.34-1.39(\mathrm{~m}, 1 \mathrm{H}), 1.08(\mathrm{~m}, 2 \mathrm{H}), 0.82-0.88(\mathrm{~m}$, $1 \mathrm{H}), 0.46-0.51(\mathrm{~m}, 2 \mathrm{H}), 0.10(\mathrm{~m}, 2 \mathrm{H})$.

$(3 R, 4 S)-1-C y c l o p r o p y l m e t h y l-4-\{[5-(2,4-d i f l u o r o-p h e n y l)-i s o x a z o l e-3-c a r b o n y l]-a m i n o\}-$ piperidine-3-carboxylic acid (1-pyrimidin-2-yl-cyclopropyl)-amide (30) was synthesised by reductive amination of $(3 R, 4 S)-4-\{[5-(2,4$-difluoro-phenyl)-isoxazole-3-carbonyl $]$-amino $\}$ - 
piperidine-3-carboxylic acid (1-pyrimidin-2-yl-cyclopropyl)-amide hydrochloride with cyclopropanecarboxaldehyde. LC-MS method A: $\mathrm{tR}=0.81 \mathrm{~min} ;[\mathrm{M}+\mathrm{H}]+=523.31$. Chiral HPLC: $\mathrm{tR}=12.0 \mathrm{~min} ;>99 \%$ ee; column: ChiralPak IC 4.6x250 mm, $5 \mu \mathrm{m}$; detector wavelength: $254 \mathrm{~nm}$; eluent: 10\% heptane 0.05\% DEA; 90\% ethanol 0.05\% DEA; flow: 1.2 $\mathrm{mL} / \mathrm{min}$; BPR: 150 bar; temperature: $25^{\circ} \mathrm{C}$; injection volume: $6 \mu \mathrm{l}$.LC-HRMS: $\mathrm{t}_{\mathrm{R}}=0.62 \mathrm{~min}$; $\mathrm{m} / \mathrm{z}=522.2191$, found $=523.2273[\mathrm{M}+\mathrm{H}]^{+} .{ }^{1} \mathrm{H}$ NMR $\left(400 \mathrm{MHz}, \mathrm{D}_{6}-\mathrm{DMSO}\right) \delta: 9.38-9.47(\mathrm{~s}$, $1 \mathrm{H}), 8.65(\mathrm{~d}, J=4.7 \mathrm{~Hz}, 2 \mathrm{H}), 8.10-8.11(\mathrm{~m}, 1 \mathrm{H}), 8.04\left(\mathrm{td}, J_{1}=8.7 \mathrm{~Hz}, J_{2}=6.5 \mathrm{~Hz}, 1 \mathrm{H}\right)$, $7.58(\mathrm{~m}, 1 \mathrm{H}), 7.33\left(\mathrm{td}, J_{1}=8.6 \mathrm{~Hz}, J_{2}=2.2 \mathrm{~Hz}, 1 \mathrm{H}\right), 7.27(\mathrm{t}, J=4.7 \mathrm{~Hz}, 1 \mathrm{H}), 7.13(\mathrm{~d}, J=2.8$ $\mathrm{Hz}, 1 \mathrm{H}), 4.17-4.23$ (s, $1 \mathrm{H}), 3.11-3.24(\mathrm{~m}, 1 \mathrm{H}), 2.81-2.96(\mathrm{~m}, 1 \mathrm{H}), 2.73$ (m, $1 \mathrm{H}), 2.38-2.44$ (m, 1 H), 2.24-2.36 (m, 4 H), 1.81-1.91 (m, $1 \mathrm{H}), 1.46-1.58$ (m, $2 \mathrm{H}), 1.22-1.30(\mathrm{~m}, 2 \mathrm{H}), 0.87-$ 0.96 (m, $1 \mathrm{H}), 0.45-0.55$ (m, $2 \mathrm{H}), 0.08-0.18(\mathrm{~m}, 2 \mathrm{H})$.

(3S,4R)-1-Cyclopropylmethyl-4-\{[5-(2,4-difluoro-phenyl)-isoxazole-3-carbonyl]-amino\}piperidine-3-carboxylic acid (1-pyrimidin-2-yl-cyclopropyl)-amide (31) was synthesised by reductive amination of $(3 S, 4 R)-4-\{[5-(2,4$-difluoro-phenyl)-isoxazole-3-carbonyl $]$-amino $\}$ piperidine-3-carboxylic acid (1-pyrimidin-2-yl-cyclopropyl)-amide hydrochloride with cyclopropanecarboxaldehyde. LC-MS method $A: t_{R}=0.71 \mathrm{~min} ;[\mathrm{M}+\mathrm{H}]^{+}=523.18$. Chiral HPLC: $\mathrm{t}_{\mathrm{R}}=18.4 \mathrm{~min} ;>99 \%$ ee; column: ChiralPak IC 4.6x250 mm, $5 \mu \mathrm{m}$; detector wavelength: $254 \mathrm{~nm}$; eluent: 10\% heptane 0.05\% DEA; 90\% ethanol 0.05\% DEA; Flow: 1.2 $\mathrm{mL} / \mathrm{min}$; BPR: 150 bar; temperature: $25^{\circ} \mathrm{C}$; injection volume: $6 \mu \mathrm{l} . \mathrm{LC}-\mathrm{HRMS}: \mathrm{t}_{\mathrm{R}}=0.62 \mathrm{~min}$; $\mathrm{m} / \mathrm{z}=522.2191$, found $=523.2275[\mathrm{M}+\mathrm{H}]^{+} .{ }^{1} \mathrm{H}$ NMR $\left(400 \mathrm{MHz}, \mathrm{D}_{6}\right.$-DMSO) $\delta:$ 9.39-9.47 (s, $1 \mathrm{H}), 8.65(\mathrm{~d}, J=4.9 \mathrm{~Hz}, 2 \mathrm{H}), 8.10(\mathrm{~d}, J=7.8 \mathrm{~Hz}, 1 \mathrm{H}), 8.04\left(\mathrm{td}, J_{1}=8.7 \mathrm{~Hz}, J_{2}=6.5 \mathrm{~Hz}, 1\right.$ H), 7.55-7.61 (m, $1 \mathrm{H}), 7.33\left(\mathrm{td}, J_{1}=8.5 \mathrm{~Hz}, J_{2}=2.3 \mathrm{~Hz}, 1 \mathrm{H}\right), 7.26(\mathrm{t}, J=4.9 \mathrm{~Hz}, 1 \mathrm{H}), 7.12$ $(\mathrm{d}, J=2.9 \mathrm{~Hz}, 1 \mathrm{H}), 4.16-4.20($ broad s, $1 \mathrm{H}), 3.12-3.24($ broad s, $1 \mathrm{H}), 2.82-2.93(\mathrm{~m}, 1 \mathrm{H})$, $2.72(\mathrm{~d}, J=4.0 \mathrm{~Hz}, 1 \mathrm{H}), 2.37-2.42(\mathrm{~m}, 1 \mathrm{H}), 2.29-2.34(\mathrm{~m}, 2 \mathrm{H}), 2.24\left(\mathrm{dd}, J_{1}=6.5 \mathrm{~Hz}, J_{2}=\right.$ 
4.7 Hz, $2 \mathrm{H}), 1.82-1.91(\mathrm{~m}, 1 \mathrm{H}), 1.55-1.59(\mathrm{~m}, 1 \mathrm{H}), 1.48-1.52(\mathrm{~m}, 1 \mathrm{H}), 1.22-1.30(\mathrm{~m}, 2 \mathrm{H})$,

0.88-0.94 (m, $1 \mathrm{H}), 0.48$ (m, $2 \mathrm{H}), 0.12$ (d, $J=4.3 \mathrm{~Hz}, 2 \mathrm{H})$.

\section{hKV11.1 ("hERG") assay}

hKV11.1 ("hERG") assay. Compounds were evaluated for block of hKV11.1 channels using CHO cells stably expressing the hERG gene (bSys, Witterswil, Switzerland) and the QPatch platform (Sophion, Ballerup, Denmark). $\mathrm{K}+$ tail currents were measured at $-40 \mathrm{mV}$ following a $500 \mathrm{~ms}$ depolarization to $+20 \mathrm{mV}$ from a holding voltage of $-80 \mathrm{mV}$. The external solution contained $150 \mathrm{mM} \mathrm{Na}+, 4 \mathrm{mM} \mathrm{K}+, 1 \mathrm{mM} \mathrm{Mg} 2+$, and $1.2 \mathrm{mM} \mathrm{Ca} 2+$. Compound effects were quantified 3 min after application to the cells.

\section{In Vitro DMPK}

Inhibition of Cytochrome P450 Enzymes. The potential of the compounds for inhibition of the main human $\mathrm{P} 450$ isoforms, i.e., 2C9, 2D6, and 3A4, was evaluated using human liver microsomes and specific marker reactions for each enzyme. Diclofenac 4'-hydroxylation was used for CYP2C9, dextromethorphan $O$-demethylation was used for CYP2D6 and midazolam1'-hydroxylation and testosterone 6 6 -hydroxylation were used for CYP3A4. Experiments were performed around the respective $K \mathrm{~m}$ values of the marker substrates, and metabolite formation was monitored by LC-MS/MS. Inhibitor concentrations up to $50 \mu \mathrm{M}$ were added, and the performance of the assay was controlled by the use of specific inhibitors for each P450 isoform.

Metabolic Stability in Liver Microsomes. Incubation with human, rat and dog liver microsomes were performed to assess metabolic stability at a single substrate concentration of $1 \mu \mathrm{M}$. A $1 \mu \mathrm{L}$-aliquot of the compounds stock solutions in DMSO were added to $100 \mathrm{mM}$ phosphate buffer ( $\mathrm{pH} 7.4$ ) containing the liver microsomes at a concentration of $0.5 \mathrm{mg} / \mathrm{mL}$ 
and the mixture was incubated at $37^{\circ} \mathrm{C}$ in an Eppendorf thermomixer at $450 \mathrm{rpm}$. The reaction was initiated by addition of $100 \mu \mathrm{L}$ of NADPH-regenerating system containing the glucose-6phosphate dehydrogenase and at the pre-defined time points, $0,2.5,5,10,15,20,30$ and 45 $\min , 100 \mu \mathrm{L}$ of the incubation was transferred in $100 \mu \mathrm{L}$ of ice-cold methanol to stop the reaction. Samples were centrifuged at $3220 \mathrm{~g}$ for $20 \mathrm{~min}$ at $4{ }^{\circ} \mathrm{C}$ and the supernatants were submitted to LC/MS-MS analysis.

Plasma Protein Binding. The binding to plasma proteins was determined by equilibrium analysis using a Pierce rapid equilibrium dialysis (RED) device (Thermo Fisher Scientific, Reinach, Switzerland) and incubating on a shaker at $37^{\circ} \mathrm{C}$ for $4 \mathrm{~h}$. The device was comprised of two compartments (protein compartment and buffer compartment) separated by a dialysis membrane with a molecular weight cut-off of $8 \mathrm{kDa}$, which allowed unbound test compound but not proteins, to traverse the membrane and equilibrate between the two compartments. The donor compartment was either human, rat or dog plasma. Donor and receiver (containing phosphate buffer, $\mathrm{pH}$ 7.4) were analyzed by LC-MS/MS.

\section{In Vivo DMPK}

Pharmacokinetic Studies in Rat and Dog. The amorphous hydrochloric salt of ACT-10041239 was used. Male Wistar rats with a body weight of ca. 200-250 g were used for pharmacokinetic experiments. For intravenous sampling, a jugular vein catheter was implanted 2 days prior to drug dosing under aseptic conditions. After recovery from general isoflurane anesthesia, animals were housed individually with free access to water and food during the recovery period and the entire duration of the experiment. For intravenous use the compound was formulated as an aqueous solution in 5\% mannitol and water and for oral gavage as a solution in purified water. For both formulations, $\mathrm{pH}$ was adjusted to a value of 5.2 and 3.6, respectively with an $\mathrm{NaOH}$ solution. Male Beagle dogs with body weights of $13.4-16.0 \mathrm{~kg}$ at 
the start of treatment were used in a crossover design with a washout period of 7 days. All experiments were performed in fasted state, and gastric $\mathrm{pH}$ was controlled by giving intramuscular pentagastrin at a dose of $6 \mathrm{mg} / \mathrm{kg} 30$ minutes after oral dosing For intravenous use the compound was formulated as an aqueous solution in $5 \%$ mannitol and water and for oral gavage as a solution in purified water and $\mathrm{pH}$ was adjusted to a value of 5.0 in both cases with an $\mathrm{NaOH}$ solution. Serial blood samples of $0.25 \mathrm{ml}$ (rats) or $2 \mathrm{ml}$ (dogs) were taken over a period of 24 hours and transferred into vials fortified with EDTA as anticoagulant. Blood samples after oral dosing to rats were taken under light isoflurane anesthesia. Plasma was generated by centrifugation and stored at $-20^{\circ} \mathrm{C}$ pending analysis. Analysis was performed using liquid chromatography coupled to mass spectrometry (LC-MS-MS) after protein precipitation with methanol and centrifugation at $3220 \mathrm{~g}$ for $20 \mathrm{~min}$ at $4^{\circ} \mathrm{C}$. Pharmacokinetic parameters were estimated with the WinNonlin software (Pharsight Corporation, Mountain View, CA, USA) using non-compartmental analysis.

\section{Physico-Chemical Properties Measurement}

Log D7.4 determination. The distribution coefficient $\log _{7.4}$ of a compound was determined by a miniaturized shake-flask method in screening mode. After overnight equilibration at room temperature in the 1-octanol/phosphate buffer saline $\mathrm{pH} 7.4$ system, the $\operatorname{LogD}_{7.4}$ was calculated from the HPLC peak area ratio of both phases without $\mathrm{pH}$ control. $\log \mathrm{D}$ values higher than 4.1 were reported as $>4.1$ due to HPLC detection limit.

pKa determination. The ionization constant $\mathrm{pKa}$ of a compound was determined using SiriusT3 instrument and software from Pion, Inc. Measurement was done by potentiometric or spectroscopic titration, depending on chromophore, from $\mathrm{pH} 2$ to 12 or from $\mathrm{pH} 12$ to 2 , at $25^{\circ} \mathrm{C}$ or $37^{\circ} \mathrm{C}$ in a $0.1 \mathrm{M} \mathrm{KCl}$ solution at concentrations respectively between $0.7 \mathrm{mM}$ to $2 \mathrm{mM}$, introduced as powder, and $0.01 \mathrm{mM}$ to $0.1 \mathrm{mM}$, introduced as $2 \mu \mathrm{L}$ to $5 \mu \mathrm{L}$ DMSO stock 
solution of $10 \mathrm{mM}$ to $30 \mathrm{mM}$. During titration, solution was stirred using a paddle under nitrogen flow at the liquid surface. In case of precipitation, the use of a co-solvent may have been needed.

Solubility determination. The solubility of a compound was determined by the screening solubility assay. Starting material was a $10 \mathrm{mM}$ DMSO stock solution, from which the solvent was evaporated and then the medium of interest was added. The approximate solubility (in $\mu \mathrm{g} / \mathrm{mL}$ ) up to around $1000 \mu \mathrm{g} / \mathrm{mL}$ (depending on molar mass) of a compound was determined in the medium of interest after $24 \mathrm{~h}$ of equilibration at $25^{\circ} \mathrm{C}$ and filtration of the possible solid remaining. Solubility was determined in the following medium: FaSSIF-V1, FeSSIF-V1, phosphate buffer saline $\mathrm{pH} 7.4$ or phosphate buffer $\mathrm{pH} 7$.

Differential scanning calorimetry (DSC). DSC data were collected on a Mettler Toledo STARe System (DSC822e module, measuring cell with ceramic censor and STAR software version 9.20) equipped with a 34-position auto-sampler. The instrument was calibrated for energy and temperature using certified indium. Typically, 1-5 $\mathrm{mg}$ of each sample, in an automatically pierced aluminium pan, was heated at $10{ }^{\circ} \mathrm{C} \mathrm{min}^{-1}$, unless stated otherwise, from $-20^{\circ} \mathrm{C}$ to $280^{\circ} \mathrm{C}$. A nitrogen purge at $20 \mathrm{~mL} \mathrm{~min}^{-1}$ was maintained over the sample. Peak temperatures were reported for melting points.

\section{Single Crystal X-ray Structure Analysis of ACT-1004-1239 (28f)}

\section{Data collection and refinement statistics}

Crystals of $40\left(\mathrm{C}_{27} \mathrm{H}_{28} \mathrm{~F}_{2} \mathrm{~N}_{6} \mathrm{O}_{3} \bullet \mathrm{MeOH}\right)$ formed in the orthorhombic chiral space group $\mathrm{P} 2{ }_{1} 2_{1} 2_{1}$. A total of 3644 reflections were collected at $253 \mathrm{~K}$. Molecules/unit cell $\mathrm{Z}=2$, cell dimensions $\mathrm{a}=4.8267(1) \AA, \mathrm{b}=21.2971(6) \AA, \mathrm{c}=26.260(8) \AA ; \mathrm{V}=2699.4(1) \AA^{3}$; calculated density $=1.286 \mathrm{~g} \mathrm{~cm}^{-3}$. The final R-factor of $4.83 \%$ was obtained for 2177 observed reflections 
$(\mathrm{I}>4 \sigma(\mathrm{I}))$ for a resolution of $1.05 \AA$; largest difference peak and hole were 0.34 and $-0.28 \mathrm{e}^{-}$ $\AA^{-3}$, respectively. Crystallographic file has been deposited under the number CCDC 1993933.

The terminal cyclo-propyl ring is disordered over two positions. With a free variable and fixed Uiso both partitions refine to a 52/48 ratio. In the final refinement they were fixed to 50/50 with EADP for each partition. They were left isotropic. If they are refined anisotropic, R1 goes down to app. $3.5 \%$, but the shifts of the atoms are big and they doesn't remain stable. To avoid introduction of constrains for the thermal parameters, these disordered positions were refined isotropic to achieve displacement shifts to be zero.

There is a $\mathrm{MeOH}$ which is disordered over at least 3 positions. Due to the "not so good" data quality, the solvate positions can't be fully refined, nor found completely. Therefore, to avoid such problems and instability of the refinement, the MeOH solvate was SQUEEZED out.

\section{Biological Assays In Vitro}

CXCR7-agonist 1 assay: CHO-K1 CXCR7 $\beta$-arrestin cells were detached from culture dishes with a cell dissociation buffer (Invitrogen, Catalogue \#13151-014) and collected in their culture medium. Cells (5000 per well in $20 \mu \mathrm{L}$ ) were seeded in 384-well plates (Greiner, Catalogue \#781098). The plates were incubated at $37^{\circ} \mathrm{C} / 5 \% \mathrm{CO}_{2}$ for $24 \mathrm{~h}$. The medium of each well was replaced with $20 \mu \mathrm{L}$ of OPTIMEM (Invitrogen, Catalogue \#31985) for 3-4 h. Test compounds were dissolved at a concentration of $10 \mathrm{mM}$ in DMSO and either tested at $10 \mathrm{uM}$ or serially diluted in DMSO to $200 \mathrm{X}$ of the final concentration to be used for dose-response testing. Compounds were then further diluted 1:33.3 in 1X HBSS. The diluted compounds ( $5 \mu \mathrm{L} /$ well) were added to the assay plates and incubated for 15 minutes or $3 \mathrm{~h}$ at $37^{\circ} \mathrm{C}$. Next, the agonist 1 was diluted in HBSS/20mM HEPES/0.2\% BSA to $6 \mathrm{X}$ of the final concentration to be used (the final concentration used was $5 \mathrm{nM}$, equivalent to its $\mathrm{EC}_{80}$ value) and $5 \mu \mathrm{L} /$ well was added to the assay plate. The effect of each compound was calculated as a percentage of the maximum 
assay signal. The mean minimum and maximum values were obtained from control wells in the same plate and were set at $0 \%$ and $100 \%$.

Insurmountability assay: For the insurmountability assay, serial dilution of CXCL12 or CXCL11 were used instead of 1 . The plates were incubated for another 90 minutes at $37^{\circ} \mathrm{C}$. The detection reagent (12 $\mu \mathrm{L}$; Detection Kit, DiscoveRx, \#93-0001) was then added to the wells and the plates were incubated for $1 \mathrm{~h}$ at room temperature. The resulting luminescent signal was read in a microplate reader (Envision, Perkin Elmer). Of note, the calculated $\mathrm{IC}_{50}$ values fluctuated depending on the daily performance of the cellular assay.

CXCR7-CXCL12 assay: CXCR7-bla U2OS cells were detached from culture dishes with 0.05\% trypsin-EDTA and collected in growing medium (McCoy's 5A 90\% (v/v), dialyzed fetal calf serum (FCS) $10 \%(\mathrm{v} / \mathrm{v}), 0.1 \mathrm{mM}$ non-essential amino acids (NEAA), 25mM HEPES

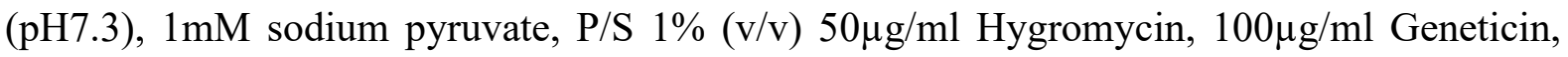
$200 \mu \mathrm{g} / \mathrm{ml}$ Zeocin), spinned down and resuspended in assay medium (McCoy’s 5A 90\% (v/v), dialyzed FCS 1\% (v/v), 0.1mM NEAA, 25mM HEPES (pH7.3), P/S 1\% (v/v)). 10’000 cells per well (in $30 \mu \mathrm{l})$ were seeded in a 384 well plate (black-walled, clear bottom). The plates were incubated at $37^{\circ} \mathrm{C} / 5 \% \mathrm{CO}_{2}$ for 24 hours. Test compounds were dissolved to $10 \mathrm{mM}$ in DMSO and serially diluted in DMSO to $500 \mathrm{X}$ of the final concentration for dose response curves. Compounds were then diluted 1:100 in assay medium to $5 \mathrm{X}$ of the final concentration. $10 \mu \mathrm{l} /$ well of diluted compounds were added to the assay plate and incubated for 15 minutes at $37^{\circ} \mathrm{C}$. Thereafter $\mathrm{CXCL} 12 \alpha$ was diluted in assay medium to $5 \mathrm{X}$ of the final concentration (Final concentration: $30 \mathrm{nM}$ ). This corresponds to its EC80 value for receptor activation) and $10 \mu \mathrm{l} /$ well were added to the assay plate. The plate was incubated for $22 \mathrm{hrs}$ at $37^{\circ} \mathrm{C} .10 \mu \mathrm{l} / \mathrm{well}$ of detection reagent (LiveBLAzerTM-FRET B/G (CCF4-AM) substrate) was transferred to the assay plate and the plate was incubated for 2 hours at room temperature protected from light. Fluorescent counts were determined (Scan1: Ex 409/20nm, Em 460/30nm, Scan 2: Ex 
409/20nm, Em 530/30nm). The calculated emission ratio was used for IC50 determination. The effect of each compound was calculated as a percentage of the maximum assay signal. The mean minimum and maximum values were obtained from control wells in the same plate and were set at $0 \%$ and $100 \%$. The calculated IC50 values may fluctuate depending on the daily cellular assay performance.

Determination of the Ki, Kon and Koff constants: HEK-SNAP.hCXCR7.NLuc cells were washed once with PBS and 100 nM SNAP Lumi4-Tb (Cisbio, Catalogue \#SSNPTBC) prepared in 1x Tag-lite ${ }^{\circledR}$ buffer (TLB, Cisbio, Catalogue \#LABMED) was added and further incubated for $1 \mathrm{~h}$ at $37^{\circ} \mathrm{C}, 5 \% \mathrm{CO}_{2}$. Excess SNAP-Lumi4-Tb was removed by washing cells 3 times with TLB 1x. Cells were detached from culture dishes with cell dissociation buffer (Gibco Catalogue \#13151), resuspended in TLB and counted. Cells were then centrifuged at 200xg for $5 \mathrm{~min}$, the supernatant was removed by aspiration and cell pellets were resuspended in $1 \mathrm{x}$ TLB at a density of 0.4 million cells/mL. Cells were gently mixed by pipetting up and down several times. Cells were frozen in FCS 90\%/DMSO 10\% as ready-to-use labeled cells. The day of the assay, cells were thawed, resuspended in $10 \mathrm{~mL}$ TLB $1 \mathrm{x}$ and centrifuged for 5 min at 200xg. The supernatant was removed by aspiration and cell pellets were resuspended in TLB $1 \mathrm{x}$ at 0.4 million cells $/ \mathrm{mL}$. Cells were gently mixed by pipetting up and down several times. ACT-1004-1239A was dissolved to $10 \mathrm{mM}$ in DMSO and serially diluted in DMSO to 400x of the final concentration for dose-response curves. Compounds were further diluted 1/100 in TLB to $4 \mathrm{x}$ of the final concentration. $5 \mu \mathrm{L}$ of this solution were transferred to a white low volume plate, where $5 \mu \mathrm{L}$ of labeled ligand (3 nM CXCL12 AF647 diluted in TLB 1x to obtain a 4-fold concentrated solution) and $10 \mu \mathrm{L}$ of cells (4000 cells per well) were added. The final percentage of DMSO in each well was $0.25 \%$. The binding kinetics were measured immediately on a Pherastar FSX (measurement began 30 seconds after addition of the cells) every 30 seconds for $3 \mathrm{~h}$ at room temperature. 
CXCR7 assay using CXCR7 from various species: we established stable in vitro systems where CXCR7 from different species and human $\beta$-arrestin were overexpressed either in Chinese hamster ovary (CHO), U2OS or human embryonic kidney cells 293 (HEK293) cell lines (thereafter the " $\beta$-Arrestin CXCR7 cell lines"). In these systems, cells are engineered to co-express a ProLink ${ }^{\mathrm{TM}}(\mathrm{PK})$ tagged CXCR7 and an Enzyme Acceptor (EA) tagged $\beta$-arrestin (Table S1).

Table S1: List of the CXCR7 $\beta$-arrestin stable cell lines

\begin{tabular}{|l|c|c|}
\hline $\boldsymbol{\beta}$-Arrestin CXCR7 cell lines & Medium & Idorsia cell Bank number \\
\hline CHO-K1 humanCXCR7 $\beta$-arrestin & Ham's F12 & CC548 \\
\hline HEK293 ratCXCR7 $\beta$-arrestin clone\#2 & DMEM & CC423 \\
\hline CHO-K1 guinea pigCXCR7 $\beta$-arrestin clone\#41 & Ham's F12 & CC422 \\
\hline CHO-K1 macaqueCXCR7 $\beta$-arrestin clone\#48 & Ham's F12 & CC420 \\
\hline CHO-K1 dogCXCR7 $\beta$-arrestin clone\#12 & Ham's F12 & CC421 \\
\hline U2OS mouseCXCR7 $\beta$-arrestin & MEM & CC549 \\
\hline
\end{tabular}

The $\beta$-arrestin CXCR7 cell lines were grown to near confluency in the indicated medium (Table S1) supplemented with $10 \%$ fetal calf serum containing penicillin and streptomycin (100 units/mL each) under standard mammalian cell culture conditions at $37^{\circ} \mathrm{C}$ in a humidified atmosphere of $5 \% \mathrm{CO}_{2}$. Cells were detached from culture dishes with a cell dissociation buffer (Invitrogen, Catalogue \#13151-014) and collected in their growing medium. Cells (5000 cells per well in $20 \mu \mathrm{L}$ for all cell lines except HEK293 ratCXCR7, which were seeded at 20,000 cells per well in $20 \mathrm{uL}$ ) were seeded in a 384 well plate (Greiner, Catalogue \#781098). HEK293 ratCXCR7 cells were seeded on poly-L-Lysine (Cultrex, Catalogue \# 3438-100-01)-coated 384 well plates. The plates were incubated at $37^{\circ} \mathrm{C} / 5 \% \mathrm{CO}_{2}$ for $24 \mathrm{~h}$. The medium was replaced with $20 \mu \mathrm{L}$ OPTIMEM (Invitrogen Catalogue \#31985) for 3 to 5 h. ACT-1004-1239 was dissolved at a concentration of $10 \mathrm{mM}$ in DMSO and serially diluted in DMSO to 200X of the final concentration to be used for the dose response experiments. ACT-1004-1239 was then diluted 1:33.3 in Hank's balanced salt solution (HBSS) 1X. Diluted compounds ( $5 \mu \mathrm{L} /$ well) were added to the assay plates and incubated for $15 \mathrm{~min}$ at $37^{\circ} \mathrm{C}$. Next, CXCL12 (Peprotech, 
Catalogue \# 300-28A) was diluted in HBSS/20 mM HEPES/0.2\% BSA (Sigma, Catalogue\# A7030) to $6 \mathrm{X}$ of the final concentration (its $\mathrm{EC}_{80}$ value) and $5 \mu \mathrm{L} /$ well was added to the assay plates. The plates were incubated for $90 \mathrm{~min}$ at $37^{\circ} \mathrm{C}$. The detection reagent $(12 \mu \mathrm{L}$; Detection Kit, DiscoveRx, \#93-0001) was transferred to the assay plate and the plate was incubated for 1 $\mathrm{h}$ at room temperature. The luminescent signal was read in a microplate reader (Envision, Perkin Elmer).

Human CXCR4 intracellular calcium liberation (FLIPR) Assay: Molt4 cells were grown to near confluency in Roswell Park Memorial Institute (RPMI) medium supplemented with $10 \%$ fetal calf serum containing penicillin and streptomycin (100 units $/ \mathrm{mL}$ each) under standard mammalian cell culture conditions at $37{ }^{\circ} \mathrm{C}$ in a humidified atmosphere of $5 \% \mathrm{CO}_{2}$. Cells were centrifuged and resuspended in Dye Buffer (1X HBSS, 0.0375\% $\mathrm{NaHCO}_{3}, 20 \mathrm{mM}$ HEPES, 5.25 $\mathrm{mM}$ probenecid, $10 \mathrm{nM}$ Fluoro-4). The cells were incubated for $45 \mathrm{~min}$ at $37^{\circ} \mathrm{C}$. Cells were then washed 2 times with Wash Buffer (1X HBSS, 0.0375\% $\mathrm{NaHCO}_{3}, 20 \mathrm{mM}$ HEPES, $2.5 \mathrm{mM}$ probenecid, 0.1\% BSA), resuspended in Wash Buffer and 50000 cells in 50 $\mu \mathrm{L}$ per well, were seeded onto a 384-well clear-bottom black assay plate (Greiner), and sedimented by centrifugation. Stock solutions of ACT-1004-1239 were made up at a concentration of $10 \mathrm{mM}$ in DMSO and serially diluted in assay buffer to concentrations required for dose-response curves. CXCL12 (Peprotech, Catalogue \# 300-28A) was used as an agonist. A FLIPRII instrument (Molecular Devices) was operated following the manufacturer's instructions. ACT-1004-1239 $(10 \mu \mathrm{L})$ was added to each well and incubated for 20 min. Cells were activated by a final concentration of $17.5 \mathrm{nM}$ CXCL12 dissolved in the wash buffer. Fluorescence emission was recorded during test compound and CXCL12 addition, and emission peak values above base level after CXCL12 addition were exported. Values were normalized to high-level control (no antagonist added) after subtraction of baseline value control (no CXCL12 added). 


\section{Biological Assay In Vivo}

Target engagement in vivo: Male DBA/1 mice (body weight at study start: $22-27 \mathrm{~g}$ ) were purchased from Janvier Laboratories (Le Genest-Saint-Isle, France) and allowed to acclimatize for at least 7 days before use. All animals were housed in climate-controlled conditions within a $12 \mathrm{~h} \mathrm{light/dark} \mathrm{cycle} \mathrm{and} \mathrm{had} \mathrm{free} \mathrm{access} \mathrm{to} \mathrm{normal} \mathrm{chow} \mathrm{and} \mathrm{drinking} \mathrm{water,} \mathrm{in} \mathrm{accordance}$ with the guidelines of the Swiss Animal Protection Law. All animal experiments were carried out in accordance with the Swiss animal protection law, under protocols approved by the Basel Cantonal Veterinary Office.

The compound was formulated in $20 \%$ volume of Solutol HS15 and in $80 \%$ volume of $0.25 \%$ (w/w) methylcellulose/water. Oral single doses of ACT-1004-1239 (1, 10, 30 or $100 \mathrm{mg} / \mathrm{kg})$ or vehicle were given to healthy DBA/1 mice p.o. $(n=3-5$ per time point $)$ in a volume of $5 \mathrm{~mL} / \mathrm{kg}$. Blood samples were collected $0.5,6$ and $24 \mathrm{~h}$ post-administration of a single oral dose. Blood was centrifuged $\left(20,000 \mathrm{~g}, 5\right.$ minutes at $\left.4{ }^{\circ} \mathrm{C}\right)$ to prepare plasma samples and concentrations of CXCL12 were measured using a commercial mouse CXCL12/SDF1 $\alpha$ Quantikine enzyme-linked immunosorbent assay kit (R\&D Systems, catalog no. MCX120) according to manufacturer's instructions. All data are presented as mean + SEM. Statistical analysis were performed by One-way ANOVA followed by Dunnett's multiple comparisons test, using Graphpad Prism software (version 8). 


\section{References}

1. Aissaoui,A.; Guerry, P.; Lehembre, F.; Pothier, J.; Pouzol, L.; Richard-Bildstein, S.; Yuan, S.; Piperidine CXCR7 Receptor Modulators, World Patent WO 2018/019929, 2018.

2. Linder, J; Garner, T. P.; Williams, H. E. L.; Searle, M. S.; Moody, C. J. Telomestatin: Formal Total Synthesis and Cation-Mediated Interaction of Its seco-Derivatives with G-Quadruplexes. J. Am. Chem. Soc. 2011, 133, 1044-1051. 


\section{LC-MS traces of compounds 2-31}

The LC-MS methods used correspond to the descriptions in the chemistry part.

Compound 2:

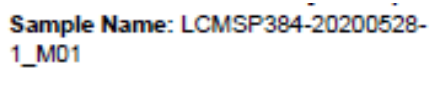

Expression

Auto-Comments

Auto-Summary

Purity

Target Mass

Target RT

บM:12216]

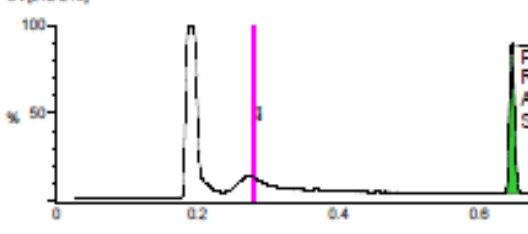

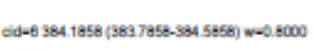

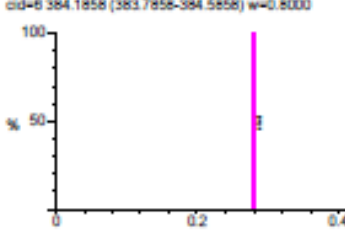

odd-11 962.1702 (981.7700-362.5706) w-0.8000

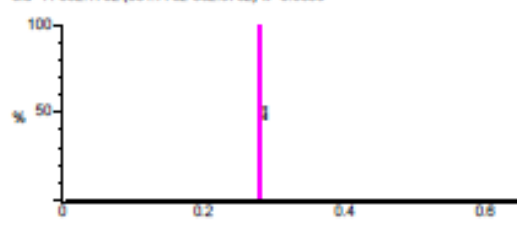

ACT No: ACT-642841

Instrument: ICHALW-DLO0021-SQD

ELN Nr: ELN148-0684.1

Acquisition time: 5/28/2020 8:49 AM

Result

GOOD

100.0000

384.3000

0.6460

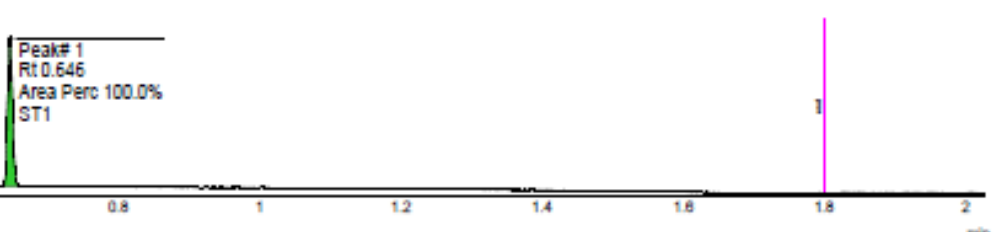

UV214 [4.4EOS ]

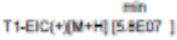

Poak 1 go. $845 \mathrm{~min}$ Ms(+) Es

3.6E07 onte

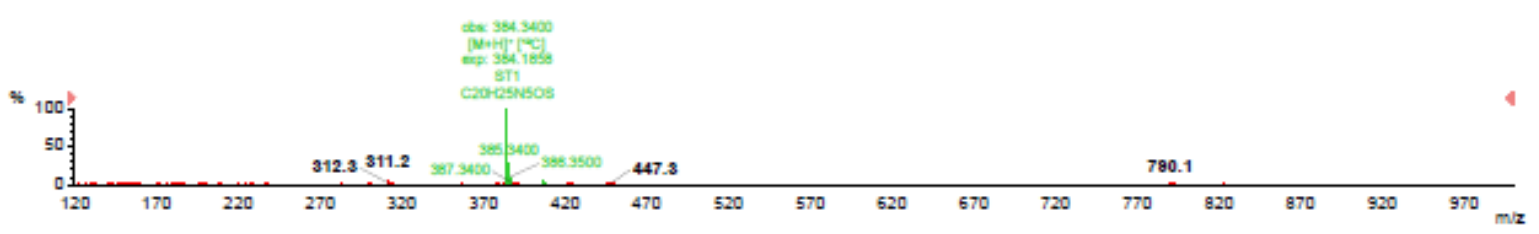


Compound 3:
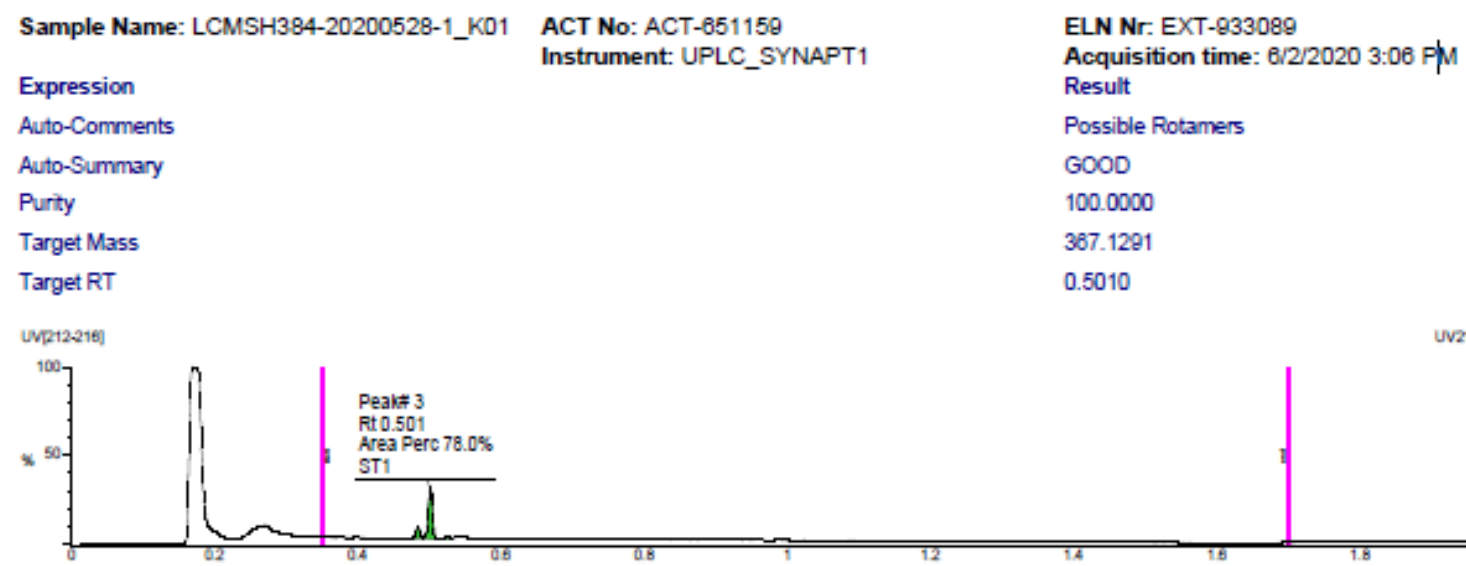

UV214 [0:3EOB ]

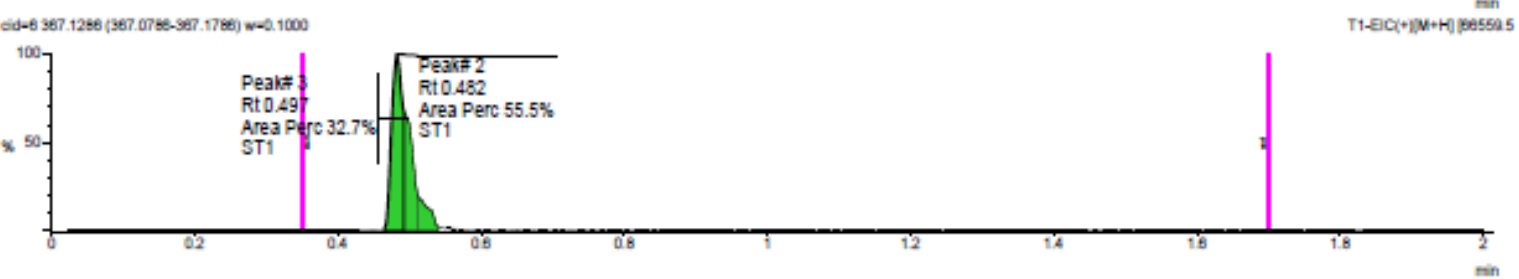

Poak $2 \mathrm{~g} 0.482 \mathrm{~min}$ M $3(+)$ Es

62807.8 onte

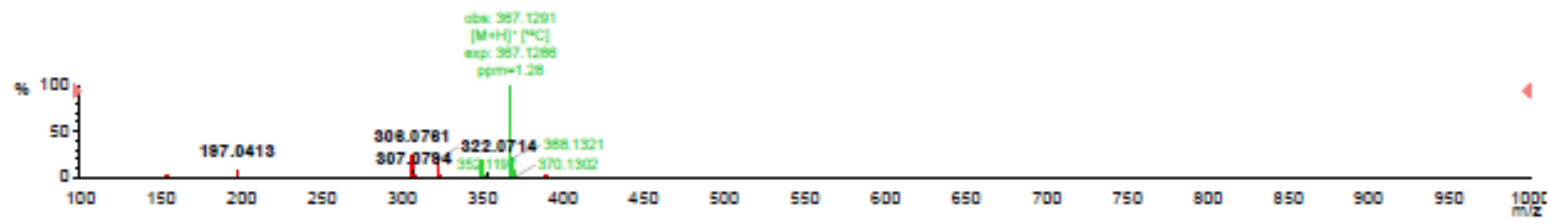

Poak $390.600 \mathrm{~min}$ Mos(+) Es

62810.4 onte
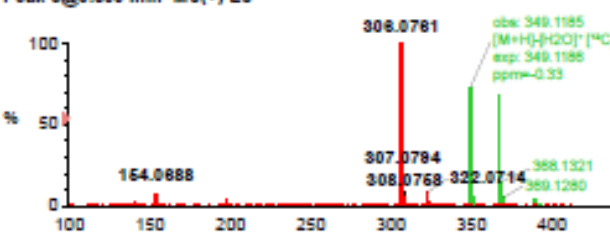

Poak 4 geo.617 min $M 3(+)$ Es

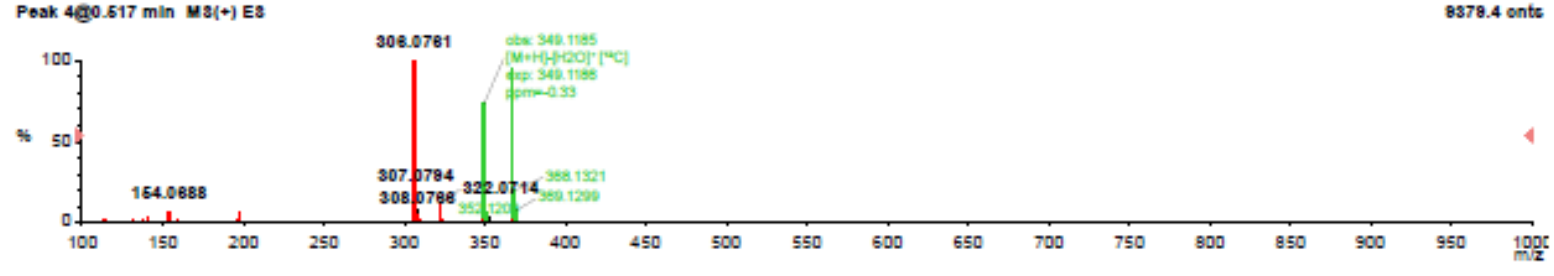


Compound 4:

Sample Name: LCMSH384-20200609-

1_MO5

Expression

Auto-Comments

Auto-Summary

Purity

Target Mass

Target RT

[พเ212.216]
ACT No: ACT- 655552

Instrument:

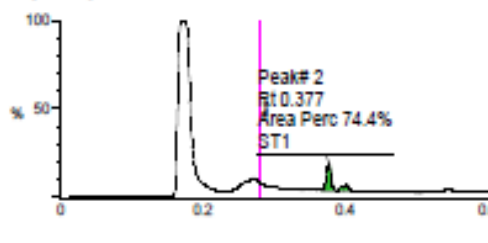

eid-6 327.1515 (\$27. 1015-327. 2015) w-0.1000

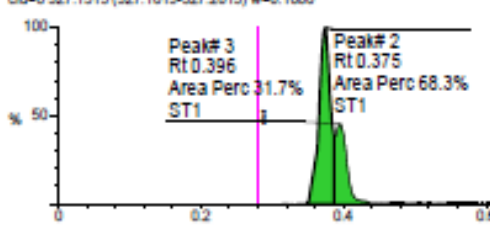

ELN Nr: ELN098-0668_A03

Acquisition time: $6 / 12 / 2020$ 11:26 AM

Result

Possible Rotamers

GOOD

100.0000

327.1524

0.3770

UV214 [82EOE ]

Poak 2 ggo.376 min Ma(+) Es

78080.0 onte
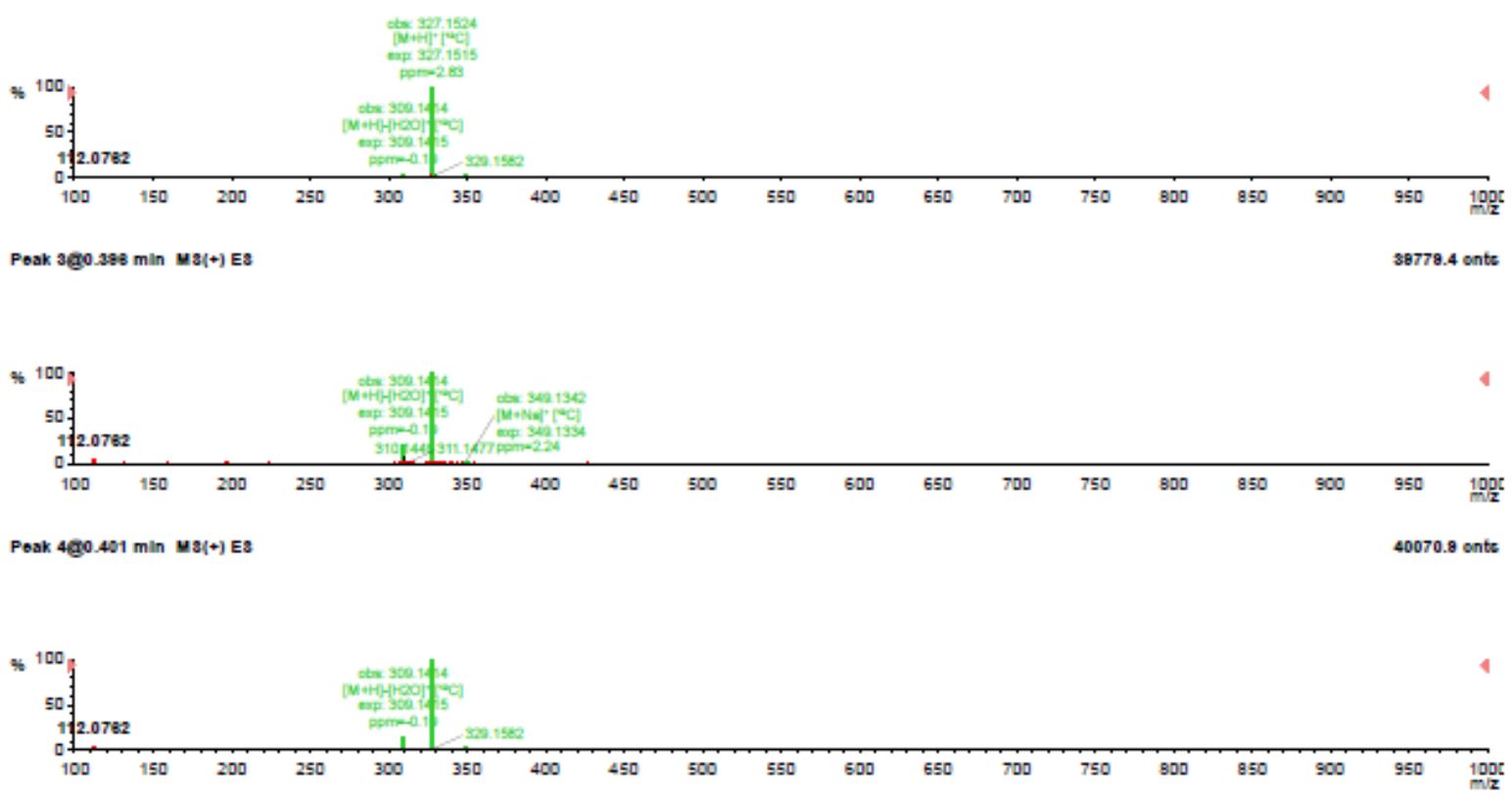
Compound 5:
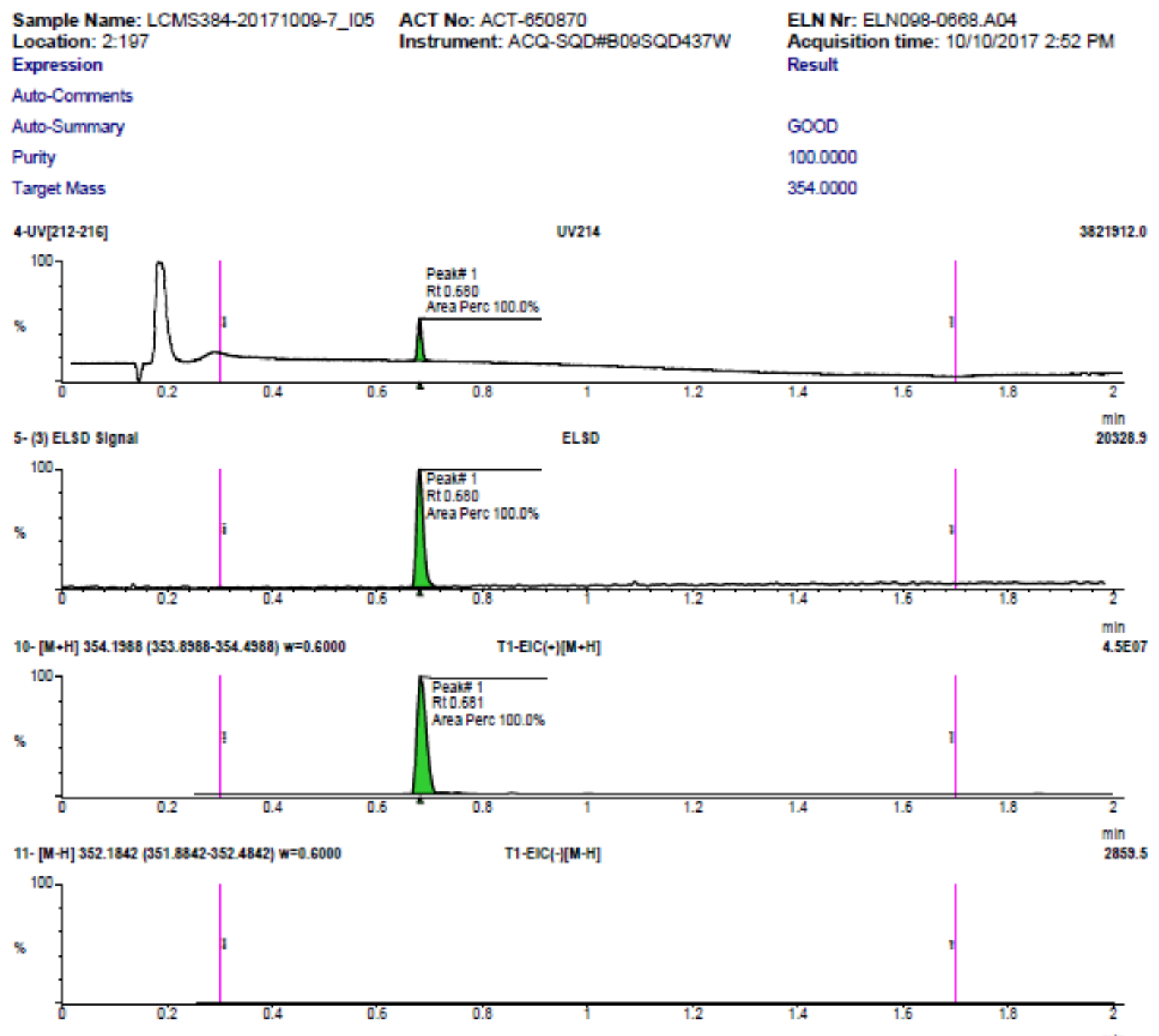

Poak $190.881 \mathrm{~min} M s(+) \mathrm{Es}$

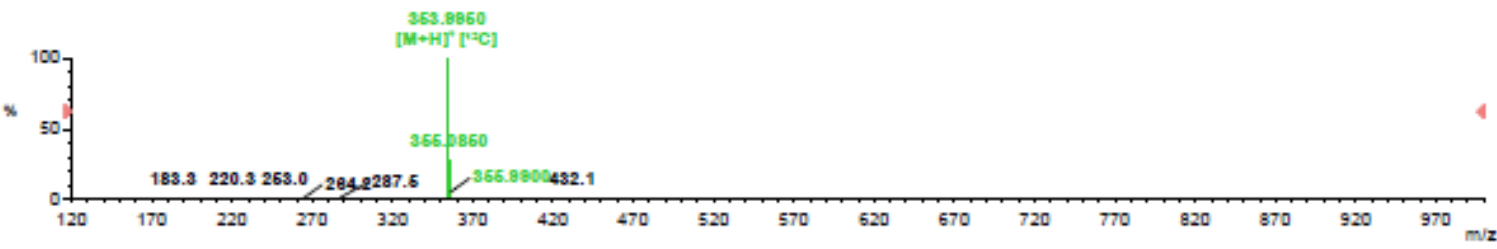


Compound 6:

Sample Name: LCMS384-20200624-1_M01 ACT No: ACT-582817

Instrument: ICHALW-DL00021-SQD

\section{Expression}

Auto-Comments

Auto-Summary

Purity

Target Mass

Target RT

uv[212216]

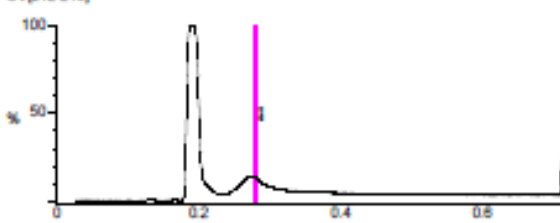

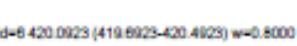

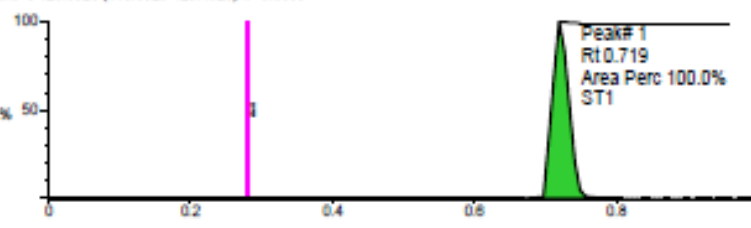

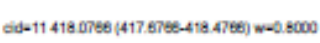

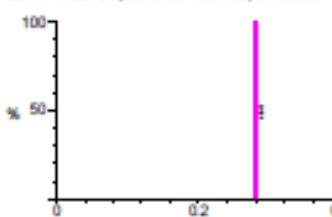

ELN Nr: ELN186-4537.1

Acquisition time: 6/24/2020 2-44 PM Result

GOOD

98.1000

420.3000

0.7180

UV214 [4.3EOS ]

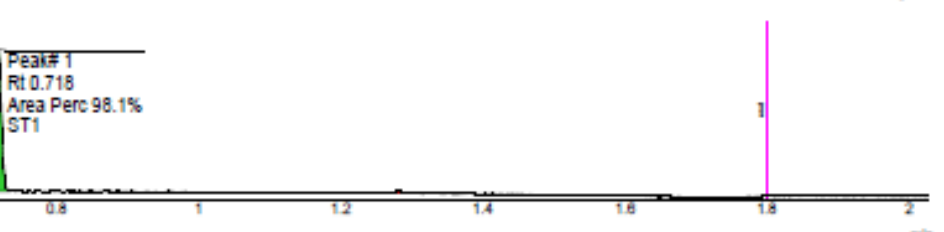

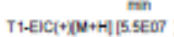

Poak 1 ge. 719 min us/+) Es

3.3E07 onte

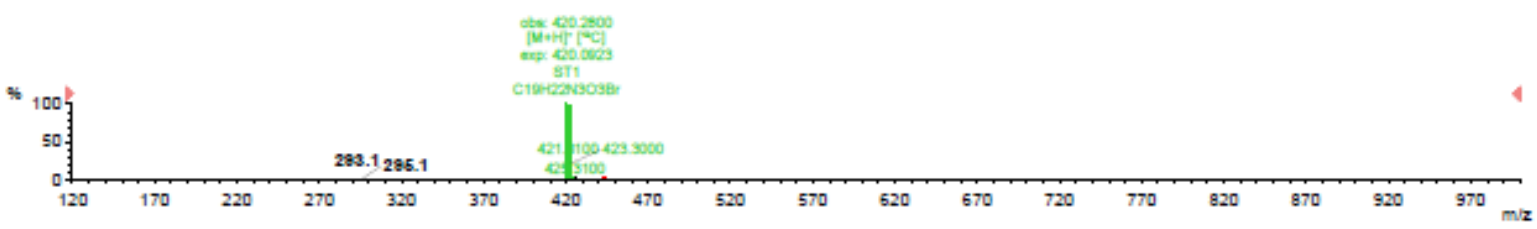


Compound 7a:

3: UV Detector: 212_216

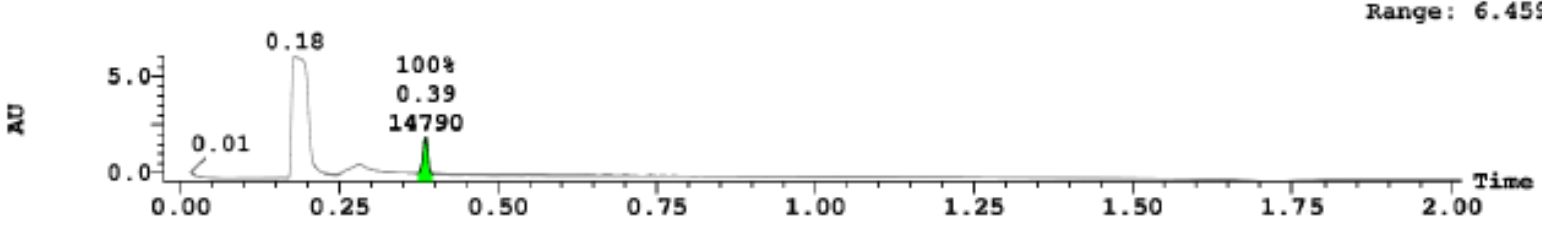

1: MS ESt :285.141 1.0000Da

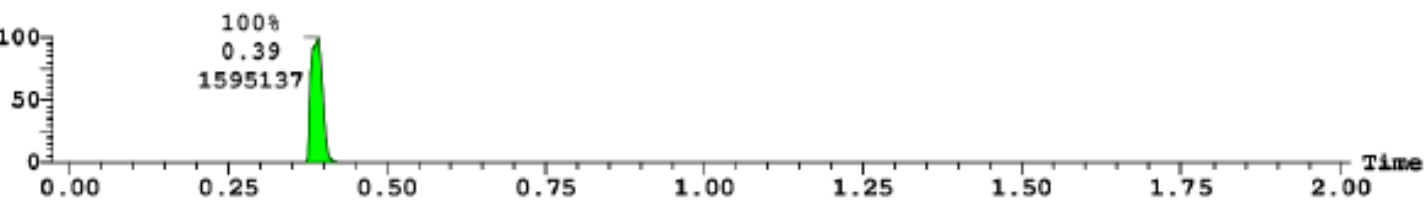

2: MS ES- :283.126 1.0000Da

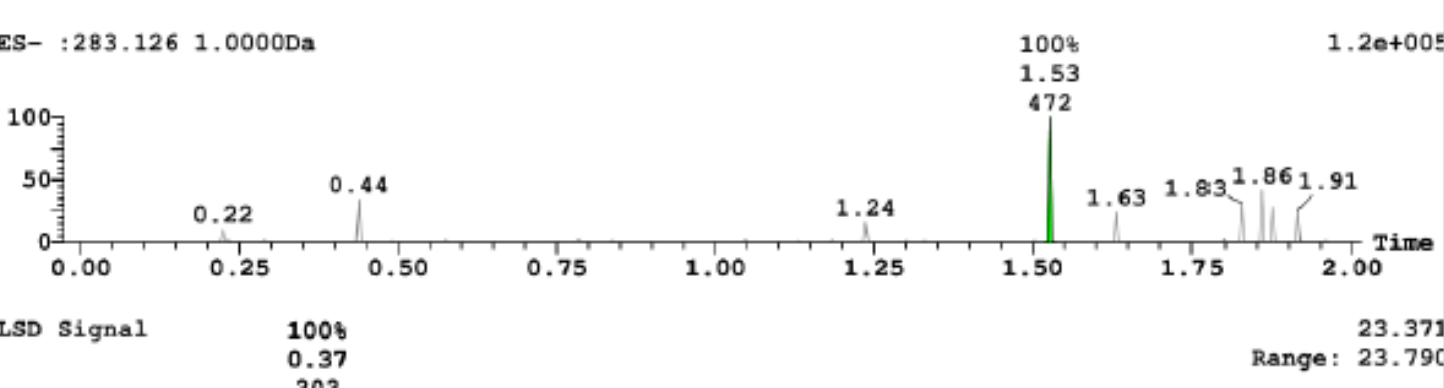

(1) ELSD Signal 1008

号

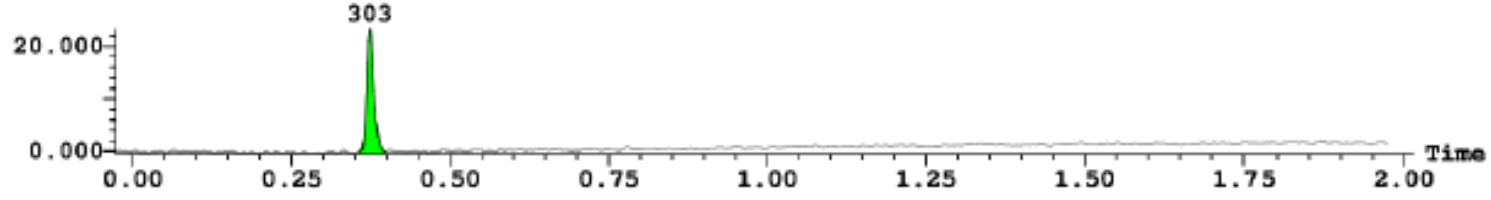

2: (Time: 0.39$)$ Combine (44:49-(24:26+66:69))

285.2

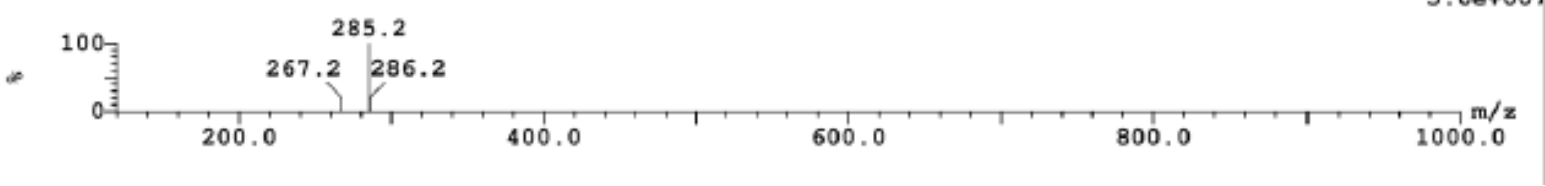


Compound 7b:

Sample Name: LCMSP384-20200528-1_K07 ACT No: ACT-707103

Instrument: ICHALW-DL00021-SQD

Auto-Comments

Auto-Summary

Purity

Target Mass

Target RT

UVI212.216

\section{Expression}

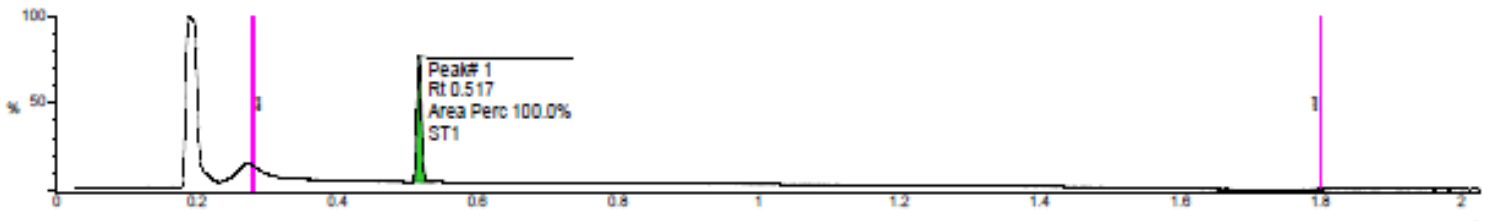

edd-6 302.1981 (301.7981-302 5681) w-0.8000

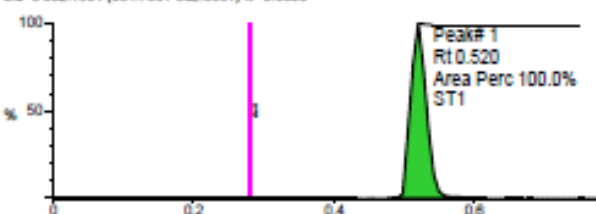

oid 11 11 300.1824 (2007 7824-500 5850) w-0.8000

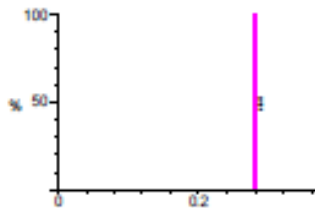

LLN Nr: ELN098-0663-A01

Acquisition time: 5/28/2020 9:55 AM Result

GOOD

100.0000

302.3000

0.5170
UV214 [4.4EOS] T1-EICI+0N+HII [5:

Poak $190.620 \mathrm{~min} \mathbf{M} 3(+)$ Es

4.0607 onte

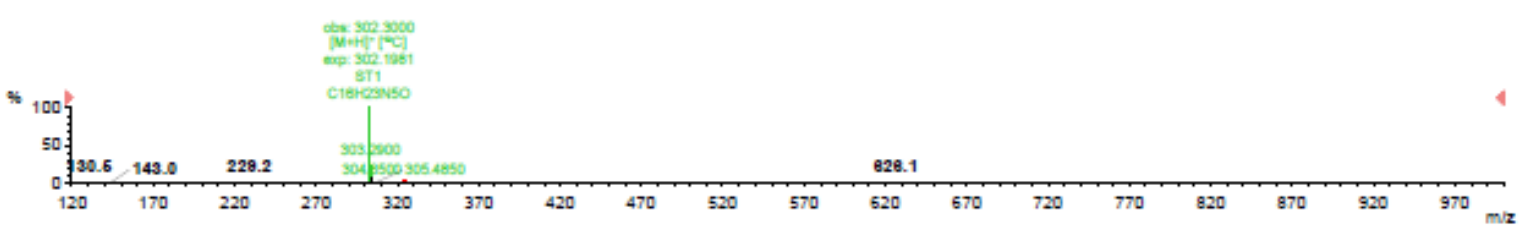


Compound 7c:

Sample Name: LCMS384-20200803-5_G23

\section{Expression}

Auto-Comments

Auto-Summary

Purity

Target Mass

Target RT

[V]212.216]

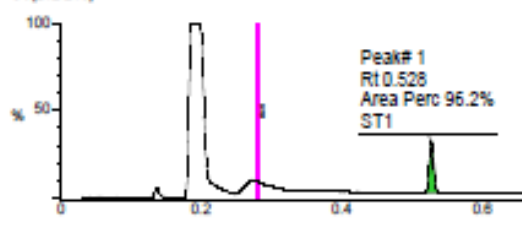

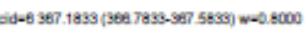

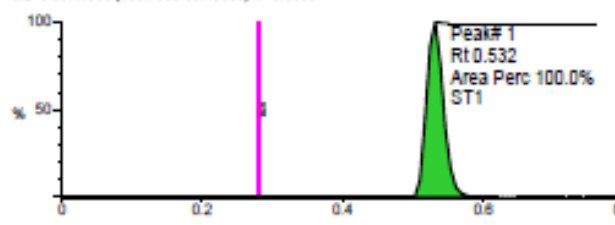

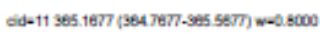

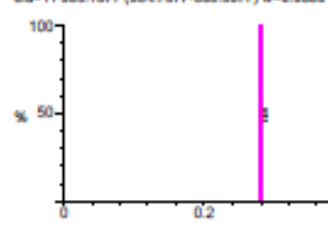

ACT No: ACT-707785

Instrument: ICHALW-DL00012-TQD
ELN Nr: ELN227-1507_AOB

Acquisition time: 8/3/2020 10:08 PM

Result

GOOD

96.2000

387.3000

0.5280

UV214[5,4EOS ]

Rit 0.925

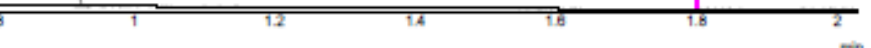

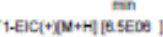

4.7E08 onte

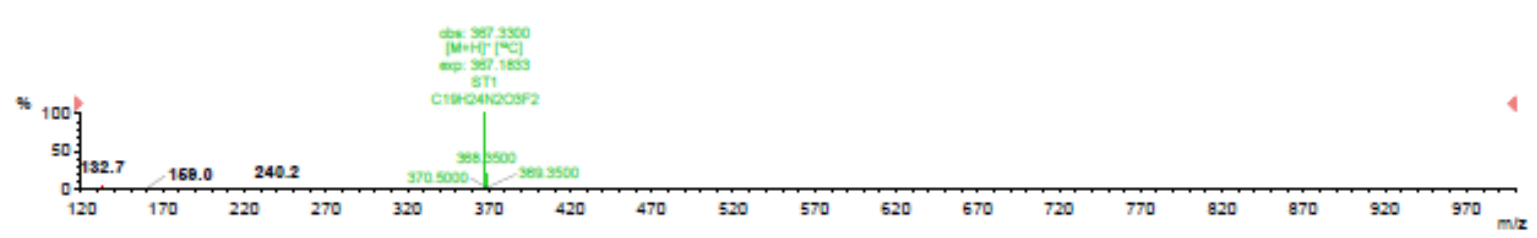


Compound 7d:

3: UV Detector: 212_216
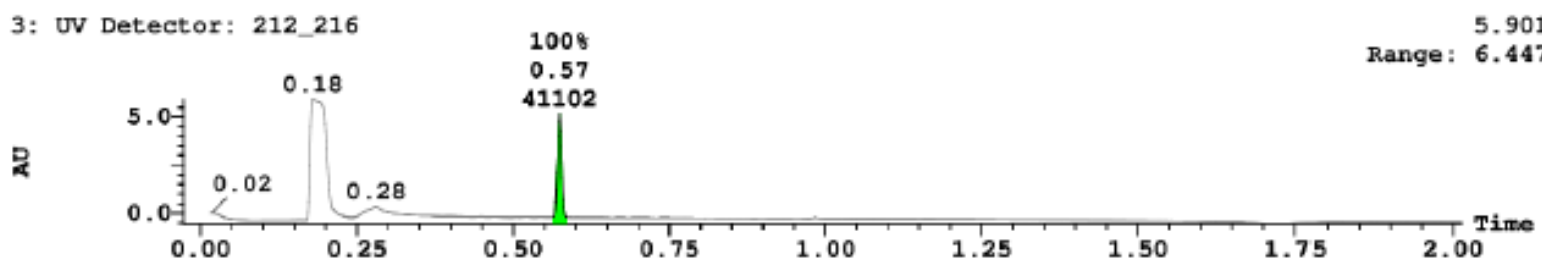

$\begin{array}{ll}1: \text { MS ESt : } 356.2091 .0000 \mathrm{Da} & 1008 \\ & 0.57\end{array}$

8. $7 e+007$

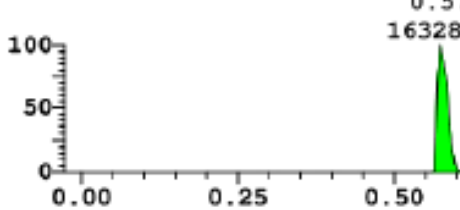

$$
2833
$$
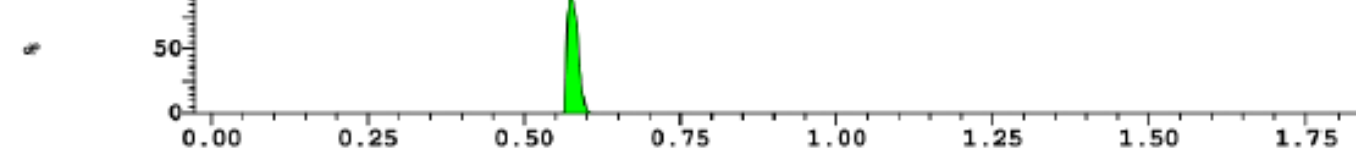

2. 00

2: MS ES- : $354.1931 .0000 \mathrm{Da}$

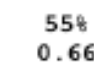

1. $6 e+00$
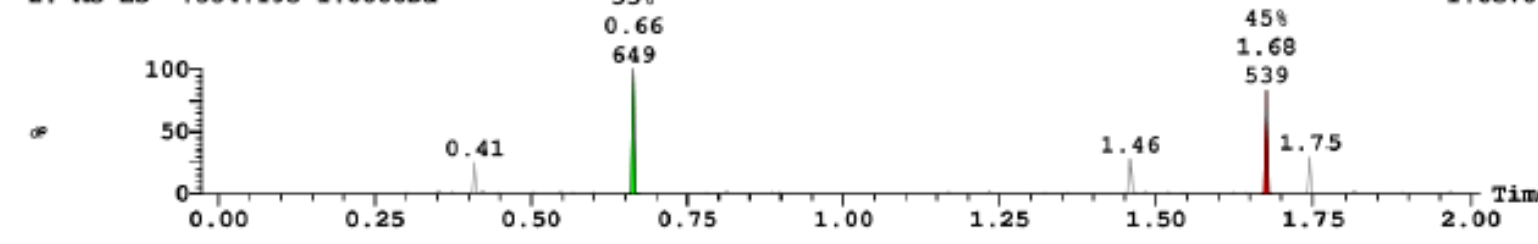

(1) ELSD Signal

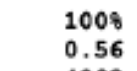

号

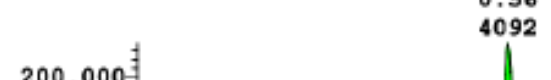

Range: 277.88

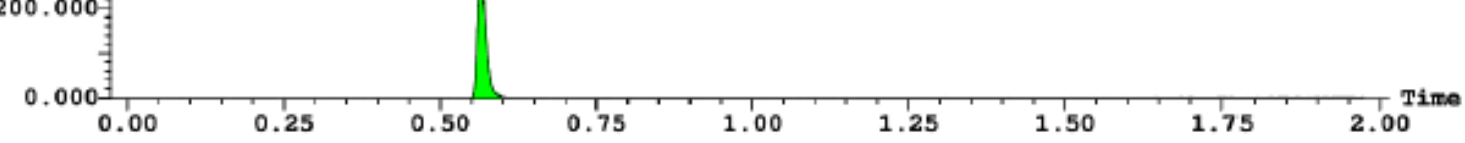

2: (Time: 0.57$)$ Combine (91:95-(70:73+118:121))

1 :NS ES+

356.3

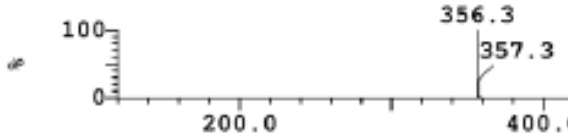

600.0

800.0

1000.0 
Compound 7e:
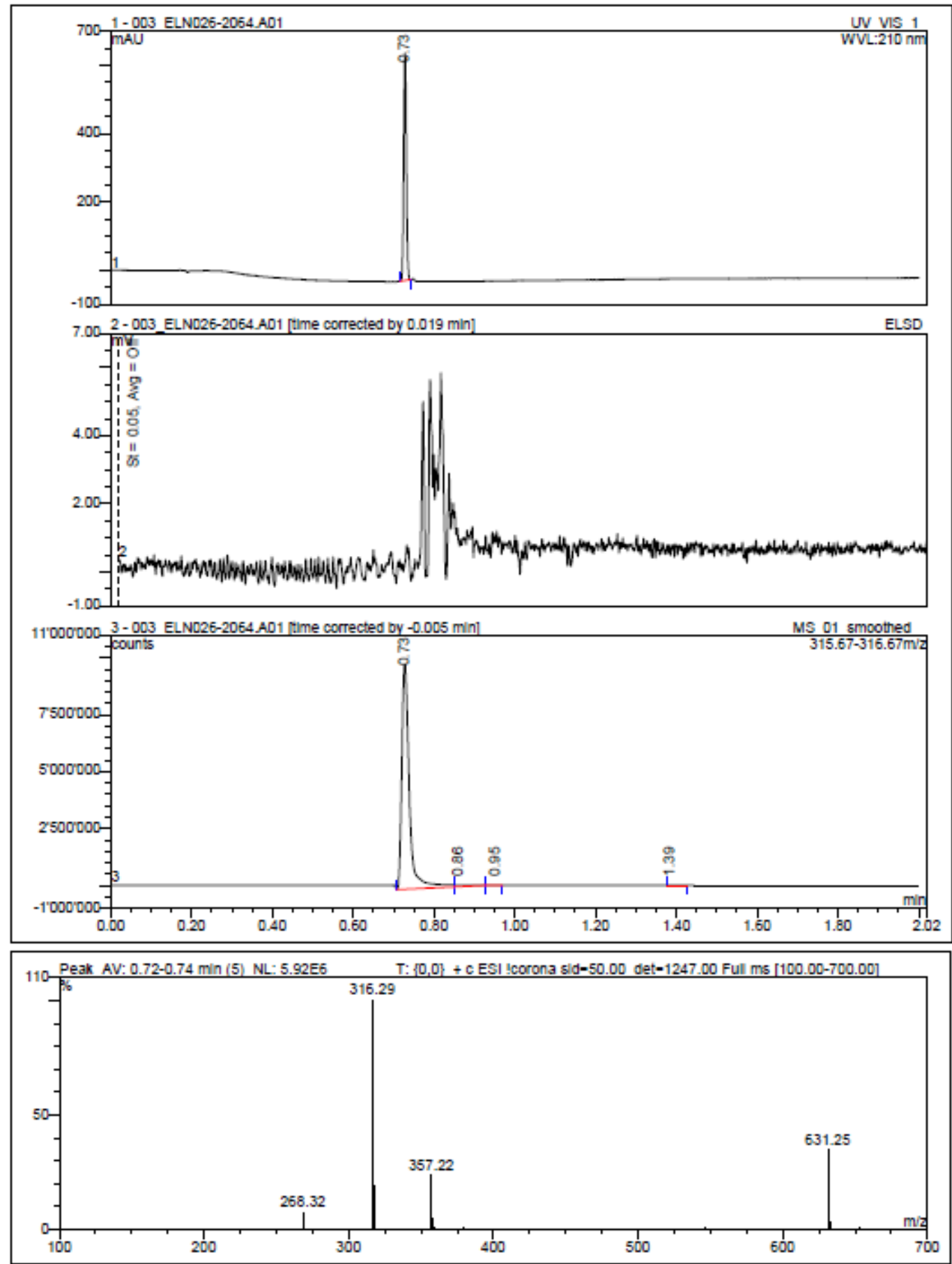
Compound 7f:

Sample Name: LCMSP384-20200528-1_003 ACT No: ACT-707100

Instrument: ICHALW-DL00021-SQD

Expression

Auto-Comments

Auto-Summary

Purity

Target Mass

Target RT

U. [212-216]

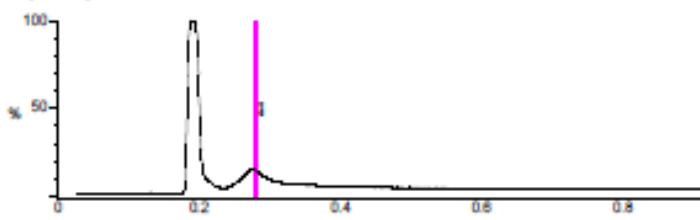

Sd-6 316.1773 (315 7775-916.577s) w-0.8000

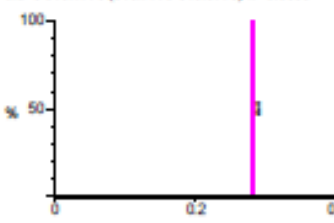

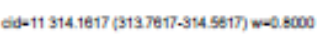

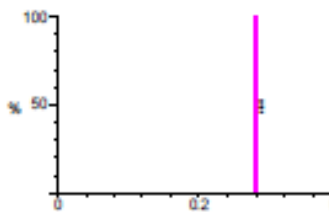

Posk 1 ggo.825 min Ma(+) Es

\% 10

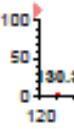

\section{Peala 1}

Rt 0.925

Area Perc 100.0\%

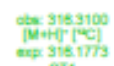

ELN Nr: ELN098-0663-A04

Acquisition time: 5/28/2020 9:15 AM Result

GOOD

100.0000

316.3000

0.9210

UV214[4.4EOE] ]

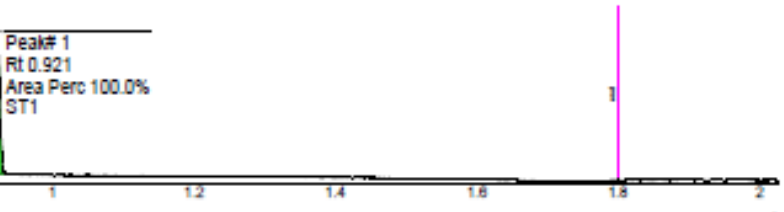

T1-EICI+10N+HI [37EO?

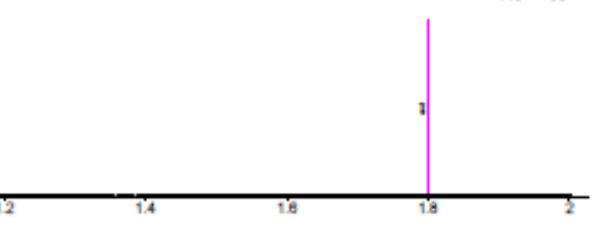

T1-EICF|MV-Tin [1.0 :

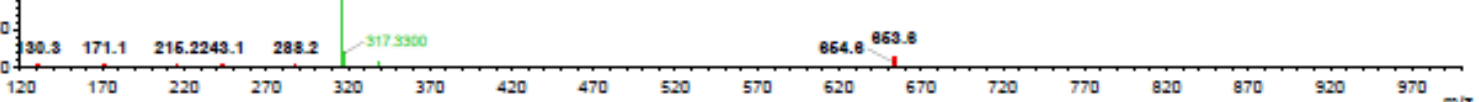


Compound 7g:

Sample Name: LCMSP384-20200528-1_K05 ACT No: ACT-707109

Instrument: ICHALW-DL00021-SQD

\section{Expression}

Auto-Comments

Auto-Summary

Purity

Target Mass

Target RT

บข:212.216]

${ }^{100} 7$.

$\times 50-$

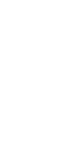

Area Perc $100.0 \%$

Area Per
ST1

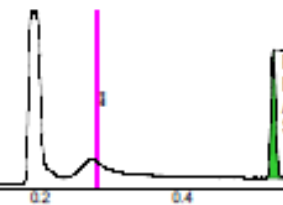

ELN Nr: ELN098-0683.B02

Acquisition time: 5/28/2020 9:32 AM

Result

GOOD

100.0000

314.3000

0.5280

UV214 [4.4EOE]

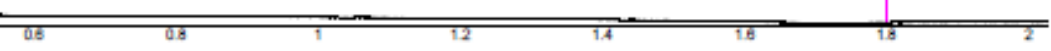

Sid-6 314.1951 (3197961-314. 5061) w-0:8000

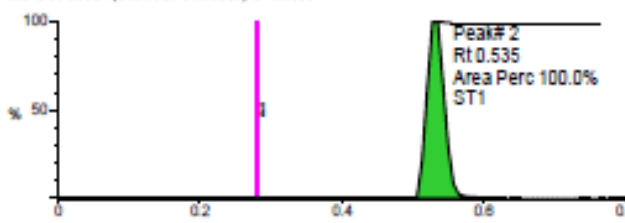

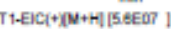

id-11 312.1824 (311.7624-312 5825) wa $w .9800$
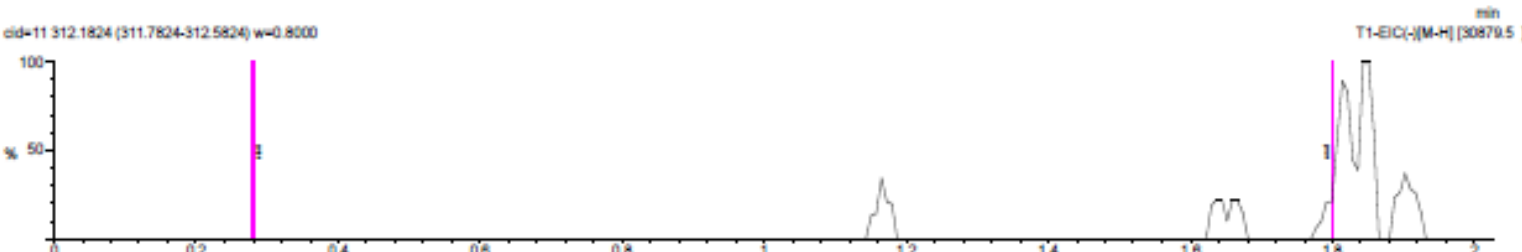

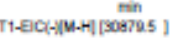

Poak 1 go.313 min Ms(+) Es

$1 E 06$ onte

100148.8

${ }^{100}$ ]30.2 148.8

21.6 30.159.8 198.1237 .0

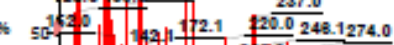

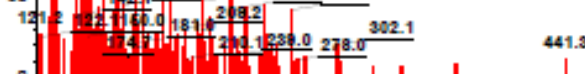

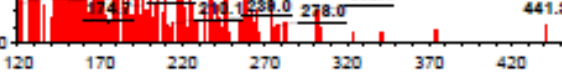

Poak 2 ge. $635 \mathrm{~min} \mathbf{M} 3(+)$ Es

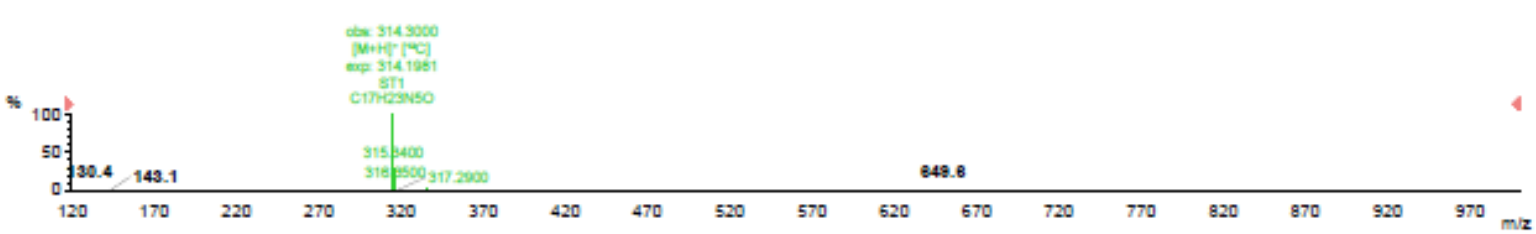


Compound 7h:

Sample Name: LCMS384-20200715-2_C01

ACT No: ACT-709860

Instrument: ICHALW-DL00012-TQD

\section{Expression}

Auto-Comments

Auto-Summary

Purity

Target Mass

Target RT

UV:212216]

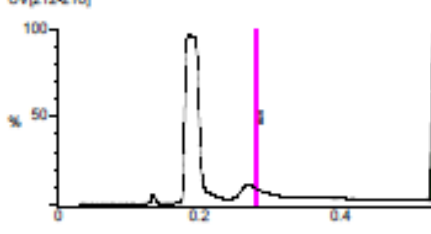

4-6 316.2197 (315.819-318. 8197$) *-0.8000$

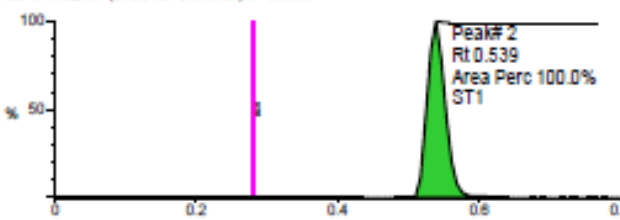

od-11 314.1981 (3197981-314. 51861) w-0.5000
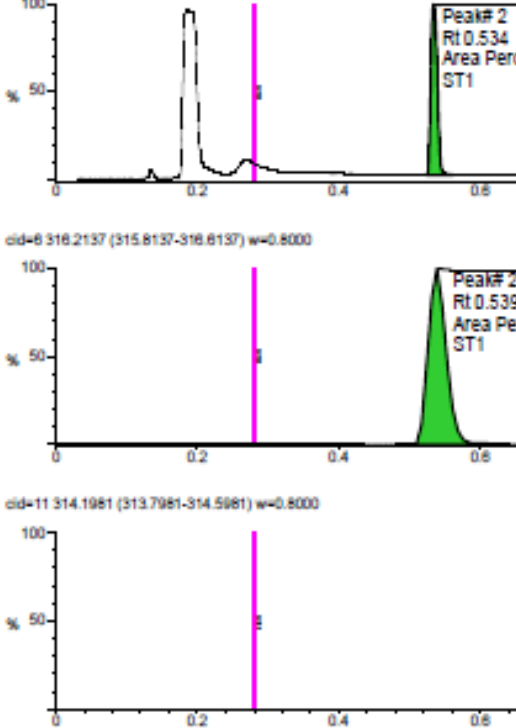

Ri 0.534

Are
ELN Nr: ELNO26-2083_A04

Acquisition time: 7/15/2020 12:14 PM Result

GOOD

99.3000

316.4000

0.5340

UV214 [5.SEDS ]

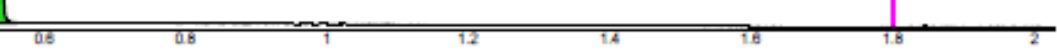

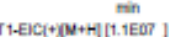

Poak 1 ge .398 min $M a(+)$ Es

1.1E06 onte

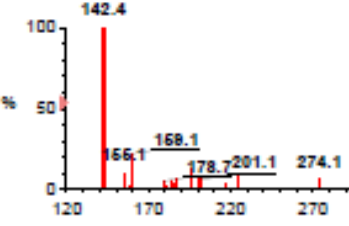

Poak 2g0.639 min M3i+) Ea

3.6508 onts

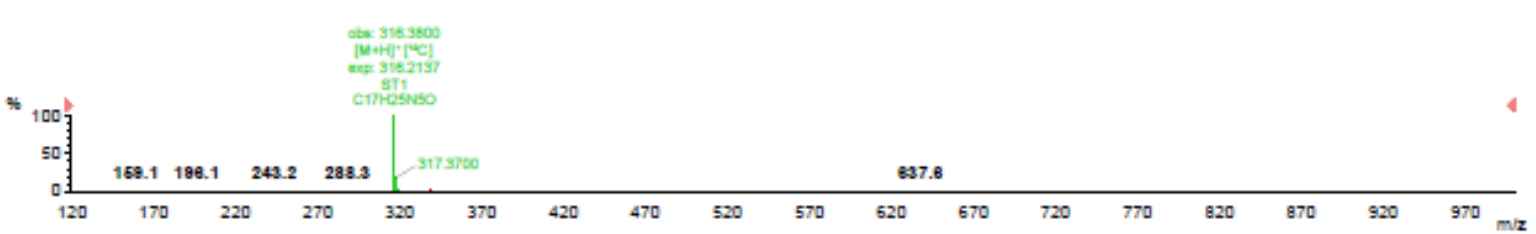


Compound 7i:

3: UV Detector: 212_216

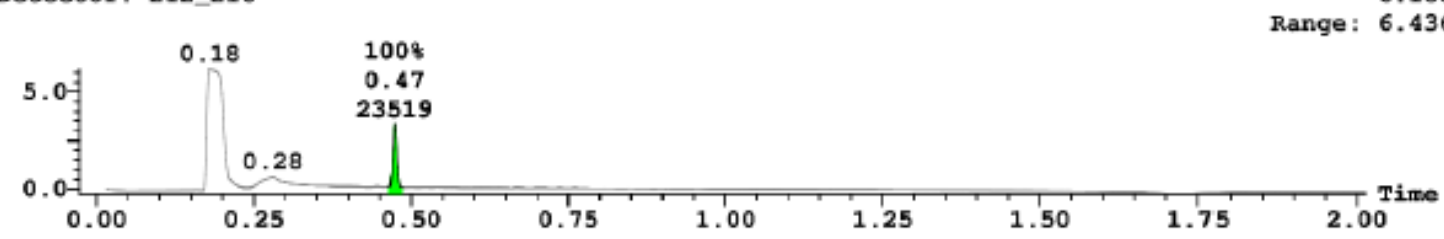

1: MS ESt : $353.2041 .0000 \mathrm{Da} \quad 100$ z

7. 8 e+ +007

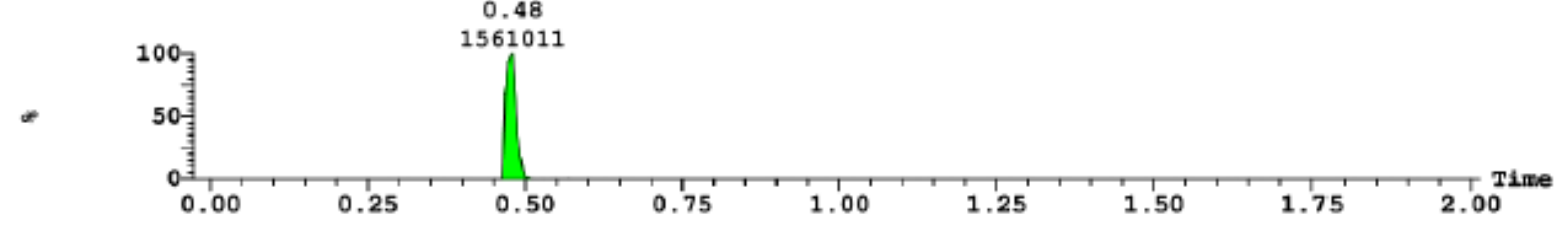

2: MS ES- :351.188 1.0000Da

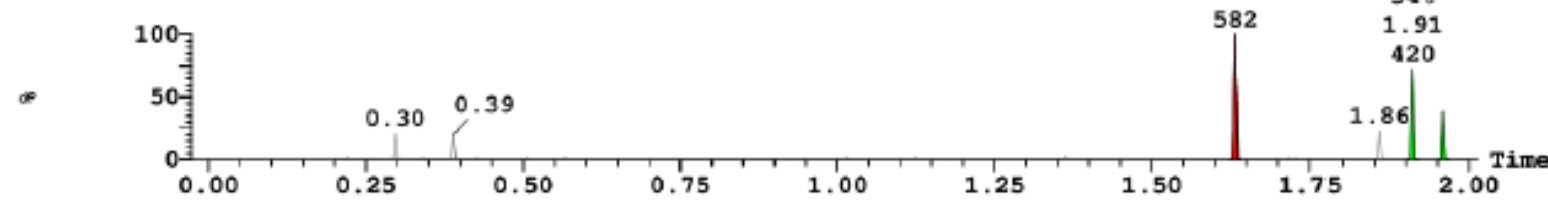

(1) ELSD Signal 1009

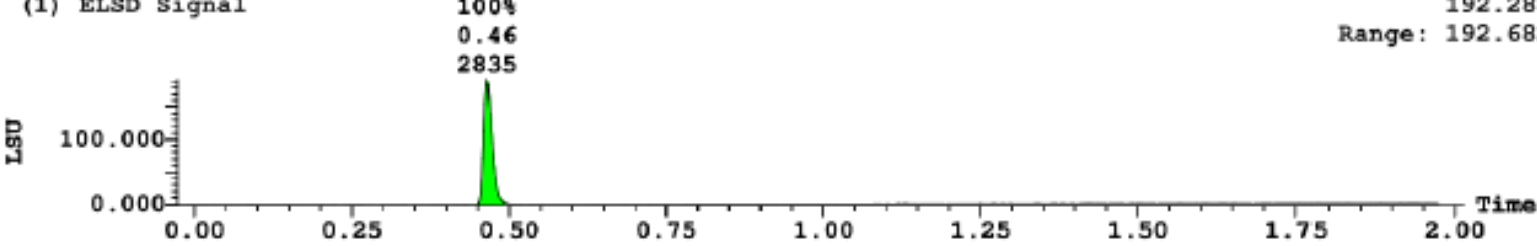

2: (Time: 0.47$)$ Combine (66:71-(46:49+89:91))

1:MS ES+

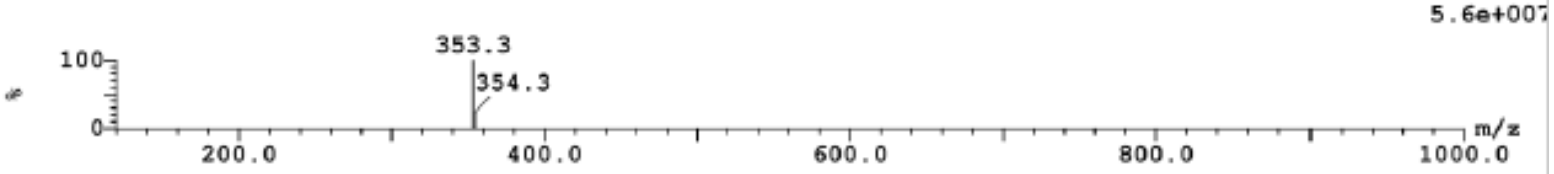


Compound $7 \mathbf{j}$ :

Sample Name: LCMS384-20200803-5_A23

\section{Expression}

Auto-Comments

Auto-Summary

Purity

Target Mass

Target RT

uพ[212216]

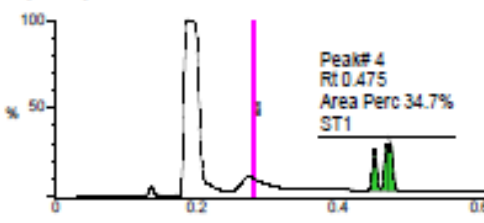

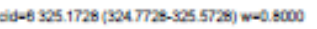

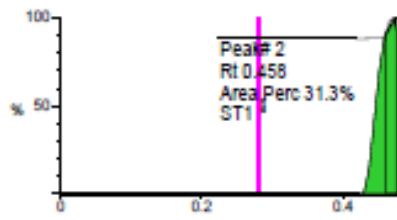

ACT No: ACT-709678

Instrument: ICHALW-DL00012-TQD

sid-11 \$22.1571 (322.7571-529.5571) w-0.8000

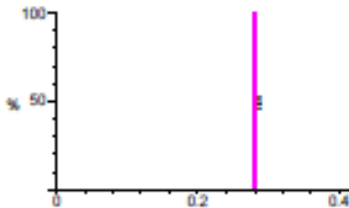

ELN Nr: ELN227-1507-A02

Acquisition time: 8/3/2020 9:59 PM

Result

Possible Isomers

GOOD

98.2000

325.3000

0.4750

UV214 [5.SEDE ]

Poak 2g0.458 min us?(+) Es

4.3E0B onte
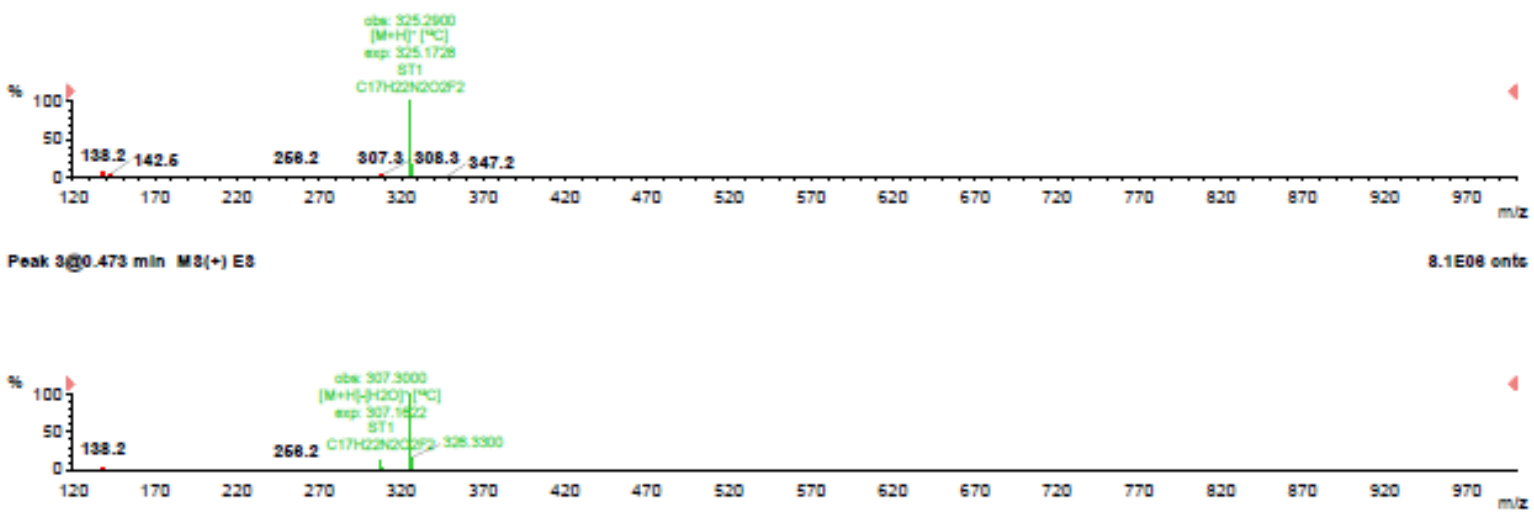
Compound 7k:

Sample Name: LCMS384-20200512-1_105

Expression

Auto-Comments

Auto-Summary

Purity

Target Mass

Target RT

uv[212216]

Peakt 1

Area Perc 100.0\%

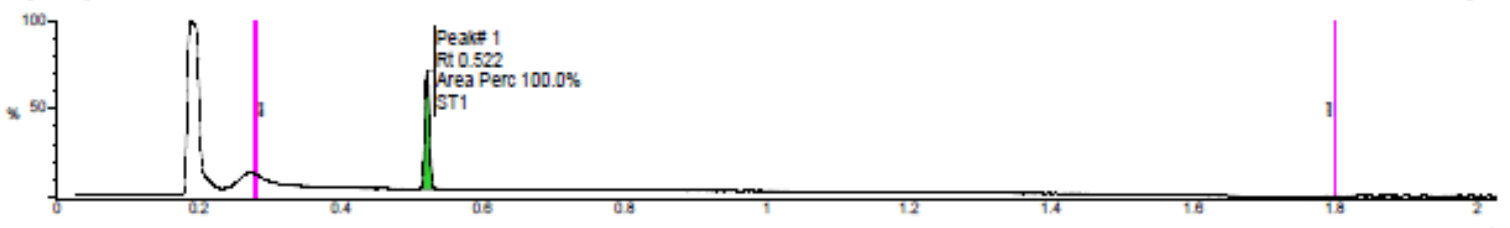

ACT No: ACT-709631

Instrument: ICHALW-DL00021-SQD

LN Nr: ELNO26-2066_A01

Acquisition time: 5/12/2020 4:51 PM Result

GOOD

100.0000

314.3000

0.5220

UV214 [4EEOS ]

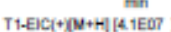
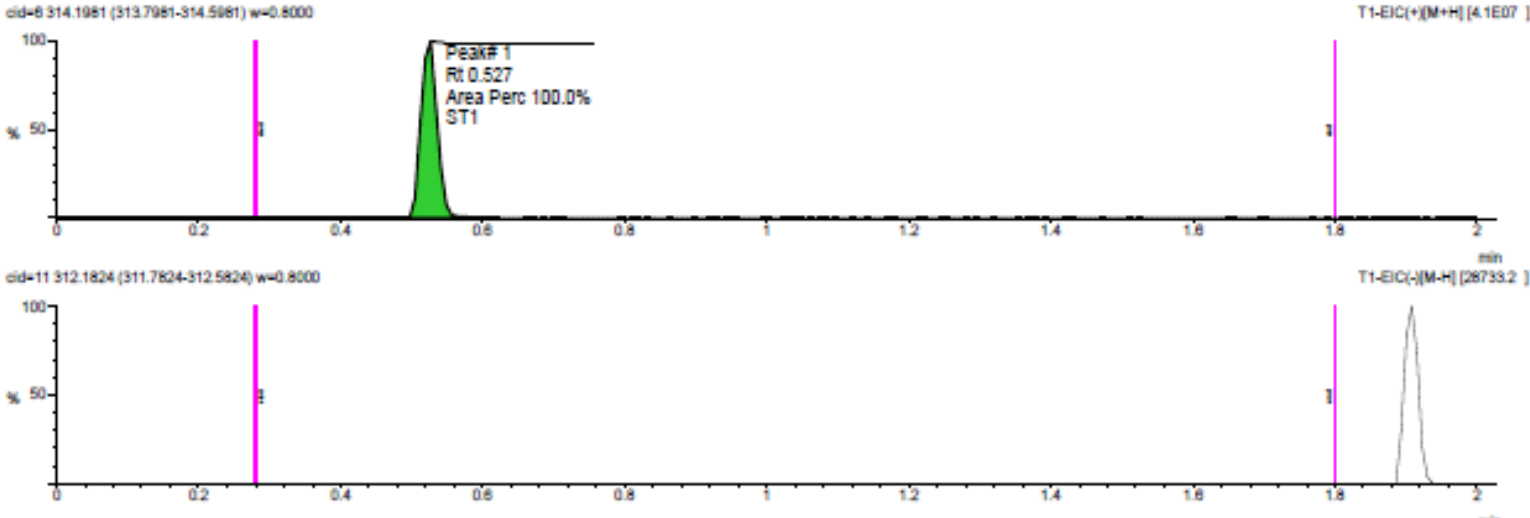

Poak 190.627 min $\mathbf{m} 3(+)$ Es

2.8E07 onte

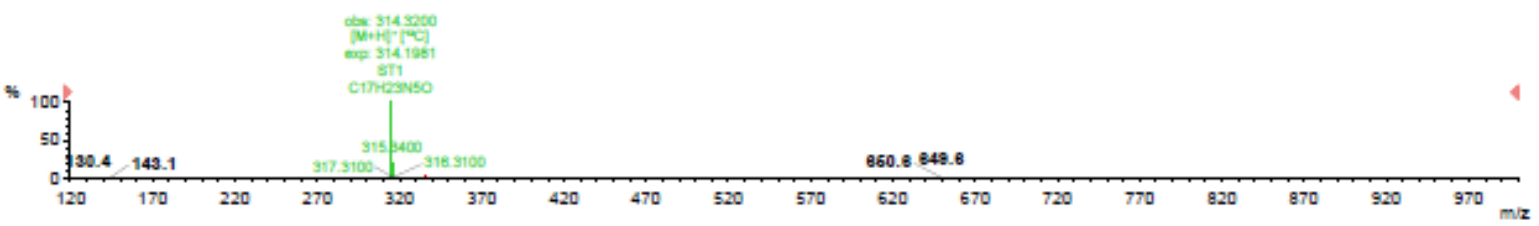


Compound 71:

3: UV Detector: 212_216

0.18

968

14115

是

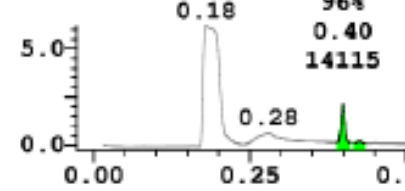

1: MS ESt : $311.1571 .0000 \mathrm{Da}$

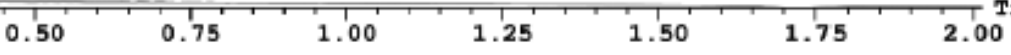

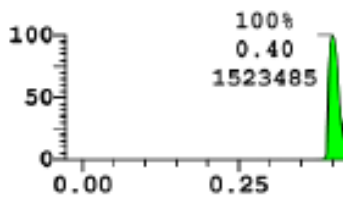

2: US ES- :309.141 1.0000Da
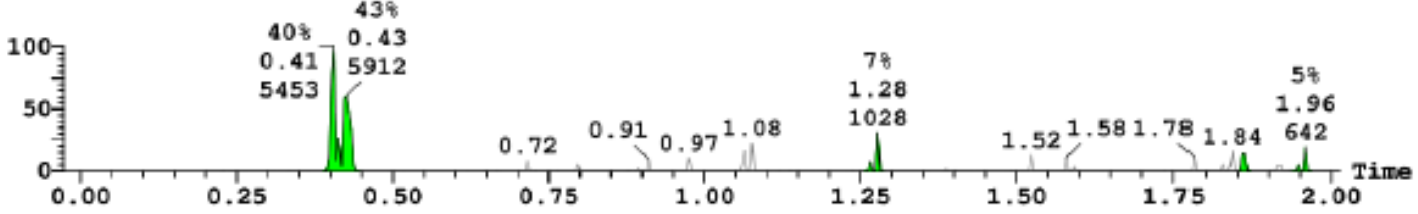

(1) ELSD Signal
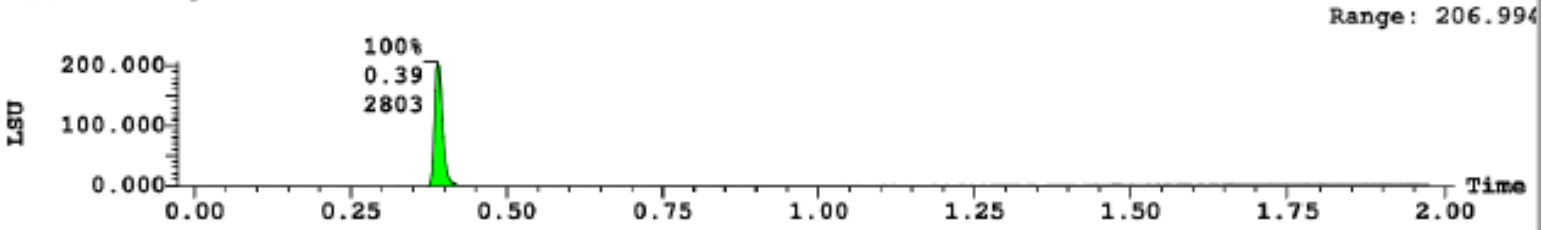

2: (Time: 0.40$)$ Combine $(47: 52-(28: 30+70: 73))$

1 :NS ES+

4. $8 e+00$

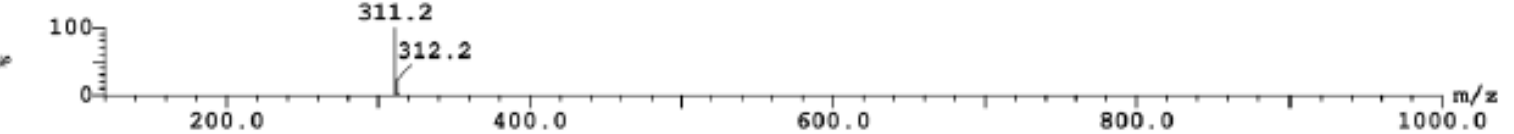

3: (Time: 0.40$)$ Combine $(48: 52-(26: 29+78: 81))$

1 :NS ES+

311.2

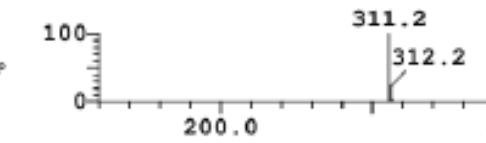

400.0 
Compound 7m:

3: UV Detector: 212_216

足

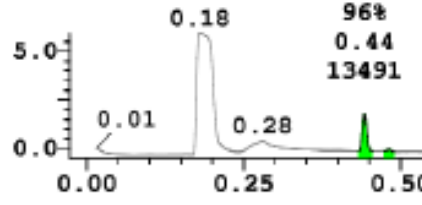

3491

1: MS ES+ : 339.188 1.0000Da

8. 0 e+ +007
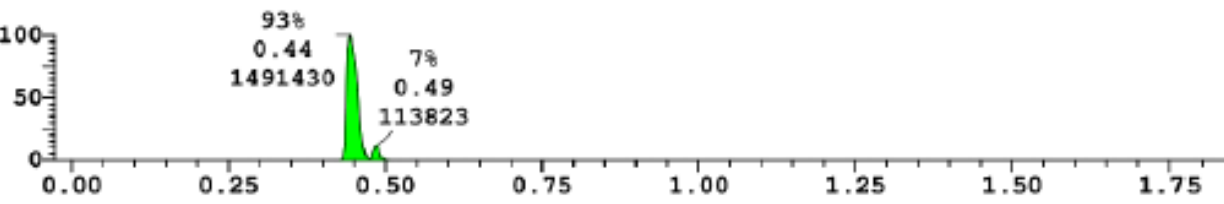

2.00

2: MS ES- : $337.1731 .0000 \mathrm{Da} \quad 49 \%$

8. $7 e+005$

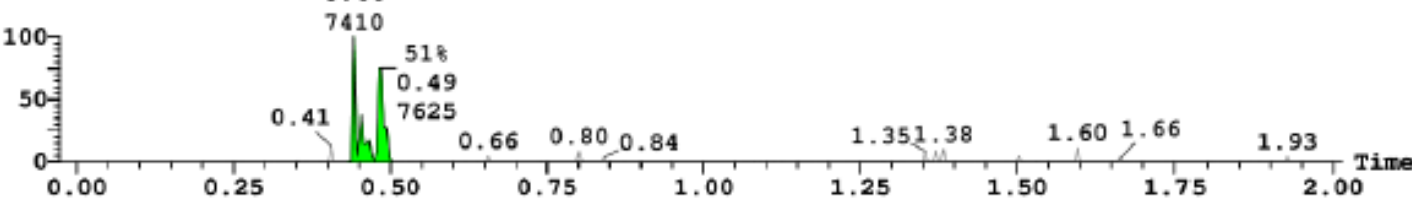

(1) ELSD Signal

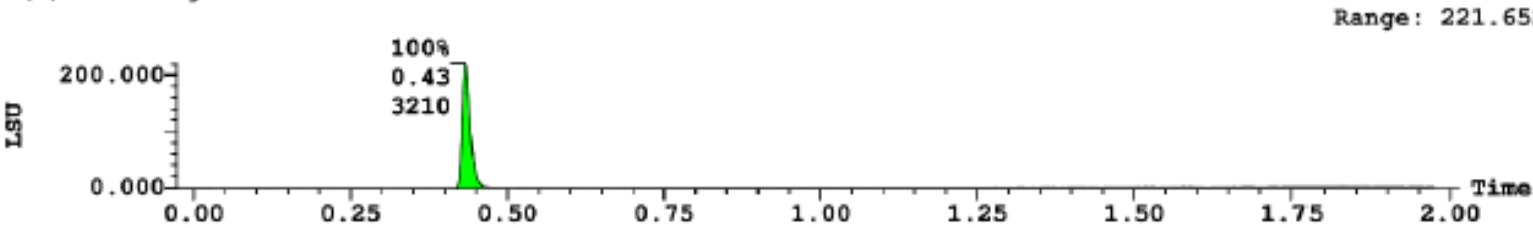

3: (Time: 0.44$)$ Combine $(58: 63-(39: 41+81: 84)$ )

1:NS ES+

339.3

$+\quad 100-$

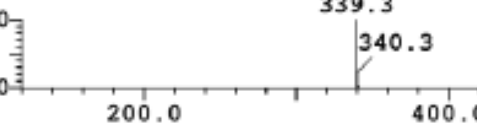

00.0

600.0

800.0

1000.0

4: (Time: 0.44$)$ Combine (59:63-(37:40+85:88))

1 :NS ES+

339.3

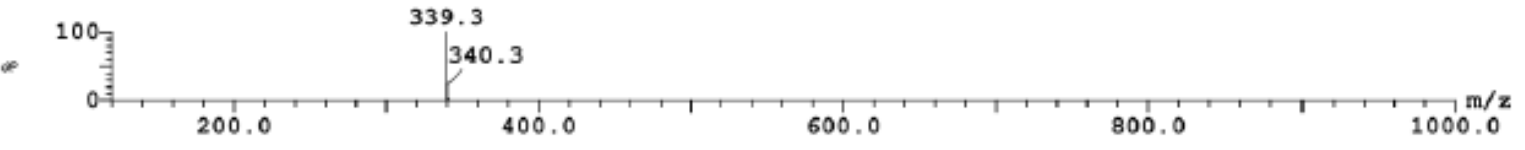


Compound 7n:

3: UV Detector: 212 216

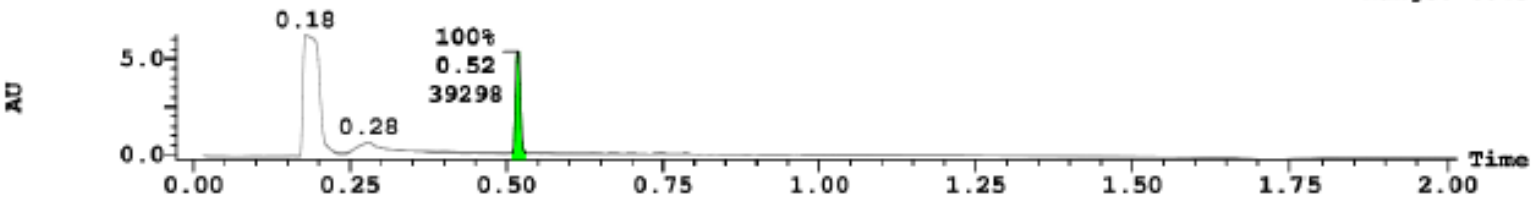

1: MS ESt : $328.2141 .0000 \mathrm{Da} \quad 100 \%$

7. $7 e+00$
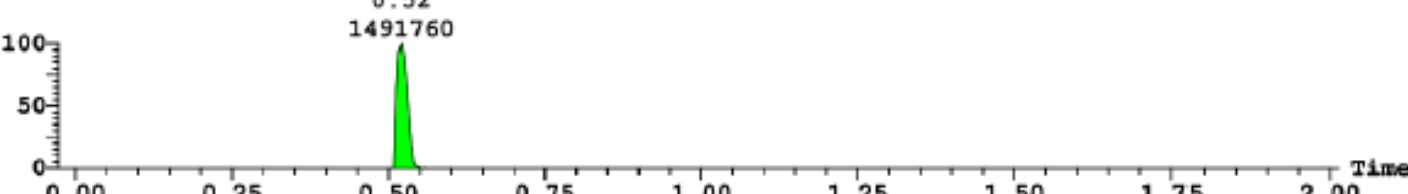

2: MS ES- :326.198 1.0000Da

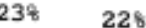

$1.2 e+005$ $1.11 \quad 1.28 \quad 18 \%$

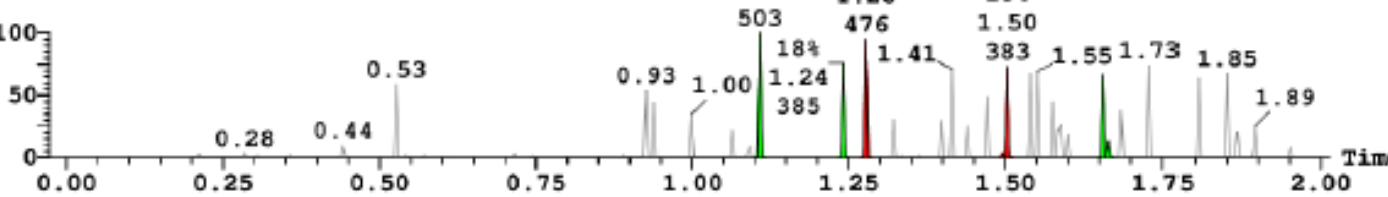

(1) ELSD Signal

\section{8}

$195.80 \mathrm{C}$

0.51
2750

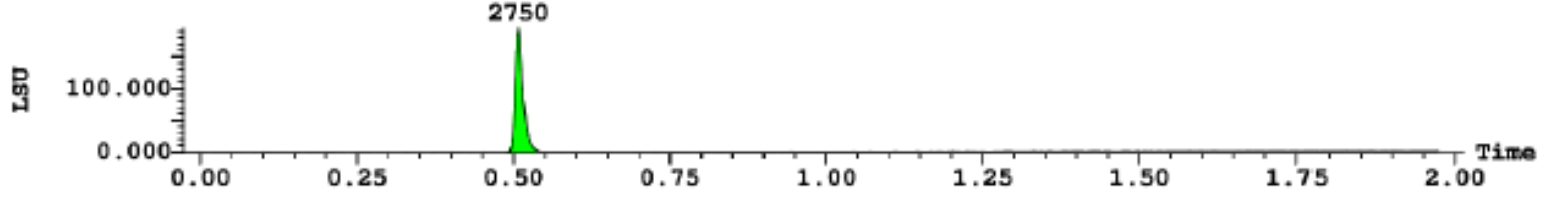

2: (Time: 0.52$)$ Combine $(77: 82-(57: 60+100: 102)$ )
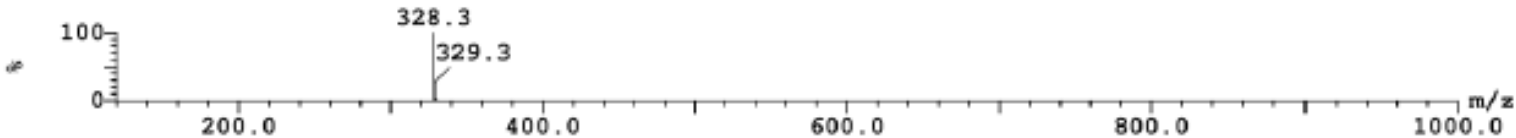

3: (Time: 0.52$)$ Combine (78:82-(57:60+104:107))

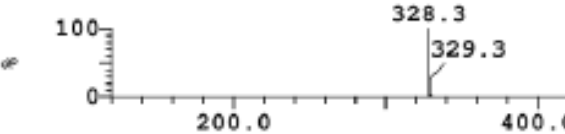


Compound 7o:

Sample Name: LCMSP384-20200528-1_O09 ACT No: ACT-709853

Instrument:

ELN Nr: ELNO26-1143_A01

\section{Expression}

Acquisition time: 5/28/2020 10:24 AM

Result

Auto-Comments

Auto-Summary

GOOD

Purity

100.0000

Target Mass

379.4000

Target RT

0.5530

uv[212216]

UV214 [4.4EDE]

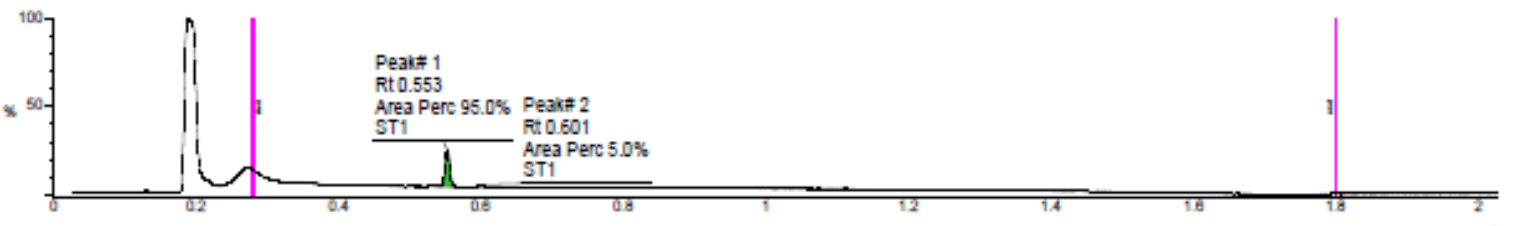

$\left.\begin{array}{c}\min \\ \mathrm{T} 1-\mathrm{EICl}+[\mathrm{N}+\mathrm{H}][52 \mathrm{EO}]\end{array}\right]$

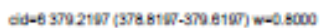

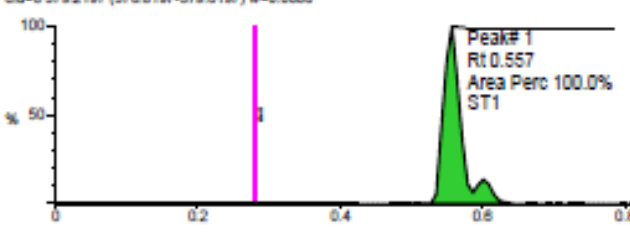

Gid-11 377 2041 (9726 6041-377. .0041) w-0.8000

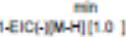
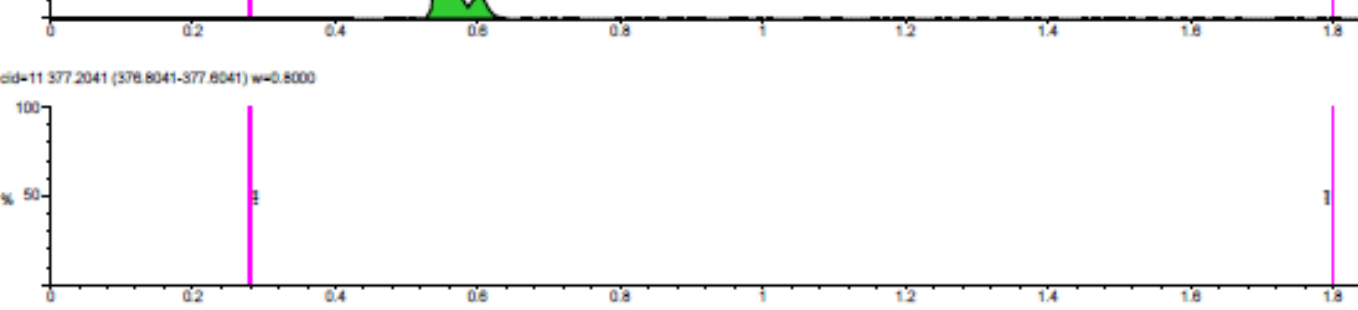

Poak 190.657 min $\mathbf{M} 3(+)$ Es

$3.6 E 07$ onte

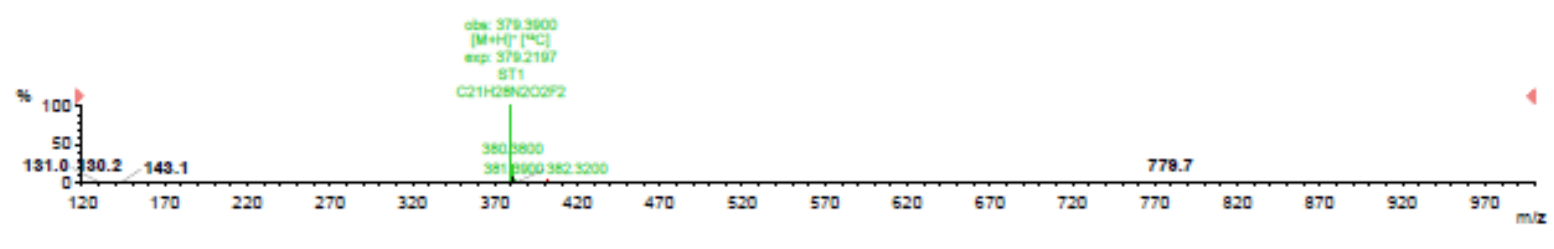

Poak 2g0.601 min $\mathbf{M} 3(+)$ Es

6.4E08 onte

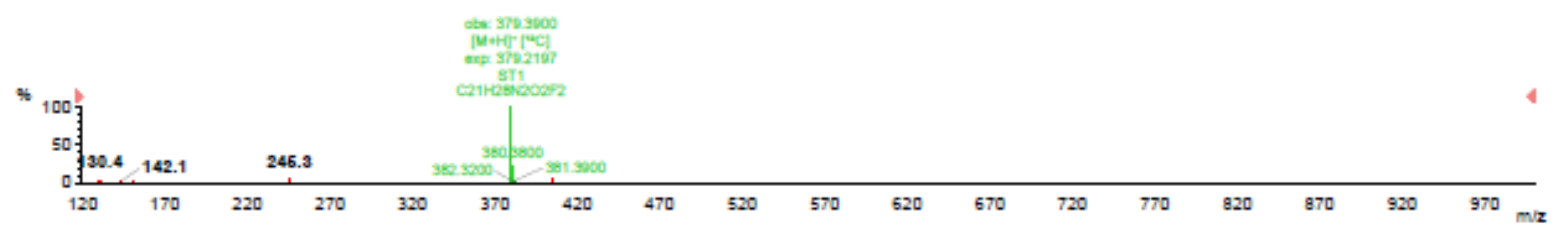


Compound 7p:

Sample Name: LCMS384-20200803-5_M23 ACT No: ACT-709865

Instrument: ICHALW-DL00012-TQD

Expression

Auto-Comments

Auto-Summary

Purity

Target Mass

Target RT

Uง:212216]

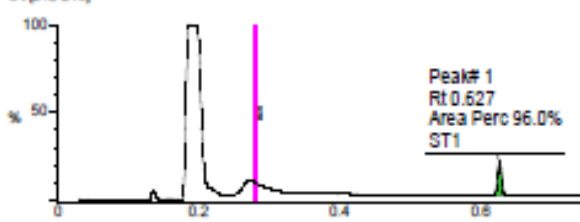

ELN Nr: ELN227-1507-A04

Acquisition time: 8/3/2020 10:17 PM Result

GOOD

96.0000

368.4000

0.6270

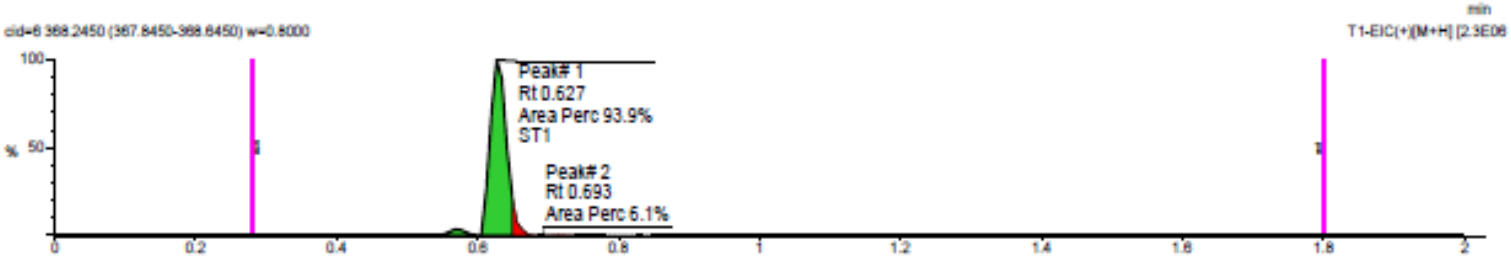

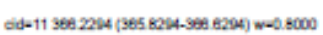

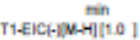
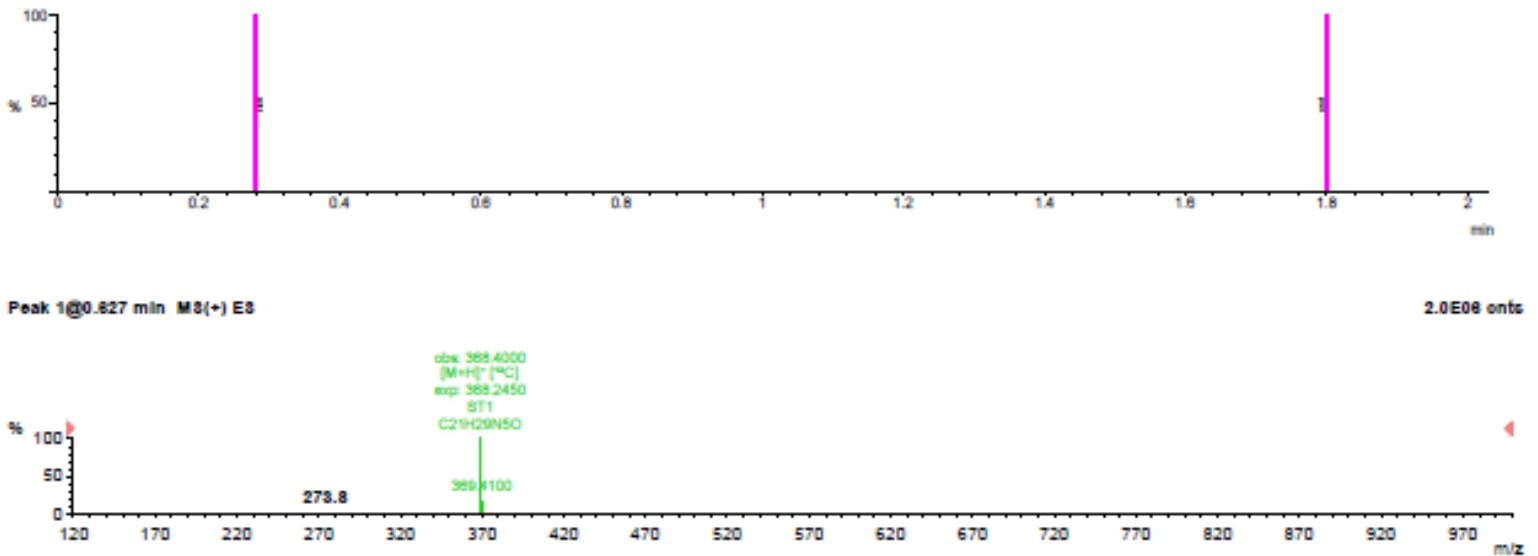
Compound 8a:

3: UV Detector: 212_216

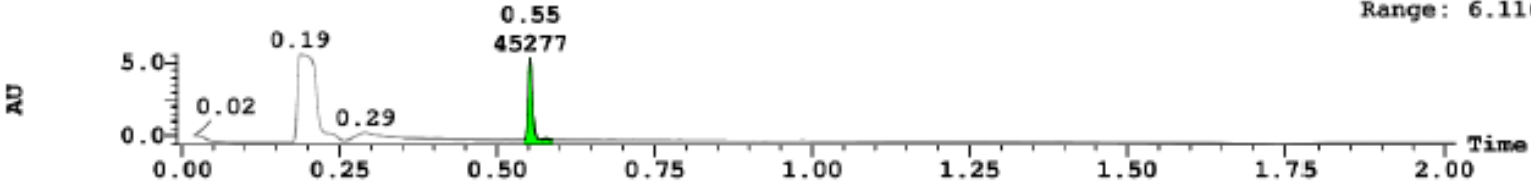

1 : MS ES+ : $354.2291 .0000 \mathrm{Da} \quad 1008$

9.2e+00:

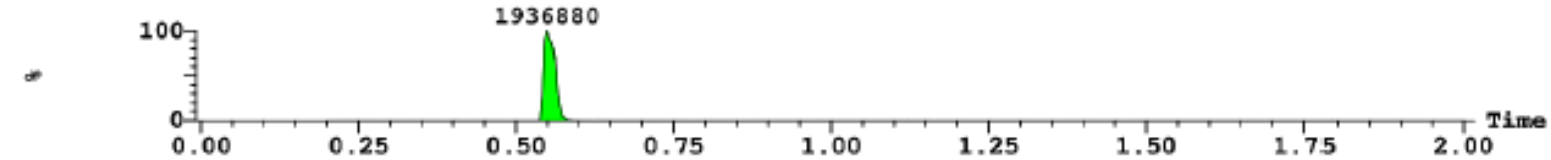

2: MS ES- :352.214 1.0000Da

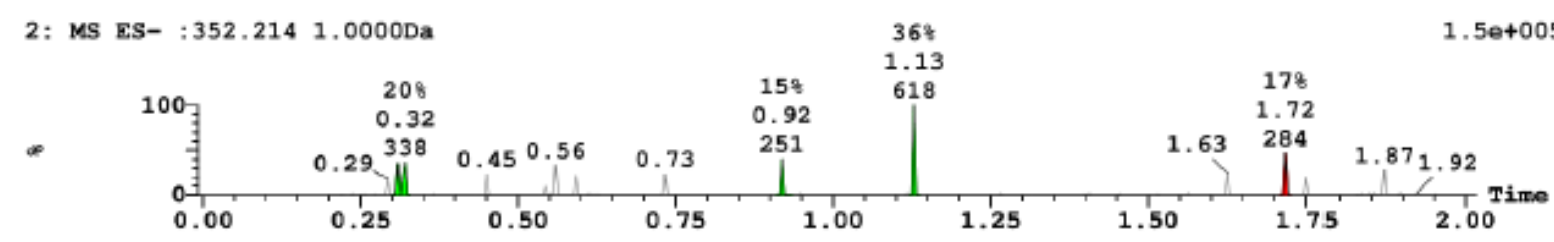

$\begin{array}{lll}\text { (1) ELSD Signal } & 1008 & 271.931 \\ & 0.56 & \text { Range: } 273.124\end{array}$

36 \%

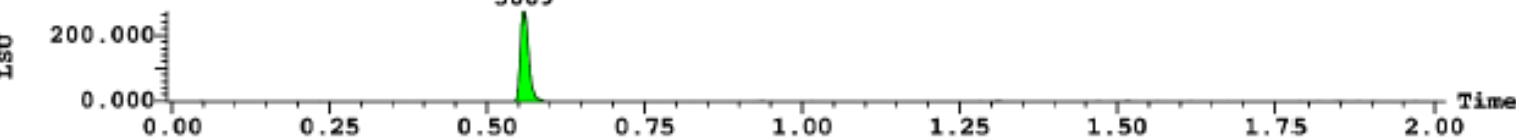

3: (Time: 0.55$)$ Combine $(85: 89-(64: 67+113: 116))$

1:NS ES+

5. 4e+00:

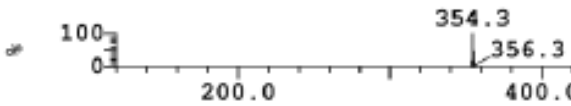


Compound 8b:

Sample Name: LCMS384-20200713-3_K01

Expression

Auto-Comments

Auto-Summary

Purity

Target Mass

Target RT

UVI212216]

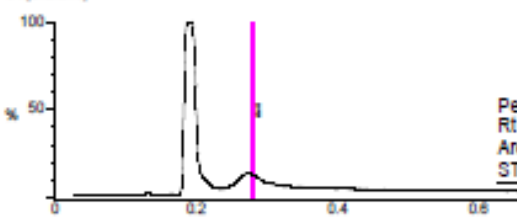

ACT No: ACT-710148

Instrument: ICHALW-DLO0021-SQD

Avea Perc $1000 \%$

id -6.432 .1287 (431.7287-432 5287) w-0.5000

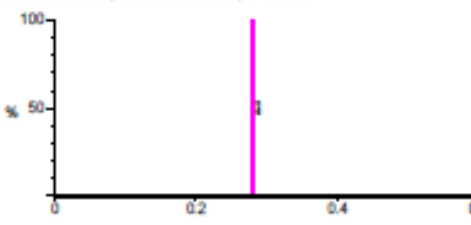

aid-11 490.1190 (4207190-490 5190) w-0.8000

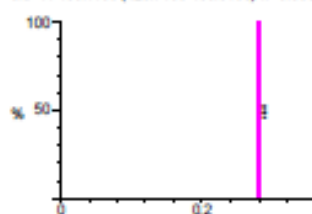

12

Area Perc $100.0 \%$

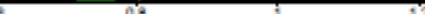

Poak 1 go.734 min $\mathbf{m} 8(+)$ Es
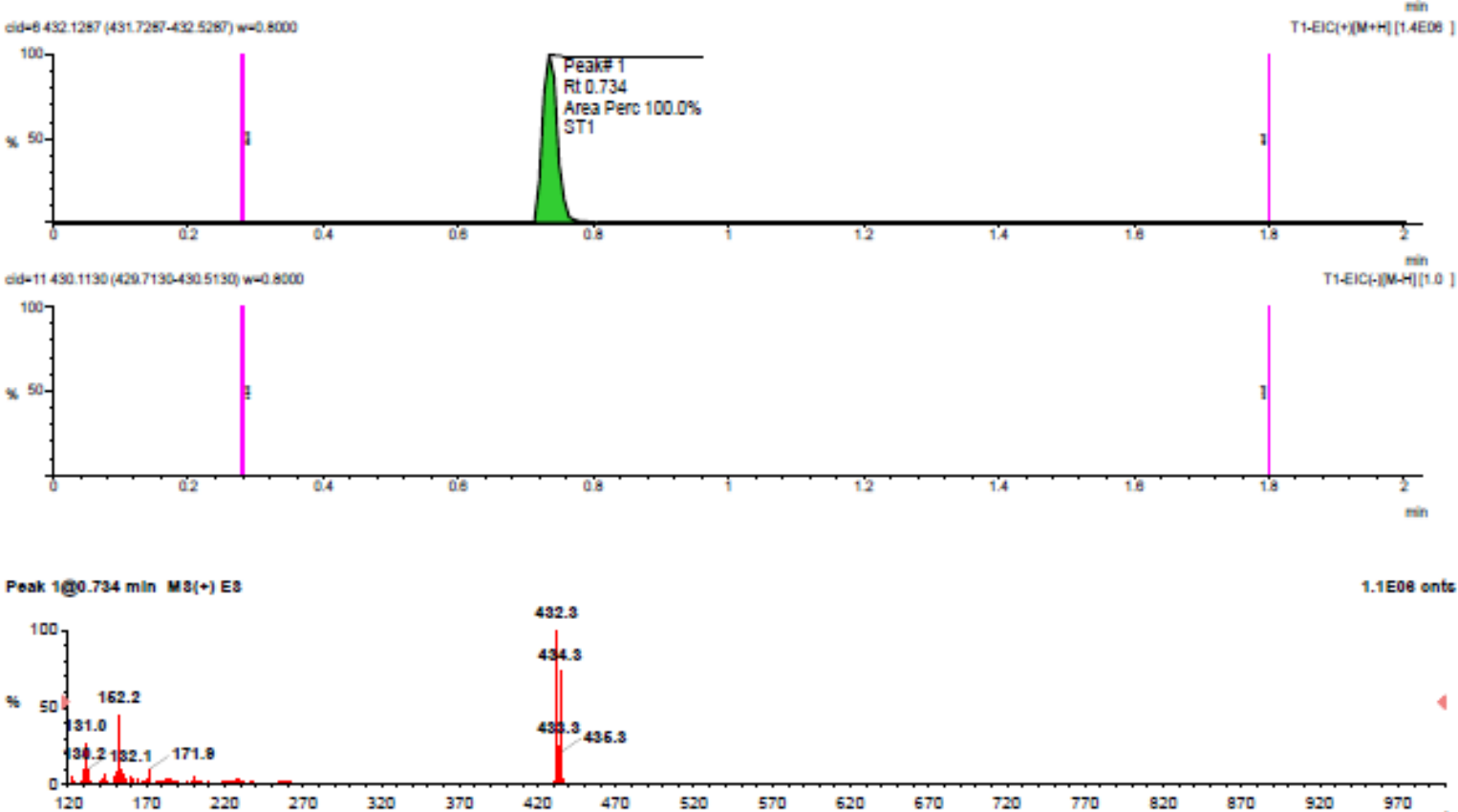

432.3

1.1 E0 onte
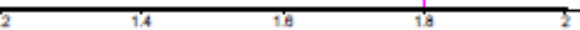

T1-EICF|M-Min

Acquisition time: 7/13/2020 3:31 PM

Result

GOOD

100.0000

0.0000

0.7360

UV214 [4.7EOS]

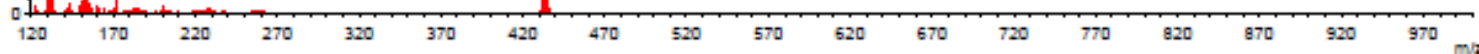


Compound 8c:

3: UV Detector: 212_216 Smooth (SG, 1×3)

号

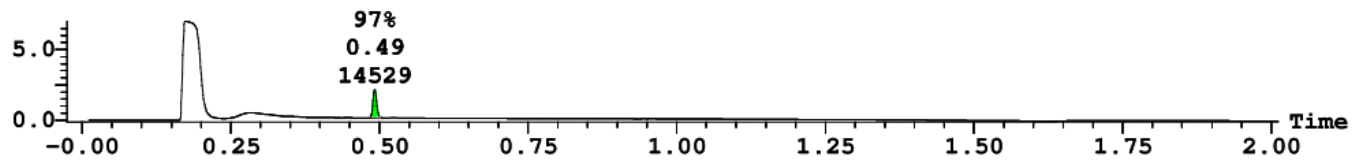

1: MS2 ESt : $365.231+343.2391 .0000 \mathrm{Da}$ Smooth (SG, $2 \times 1$ )

6. $3 e+007$

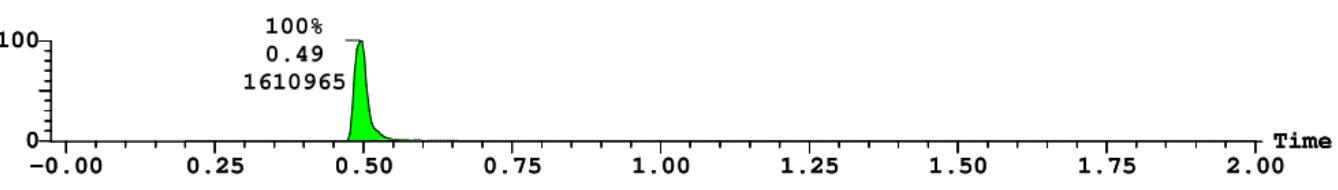

2: MS2 ES- : $387.231+341.2231 .0000 \mathrm{Da}$ Smooth (SG, $2 \times 1$ )

$1.7 e+004$

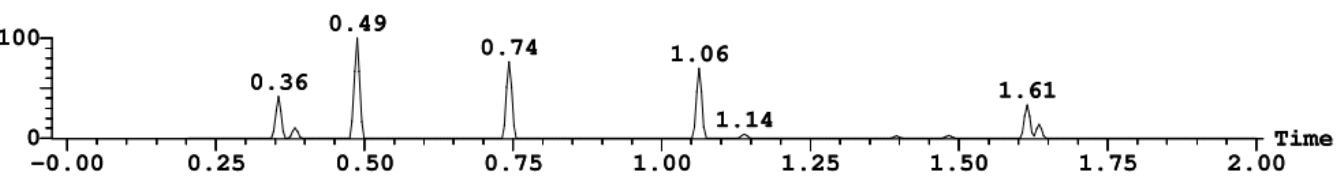

(2) ELSD Signal

$100 \%$

122.646

1008
0.49

Range: 122.713

照

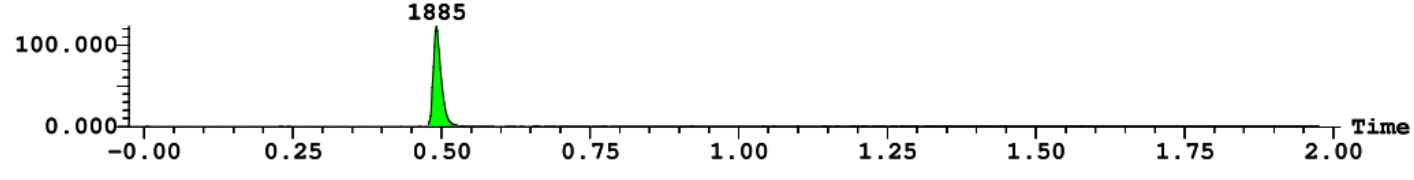

1:(Time: 0.49$)$ Combine $(71: 76-(54: 56+96: 99))$

$1:$ MS2 ES+

$5.3 e+007$

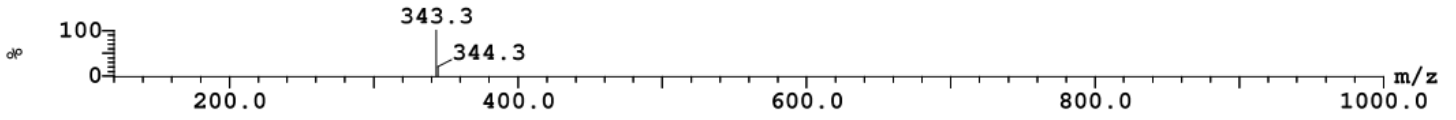


Compound 8d:

3: UV Detector: 212_216 Smooth (SG, 1x3)

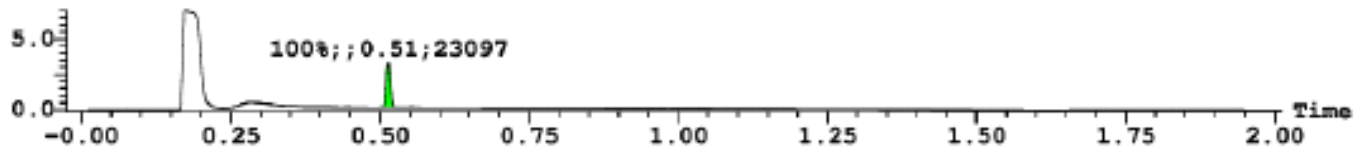

1: MS2 IS+ :383.221+361.229 1.0000Da Smooth (SG, 2x1)

7. $5 e+007$

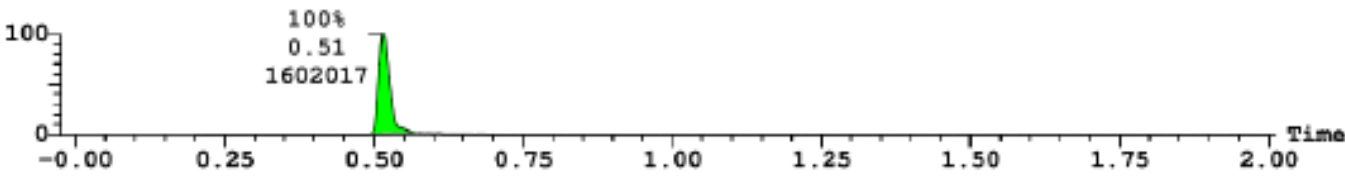

2: MS2 ES- :405,221+359.214 1.0000Da Smooth (SG, 2x1)

$4,6 e+004$

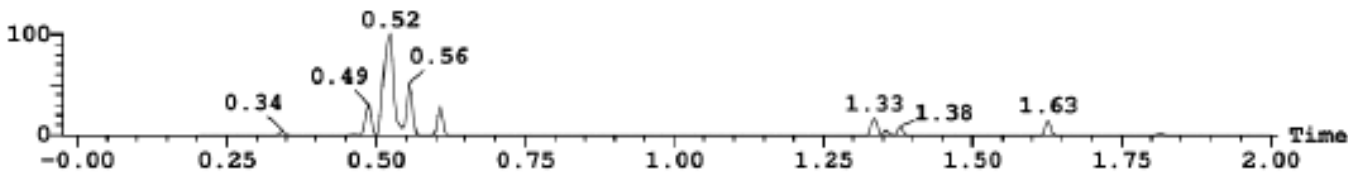

(2) ELSD Signal

117.106

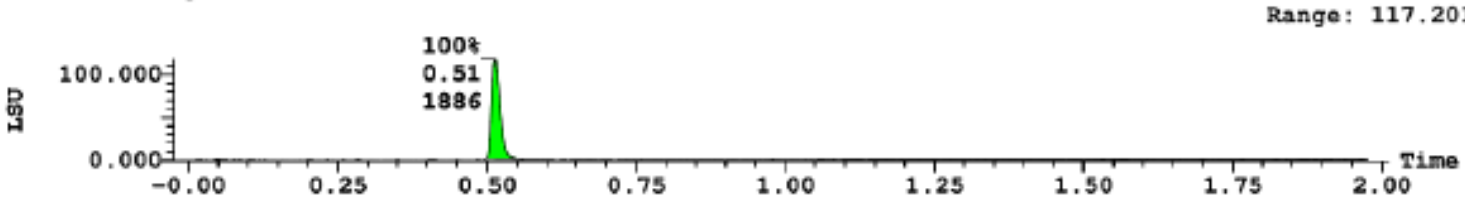

1: (Time: 0.51$)$ Combine $(76: 81-(60: 62+102: 105)$ )

1 :MS2 ES+

$={ }^{100}=0$ 
Compound 8e:

Sample Name: LCMS384-20200713-3_M01

\section{Expression}

Auto-Comments

Auto-Summary

Purity

Target Mass

Target RT

ACT No: ACT-730647

Instrument: ICHALW-DL00021-SQD

|งกาเ2-2)

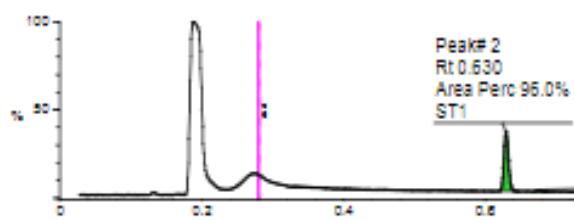

rea Perc $95.0 \%$

Peaks 3

Rt 0.676

Area Perc $2.3 \%$

ST1

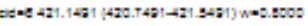

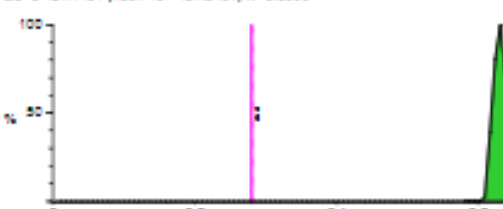

Peake 2

Ft 0.63

Area Perc 100.0\%

N Nr: ELN028-2064.A05

Acquisition time: $7 / 13 / 2020$ 3:34 PM

Result

GOOD

88.3000

423.3000

0.6300

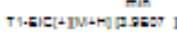

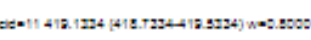

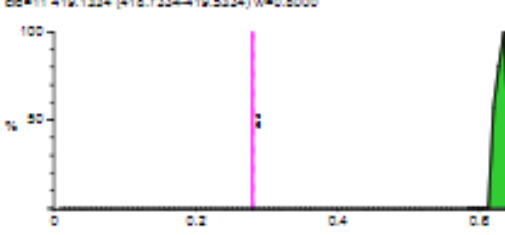

wart4 Hase Pask 1 g0.3s8 min M g(†) E

4.3E06 onts

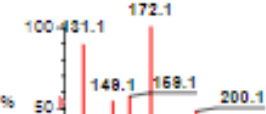

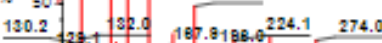
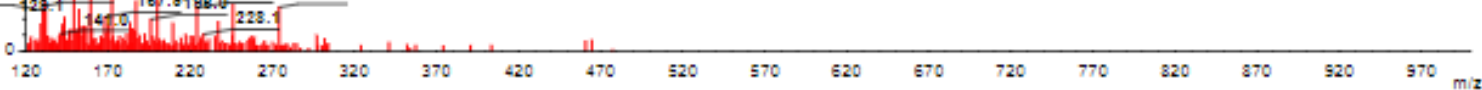

Pask 2 g0.931 mln M $3(+) E B$

2.8E07 onts
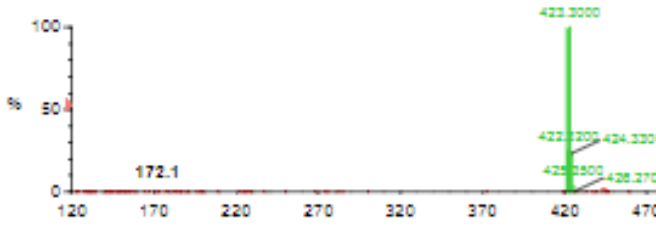
Compound 8f:

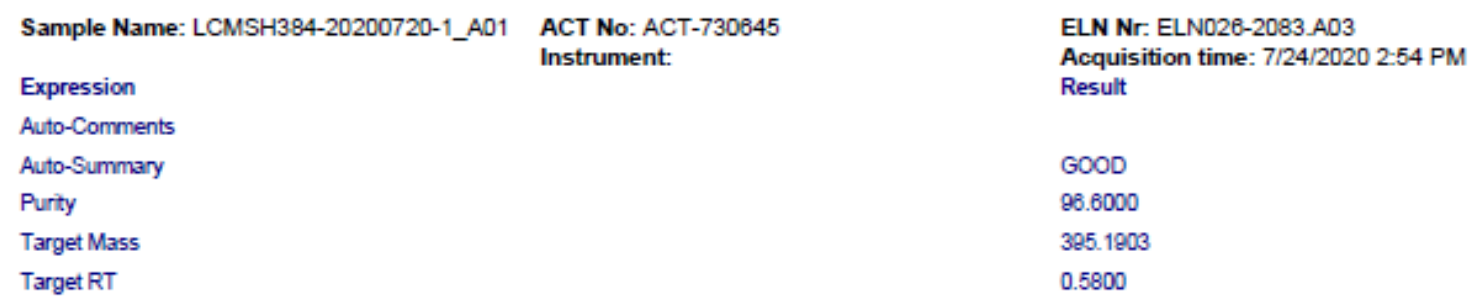

UV214 [D.1E00 ]
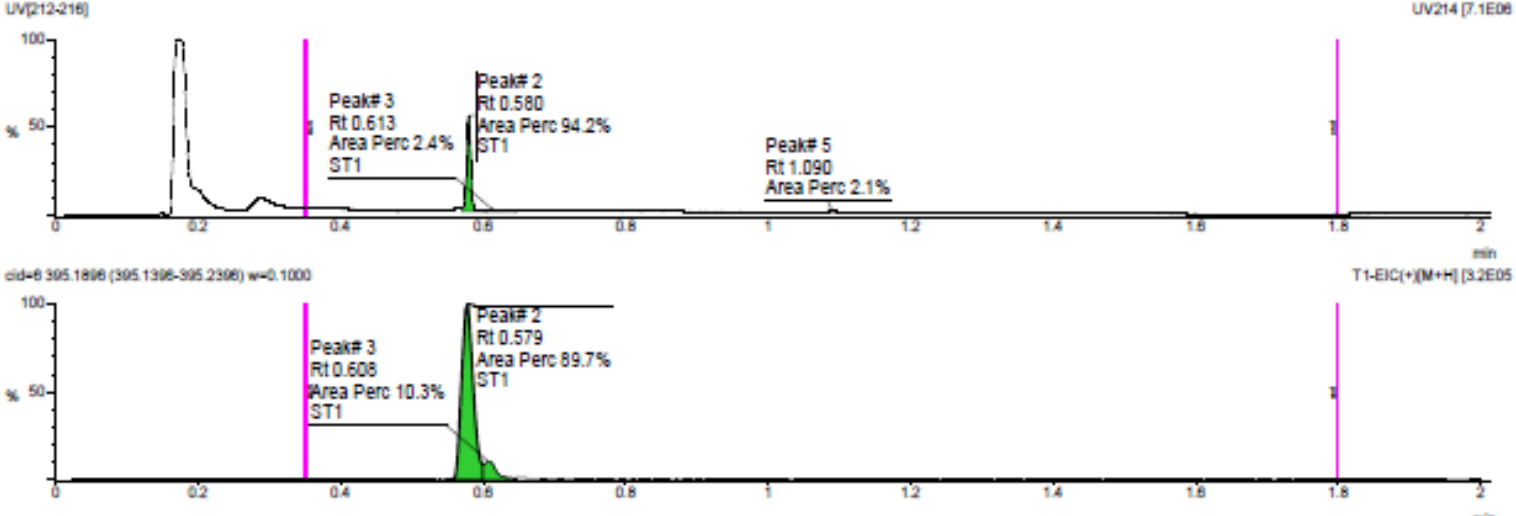

Poak 190.000 min $\mathbf{m} 3(+)$ Es

84188.8 onte

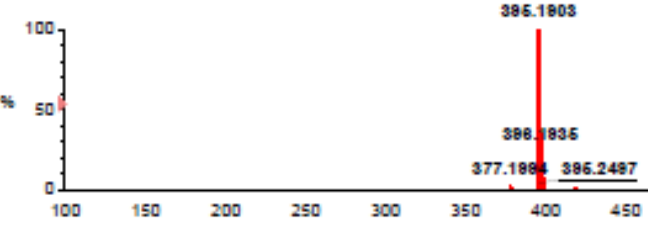

Poak 2g0.579 min M3(+) Es

2.3E06 onte
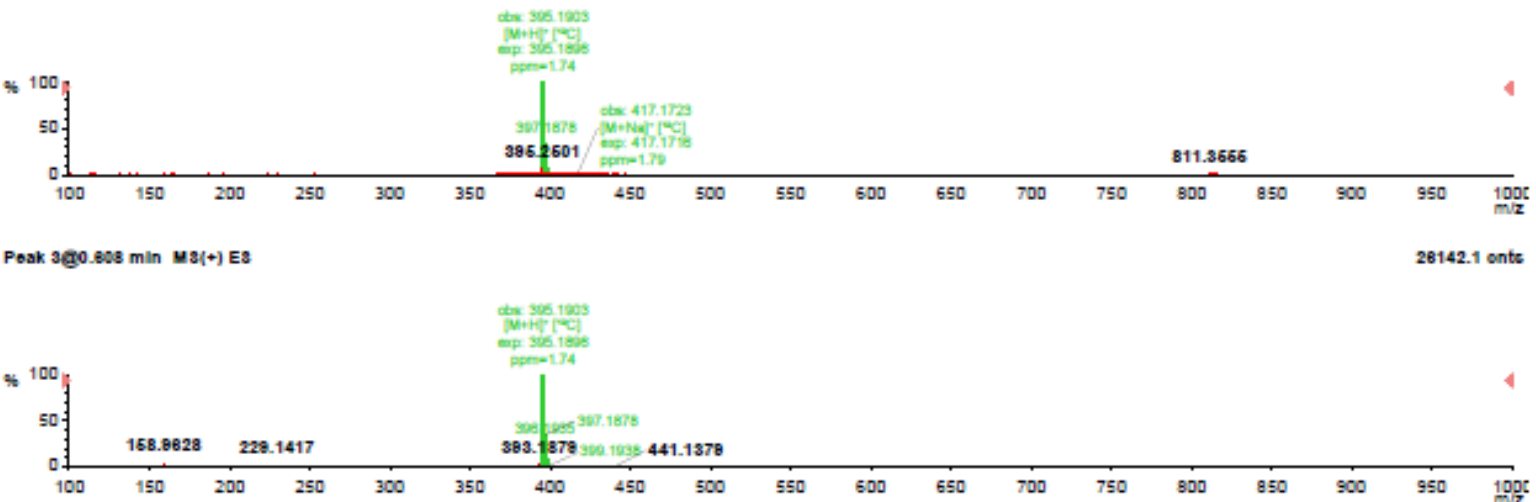
Compound 8g:

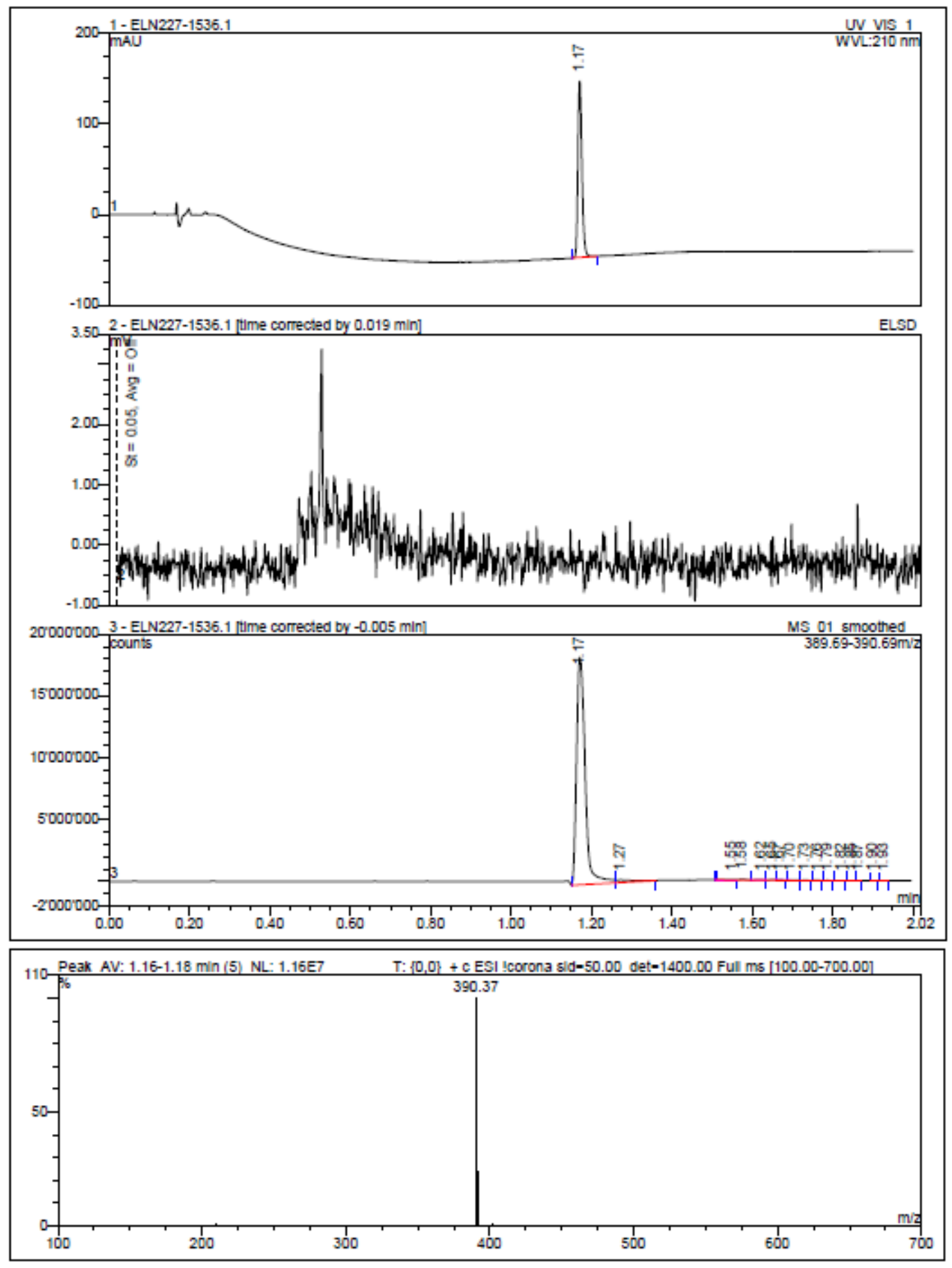


Compound 8h:

3: UV Detector: 212_216

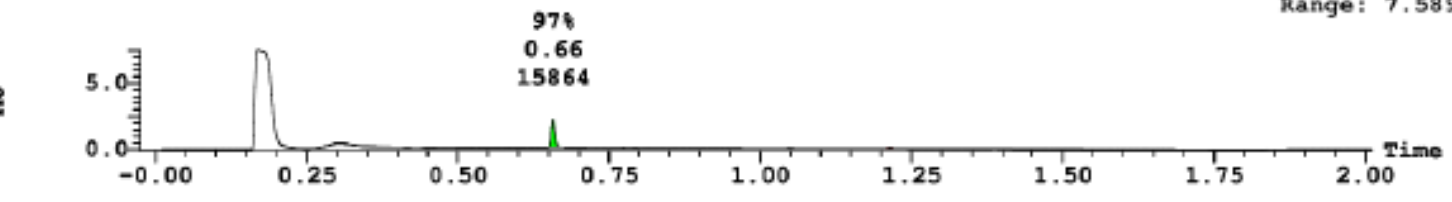

足

1: MS2 ES+ :394.201+372.209 1.0000Da Smooth (SG, 2x1)

$7.3 e+007$

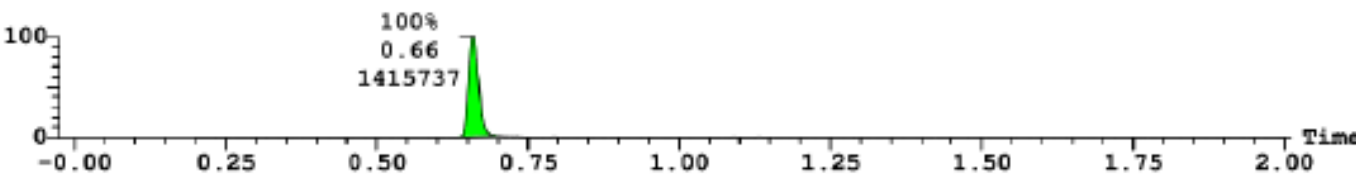

2: MS2 IS- :416.201+370.193 1.0000Da Smooth (SG, 2x1)

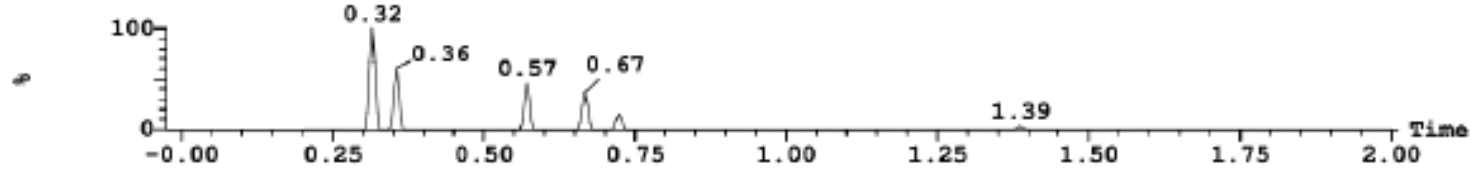

(2) ELSD Signal $100 \%$

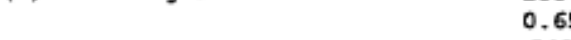

Range: 34,900

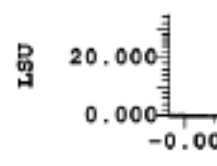

48

1 : MS2 ES+

1: (Time: 0.65$)$ Combine $(112: 117-(94: 97+139: 142))$

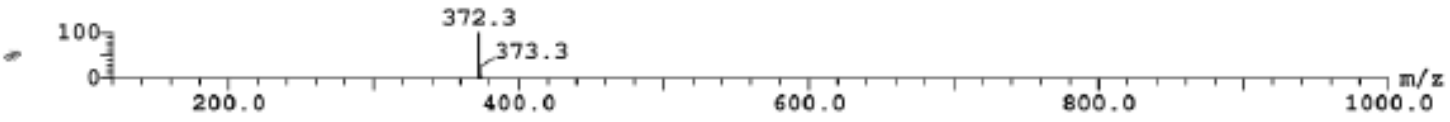


Compound 8i:

Sample Name: LCMS384-20200803-5_K23 ACT No: ACT-773703

Instrument: ICHALW-DLO0012-TQD

\section{Expression}

Auto-Comments

Auto-Summary

Purity

Target Mass

Target RT

บง/212216!

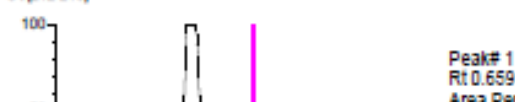

*50-1

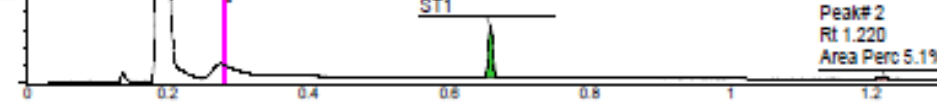

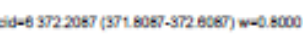

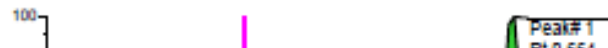

${ }^{100} 7.1$ Pealis:

Area Perc $100.0 \%$

$x$ 50-
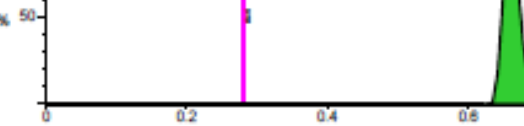

odd-11 370.1931 (360 7991-370.5691) w-0.8000

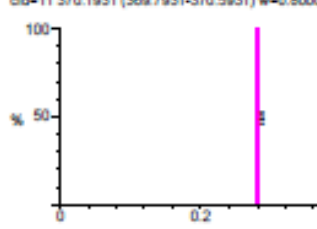

08
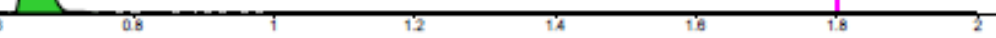

$\min _{\text {T1-EICFIIN-HIII. }}$

Poak 190.694 min $\mathbf{M} 3(+)$ Es

3.0E0 onte

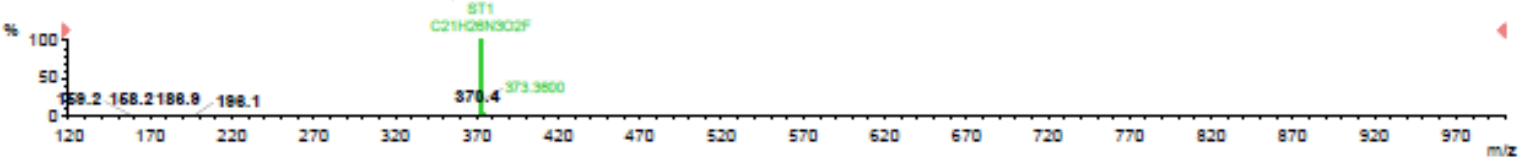


Compound 8j:

3: UV Detector: 212_216

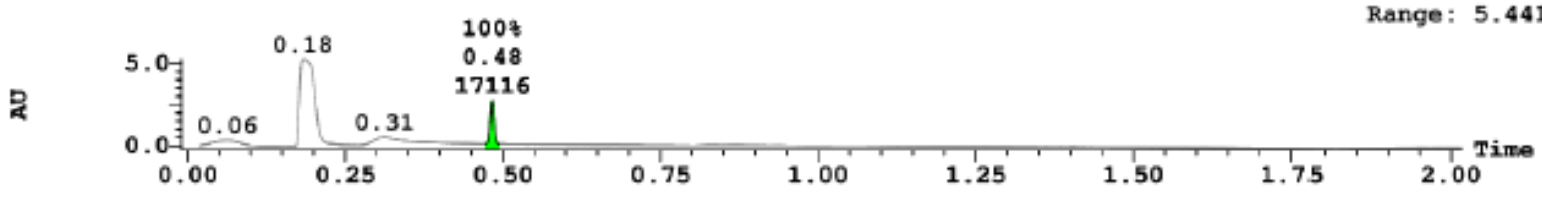

1: MS ESt : $355.2131 .0000 \mathrm{Da} \quad \begin{array}{r}1008 \\ 0.48\end{array}$

7. 1 e+00\%

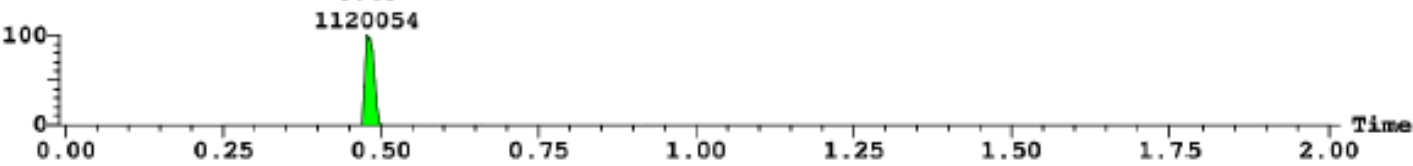

2: MS ES- :353.198 1.0000Da

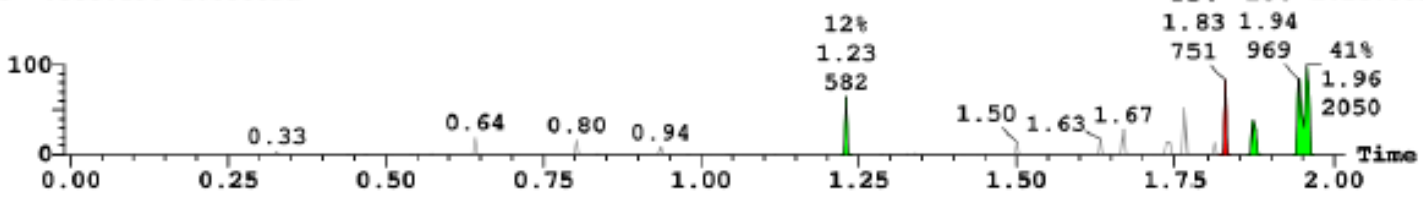

(1) ELSD Signal

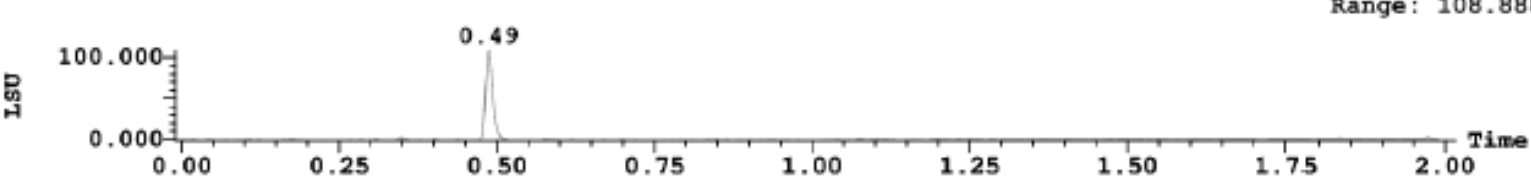

1: (Time: 0.48$)$ Combine $(55: 59-(35: 38+80: 83))$

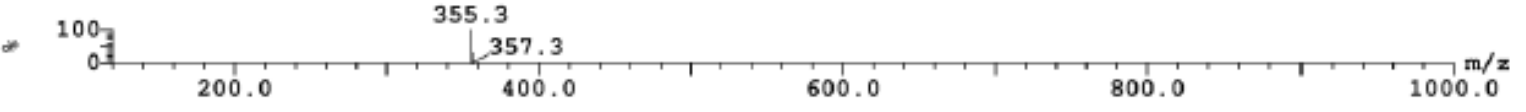


Compound 9a:

Sample Name: LCMS384-20191018-2_E05

\section{Expression}

Auto-Comments

Auto-Summary

Purity

Target Mass

Target RT

UV[212.216]

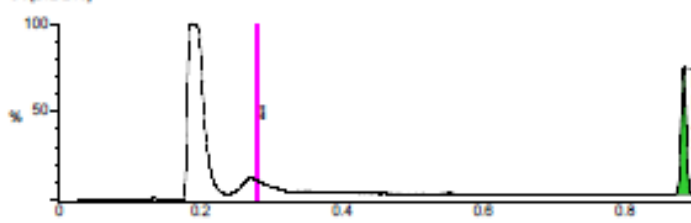

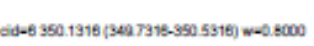

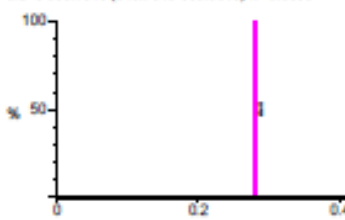

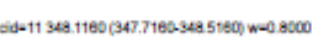

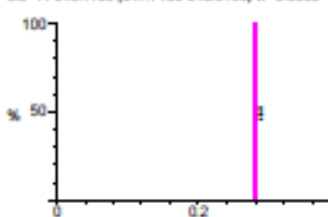

ACT No: IDOR-1119-5082

Instrument: ICHALW-DL00021-SQD

ELN Nr: ELN057-1675.1

Acquisition time: 10/18/2019 3:42 PM

Result

GOOD

99.0000

350.3000

0.8830

UV214 [5.EEO ]

Area Perc $99.0 \%$

ST

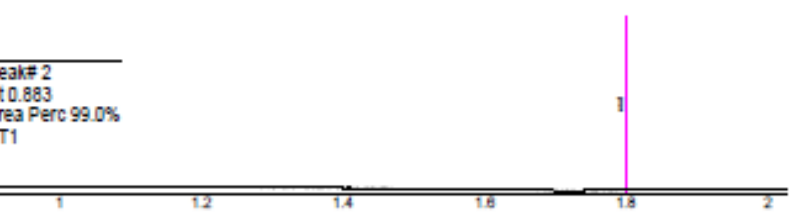

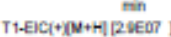

\section{Peakit: 2}

Rt 0.880

Area Perc $100.0 \%$

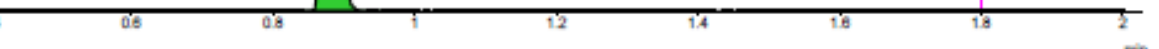

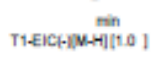

Poak 1 ggo. $652 \mathrm{~min}$ Ms(+) Es

84068.7 onte

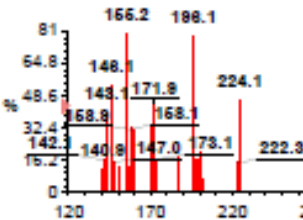

Poak $290.890 \mathrm{mln}$ Mal+) Es

1.7507 onte

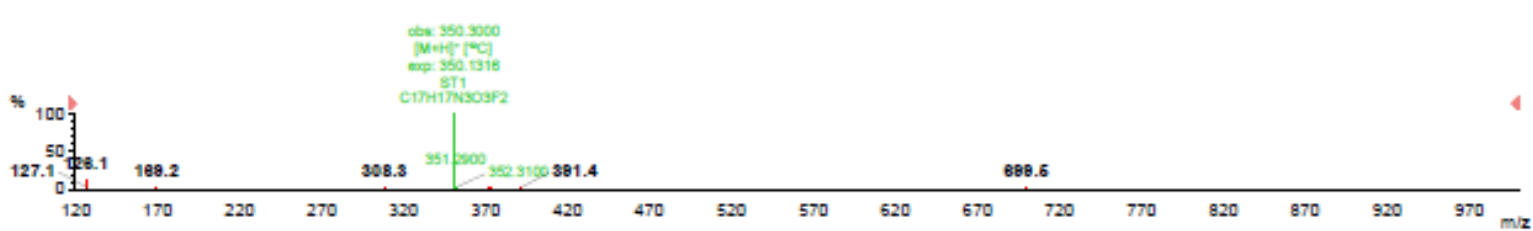


Compound 9b:

3: UV Detector: 212_21

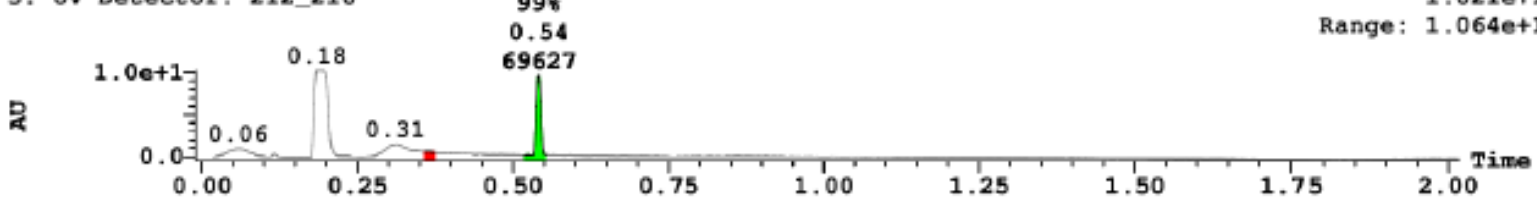

1: MS ES+ : 308.121 1.0000Da $\quad \begin{aligned} & 100 \% \\ & 0.54\end{aligned}$

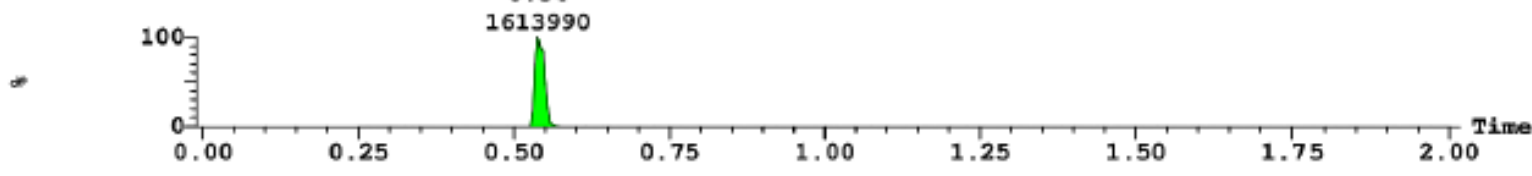

2. MS ES- $.306,105,1.00000$

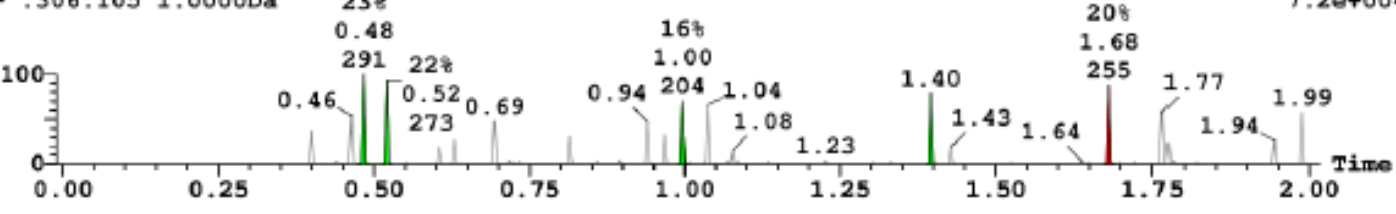

(1) ELSD Signal

0.5

郘 $\quad 40.000 .000-3$

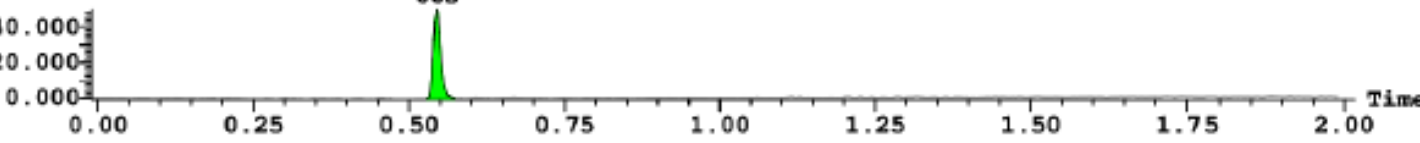

5: (Time: 0.54$)$ Combine $(70: 74-(49: 52+97: 100))$

+ $\quad 007$

308.2

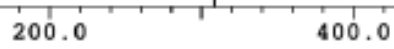


Compound 9c:

Sample Name: LCMSP384-20200528-1_C11 ACT No: ACT-735356

Instrument: ICHALW-DL00021-SQD

Expression

Auto-Comments

Auto-Summary

Purity

Target Mass

Target RT

uv[212216]

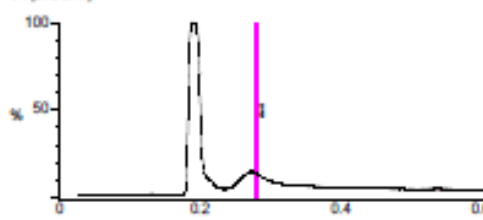

Fit $0.02000 \%$

ST
ELN Nr: ELN028-1200.B01

Acquisition time: 5/28/2020 10:29 AM Result

GOOD

98.2000

376.4000

0.6200

UV214 [4.4EOS ]

oid-6 378. 1837 (375 7837-376 5837) w-0.5000

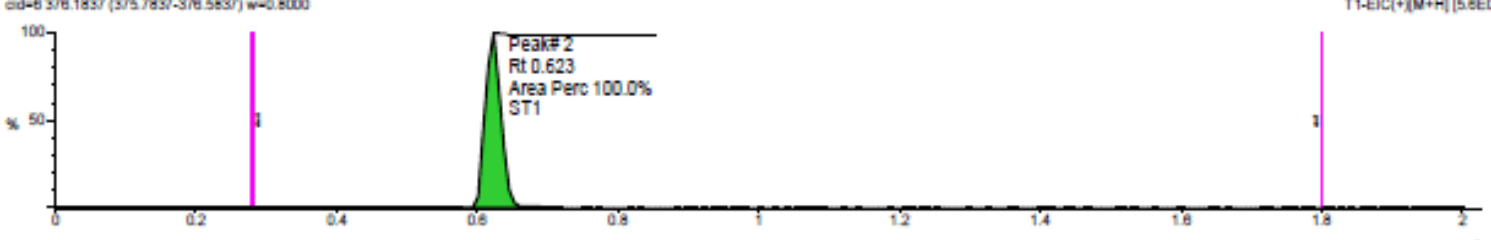

oid-11 374.1650 (9797860-374.5880) w-0.8000

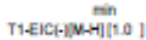

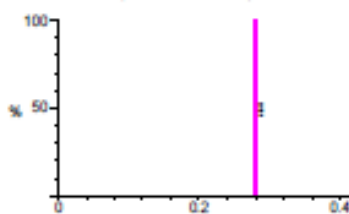

Poak 190.638 min us(+) Es

$1.6 \mathrm{E} 06$ onte

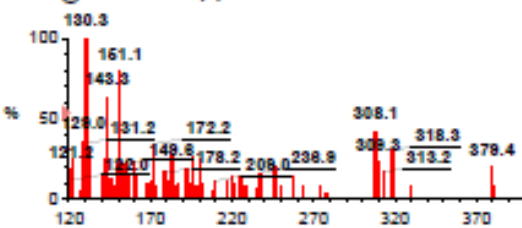

Poak 2 (90.623 min $\mathbf{M} 3(+)$ Ea

3.8E07 onte

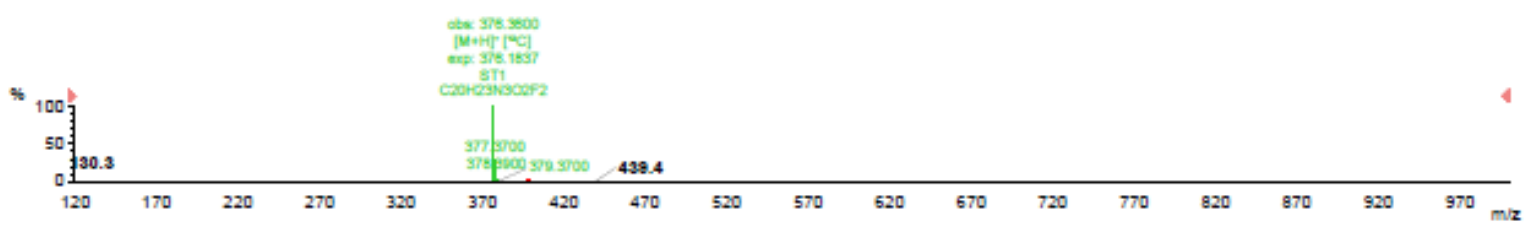


Compound 9d:

Sample Name: LCMSP384-20200528-1_E01 ACT No: ACT-735355

Instrument: ICHALW-DL00021-SQD

\section{Expression}

Auto-Comments

Auto-Summary

Purity

Target Mass

Target RT

บง[212.216]

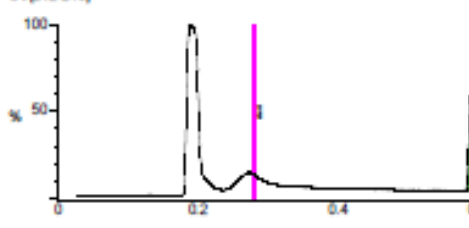

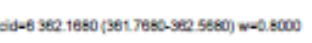

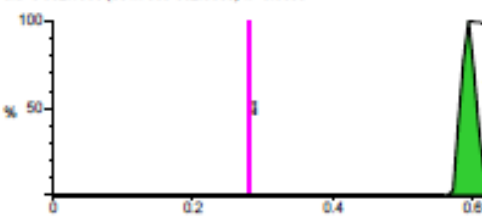

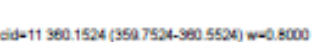

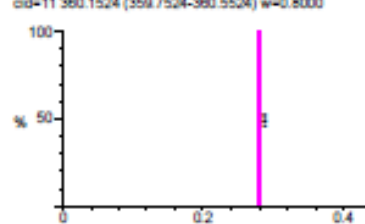

eakn 1

Frea Perc 10000

Area Perc $1000 \%$

L

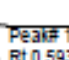

Rit 0.593

Area Perc $100.0 \%$
ELN Nr: ELN026-1200_A0B

Acquisition time: 5/28/2020 8:38 AM Result

GOOD

100.0000

362.3000

0.5910

UV214 [LSEOS ]

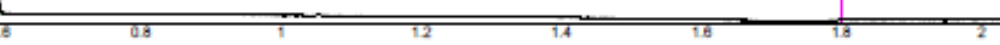

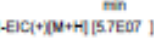

Poak 190.693 min $\mathbf{M} 9(+)$ Es

3.9E07 onte

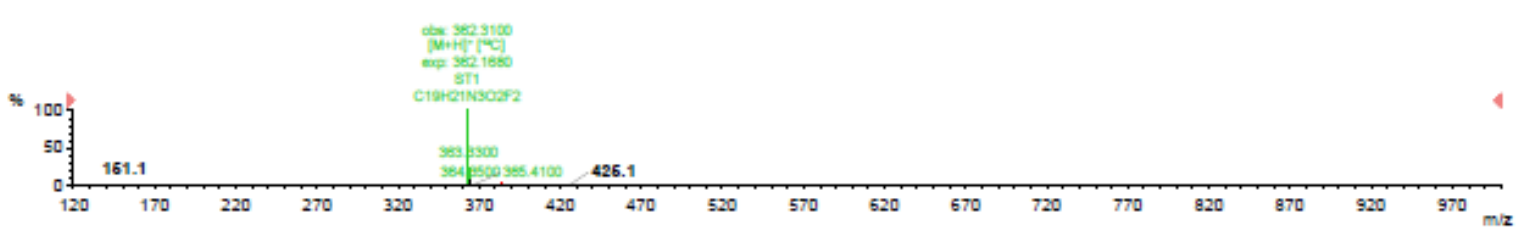


Compound 9e:
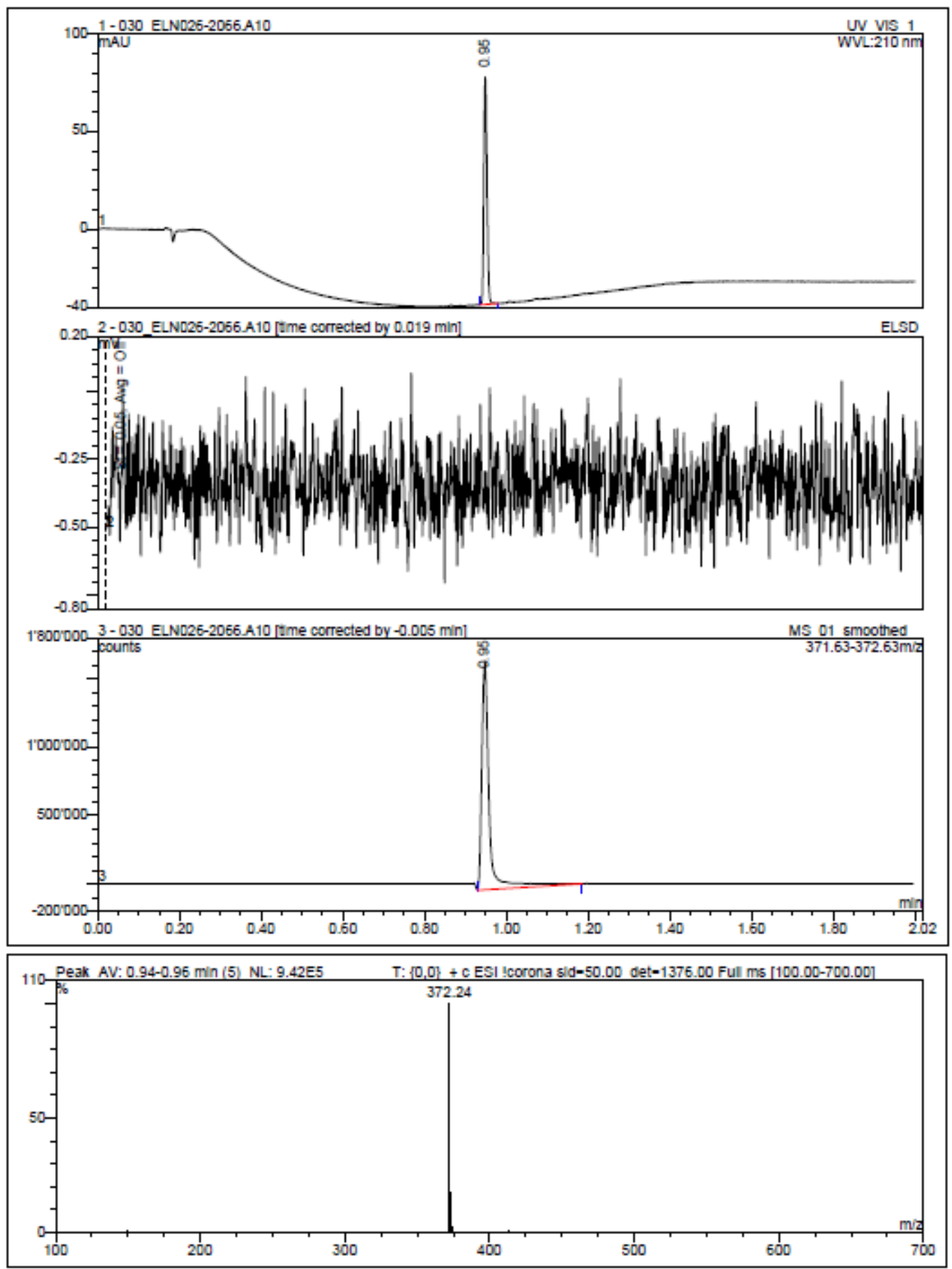
Compound 9f:

3: UV Detector: 212_216

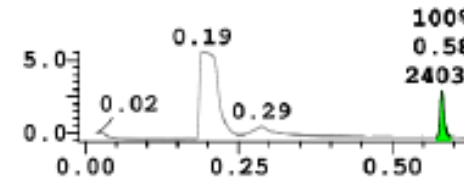

1009
0.58

Range: 6.121

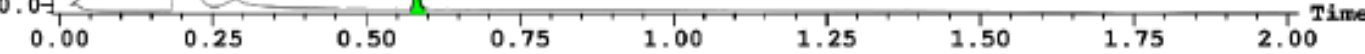

1: MS ES+ :366.163 $1.0000 \mathrm{Da} \quad \begin{aligned} & 100 \% \\ & 0.58\end{aligned}$

$9.3 e+007$

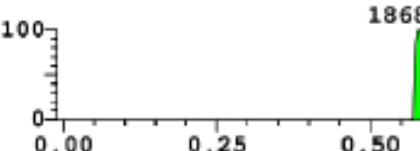

68161
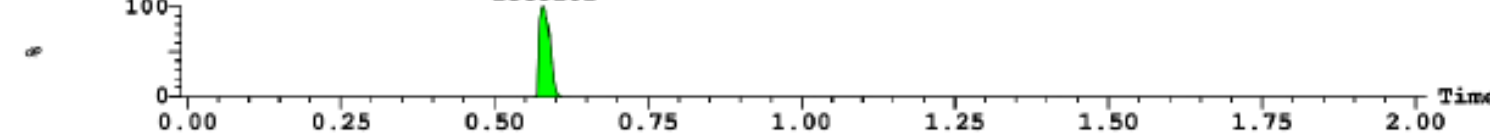

2: MS ES- : 364.147 1.0000Da

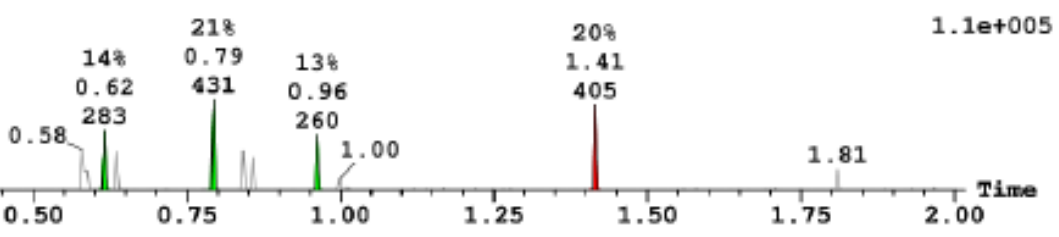

(1) ELSD Signal

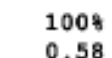

91.348

0.58

Range: 92.019

品 50.000

$0.000 \frac{1}{1}$

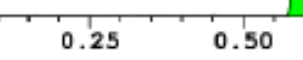

0.75

1.00

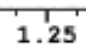

1.50

$2^{\text {Time }}$

2: (Time: 0.58$)$ Combine $(93: 97-(71: 74+119: 122)$ )

1 :MS ES+

8. $2 e+007$

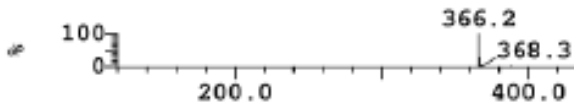




\section{Compound 9g:}

Sample Name: LCMSH384-20200714-1_K01 ACT No: ACT-735364

Instrument: UPLC_SYNAPT1

\section{Expression}

Auto-Comments

Auto-Summary

Purity

Target Mass

Target RT

UV?212216]

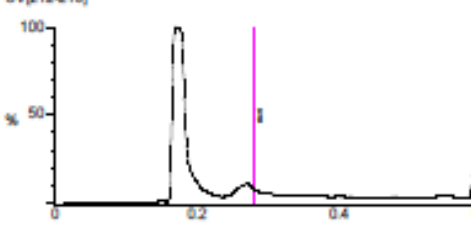

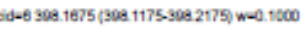

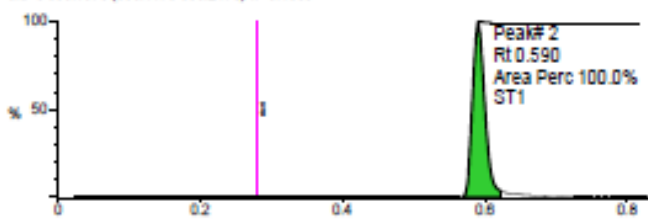

ELN Nr: ELNO26-2066_A07

Acquisition time: $7 / 15 / 2020$ 2-42 PM

Result

GOOD

98.8000

398. 1675

0.5930

Poak 1 go $.331 \mathrm{~min}$ Mal+) Es

1074.8 onte

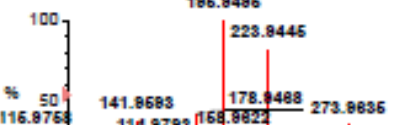

16.976 . 114.9783 .168 .9822

196. 631.8005

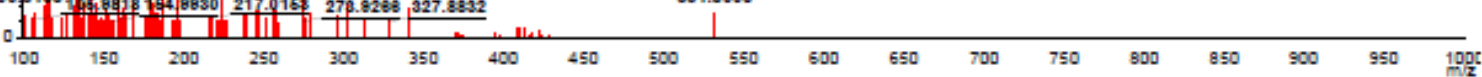

Poak 2 ge. $690 \mathrm{~min}$ M3(+) Es

4.2E06 onte

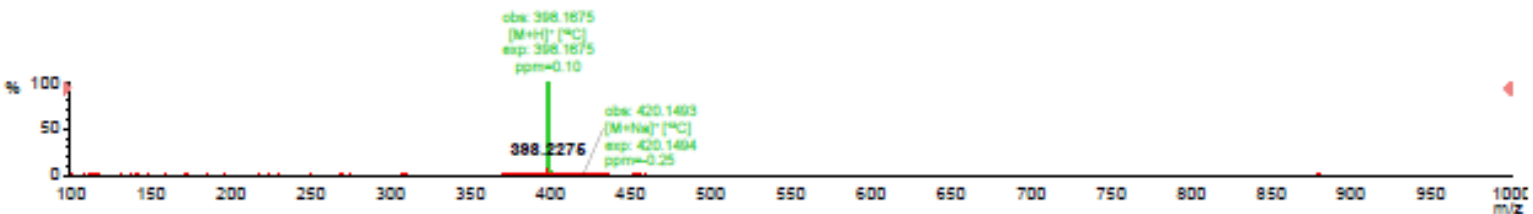


Compound 10a:

3: UV Detector: 212_216

是

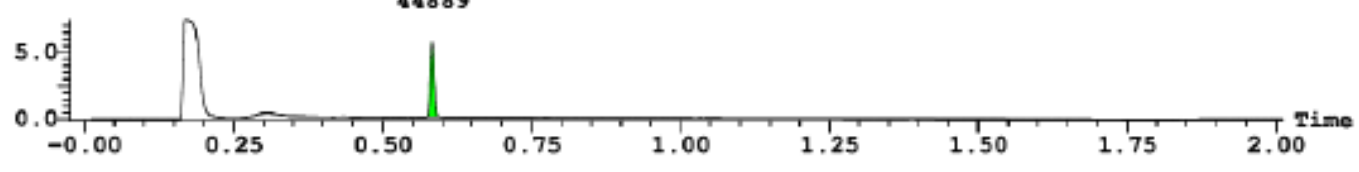

1: MS2 ESt : 411.207+389.215 1.0000Da Smooth (SG, 2x1)

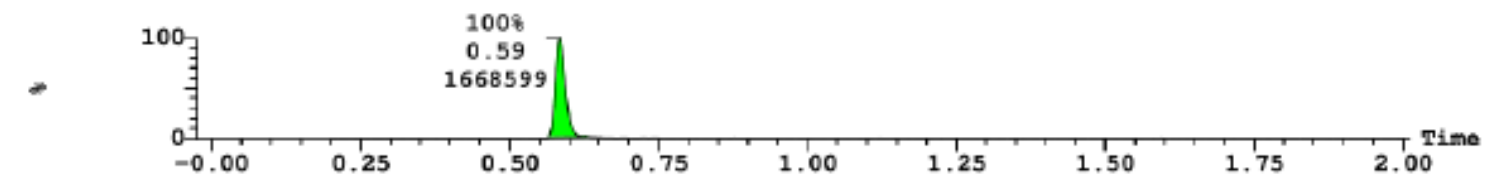

2: MS2 ES- :433.207+387.2 1,0000Da Smooth (SG, 2x1)

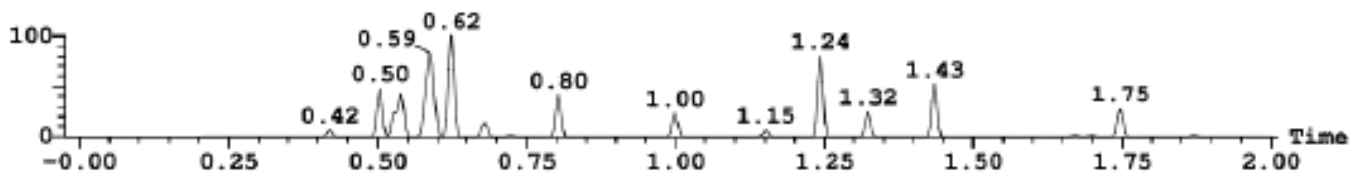

(2) ELSD Signal

103.169

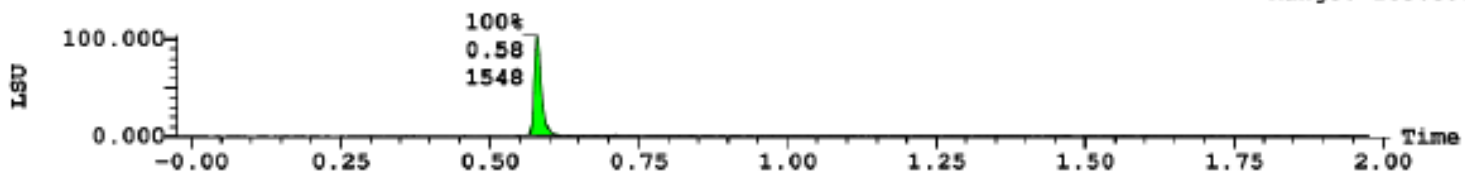

1:(Time: 0.58$)$ Combine $(93: 98-(76: 79+118: 121))$

$1:$ MS2 Es+

$5.5 e+007$

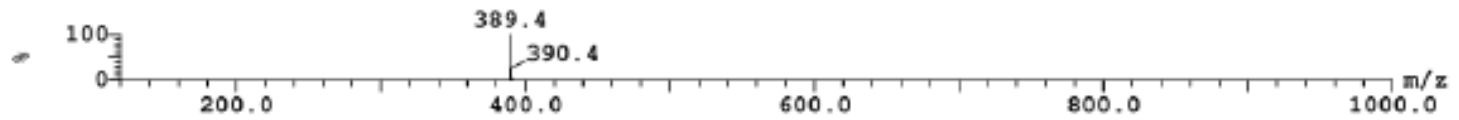


Compound 10b:

3: UV Detector: 212_216

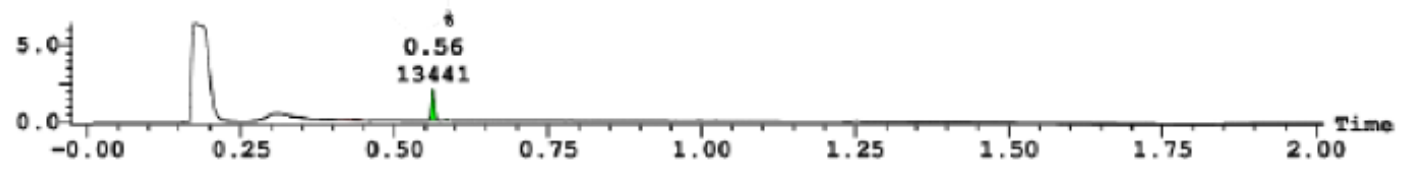

1: MS2 ISt ; 412,203+390,211 1,0000Da Smooth (SG, 2xe1)

5. $8 e+007$

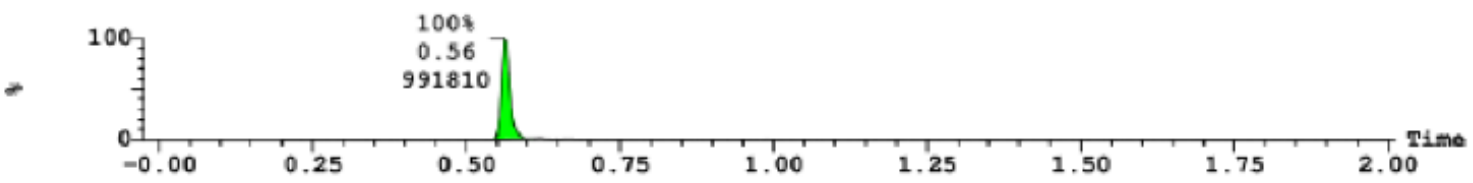

2: MS2 ES- :434.203+388.195 1.0000Da Smooth (SG, 2x1)

$2.5 e+004$

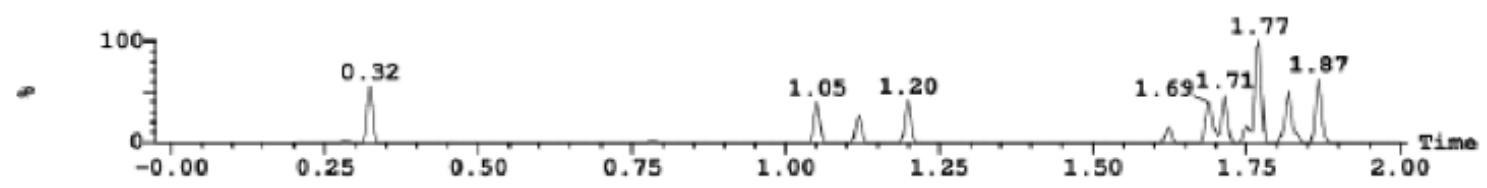

(2) ELSD Signal

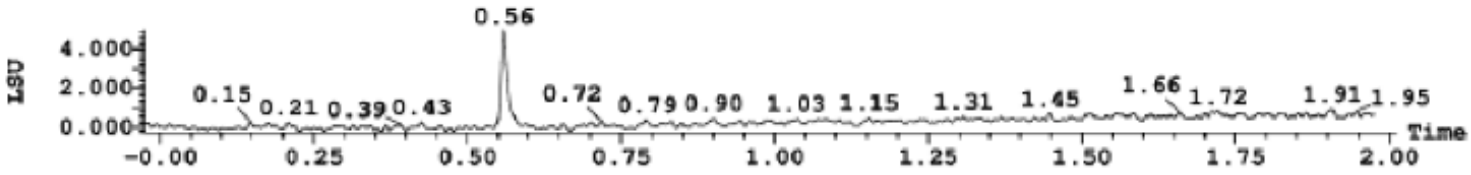

1: (Time: 0.44) Combine (58:63-(37:40+77:80))

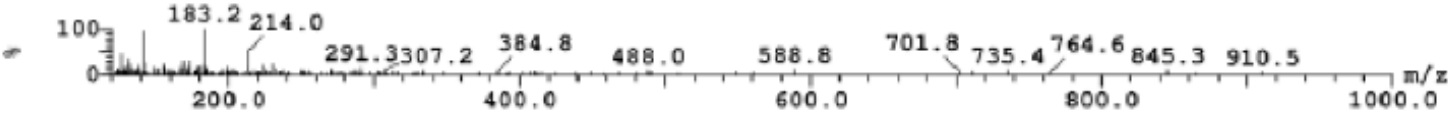

2: (Time: 0.56$)$ Combine (88:94-(72:74+116:118))

1: MS2 ES+

$3.3 e+007$

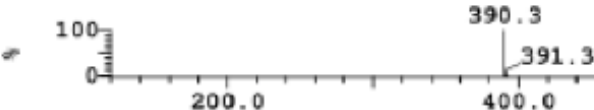

800.0 $1,1,1,1,1000$


Compound 10c:

Sample Name: LCMS384-20200615-4_G05

Expression

Auto-Comments

Auto-Summary

Purity

Target Mass

Target RT

เv[212.216]

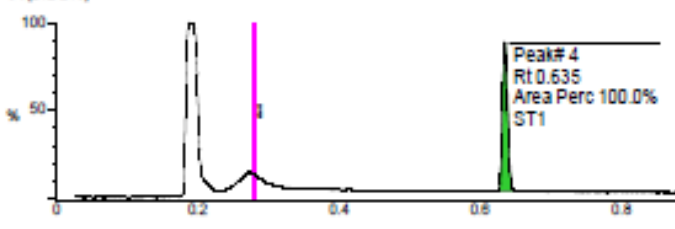

edd-6 300.1906 (962 7005-900 5005) w-0.8000

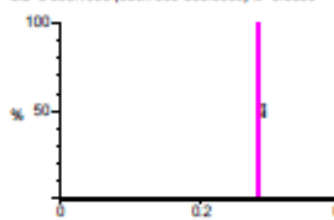

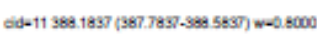

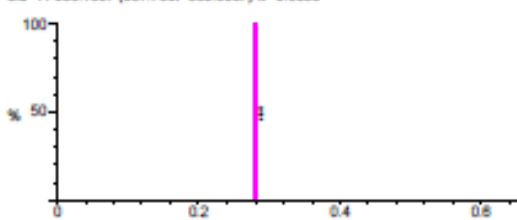

Posk 1 ge. $335 \mathrm{~min}$ M3(+) Es
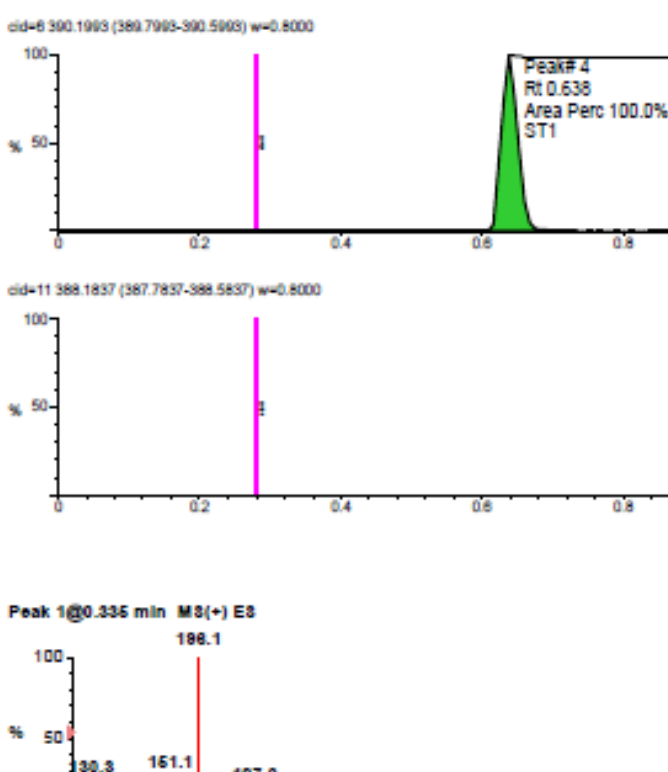

rea Perc 100.0\%

Are
ACT No: ACT-736685

Instrument: ICHALW-DL00021-SQD

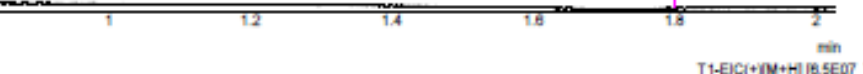

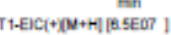

1.130 .3 161.1 6.5 197.0 227.1

$282.300 .5 \quad 381.4$

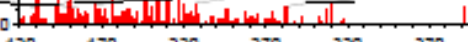

Poak 490.ess min Wa(+) Es

ELN Nr: ELN227-1496.1

Acquisition time: $6 / 15 / 2020$ 5:52 PM

Result

GOOD

100.0000

380.4000

0.6350

UV214 [43EDS]

$4.6 E 07$ onte

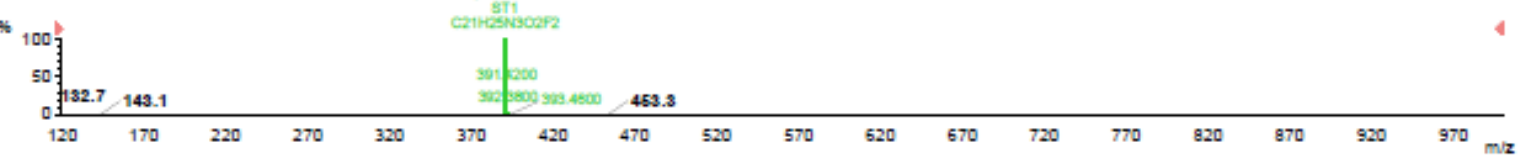


Compound 10d:

Sample Name: LCMSP384-20200528-1_G01 ACT No: ACT-736699

Instrument: ICHALW-DL00021-SQD

\section{Expression}

Auto-Comments

Auto-Summary

Purity

Target Mass

Target RT

Uง[212.216]

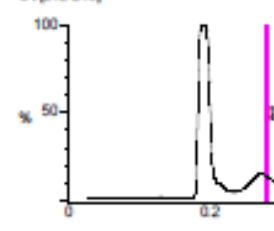

oid-6 301.1946 (900 7948-591.5046) w-0.800

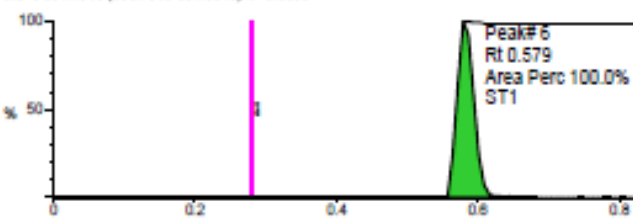

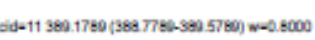

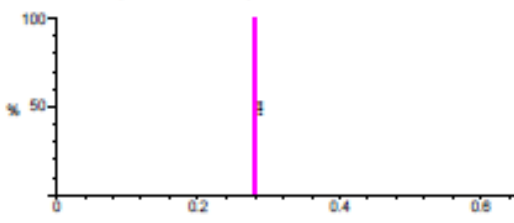

ELN Nr: ELN008-1108.G02

Acquisition time: 5/28/2020 8:41 AM

Result

GOOD

97.2000

391.4000

0.5800

UV214[LAEOS ]

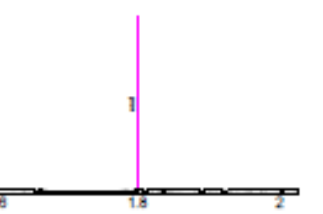

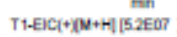

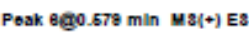

3.8E07 onte

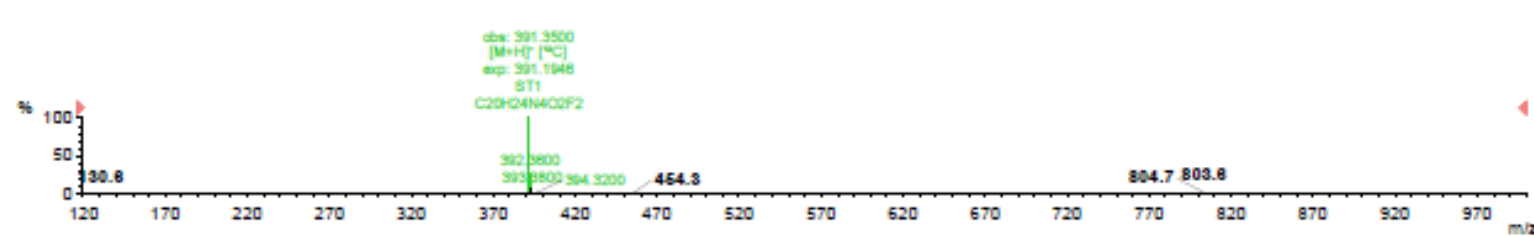


Compound 10e:

Sample Name: LCMS384-20191128-1_E01 ACT No: ACT-761438

\section{Expression}

Auto-Comments

Auto-Summary

Purity

Target Mass

Target RT

UV[212-216]
Instrument: ICHALW-DL00021-SQD

ELN Nr: ELN057-0939

Acquisition time: 11/28/2019 2:10 PM Result

GOOD

100.0000

391.3000

0.5700
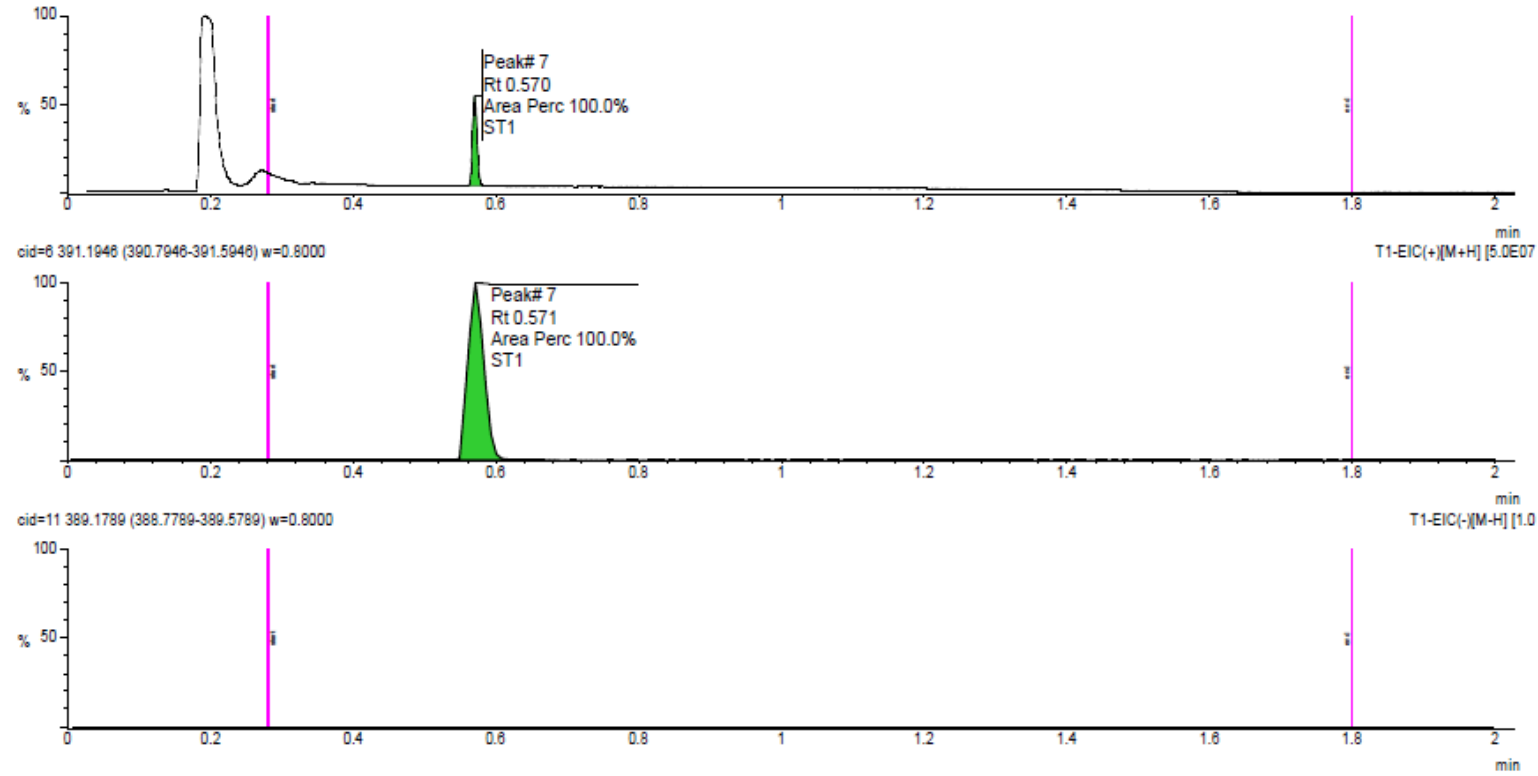

Peak 7@0.571 min MS(+) ES

3.3E07 cnts

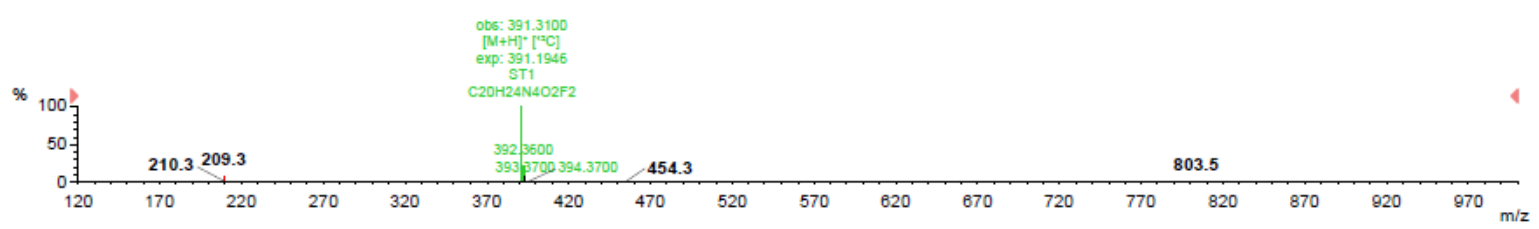


Compound 10f:
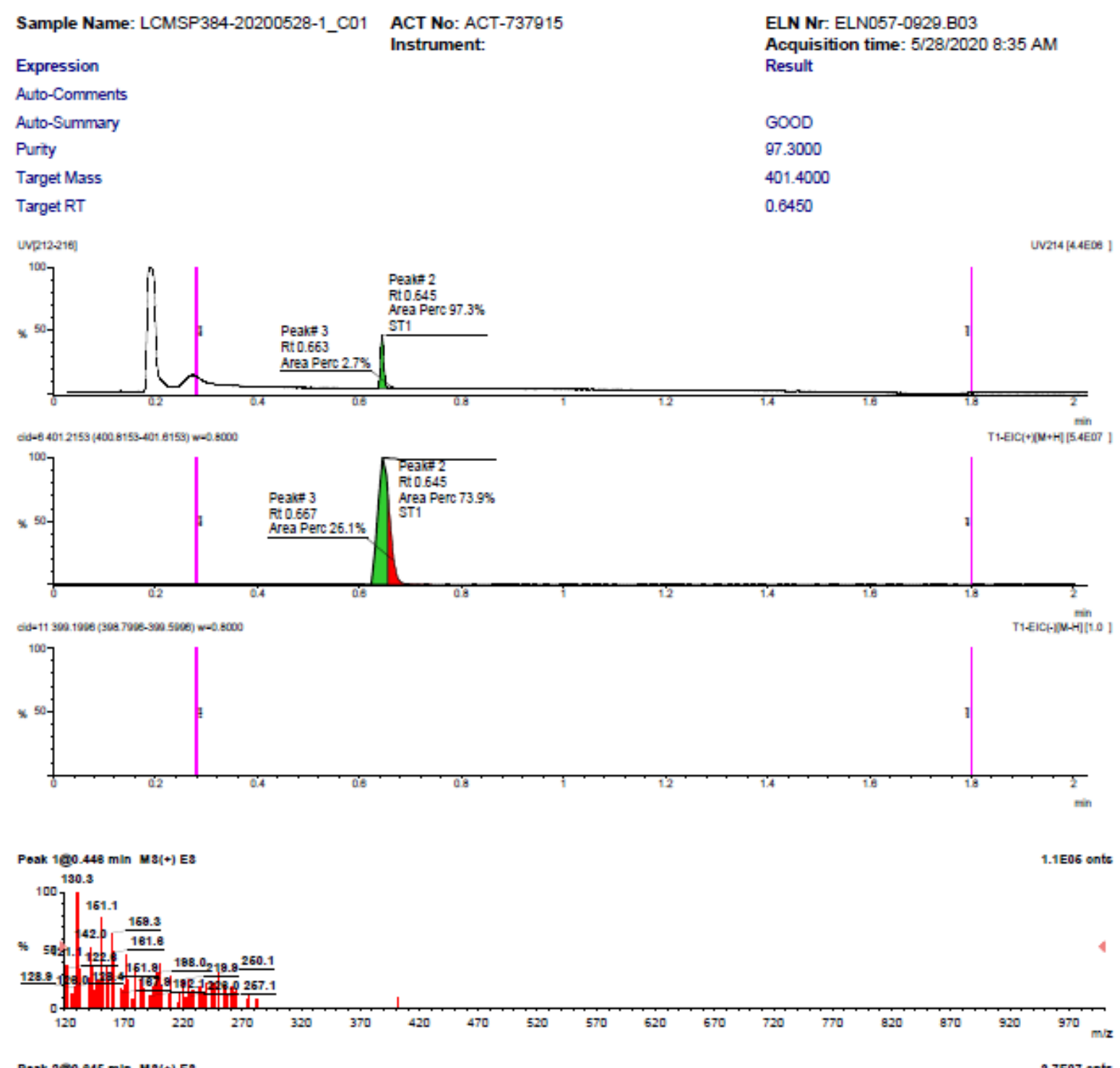

Poak 2 a00.045 min us/+1 Es

$3.7 E 07$ onte

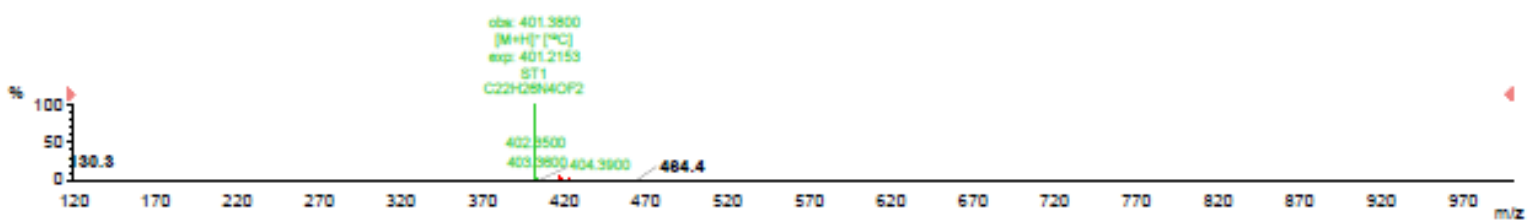

Posk 12 ge. $578 \mathrm{~min}$ Ms(+) Es

48084.0 onte

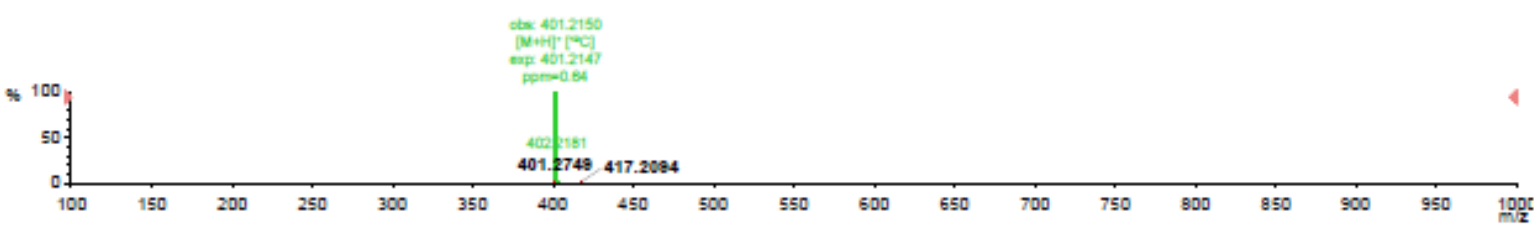


Compound 11a:
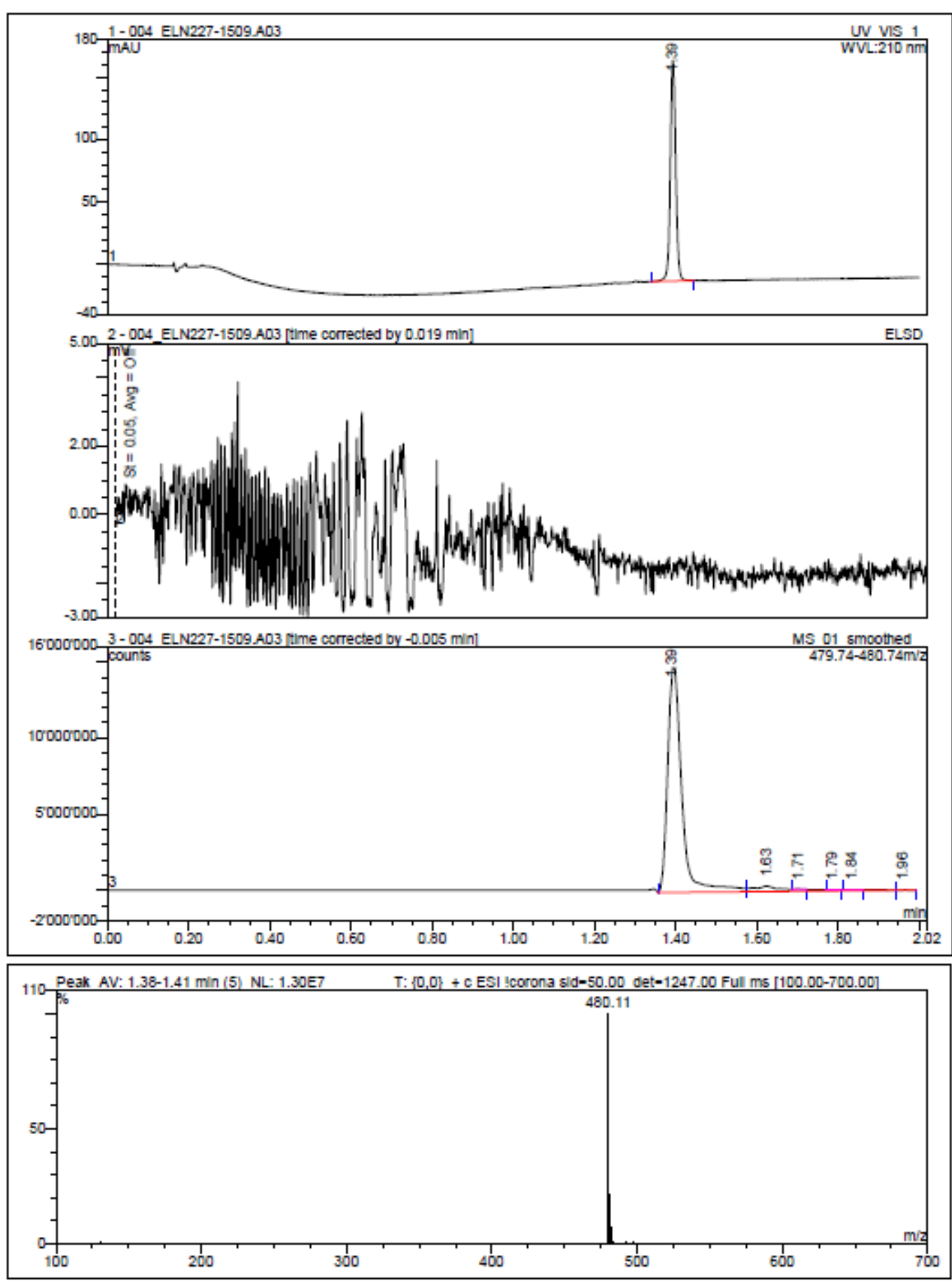
Compound 11b:
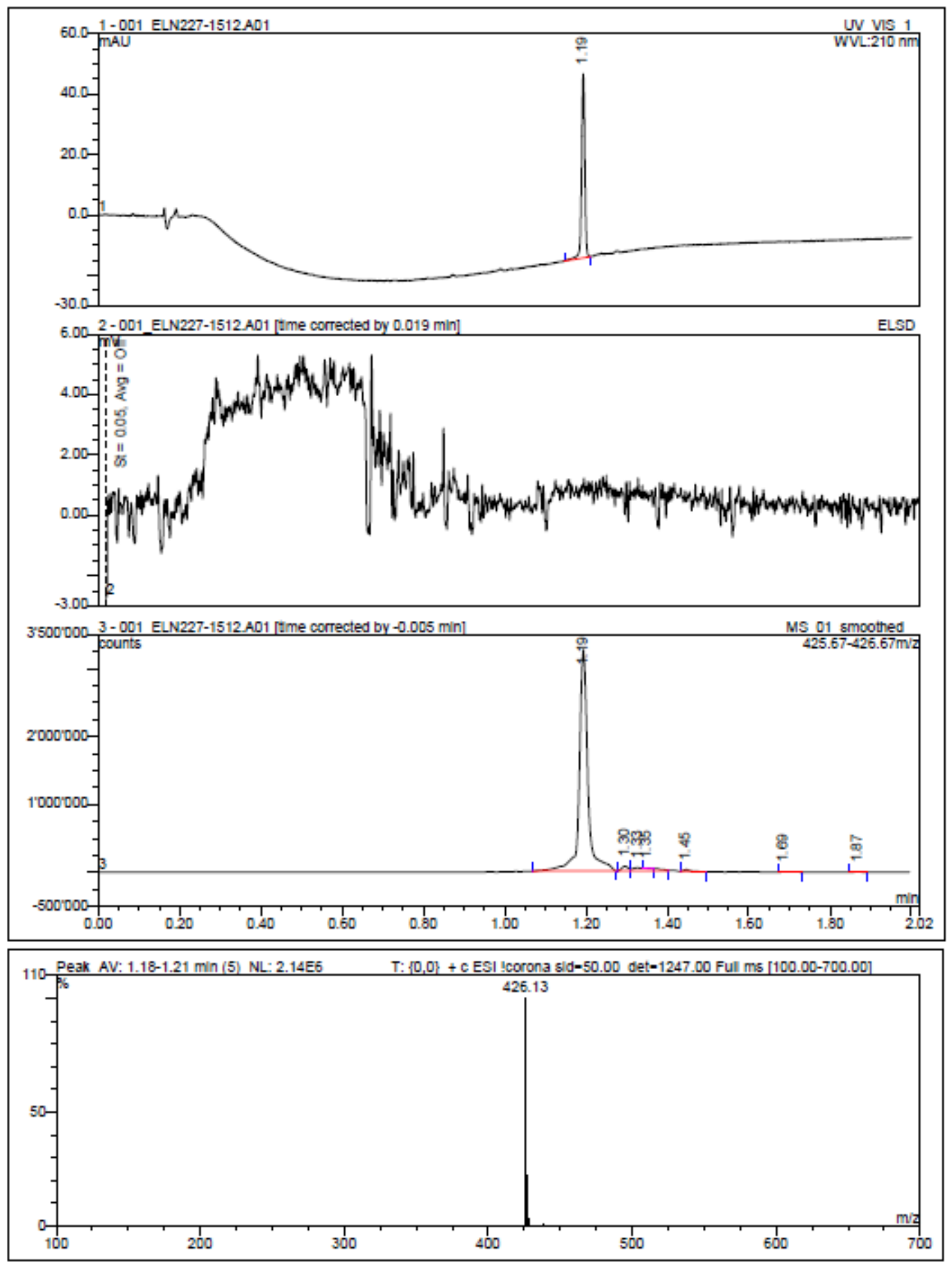
Compound 11c:
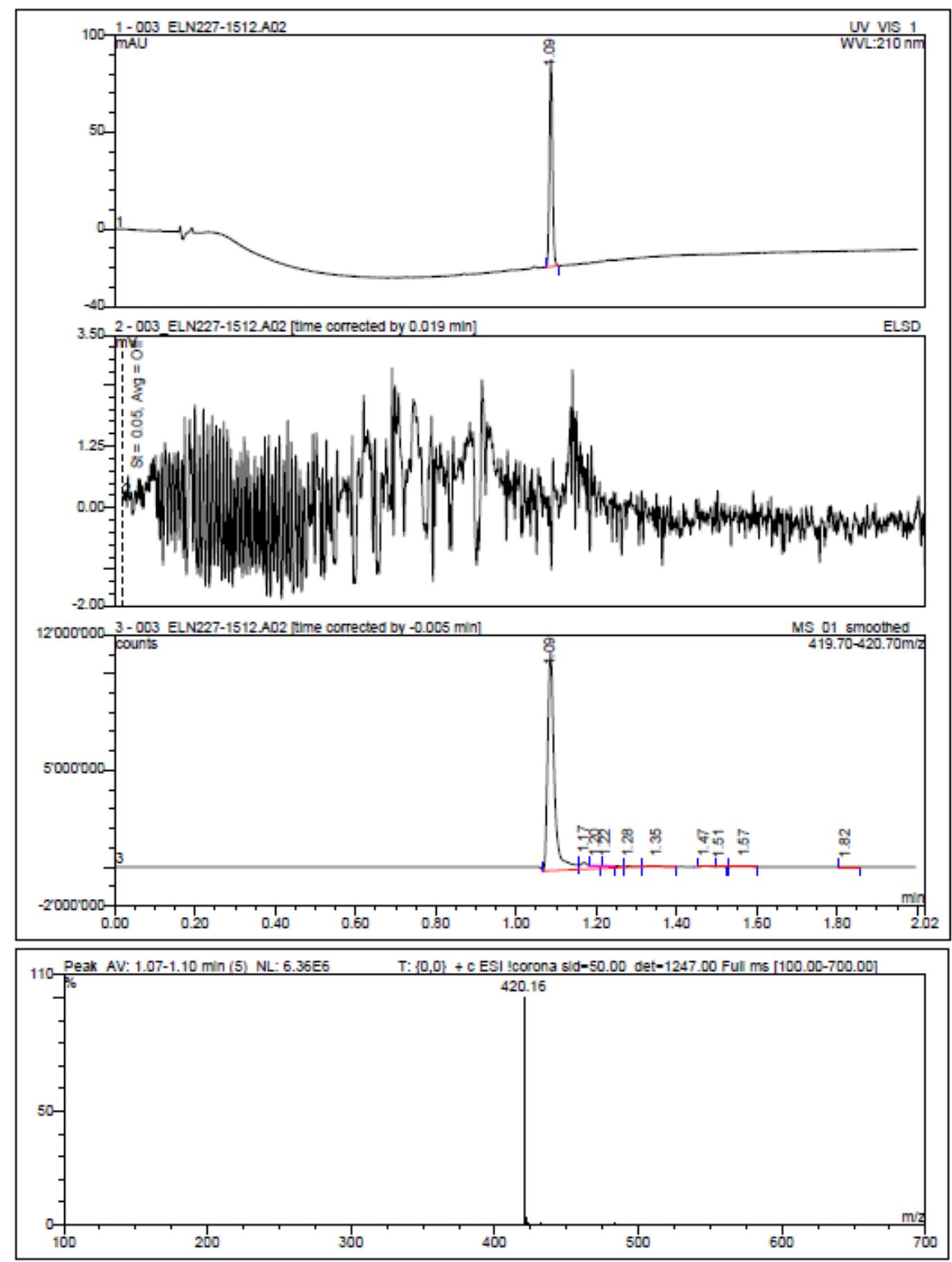
Compound 11d:
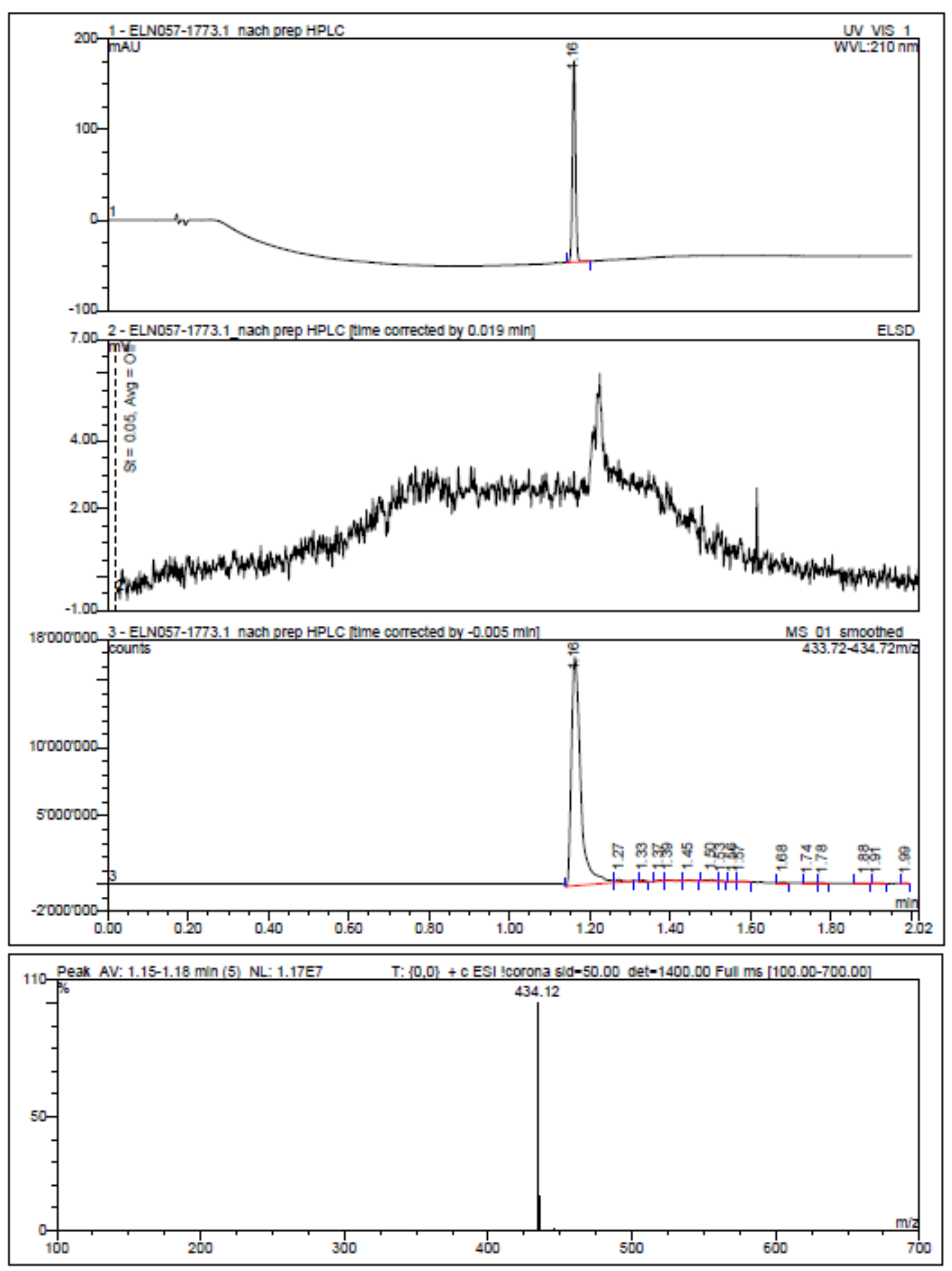
Compound 11e:

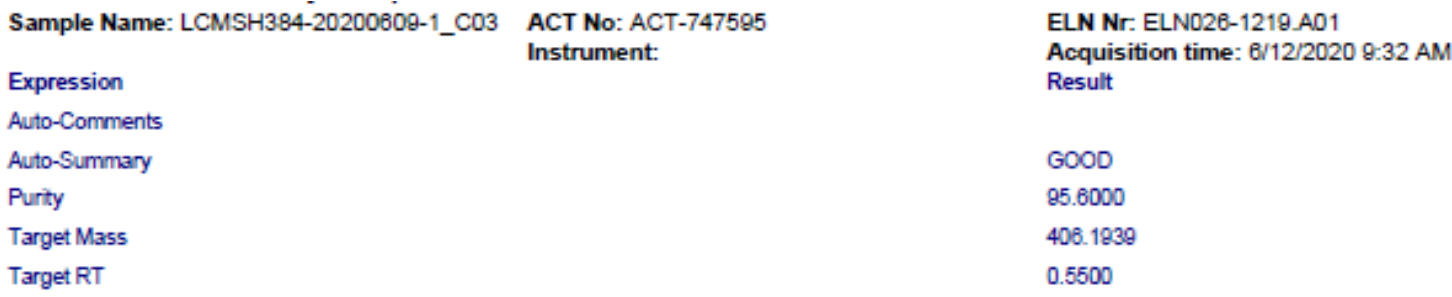

Target Mass

406.1839

Target RT

0.5500

unp12.21

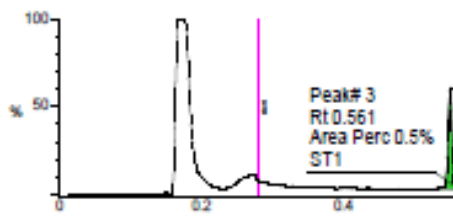

UV214 [B2E00 ]

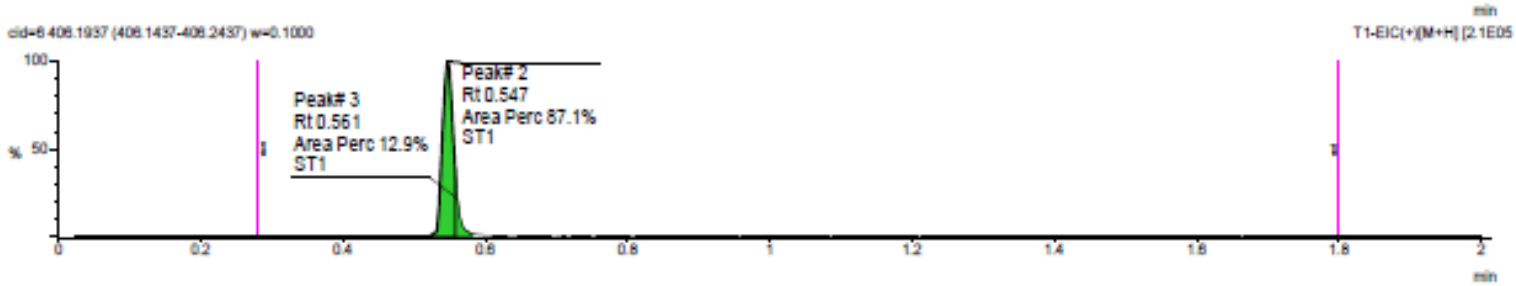

\begin{tabular}{l} 
Rt 0.954 \\
Area Perc 4.4 \\
\hline
\end{tabular}

Aarea Perc 95.1\%

Area Pere $4.4 \%$

Poak 2 ge. 547 min Ma(+) Es

$1.6 \mathrm{E} 06$ onte

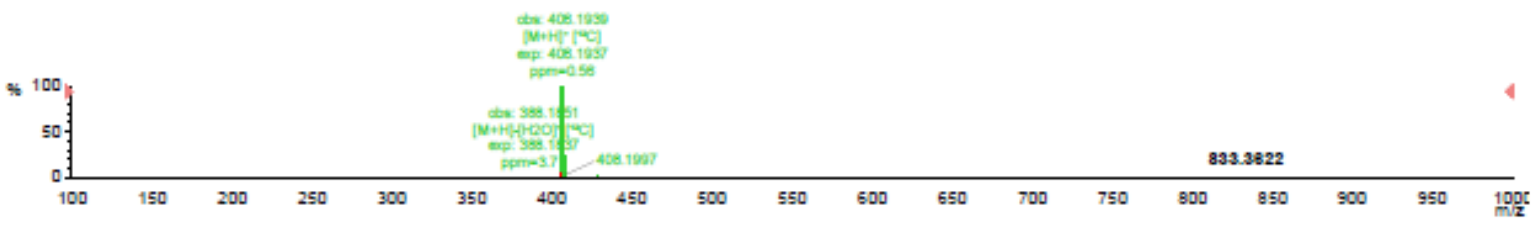


Compound 11f:
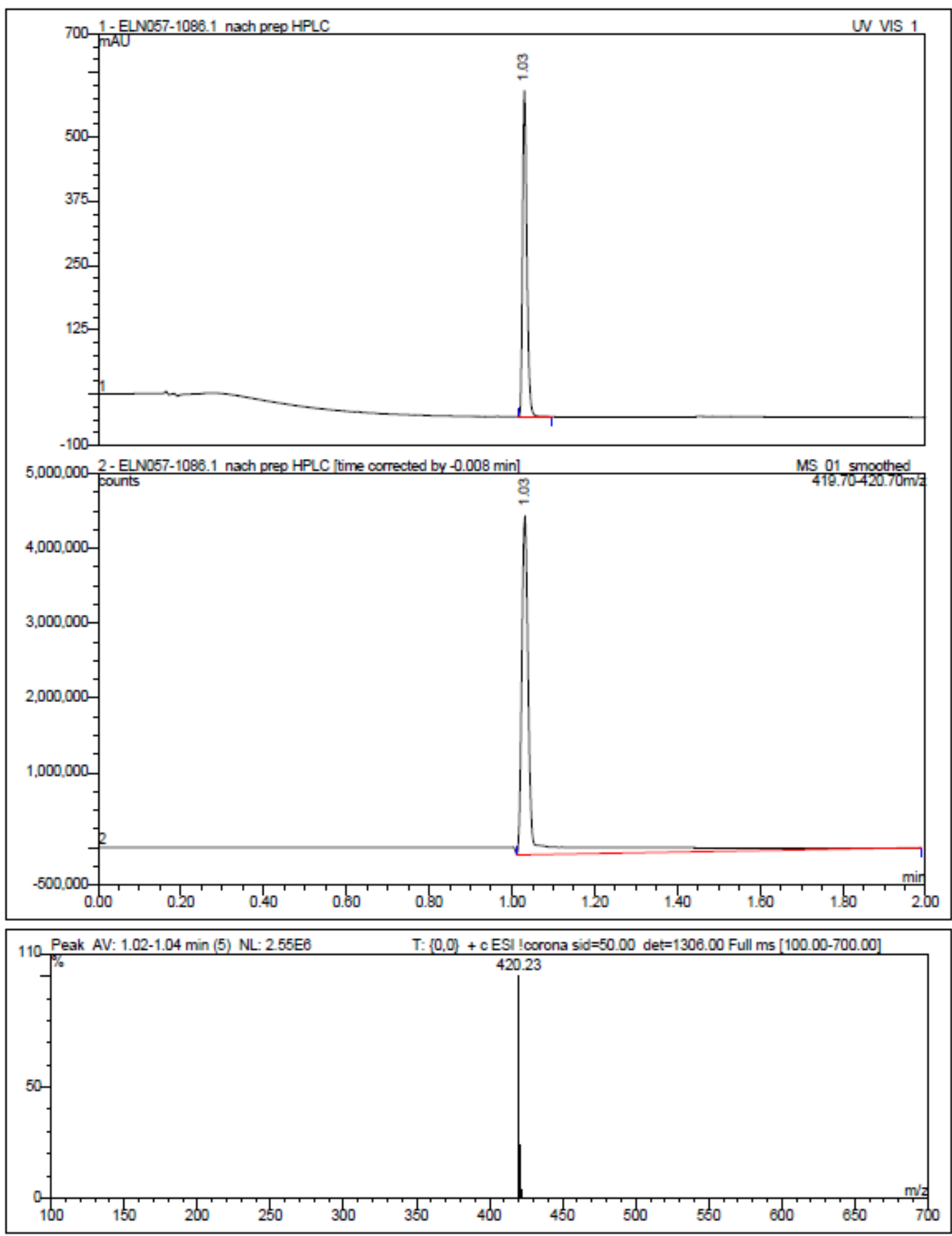
Compound 11g:

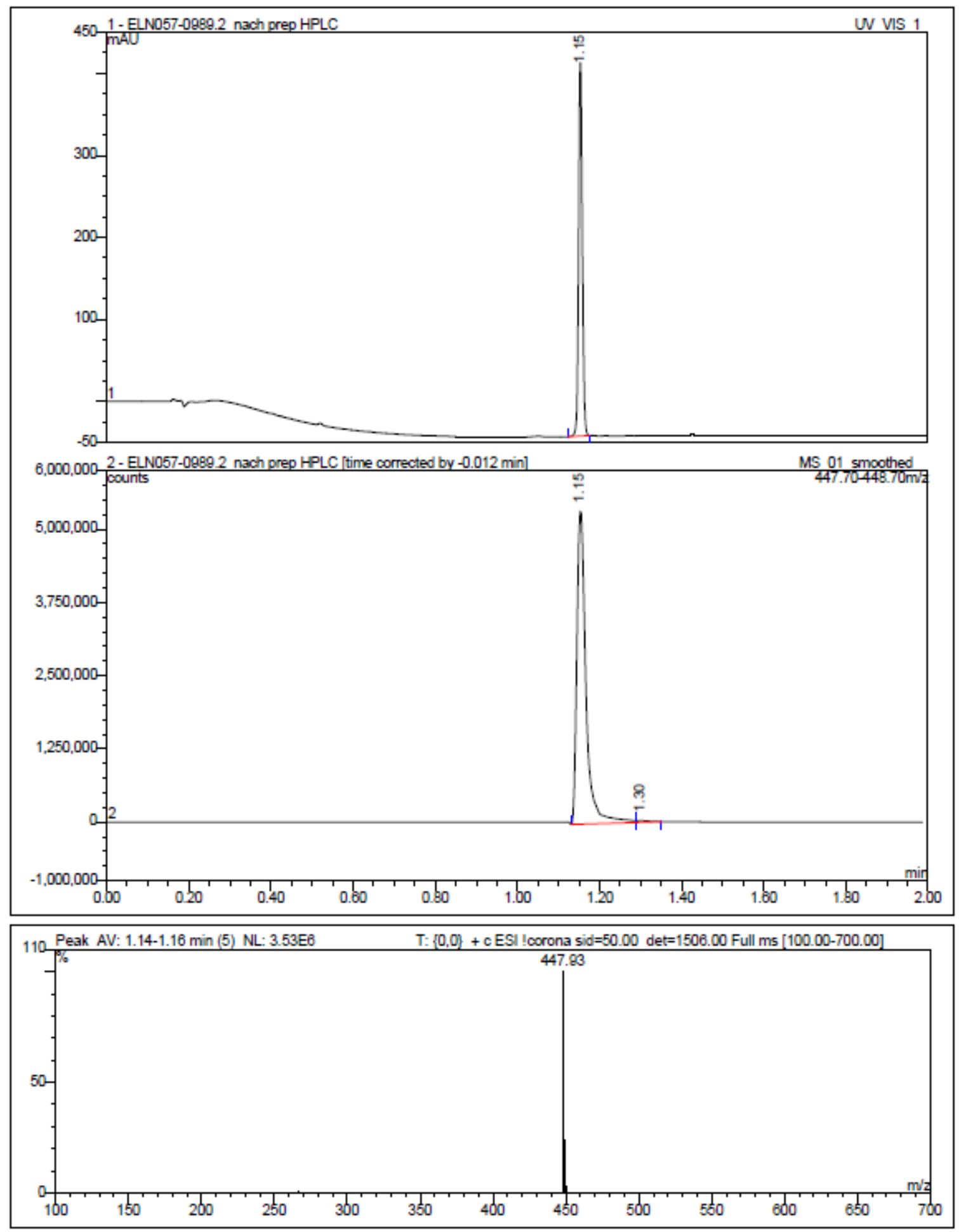


Compound 11h:
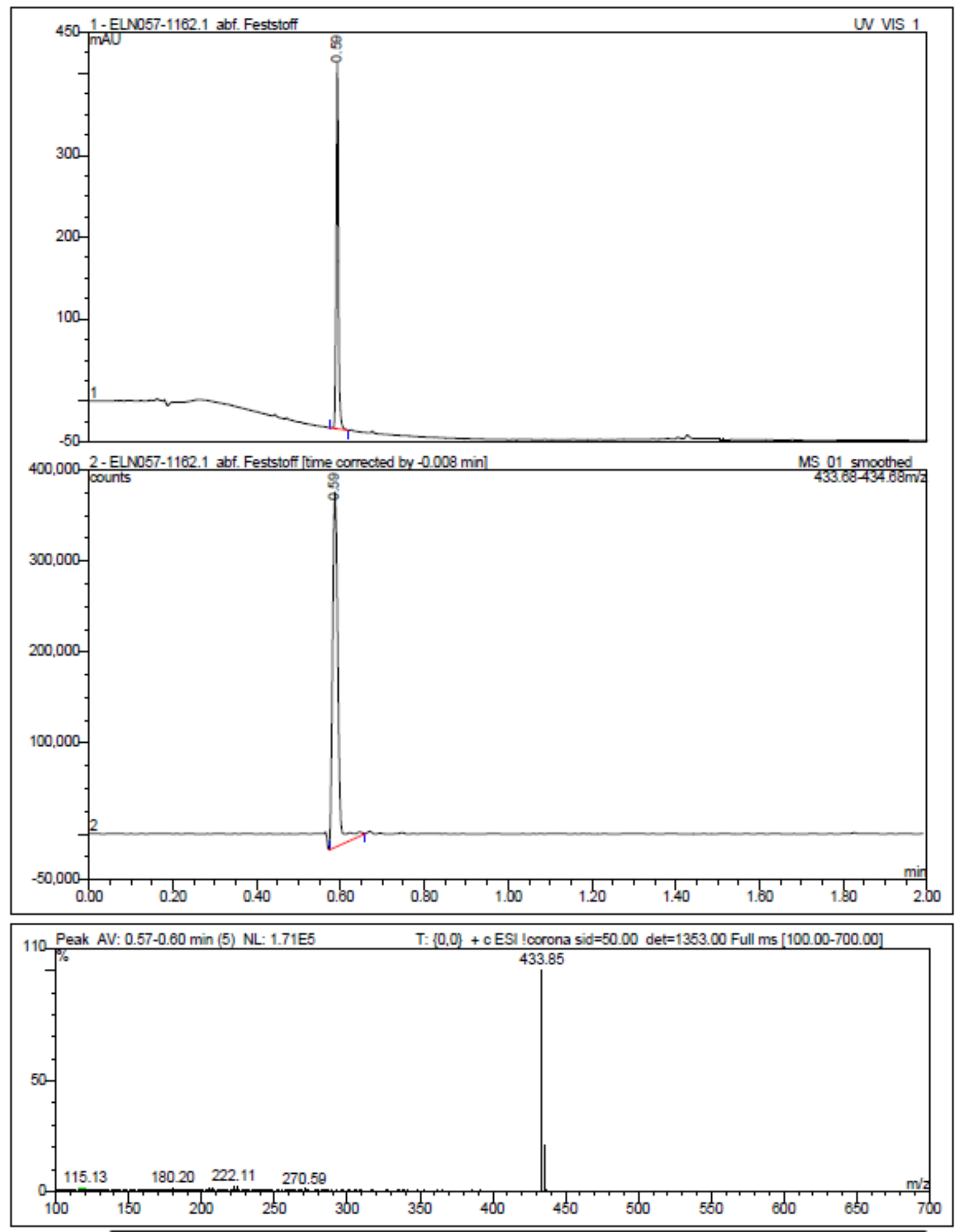
Compound 11i:
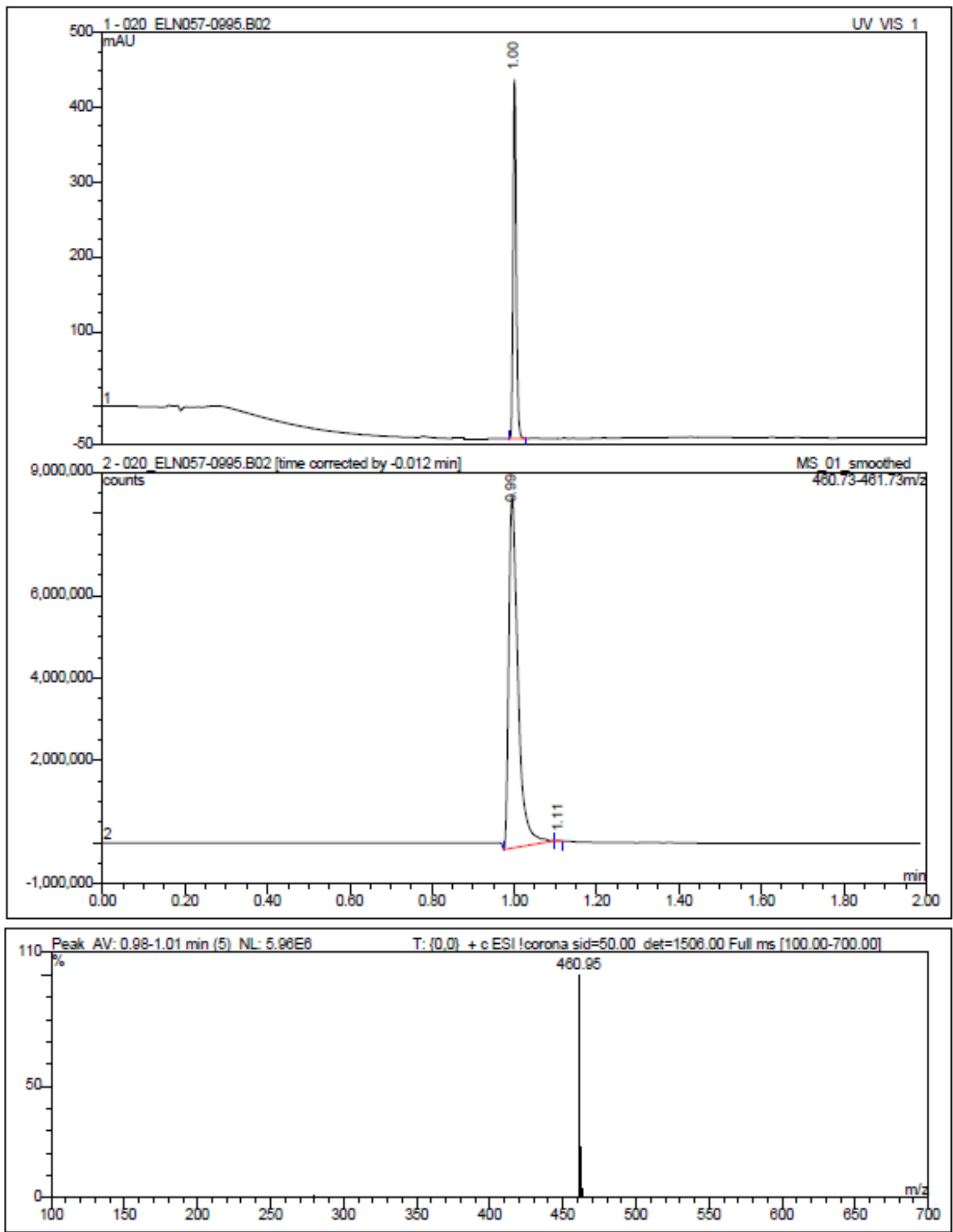
Compound $(\boldsymbol{R}, \boldsymbol{R})-\mathbf{1 1} \mathbf{i}$ :
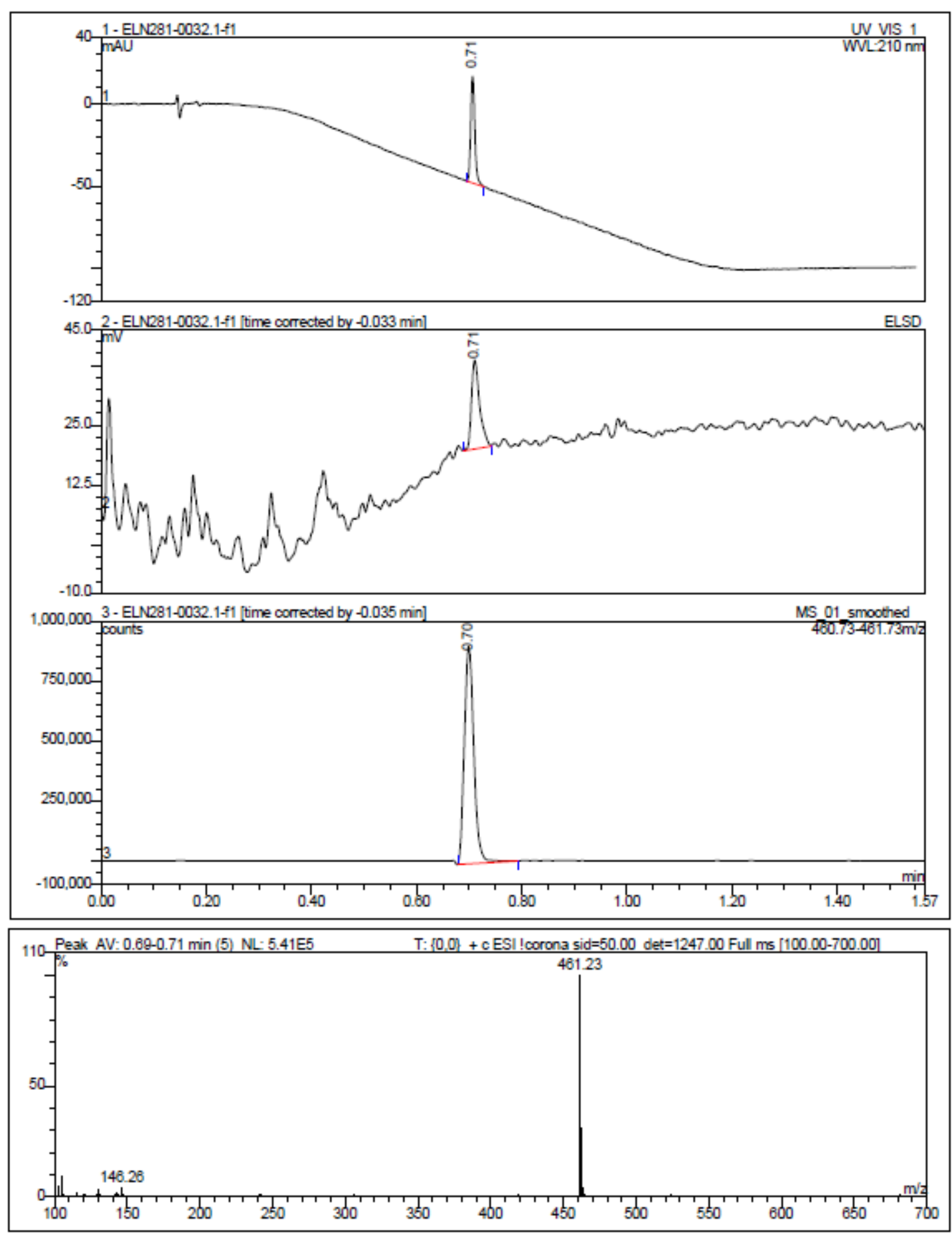


\begin{tabular}{lll|} 
Sample ID: & b446957a-20dd-11e5-ac35-001e2abd2a4c & Date: 02.07 .15 \\
Sample Name: & ELN281-0032.1 & \\
Sample Number: & 4 & \\
Time Base: & CHIRALLC04 \\
Datasource Name: & HPLC003_local \\
Sequence Name: & ELN281-0032.1 \\
Sequence Dir: & DatalCHIRALLC04lerharmi1ICXCR7 \\
Quantif. Method: & default \\
Injection Volume: & $5.00 \mathrm{ul}$ Comment: 1mg/ml Heptane/EtOH 1:1 \\
Eluent A: & $30.0 \%$ Heptane $0.05 \%$ DEA \\
Eluent $B:$ & $70.0 \%$ Ethanol $0.05 \%$ DEA \\
Flow: & $0.800 \mathrm{ml} / \mathrm{min}$ \\
Column: & (R,R) Whelk-01 250x4.6mm ID,5um \\
Serial number: & 100487 \\
Temperature: & $25.0{ }^{\circ} \mathrm{C}$ \\
Detection: & $210 \mathrm{~nm}$ \\
&
\end{tabular}

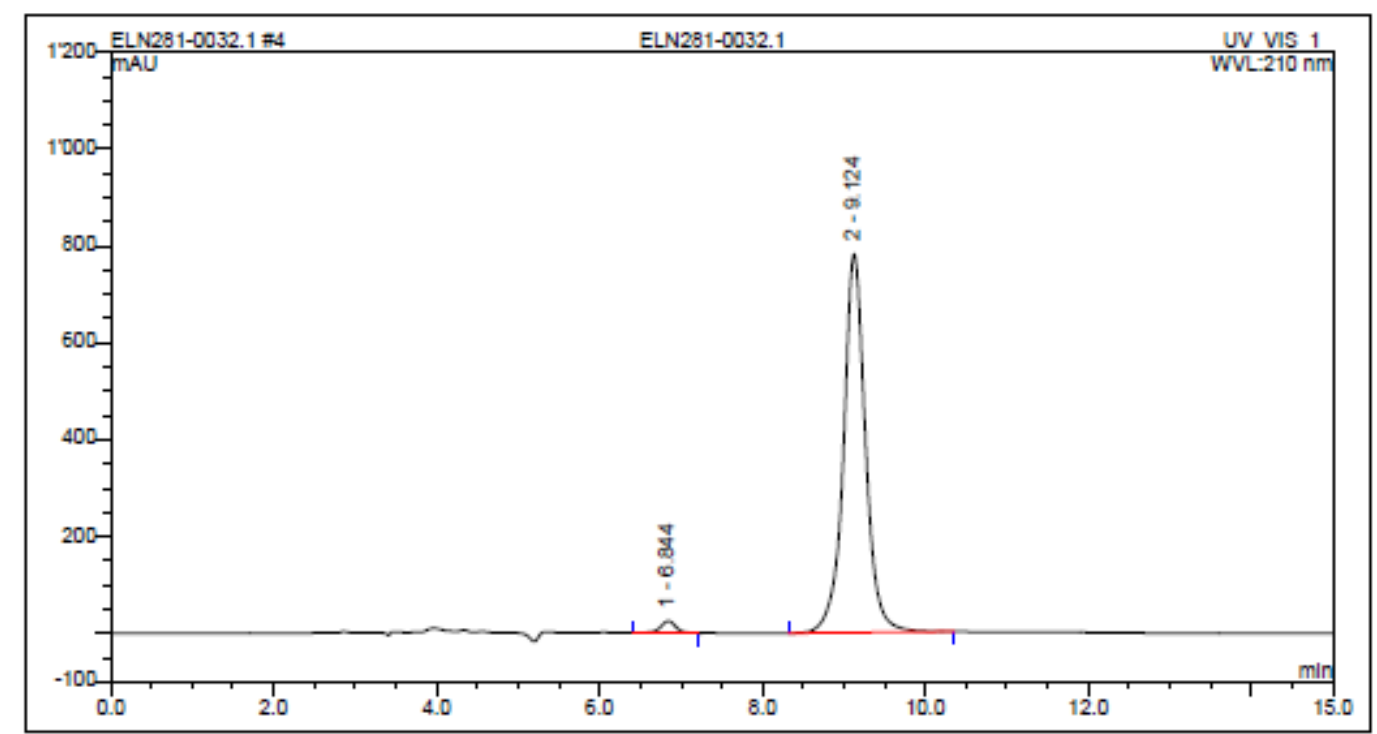

\begin{tabular}{cccccccc}
\hline $\begin{array}{c}\text { Peak No. } \\
\text { UV_VIS_1 }\end{array}$ & $\begin{array}{c}\text { Ret.Time } \\
\text { UV_VIS_1 } \\
\text { min }\end{array}$ & $\begin{array}{c}\text { Height } \\
\text { UV_VIS_1 } \\
\text { mAU }\end{array}$ & $\begin{array}{c}\text { Rel.Area } \\
\text { UV_VIS_1 } \\
\text { \% }\end{array}$ & $\begin{array}{c}\text { Area } \\
\text { UV_VIS_1 } \\
\text { mAU*min }\end{array}$ & $\begin{array}{c}\text { Resolution } \\
\text { UV_VIS_1 }\end{array}$ & $\begin{array}{c}\text { Asymmetry } \\
\text { UV_VIS_1 }\end{array}$ & $\begin{array}{c}\text { Plates } \\
\text { UV_VIS_1 }\end{array}$ \\
\hline 1 & 6.8 & 25 & 2.1 & 5.354 & 5.9 & 0.9 & 7251 \\
\hline & 9.1 & 781 & 97.9 & 244.917 & n.a. & 1.0 & 6397 \\
\hline Total: & & & 100.0 & 250.3 & & & \\
\hline
\end{tabular}


Compound $(\boldsymbol{S}, \boldsymbol{S})-\mathbf{1 1 i}$ :
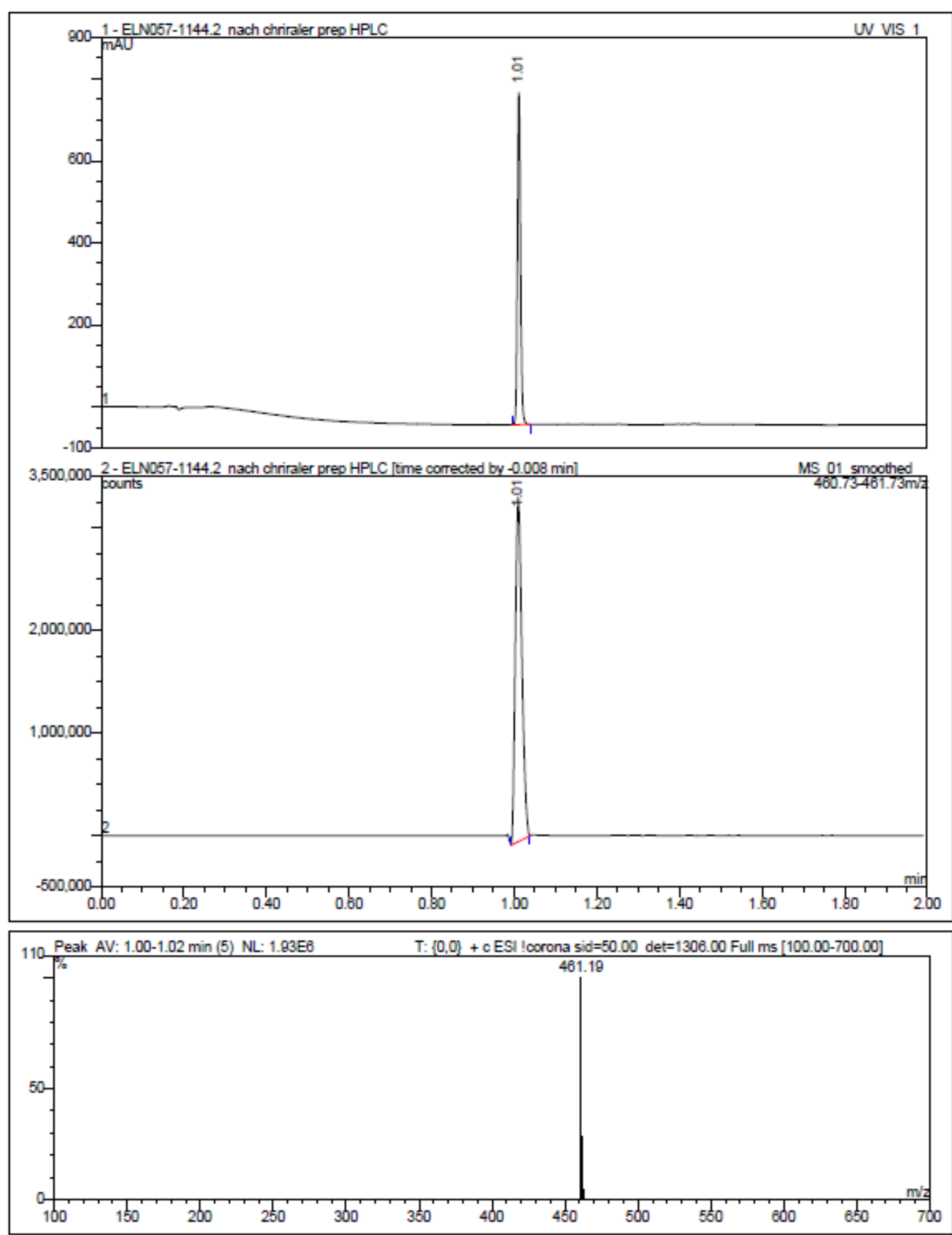
9c585f7c-3b68-11e5-ac35-001e2abd2a4c

Sample Name:

Sample Number:

Time Base:

Datasource Name:

Sequence Name:

Sequence Dir.

Quantif. Method:

Injection Volume:

Eluent A:

Eluent $B$ :

Flow:

Column:

Serial number:

Temperature:

ELN057-1144.2

Date: 05.08 .15

Detection:

CHIRALLC04

HPLC003_local

ELN057-1144.1

DataICHIRALLC04lerharmi1 ICXCR7

default

2.50 ul Comment: $1.2 \mathrm{mg} / \mathrm{ml}$ Heptane/EtOH 1:1

$30.0 \%$ Heptane $0.05 \%$ DEA

$70.0 \%$ Ethanol $0.05 \%$ DEA

$0.800 \mathrm{ml} / \mathrm{min}$

(R,R) Whelk-01 250x4.6mm ID,5um

100487

$25.0^{\circ} \mathrm{C}$

$210 \mathrm{~nm}$

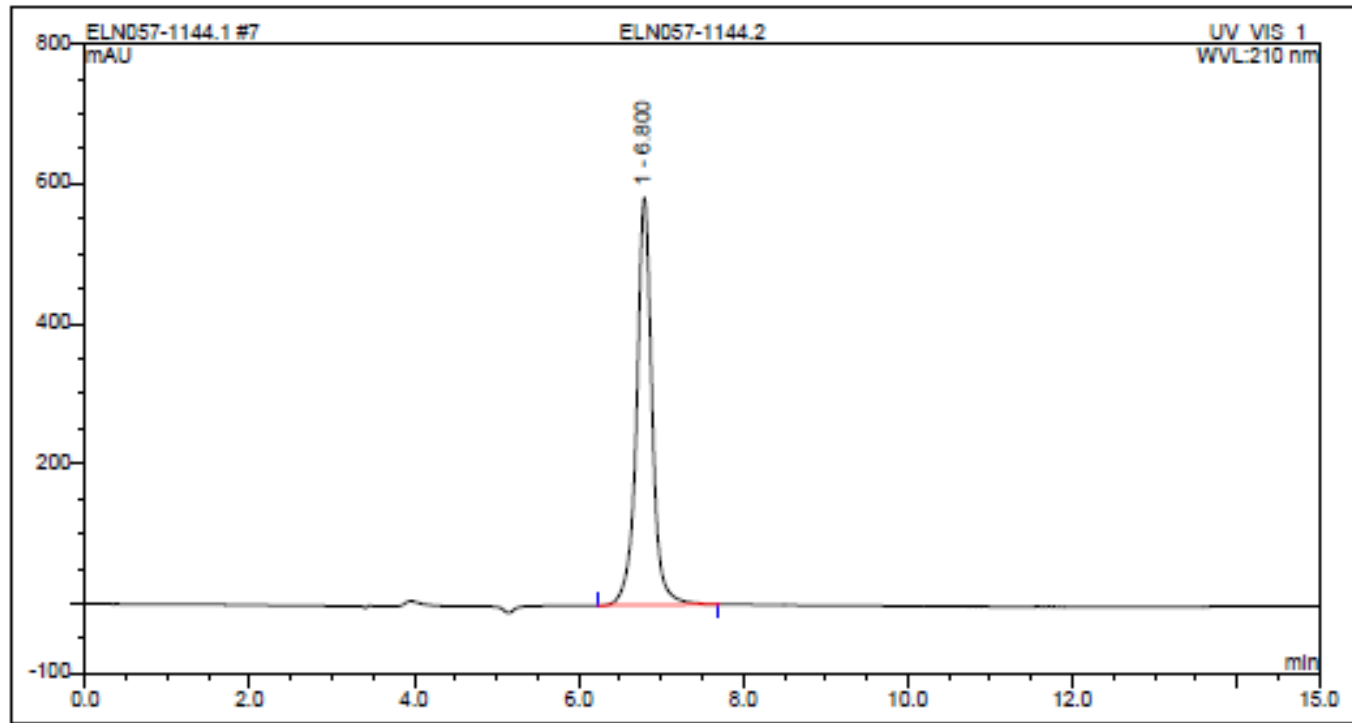

\begin{tabular}{lccccccc}
\hline $\begin{array}{c}\text { Peak No. } \\
\text { UV_VIS_1 }\end{array}$ & $\begin{array}{c}\text { Ret.Time } \\
\text { UV_VIS_1 } \\
\text { min }\end{array}$ & $\begin{array}{c}\text { Height } \\
\text { UV_VIS_1 } \\
\text { mAU }\end{array}$ & $\begin{array}{c}\text { Rel.Area } \\
\text { UV_VIS_1 } \\
\%\end{array}$ & $\begin{array}{c}\text { Area } \\
\text { UV_VIS_1 } \\
\text { mAU*min }\end{array}$ & $\begin{array}{c}\text { Resolution } \\
\text { UV_VIS_1 }\end{array}$ & $\begin{array}{c}\text { Asymmetry } \\
\text { UV_VIS_1 }\end{array}$ & $\begin{array}{c}\text { Plates } \\
\text { UV_VIS_1 }\end{array}$ \\
\hline Total: & 6.8 & 582 & 100.0 & 127.438 & n.a. & 1.0 & 7525 \\
\hline
\end{tabular}


Compound 11j:

Sample Name: LCMSP384-20200528-1_A05 ACT No: ACT-747599

Instrument: ICHALW-DL00021-SQD

\section{Expression}

Auto-Comments

Auto-Summary

Purity

Target Mass

Target RT

UV:212216]

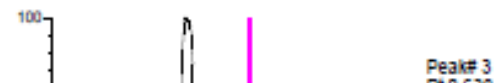

Peake 3
Rt $0.633^{2}$

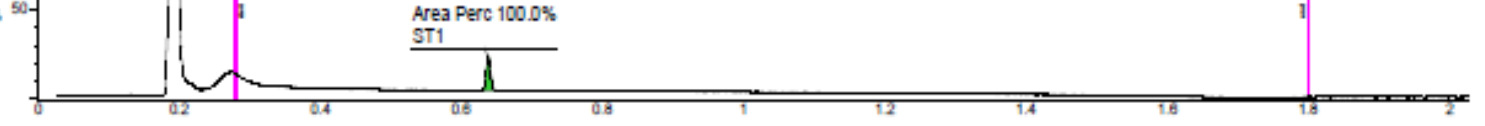

oid-6 4392051 (4528051-439.6051) w-0.5000

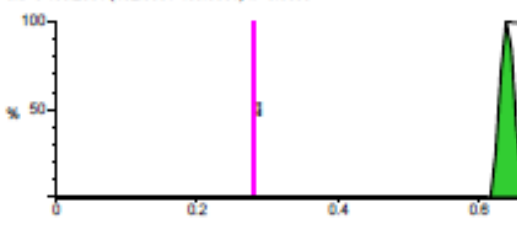

odd-11 431.18v5 (450 7aus-e31. 5805) w-0.8000

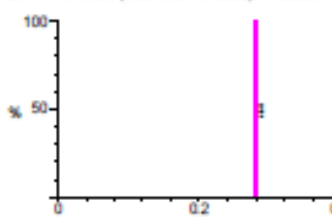

ELN Nr: ELN028-1219_A04

Acquisition time: 5/28/2020 9:18 AM Result

GOOD

100.0000

433.4000

0.6380
UV214 [SAEOB ]

Poak 2g0.343 min Ma(+) Es

$1.2 E 06$ onte
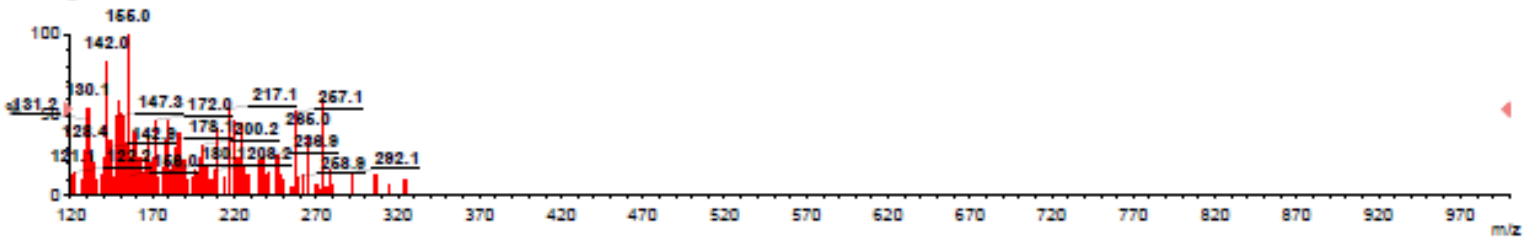

Poak 3go.ess min Mos(+) Es

2.7E07 onte

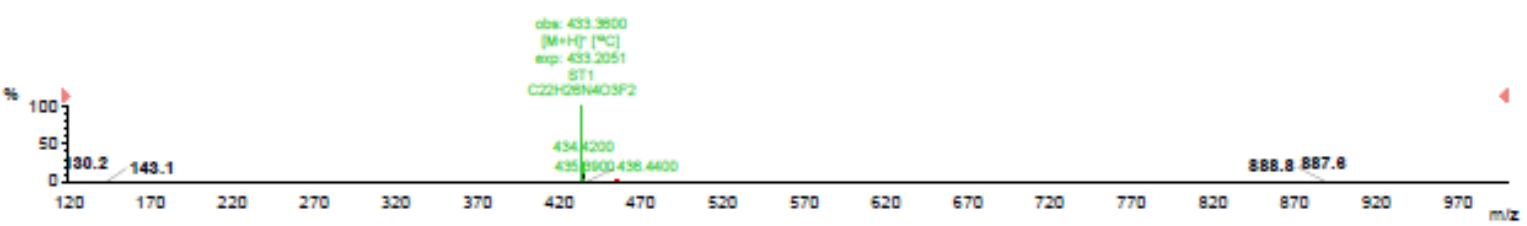


Compound 20a:
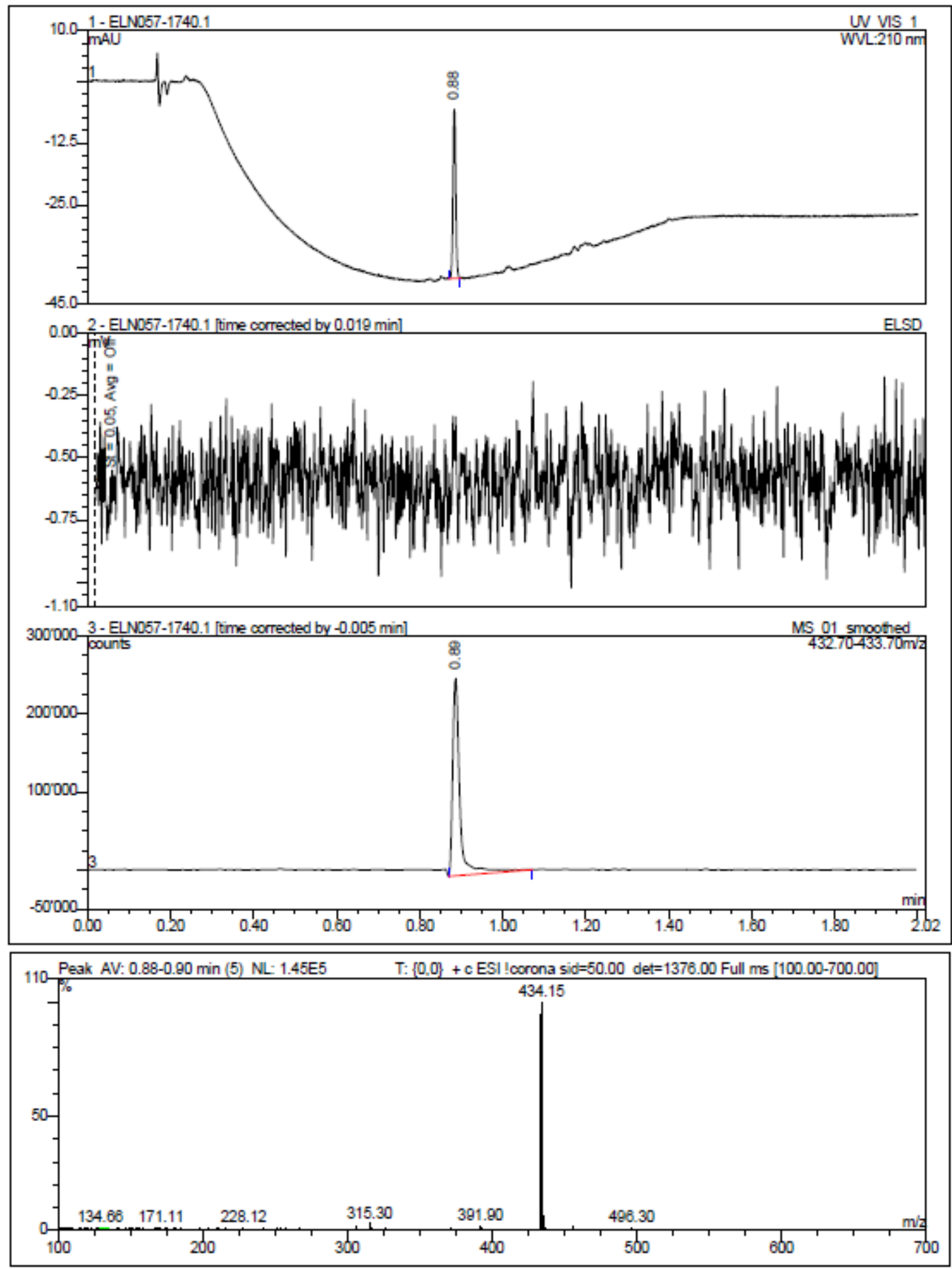
Compound 20b:
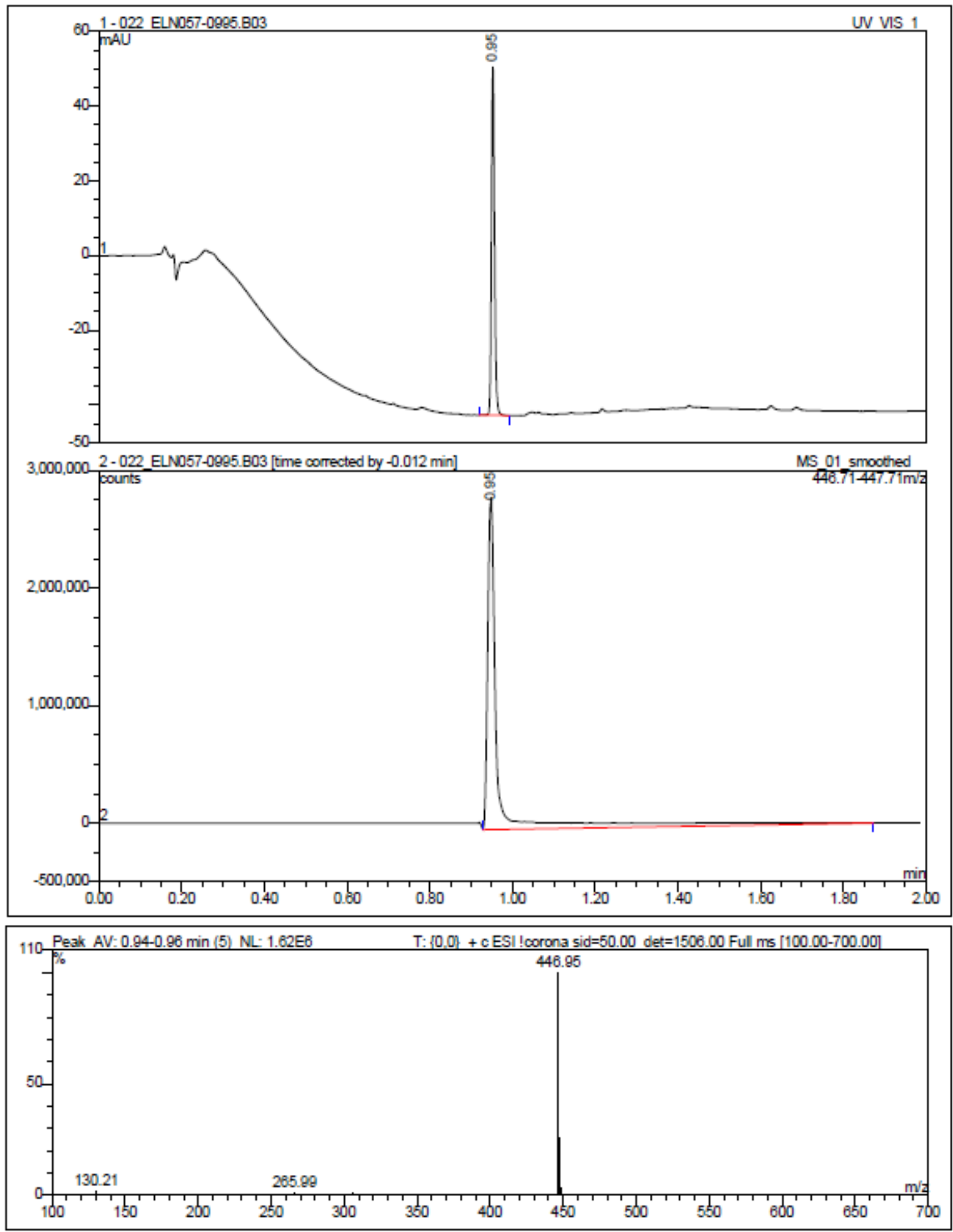
Compound 20c:
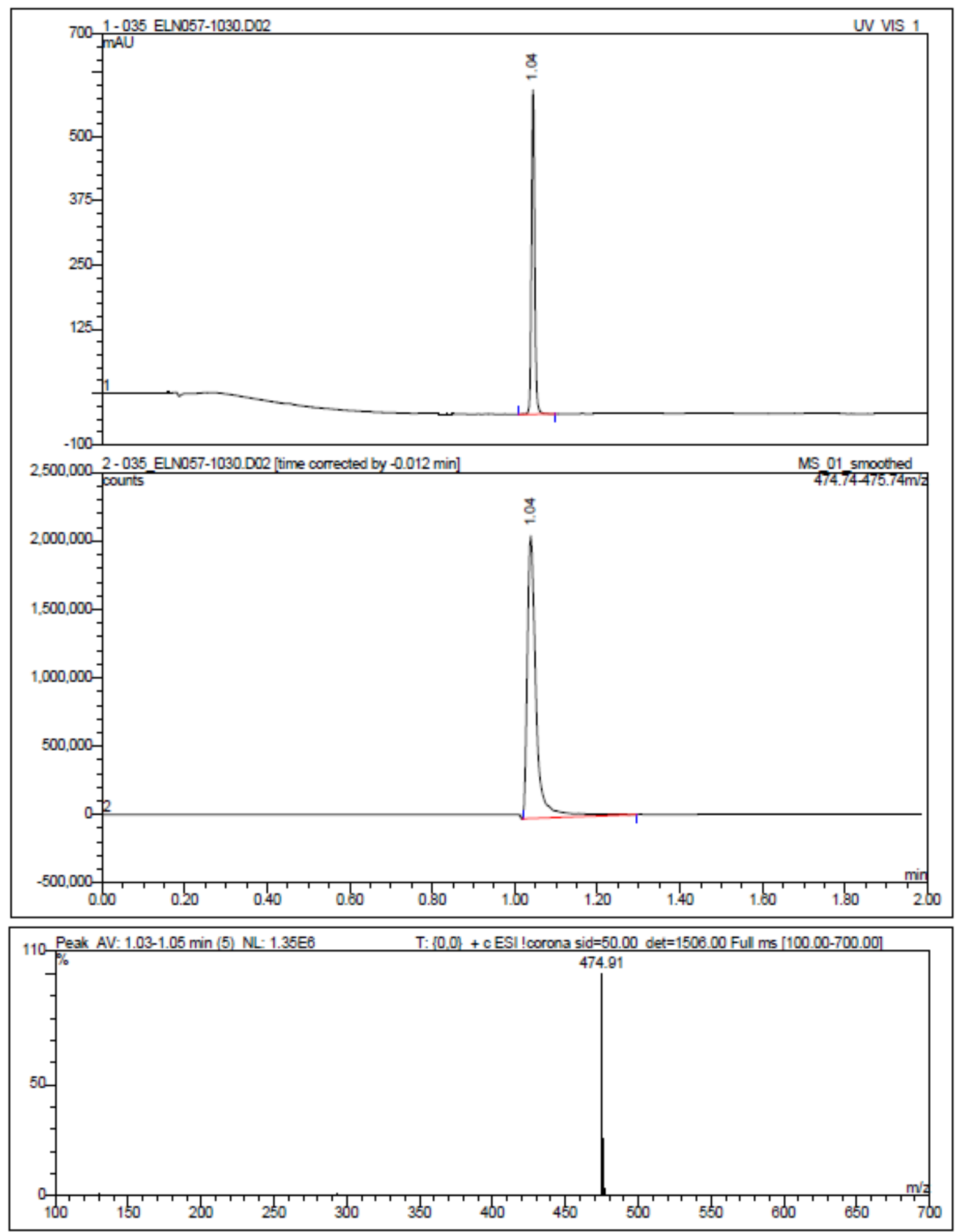
Compound 20d:
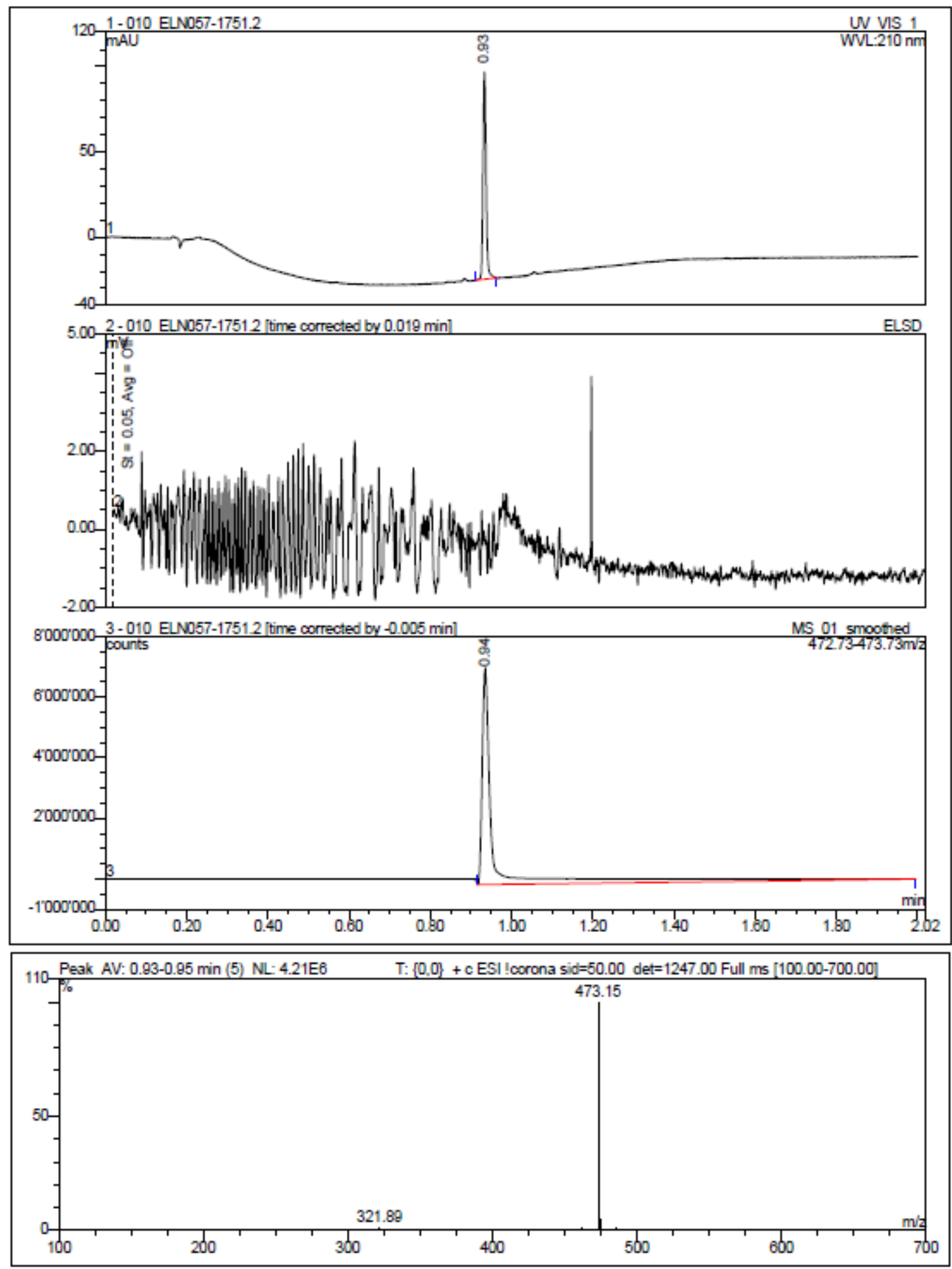
Compound 20e:
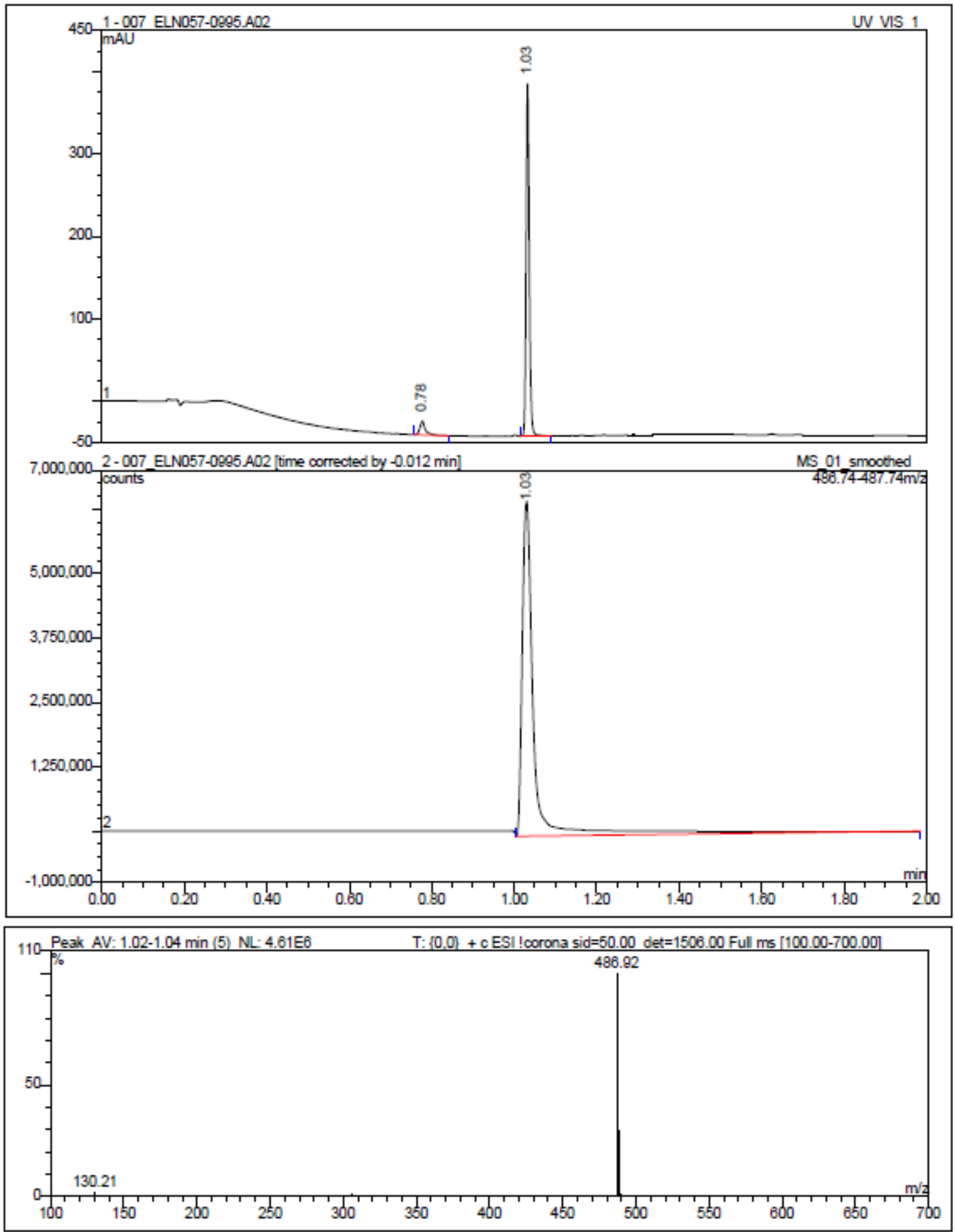
Compound 20f:
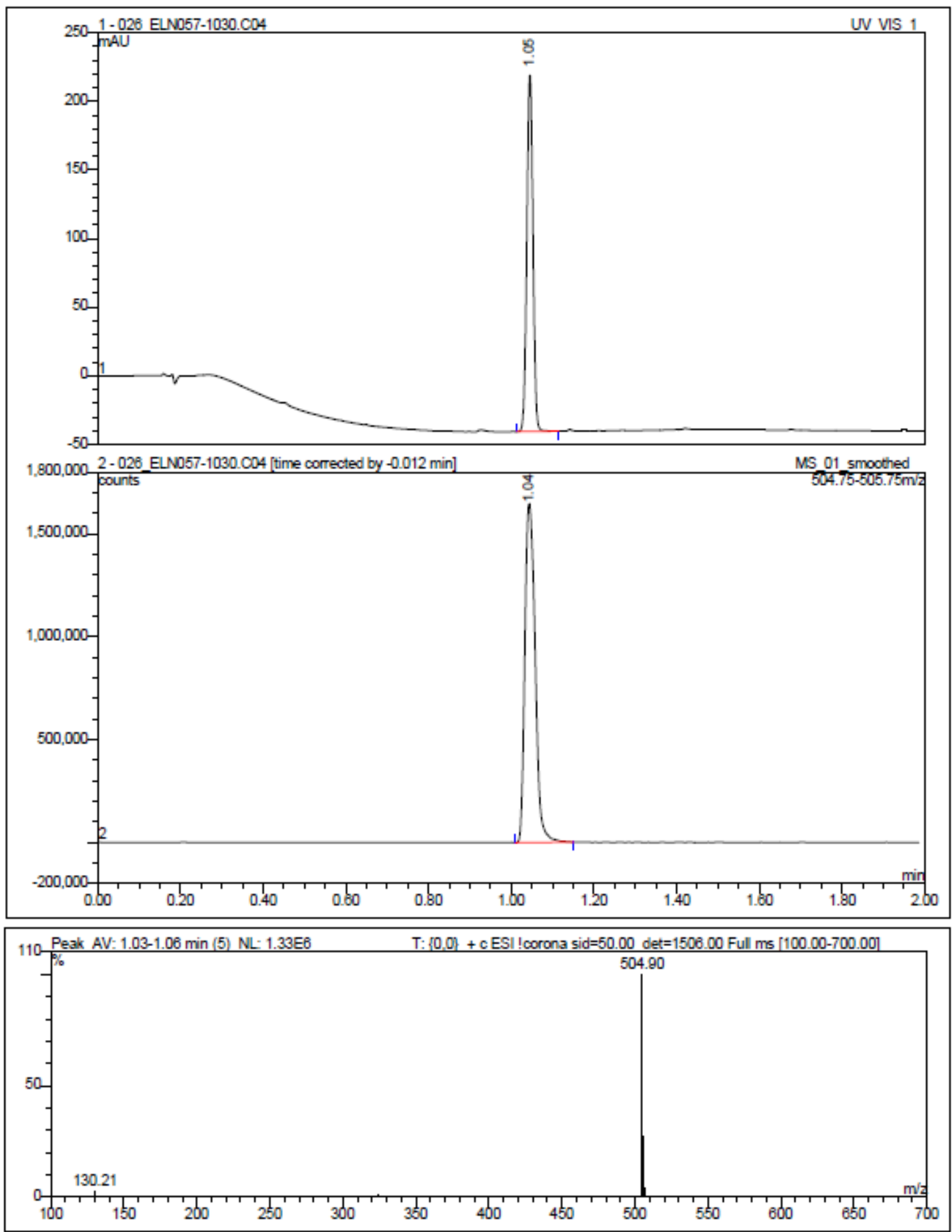
Compound 20g:
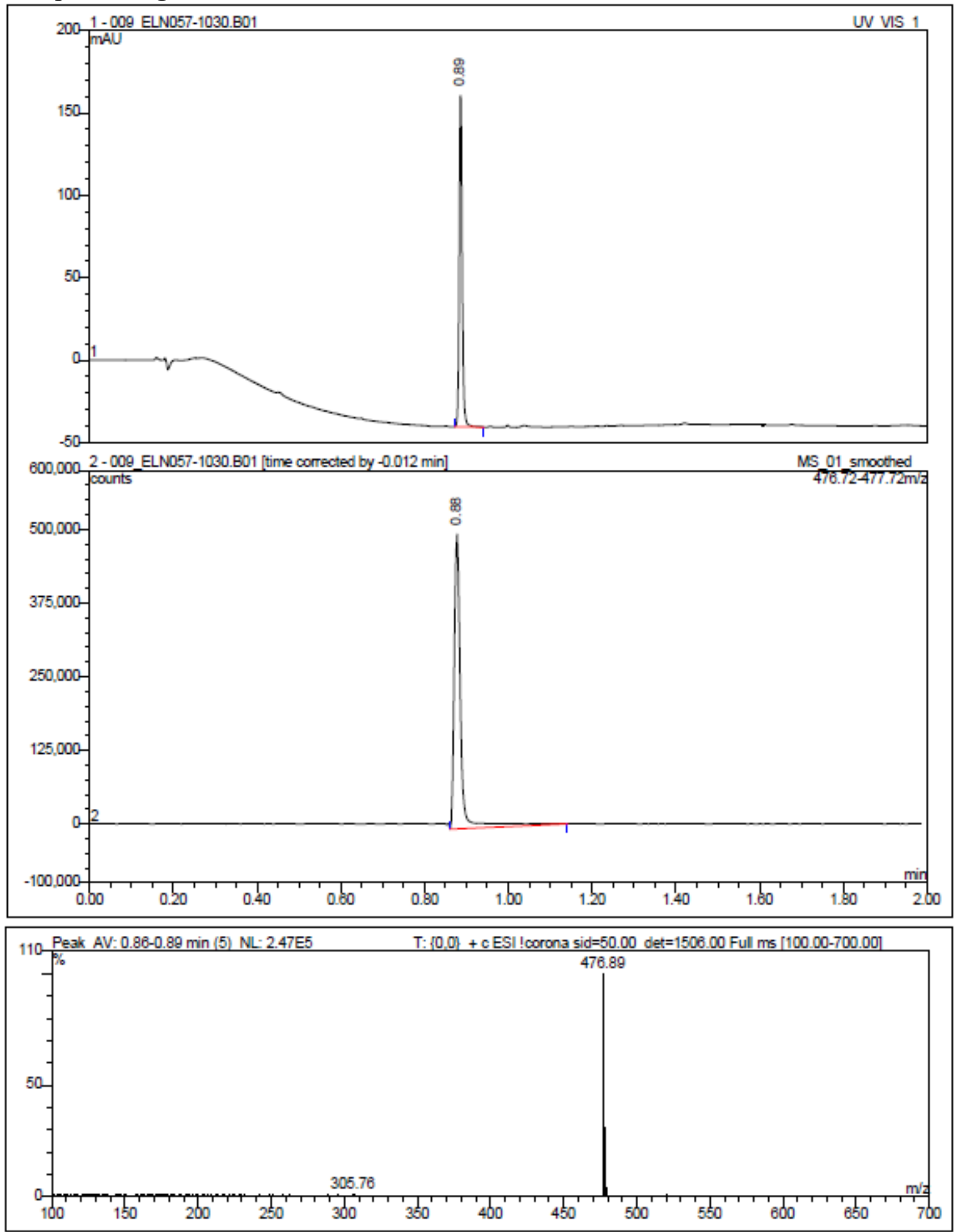
Compound 20h:
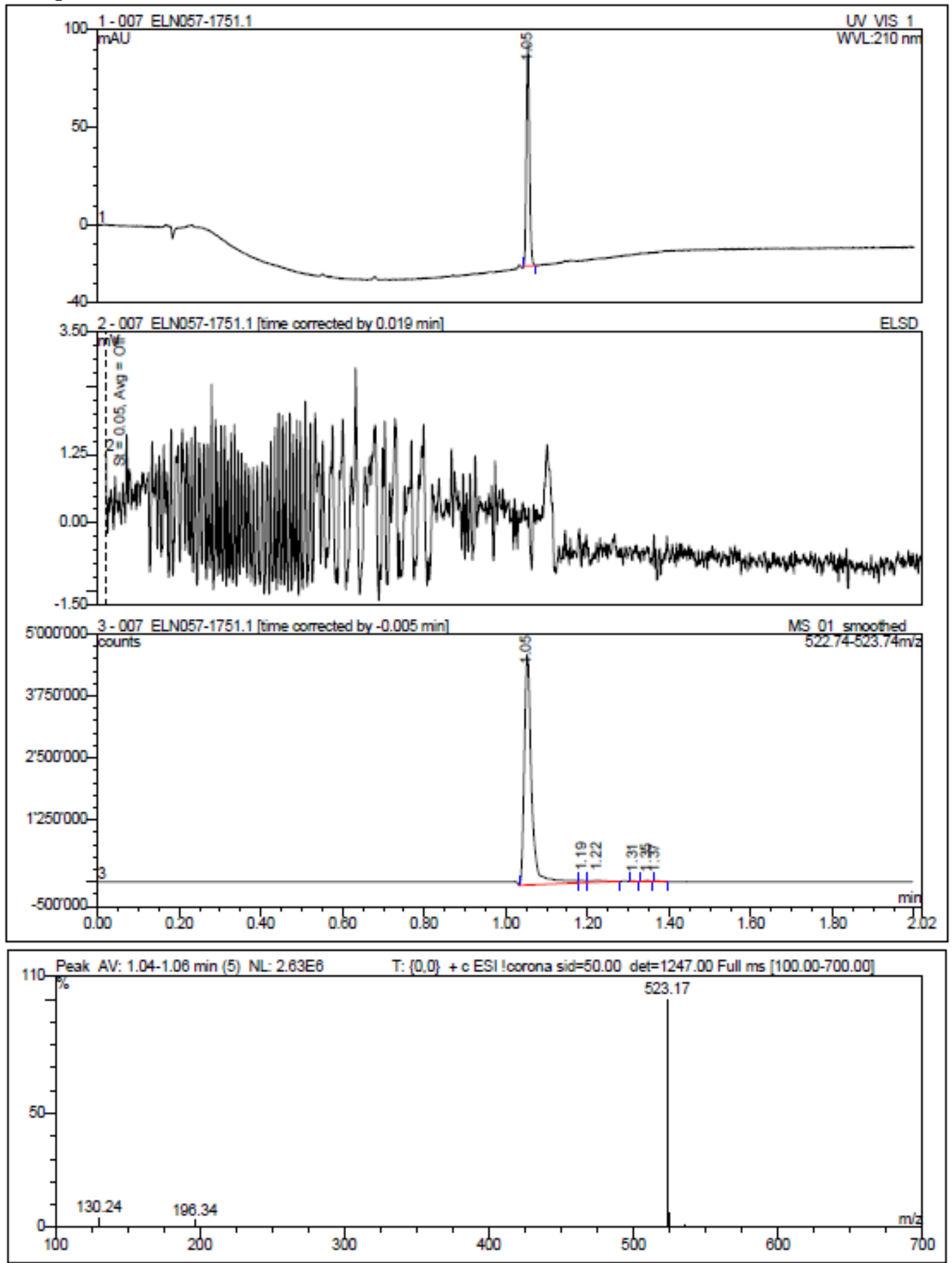
Compound 20i:

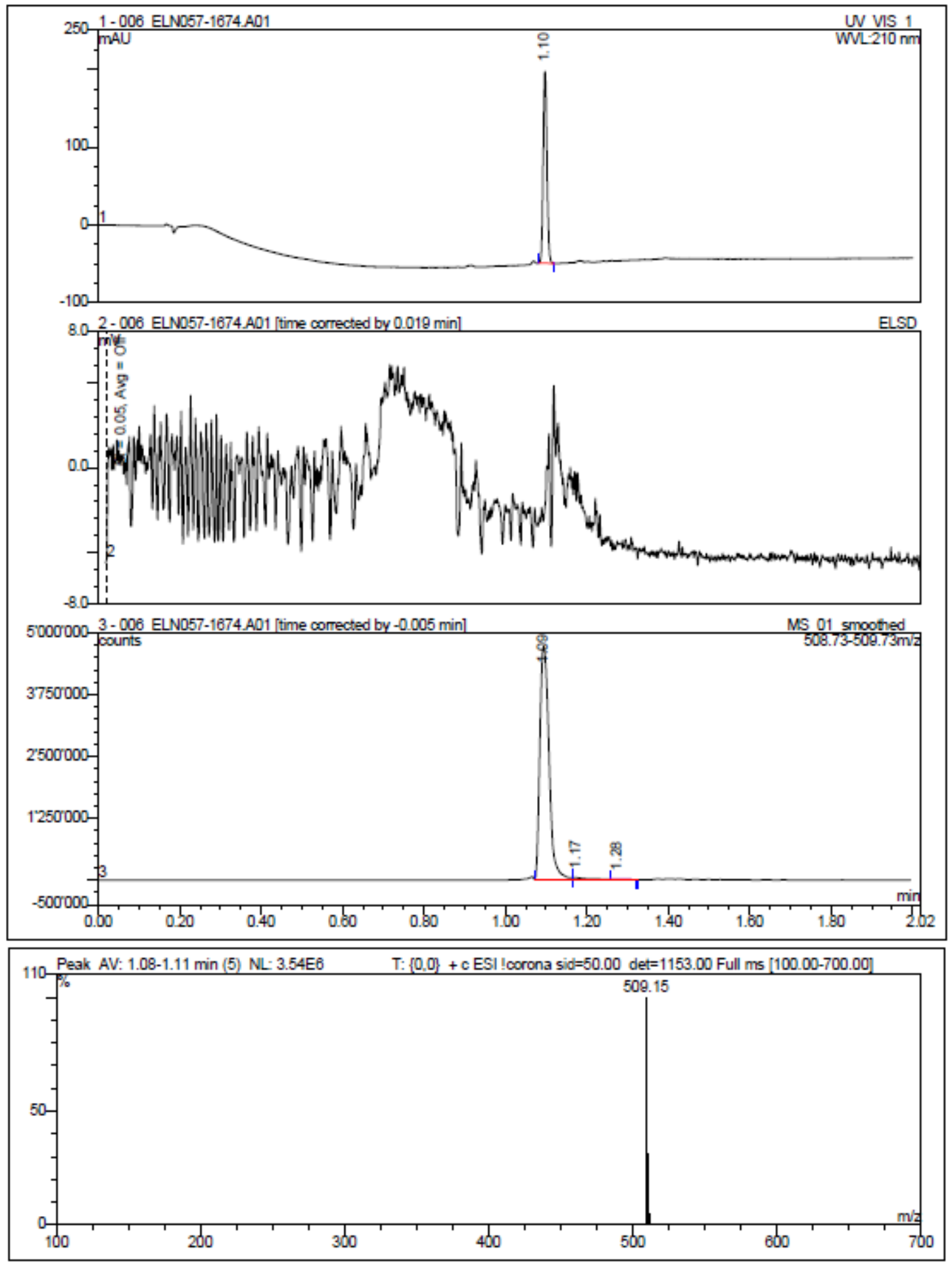


Compound 20j:
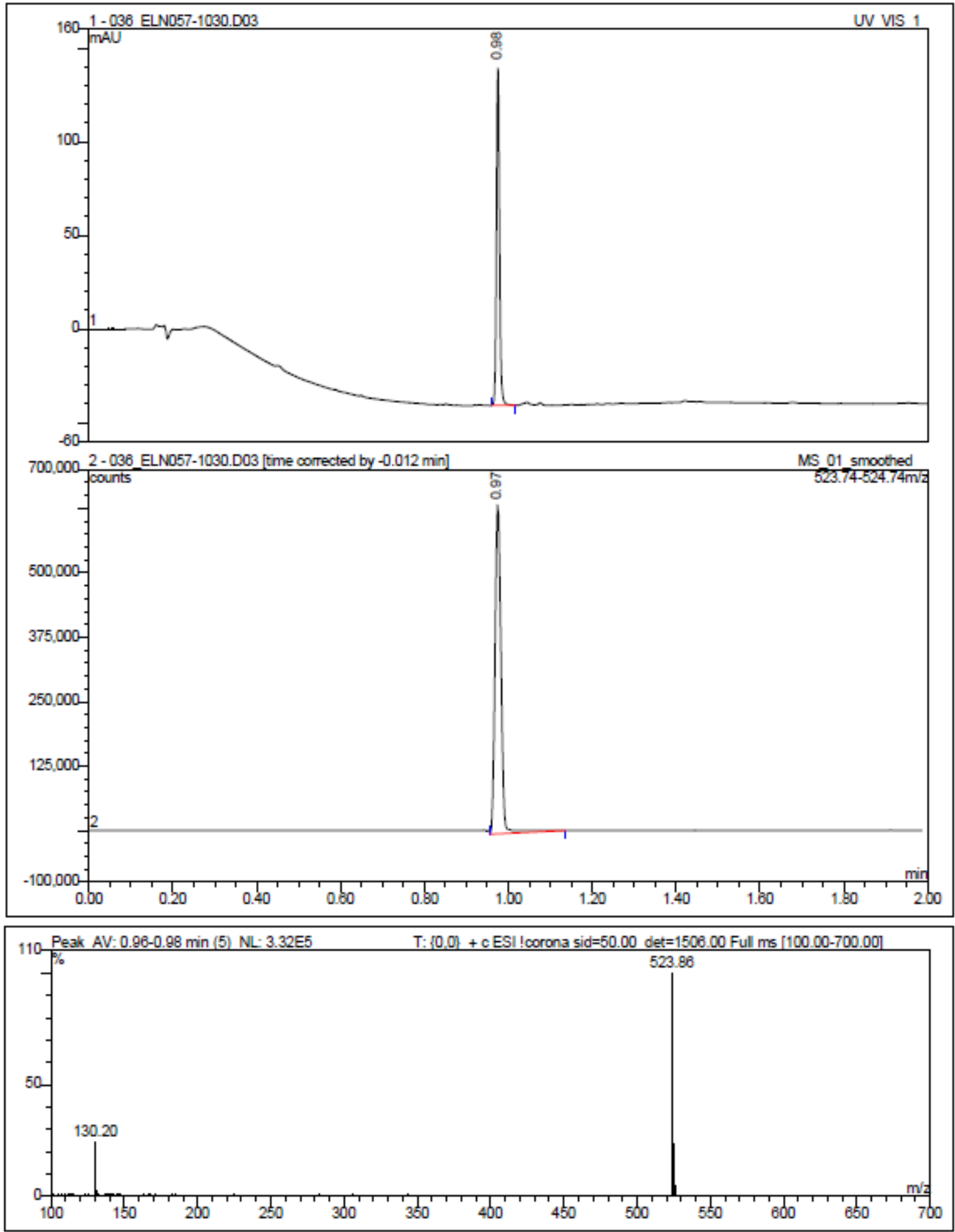
Compound 20k:
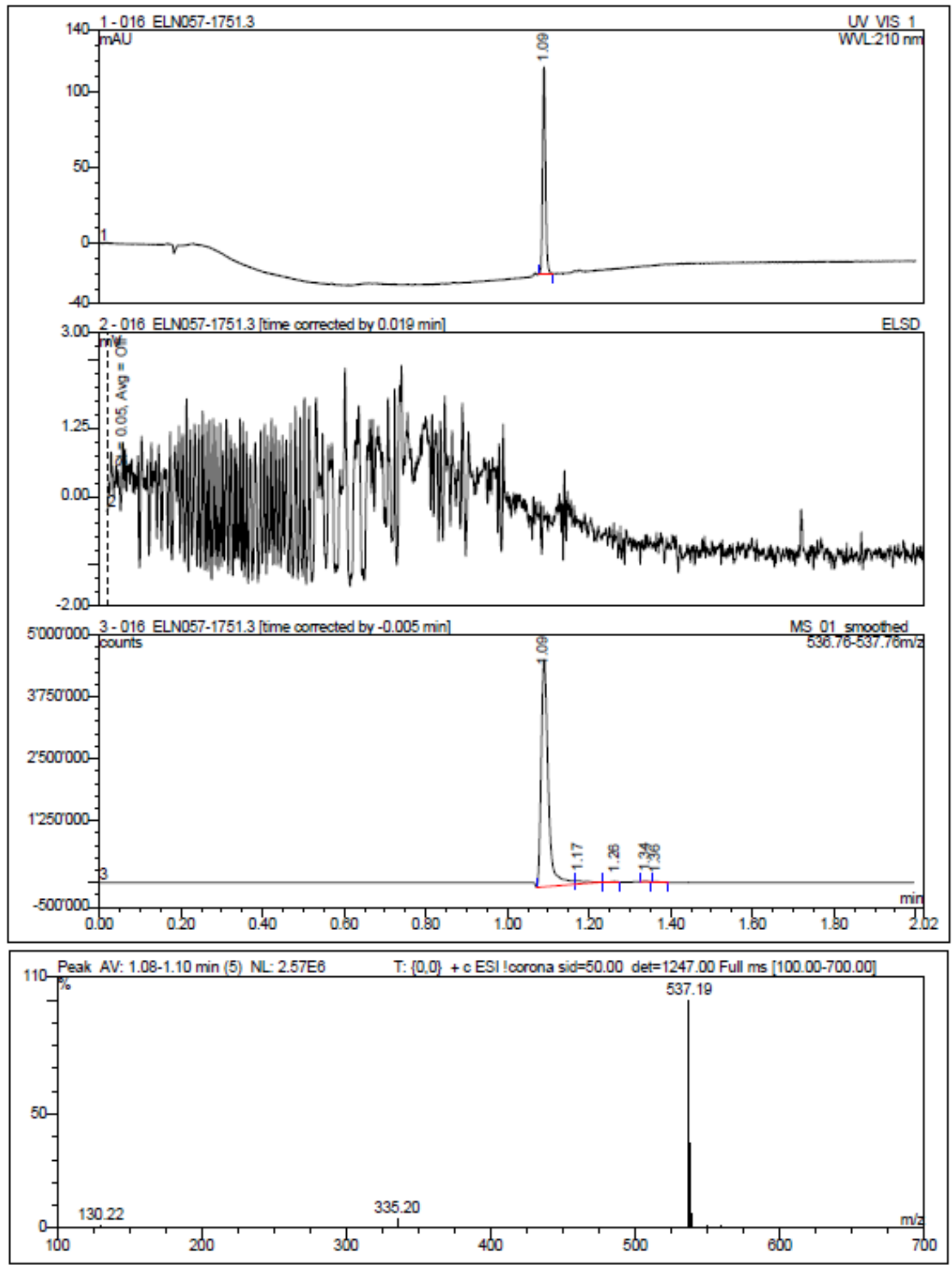
Compound 201:
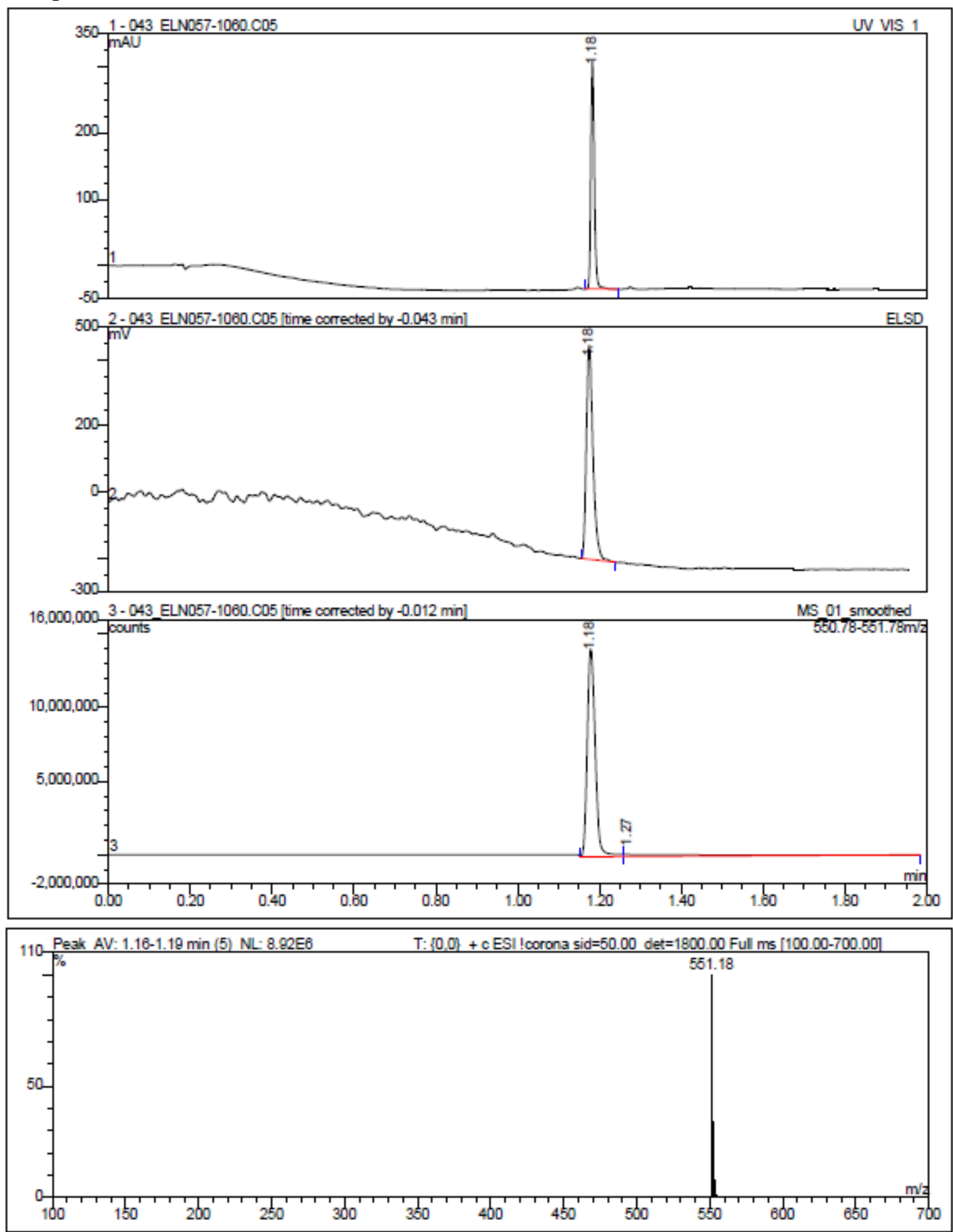
Compound 20m:

Sample Name: LCMS96-20150114-3_A03 ACT No: ACT-797084

Location: $4: 3$

Expression

Auto-Comments

Auto-Summary

Purity

Target Mass

4.UV[212.216]

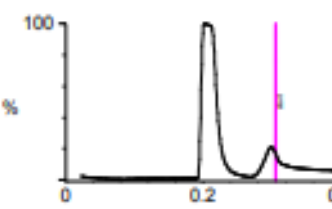

\section{Peaki=1
Rt 0.668}

Peakt 2

Area Perc $42.6 \%$ fit 0.684 .
Instrument: ACQ-SQD\#B09SQD437W

5. (1) ELso Signal

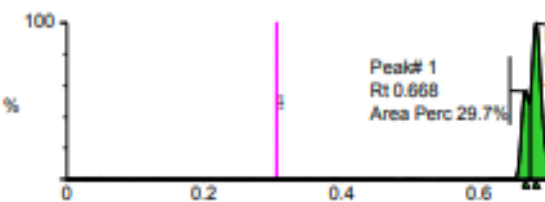

10. $[M+H] 538.2624(537.7624 .638 .7624) w=1.0000$

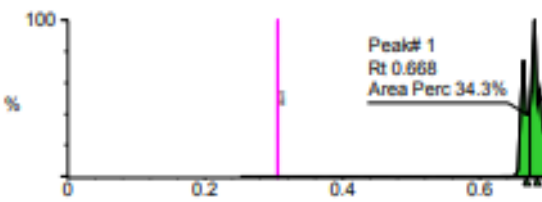

UV214

$\mathrm{T1}-\mathrm{ECC}(+)[\mathrm{M}+\mathrm{H}$
ELN Nr: ELN057-1060_A12

Acquisition time: 1/14/2015 6:44 PM

Result

Possible lsomers Present

GOOD

99.3000

269.4000

4038144.0

11- [M-H] 536.2479 (535.7479-536.7479) $w=1.0000$

$\min (4) 07$

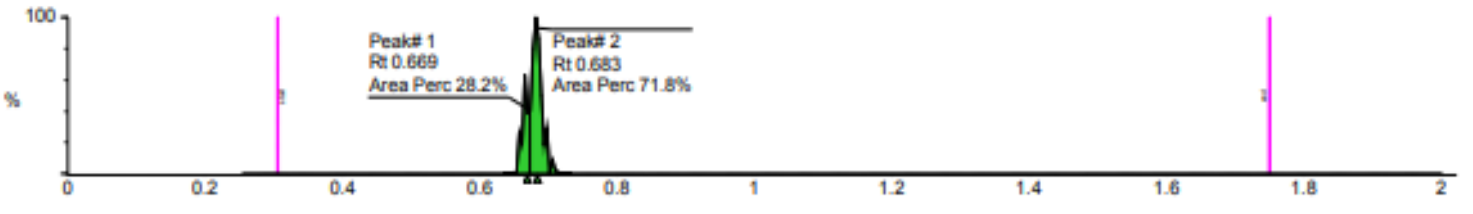

$\min$

Poak 1 ge. 668 min MS(+) ES

6.7E07 cnts

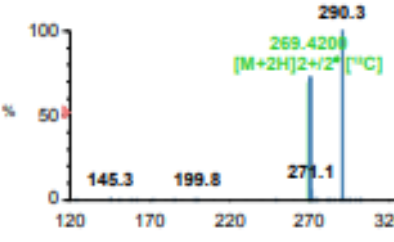

$\min _{305923.1}$

Poak 2 g0.679 min MS(+) ES

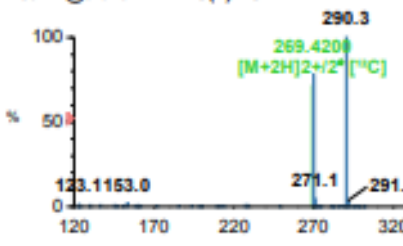

539 hens 560.3100 
Compound 20n:
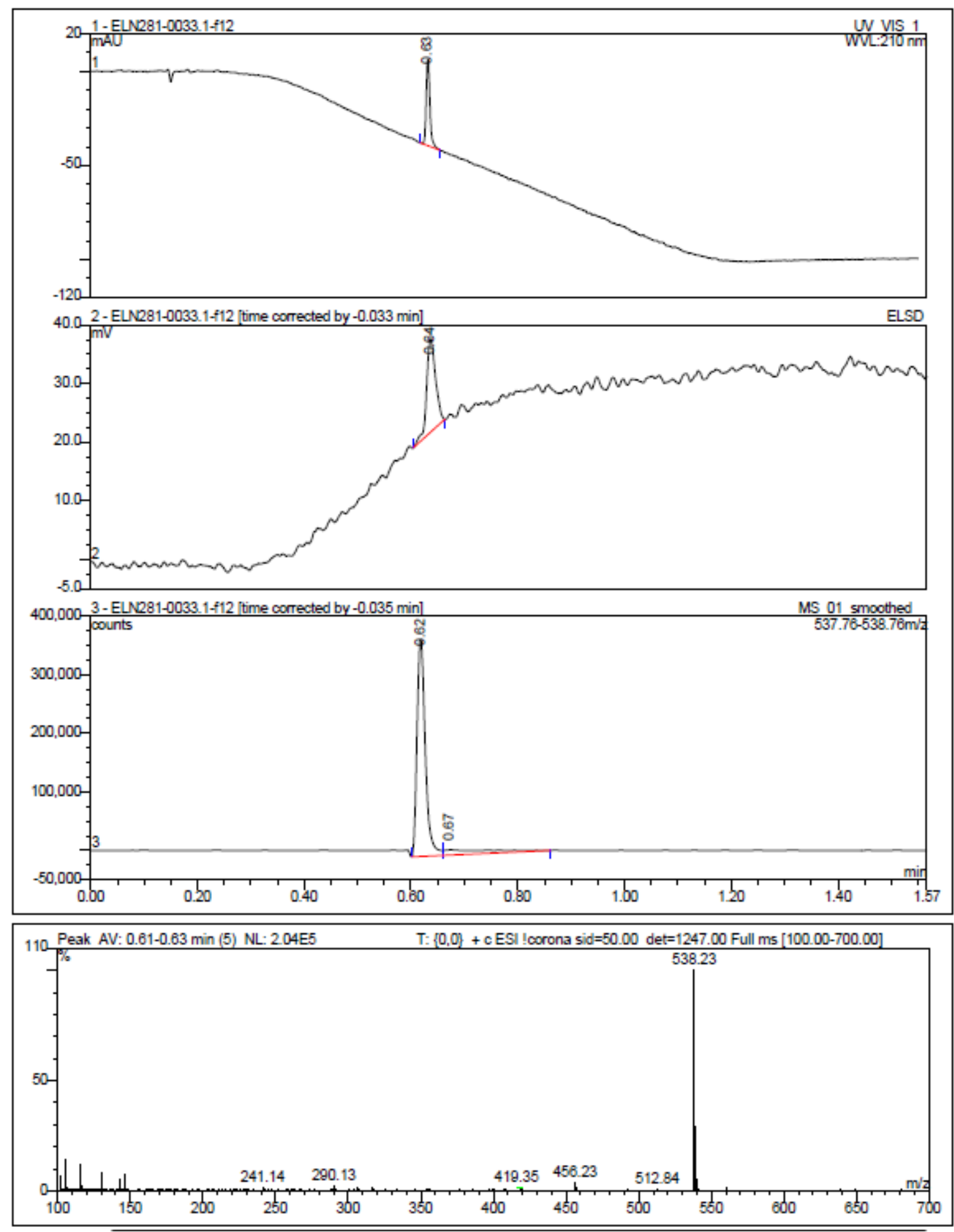


\begin{tabular}{|c|c|c|}
\hline Sample ID: & 322471f3-20d3-11e5-ba0c-0022191a3ba4 & \\
\hline Sample Name: & ELN281-0033.1 & Date: 02.07 .15 \\
\hline Sample Number: & 4 & \\
\hline Time Base: & U3000 & \\
\hline Datasource Name: & LCMSCHEM03_local & \\
\hline Sequence Name: & ELN281-0033.1 & \\
\hline Sequence Dir: & Datalerharmi1iSamplesiCXCR7 & \\
\hline Quantif. Method: & default & \\
\hline Injection Volume: & $4.00 \mathrm{ul}$ Comment: $1 \mathrm{mg} / \mathrm{mL}$ Heptan/Ethanol 1:1 & \\
\hline Eluent A: & $90.0 \%$ Heptane $0.05 \%$ DEA & \\
\hline Eluent $B$ : & $10.0 \%$ Ethanol $0.05 \%$ DEA & \\
\hline Flow: & $0.800 \mathrm{ml} / \mathrm{min}$ & \\
\hline Column: & Chiralpak IB 250x4.6mm ID, 5um & \\
\hline Serial number: & IB00CE-LI036 & \\
\hline Temperature: & $25.0^{\circ} \mathrm{C}$ & \\
\hline Detection: & $210 \mathrm{~nm}$ & \\
\hline
\end{tabular}

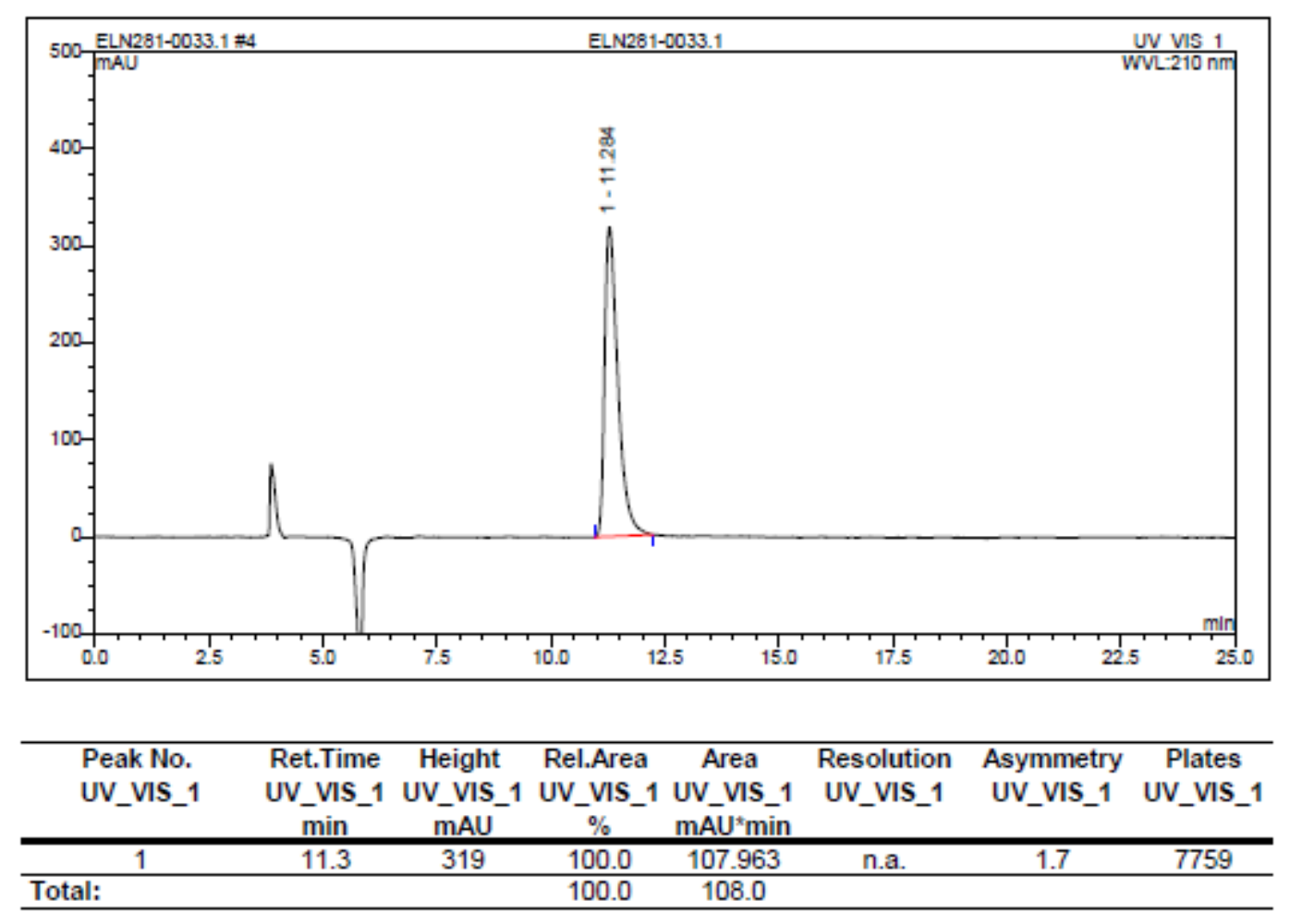


Compound 20o:
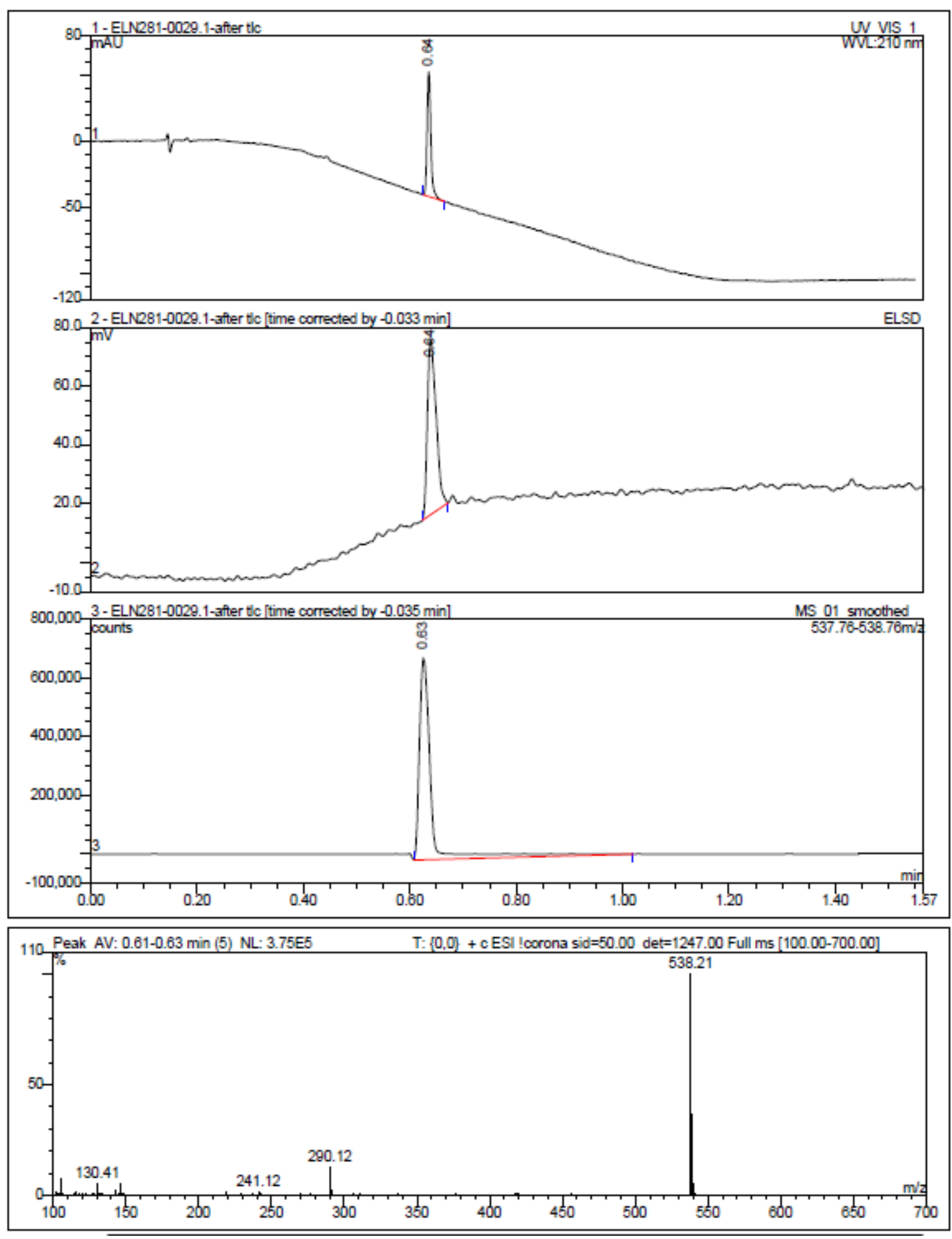
b0cd9997-1981-11e5-ba0c-0022191a3ba4

Sample Name:

Sample Number:

Time Base:

Datasource Name:

Sequence Name:

Sequence Dir.

Quantif. Method:

Injection Volume:

Eluent A:

Eluent $B$ :

Flow:

Column:

Serial number:

Temperature:

ELN281-0029.1

U3000

LCMSCHEM03_local

ELN281-0029.1

Datalerharmi1iSamplesiCXCR7

default

$5.00 \mathrm{ul}$ Comment: $1 \mathrm{mg} / \mathrm{mL}$ Heptan/Ethanol 3:1

$90.0 \%$ Heptane $0.05 \%$ DEA

$10.0 \%$ Ethanol $0.05 \%$ DEA

$0.800 \mathrm{ml} / \mathrm{min}$

Chiralpak IB 250x4.6mm ID, 5 um

IB00CE-LI036

$25.0^{\circ} \mathrm{C}$

Detection:

$210 \mathrm{~nm}$

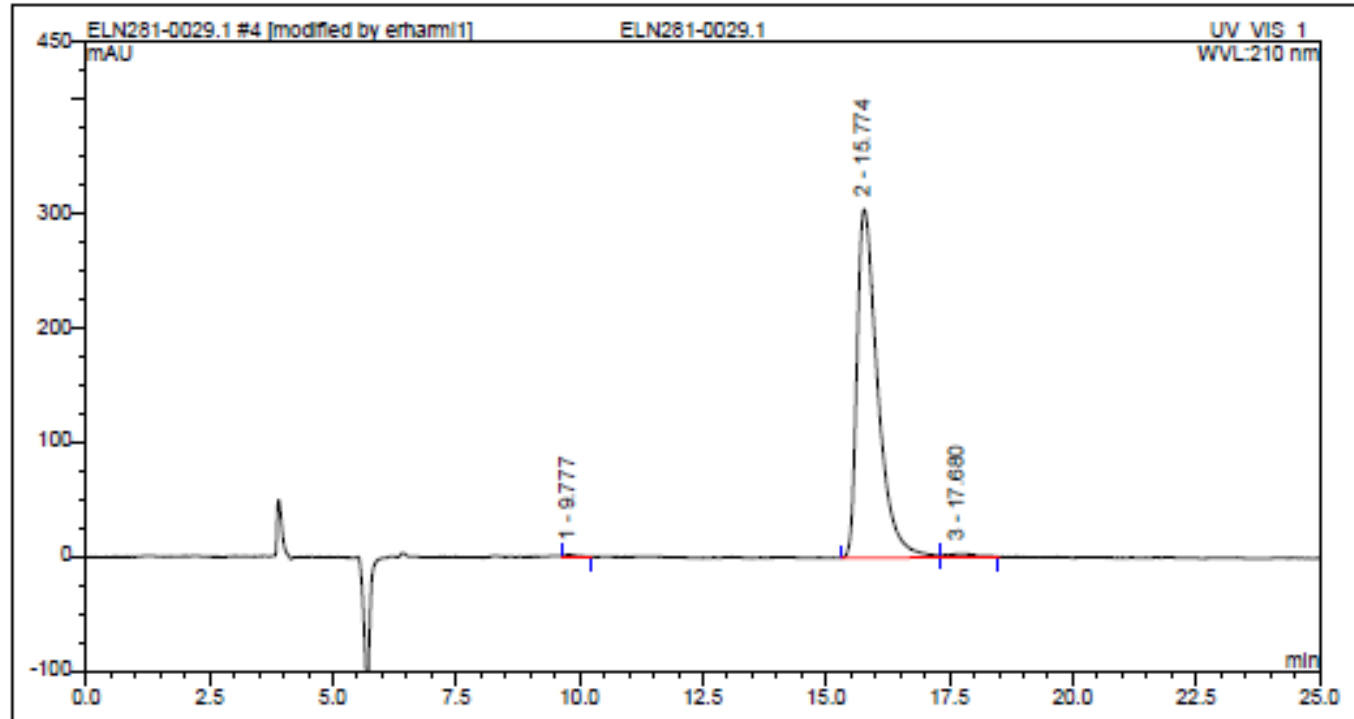

\begin{tabular}{|c|c|c|c|c|c|c|c|}
\hline $\begin{array}{l}\text { Peak No. } \\
\text { UV_VIS_1 }\end{array}$ & $\begin{array}{c}\text { Ret.Time } \\
\text { UV_VIS_1 } \\
\text { min }\end{array}$ & $\begin{array}{c}\text { Height } \\
\text { UV_VIS_1 } \\
\text { mAU }\end{array}$ & $\begin{array}{c}\text { Rel.Area } \\
\text { UV_VIS_1 } \\
\%\end{array}$ & $\begin{array}{c}\text { Area } \\
\text { UV_VIS_1 } \\
\text { mAU*min }\end{array}$ & $\begin{array}{l}\text { Resolution } \\
\text { UV_VIS_1 }\end{array}$ & $\begin{array}{l}\text { Asymmetry } \\
\text { UV_VIS_1 }\end{array}$ & $\begin{array}{c}\text { Plates } \\
\text { UV_VIS_1 }\end{array}$ \\
\hline 1 & 9.8 & 2 & 0.4 & 0.612 & 10.2 & n.a. & 7675 \\
\hline 2 & 15.8 & 304 & 98.2 & 148.244 & n.a. & 1.8 & 7460 \\
\hline 3 & 17.7 & 3 & 1.4 & 2.167 & n.a. & n.a. & n.a. \\
\hline Total: & & & 100.0 & 151.0 & & & \\
\hline
\end{tabular}


Compound 20p:
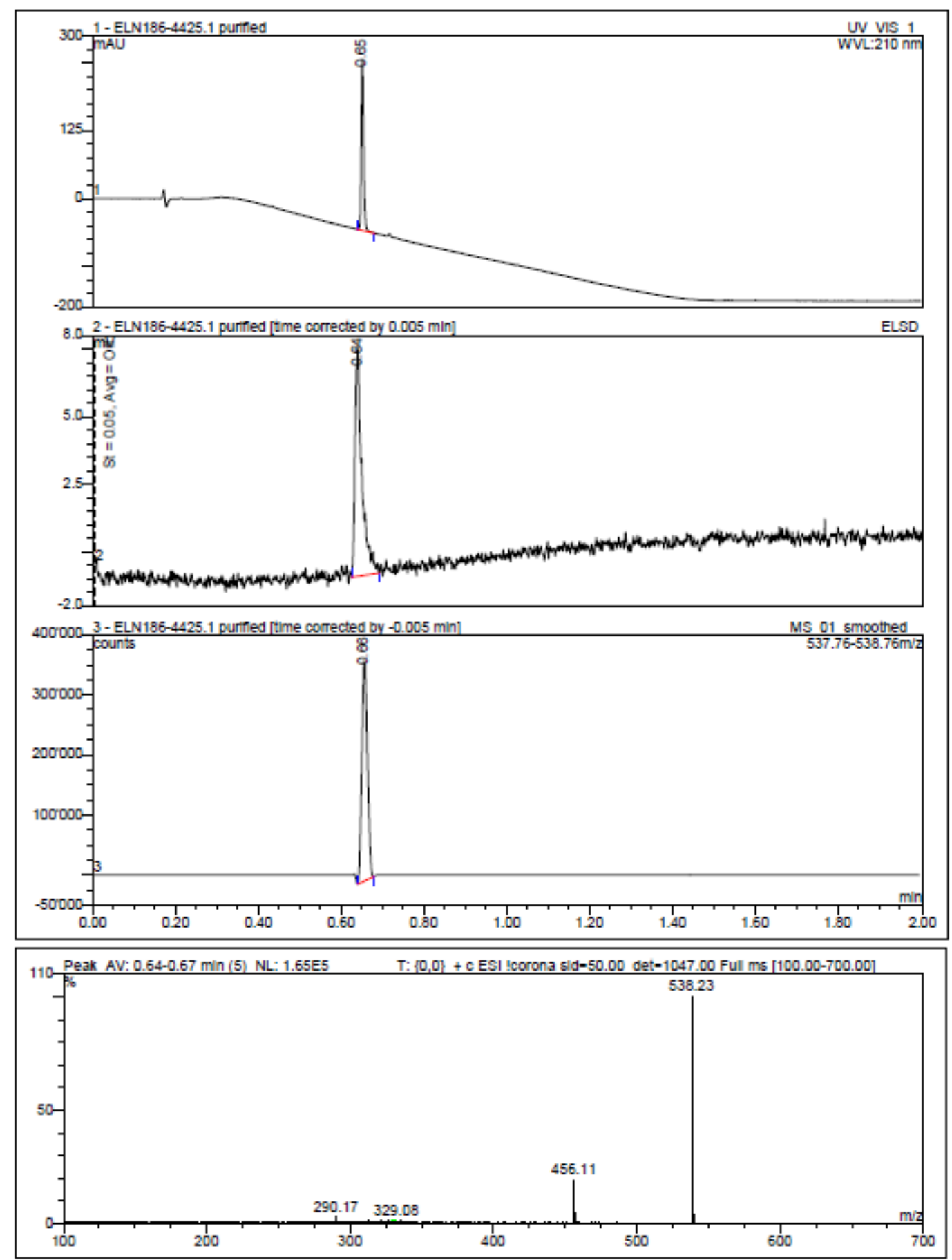


\begin{tabular}{|c|c|c|}
\hline Sample ID: & 048242ea-679b-11ea-a9dc-4ccc6a34d1c7 & \\
\hline Sample Name: & ELN186-4425.1 & Date: 16.03 .20 \\
\hline Sample Number: & 5 & \\
\hline Time Base: & U3000 & \\
\hline Datasource Name: & LCMSCHEM03_local & \\
\hline Sequence Name: & ELN186-4402.1+4425.1 & \\
\hline Sequence Dir. & Datalerharmi1/Samples|" & \\
\hline Quantif. Method: & default & \\
\hline Injection Volume: & 2.50 ul Comment: $1 \mathrm{mg} / \mathrm{mL}$ Heptan/Ethanol 1:1 & \\
\hline Eluent $A$ : & $90.0 \%$ Heptane $0.05 \%$ DEA & \\
\hline Eluent $B$ : & $10.0 \%$ Ethanol $0.05 \%$ DEA & \\
\hline Flow: & $0.800 \mathrm{ml} / \mathrm{min}$ & \\
\hline $\begin{array}{l}\text { Column: } \\
\text { Serial number }\end{array}$ & $\begin{array}{l}\text { Chiralpak IB } 250 \times 4.6 \mathrm{~mm} \text { ID, } 5 u m \\
\text { IB00CE-LI036 }\end{array}$ & \\
\hline Temperature: & $25.0^{\circ} \mathrm{C}$ & \\
\hline Detection: & $260 \mathrm{~nm}$ & \\
\hline
\end{tabular}

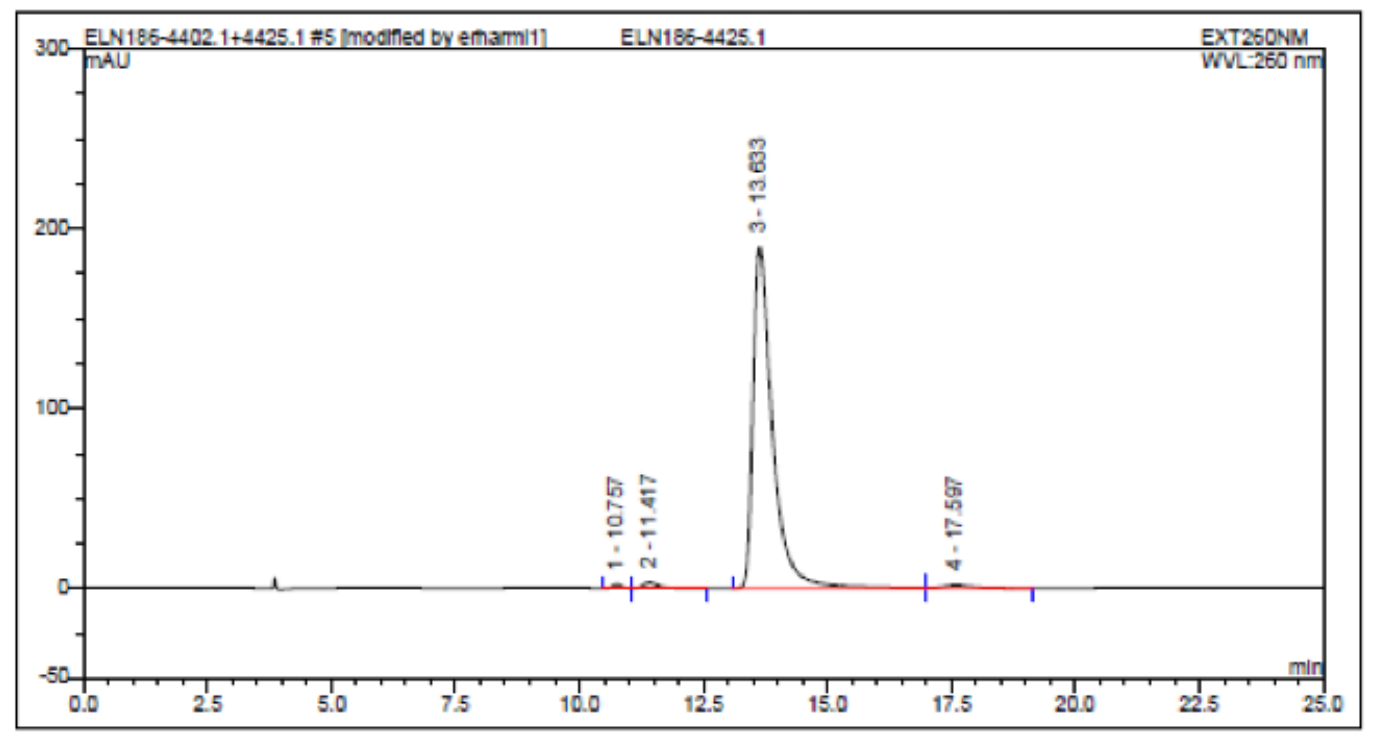

\begin{tabular}{cccccccc}
\hline $\begin{array}{c}\text { Peak No. } \\
\text { EXT260NM }\end{array}$ & $\begin{array}{c}\text { Ret.Time } \\
\text { EXT260NM EXT260NMEXT260NMEXT260NM } \\
\text { min }\end{array}$ & $\begin{array}{c}\text { Height } \\
\text { mAU }\end{array}$ & $\begin{array}{c}\text { Rel.Area } \\
\text { m }\end{array}$ & $\begin{array}{c}\text { Area } \\
\text { mAU*min }\end{array}$ & $\begin{array}{c}\text { Resolution } \\
\text { EXT260NM }\end{array}$ & $\begin{array}{c}\text { Asymmetry } \\
\text { EXT260NM }\end{array}$ & $\begin{array}{c}\text { Plates } \\
\text { EXT260NM }\end{array}$ \\
\hline 1 & 10.8 & 2 & 0.6 & 0.549 & 1.5 & n.a. & 16378 \\
2 & 11.4 & 3 & 1.5 & 1.310 & 3.6 & 1.4 & 6486 \\
3 & 13.6 & 190 & 96.5 & 87.114 & 4.8 & 1.9 & 6947 \\
4 & 17.6 & 2 & 1.4 & 1.302 & n.a. & n.a. & 4873 \\
\hline Total: & & & 100.0 & 90.3 & & & \\
\hline
\end{tabular}


Compound 20q:
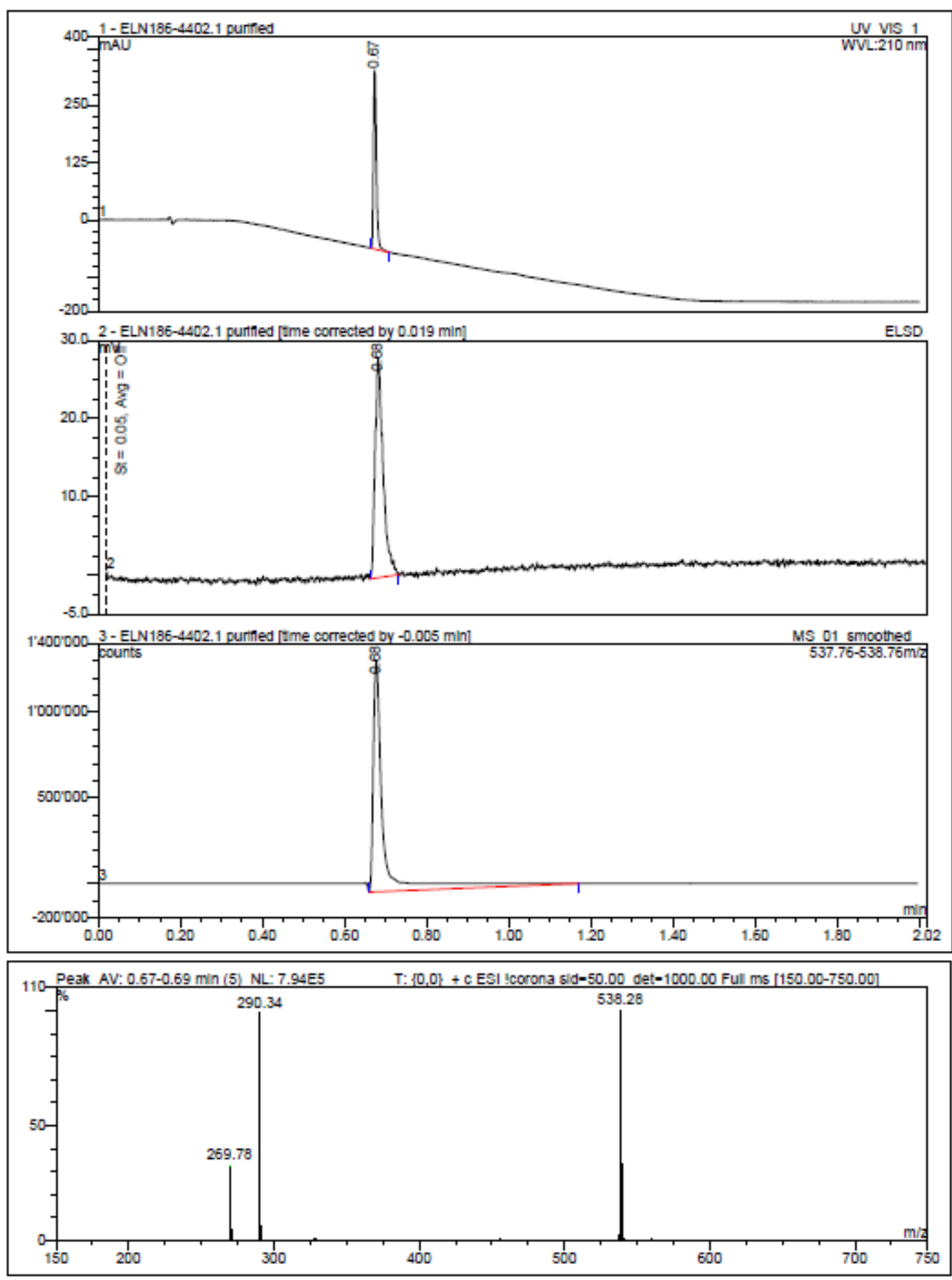


\begin{tabular}{|c|c|c|}
\hline Sample ID: & da238cdf-679e-11ea-a9dc-4ccc6a34d1c7 & \\
\hline Sample Name: & ELN186-4402.1 & Date: 16.03 .20 \\
\hline Sample Number: & 6 & \\
\hline Time Base: & U3000 & \\
\hline Datasource Name: & LCMSCHEM03_local & \\
\hline Sequence Name: & ELN186-4402.1+4425.1 & \\
\hline Sequence Dir: & Datalerharmi1/Samples ' ' & \\
\hline Quantif. Method: & default & \\
\hline Injection Volume: & 3.00 ul Comment: $1 \mathrm{mg} / \mathrm{mL}$ Heptan/Ethanol 1:1 & \\
\hline Eluent $A$ : & $90.0 \%$ Heptane $0.05 \%$ DEA & \\
\hline $\begin{array}{l}\text { Eluent B: } \\
\text { Flow: }\end{array}$ & $10.0 \%$ Ethanol $0.05 \%$ DEA & \\
\hline Flow: & $0.800 \mathrm{ml} / \mathrm{min}$ & \\
\hline Column: & Chiralpak IB 250x4.6mm ID, 5um & \\
\hline Serial number: & IB00CE-LI036 & \\
\hline Temperature: & $25.0{ }^{\circ} \mathrm{C}$ & \\
\hline Detection: & $260 \mathrm{~nm}$ & \\
\hline
\end{tabular}

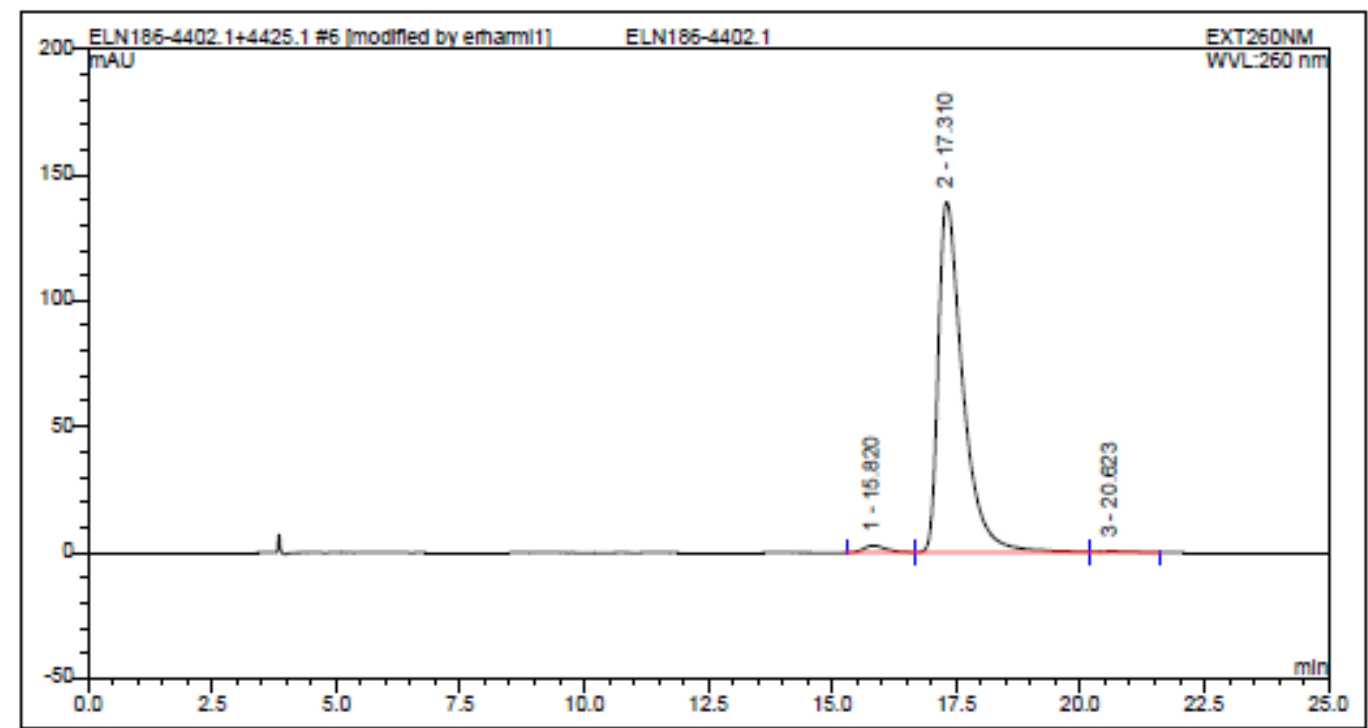

\begin{tabular}{|c|c|c|c|c|c|c|c|}
\hline $\begin{array}{l}\text { Peak No. } \\
\text { EXT260NM }\end{array}$ & $\begin{array}{l}\text { Ret.Time } \\
\text { EXT260NME }\end{array}$ & $\begin{array}{l}\text { Height } \\
\text { EXT260NM }\end{array}$ & $\begin{array}{c}\text { Rel.Area } \\
\text { IEXT260NM }\end{array}$ & $\begin{array}{c}\text { Area } \\
\text { MEXT260NM }\end{array}$ & $\begin{array}{l}\text { Resolution } \\
\text { EXT260NM }\end{array}$ & $\begin{array}{l}\text { Asymmetry } \\
\text { EXT260NM }\end{array}$ & $\begin{array}{c}\text { Plates } \\
\text { EXT260NM }\end{array}$ \\
\hline & $\min$ & mAU & $\%$ & $\mathrm{mAU} \mathbf{U}^{*} \mathrm{~min}$ & & & \\
\hline 1 & 15.8 & 3 & 1.8 & 1.500 & 1.8 & 1.5 & 6162 \\
\hline 2 & 17.3 & 139 & 97.8 & 80.085 & n.a. & 1.8 & 6693 \\
\hline 3 & 20.6 & 0 & 0.4 & 0.332 & n.a. & n.a. & n.a. \\
\hline Total: & & & 100.0 & 81.9 & & & \\
\hline
\end{tabular}


Compound 21a:
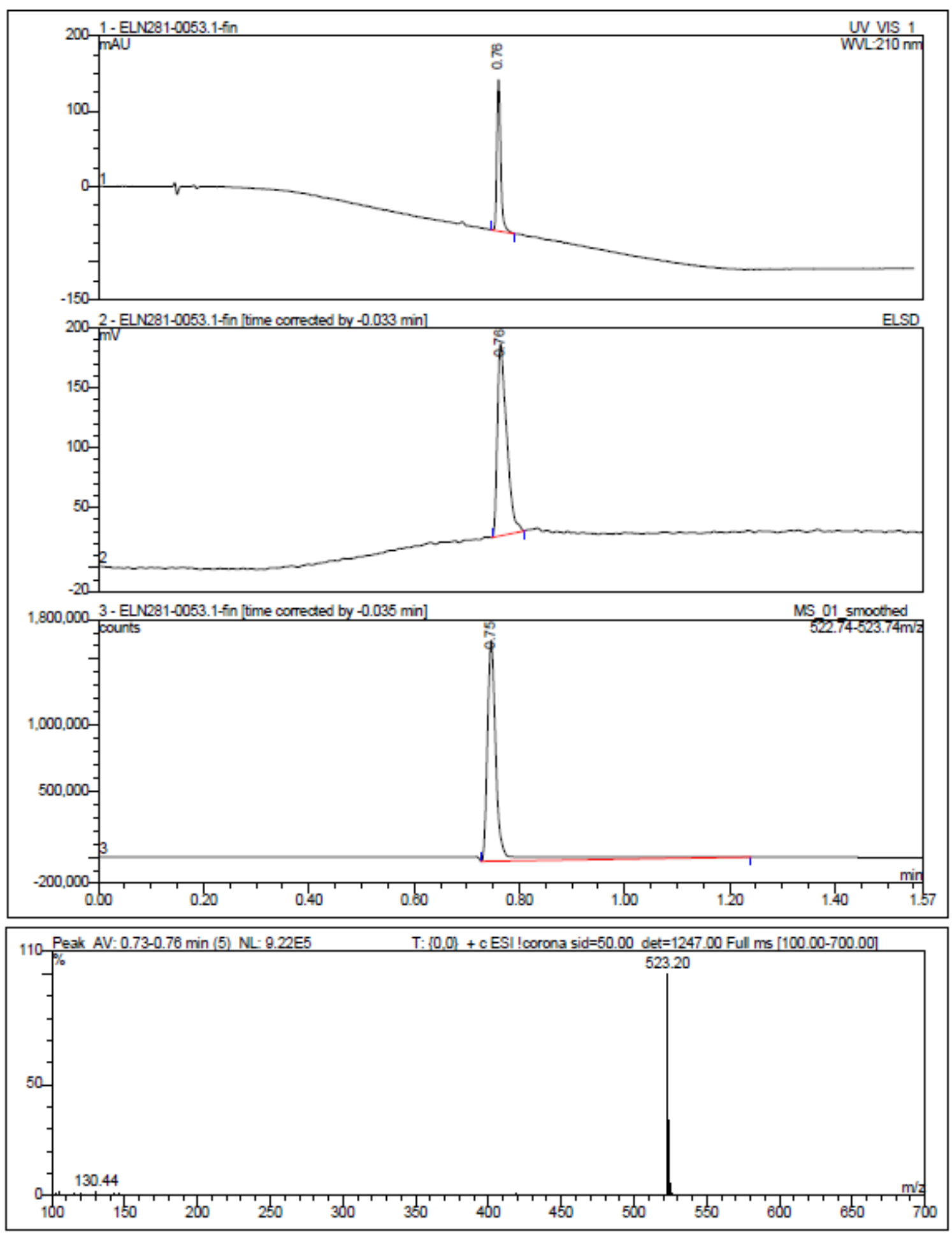
Compound 21b:

Sample Name: LCMSP384-20200430-

1_MO5

\section{Expression}

Auto-Comments

Auto-Sumnary

Purity

Target Mass

Target RT

UV:12216!

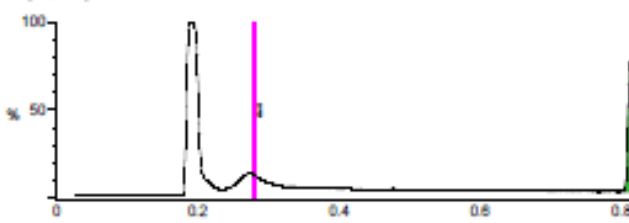

ACT No: ACT-1003-6905

Instrument: ICHALW-DLO0021-SQD
ELN Nr: ELN281-0048.1

Acquisition time: 5/4/2020 3:24 PM

Result

GOOD

96.7000

537.5000

0.8120

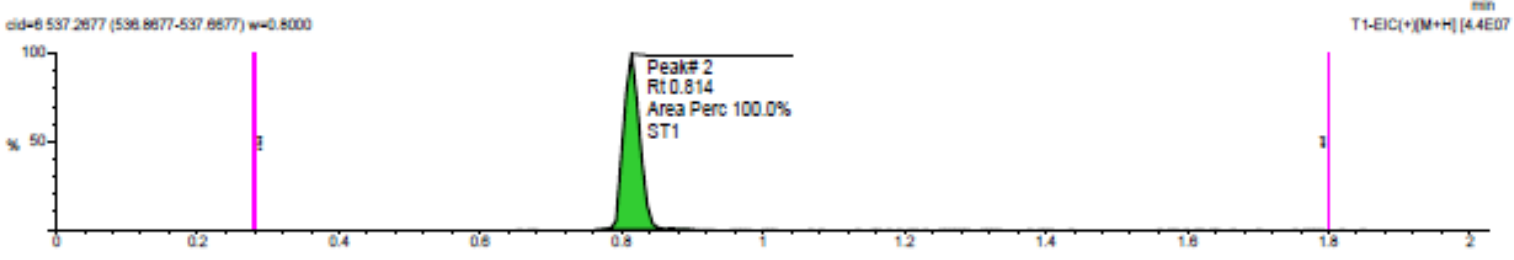

eid-11 5952521 (594 8521.595 es21) w-0.6000

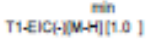

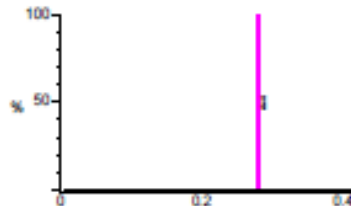

\begin{tabular}{ll} 
Area Perc $96.7 \%$ & Peaki 5 \\
ST1 & Rt 1.229 \\
& Area Perc $2.7 \%$ \\
\hline
\end{tabular}

\begin{tabular}{ll} 
Area Perc $96.7 \%$ & Peak\# 5 \\
ST1 & Rt 1.229 \\
& Area Perc $2.7 \%$ \\
\hline
\end{tabular}

\begin{tabular}{ll} 
Area Perc $96.7 \%$ & Peak\# 5 \\
ST1 & Rt 1.229 \\
& Area Perc $2.7 \%$ \\
\hline
\end{tabular}

UV214 [4 TEOA :

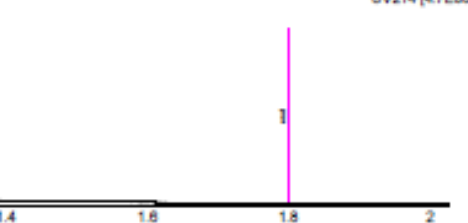

$\min _{\text {in }}$ i

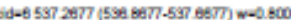

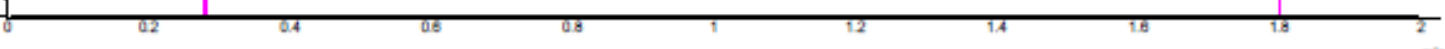

Poak 190.477 min Mo(+) Es

$1.9 E 0$ onte
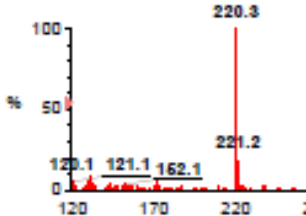

Poak $2 \mathrm{eg} .814 \mathrm{~m} / \mathrm{n}$ wa/*) Es

$3.1 E 07$ onte

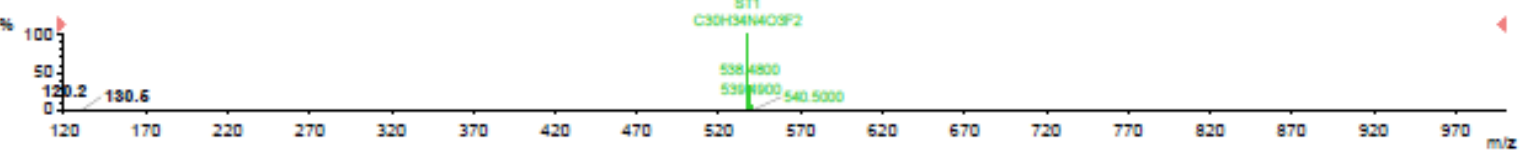


Compound 21c:
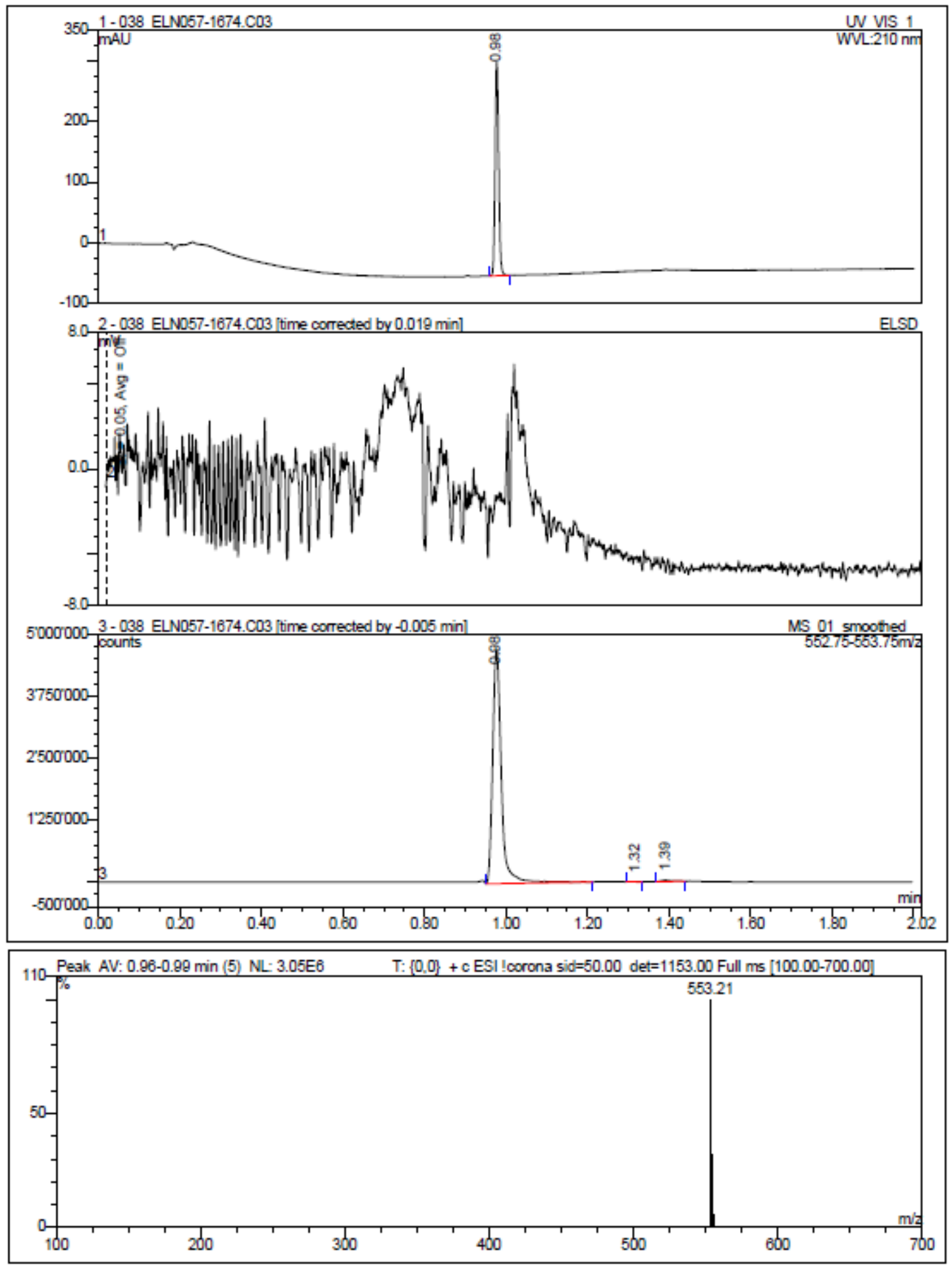
Compound 21d:
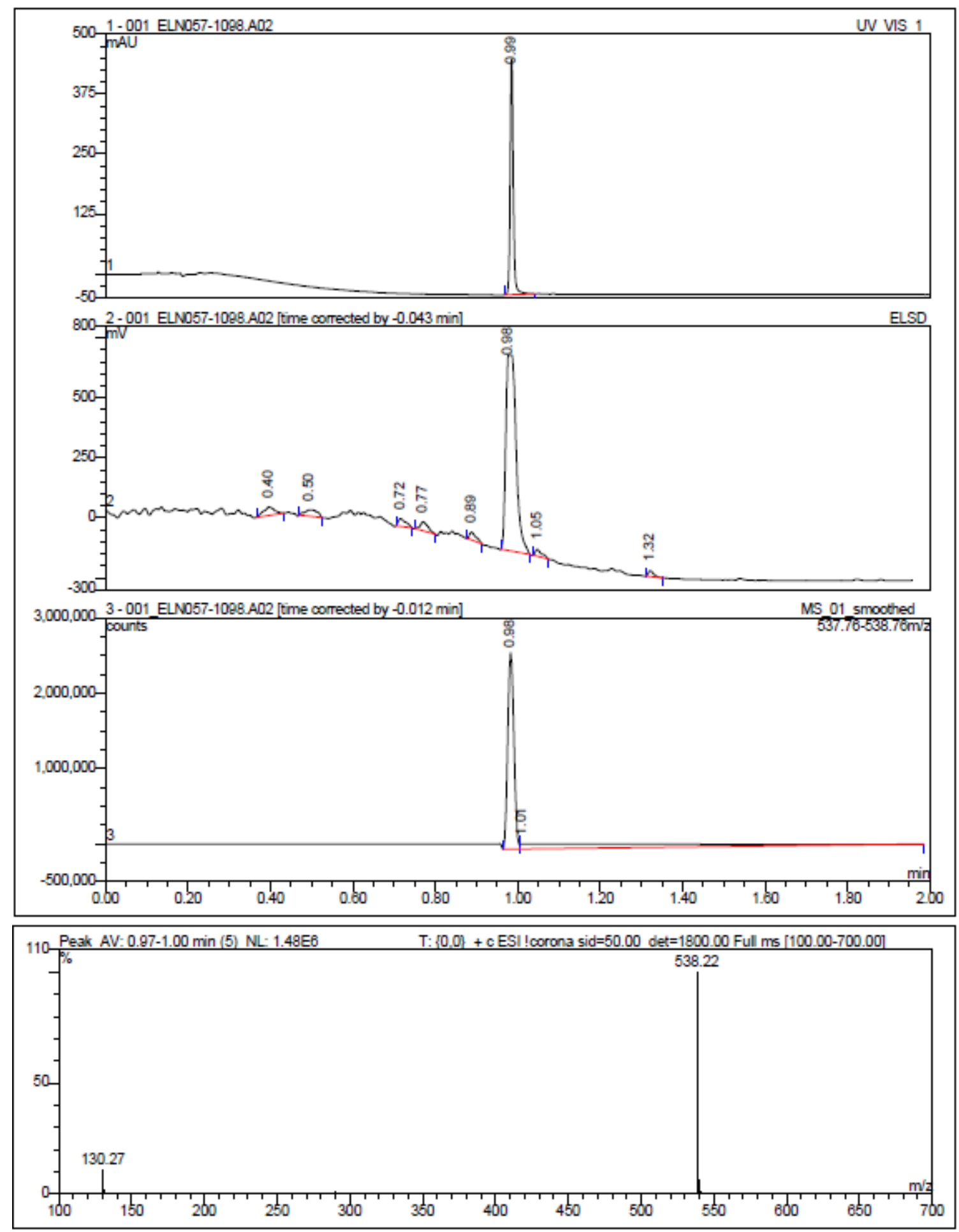
Compound 21d:
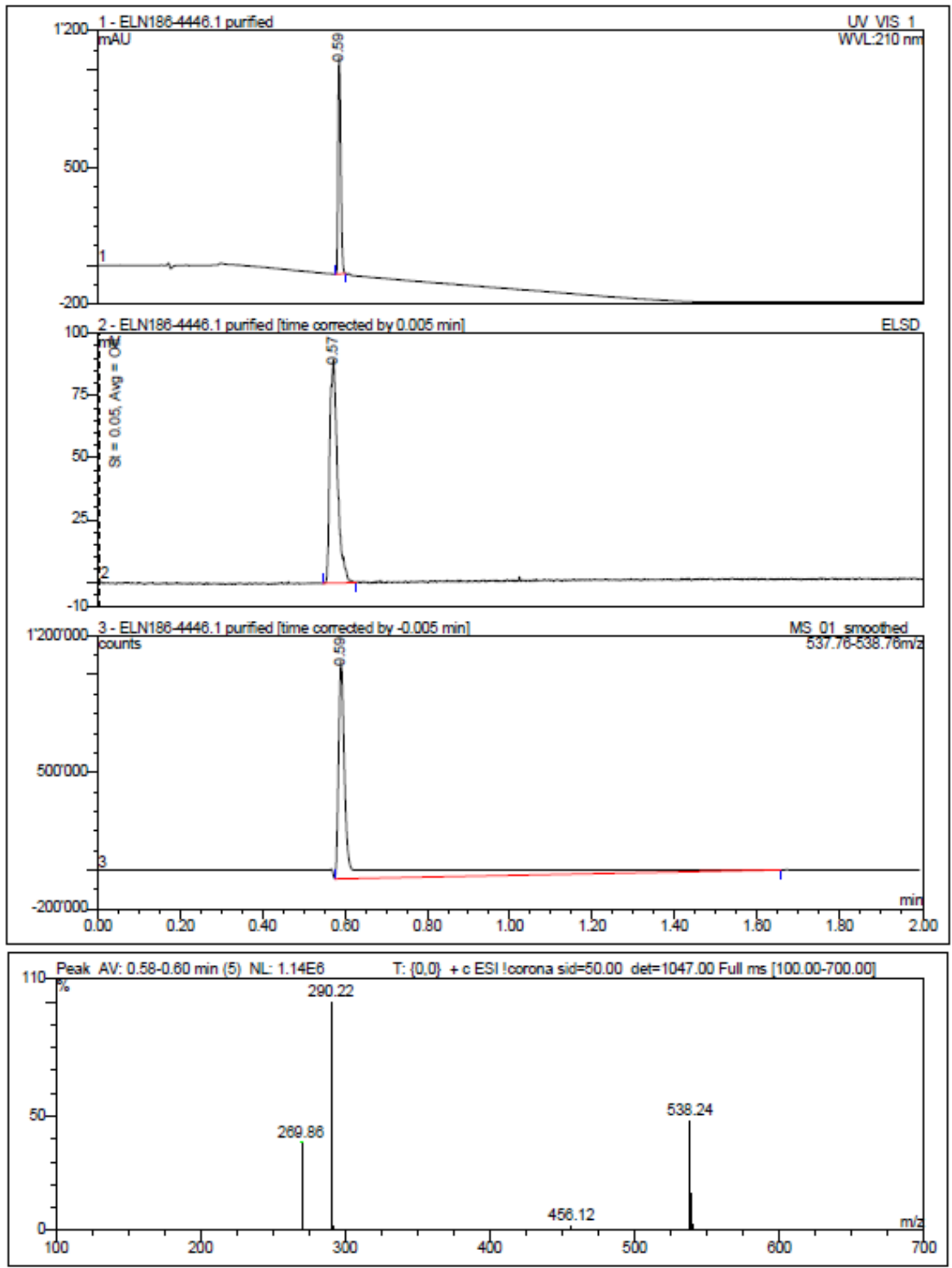
Compound 21e:
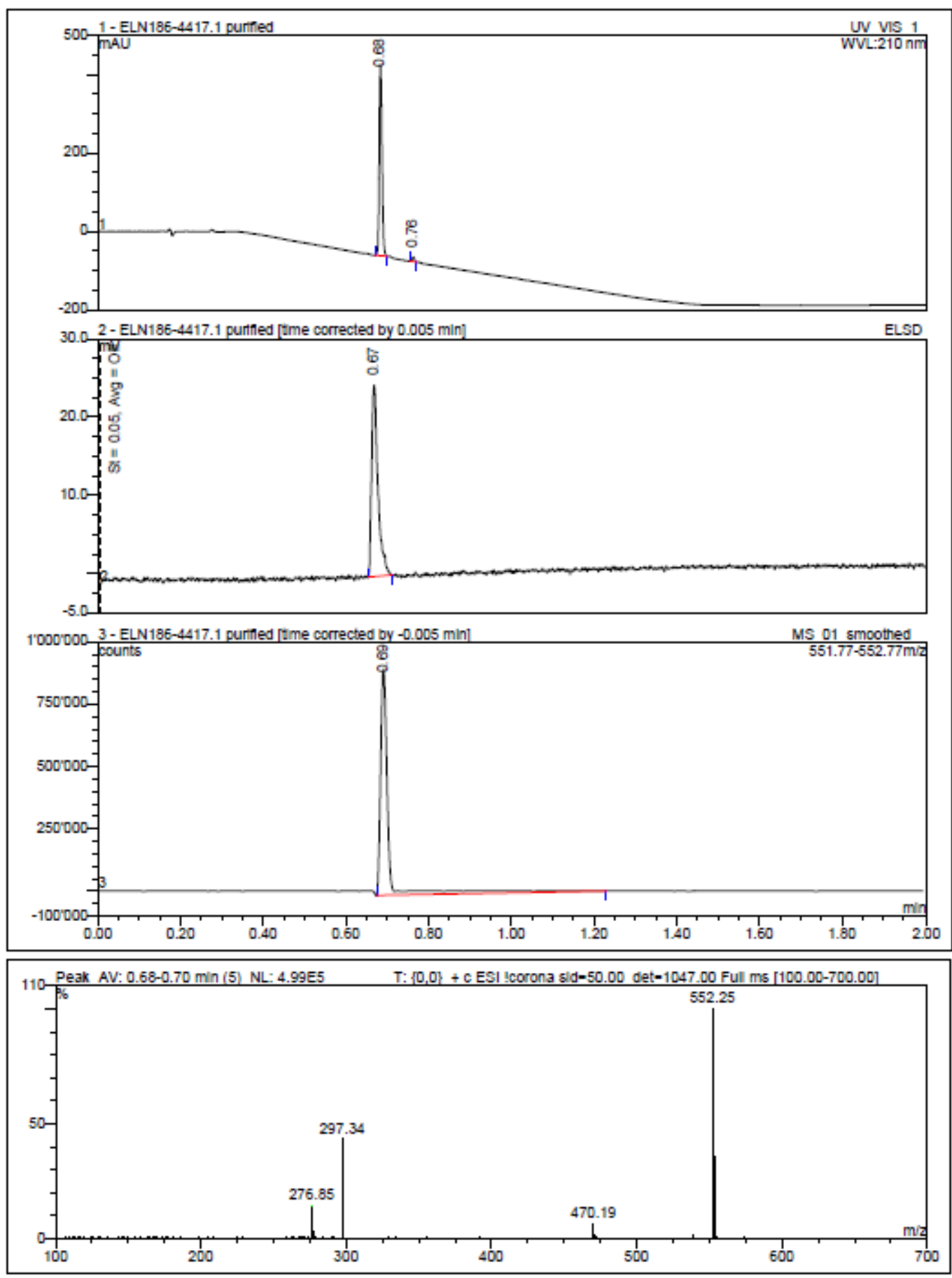

\begin{tabular}{cccccccc}
\hline $\begin{array}{c}\text { Peak No. } \\
\text { UV_VIS_1 }\end{array}$ & $\begin{array}{c}\text { Ret.Time } \\
\text { UV_VIS_1 } \\
\text { min }\end{array}$ & $\begin{array}{c}\text { Area } \\
\text { UV_VIS_1 }\end{array}$ & $\begin{array}{c}\text { Rel.Area } \\
\text { UV_VIS_1 }\end{array}$ & $\begin{array}{c}\text { Mass 01 } \\
\text { UV_VIS_1 } \\
\text { amu }\end{array}$ & $\begin{array}{c}\text { Mass 02 } \\
\text { UV_VIS_1 } \\
\text { amu }\end{array}$ & $\begin{array}{c}\text { Mass 03 } \\
\text { UV_VIS_1 } \\
\text { amu }\end{array}$ & $\begin{array}{c}\text { UV Match } \\
\text { UV_VIS_1 }\end{array}$ \\
\hline 1 & 0.68 & 3821 & 98.1 & 552.25 & 297.34 & 553.24 & 999 \\
2 & 0.76 & 73 & 1.9 & 473.38 & 107.47 & 104.04 & 995 \\
\hline Total: & \multicolumn{7}{c}{100.0} \\
\hline
\end{tabular}


Compound 21f:
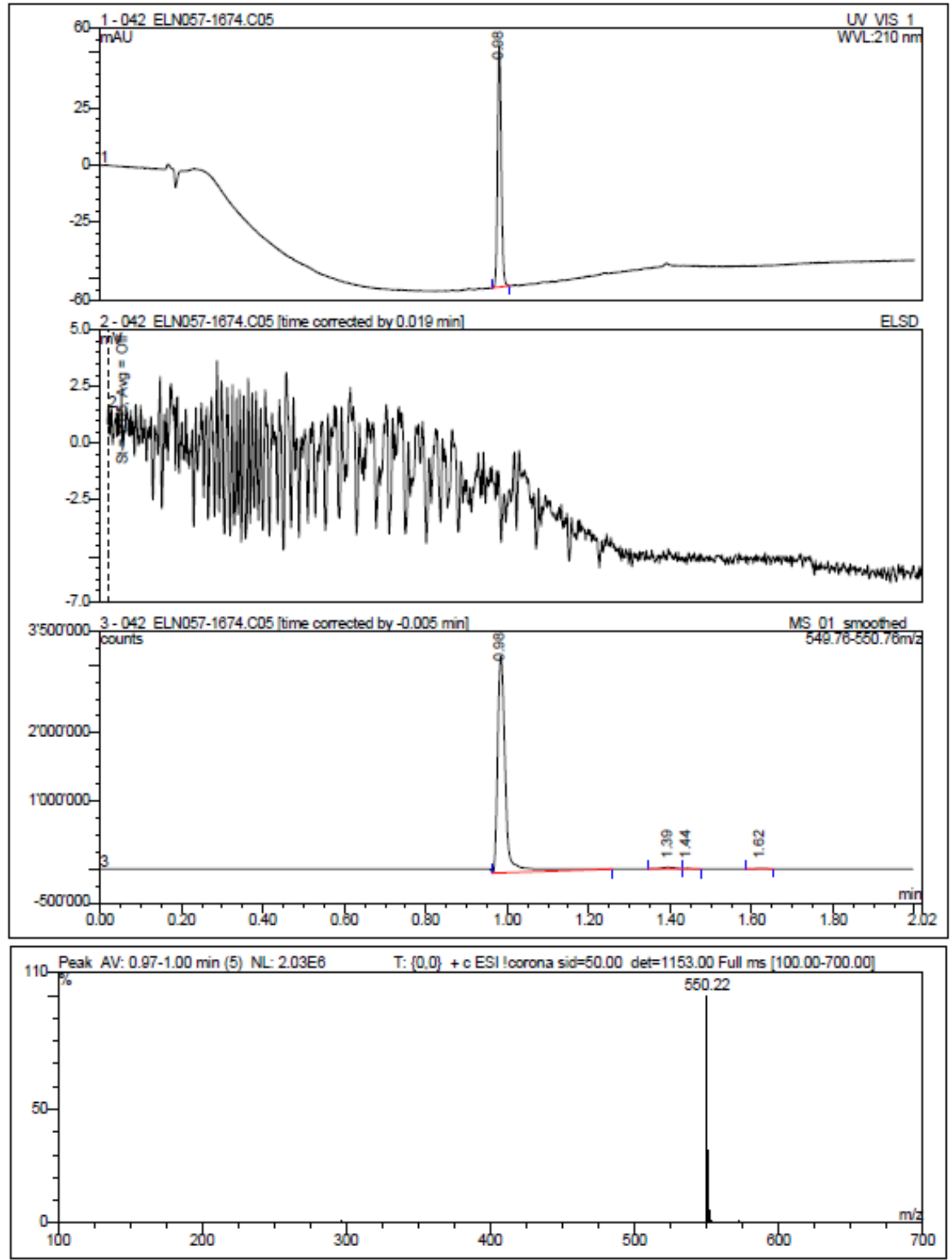
Compound 21g:
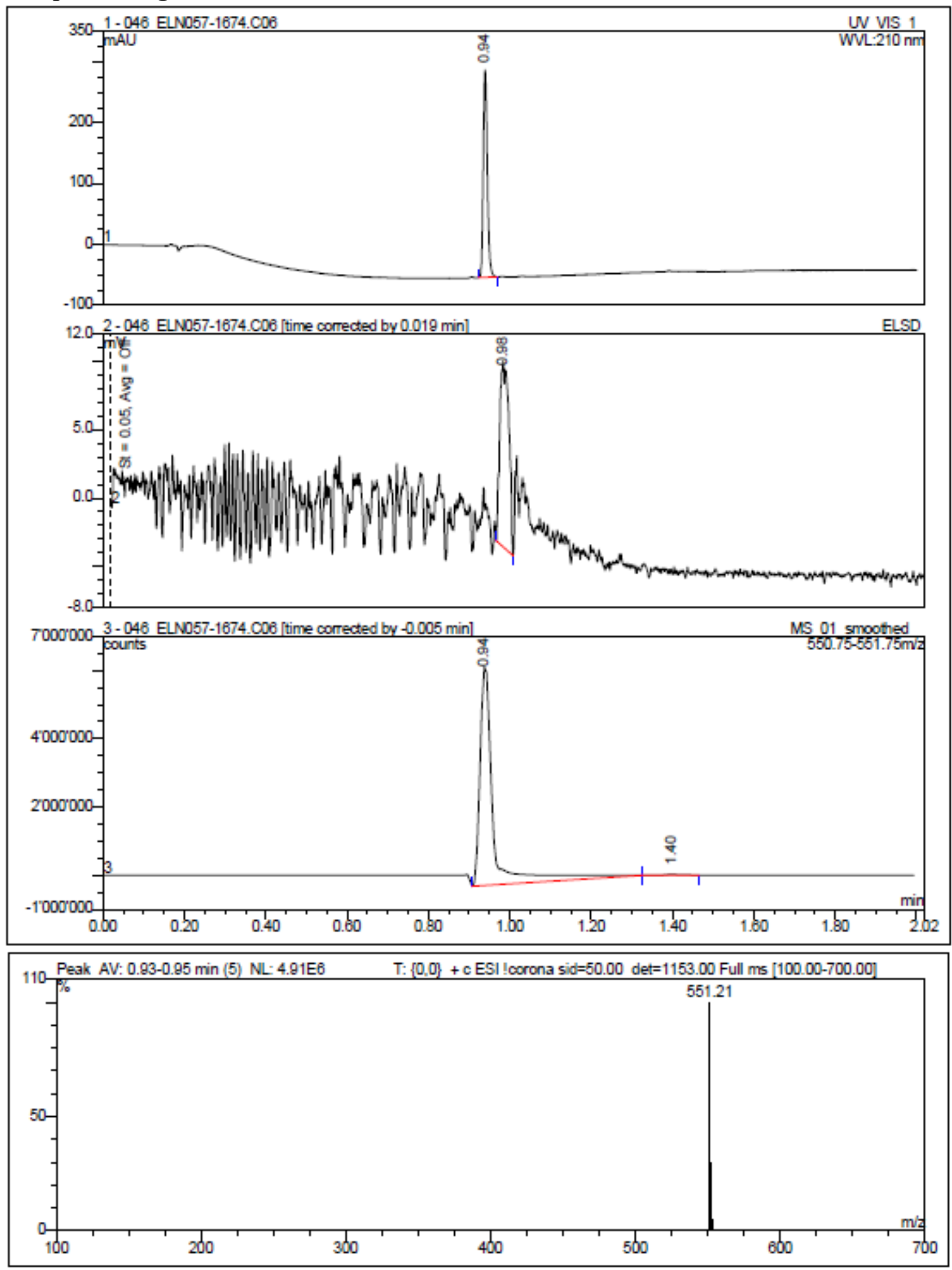
Compound 27:
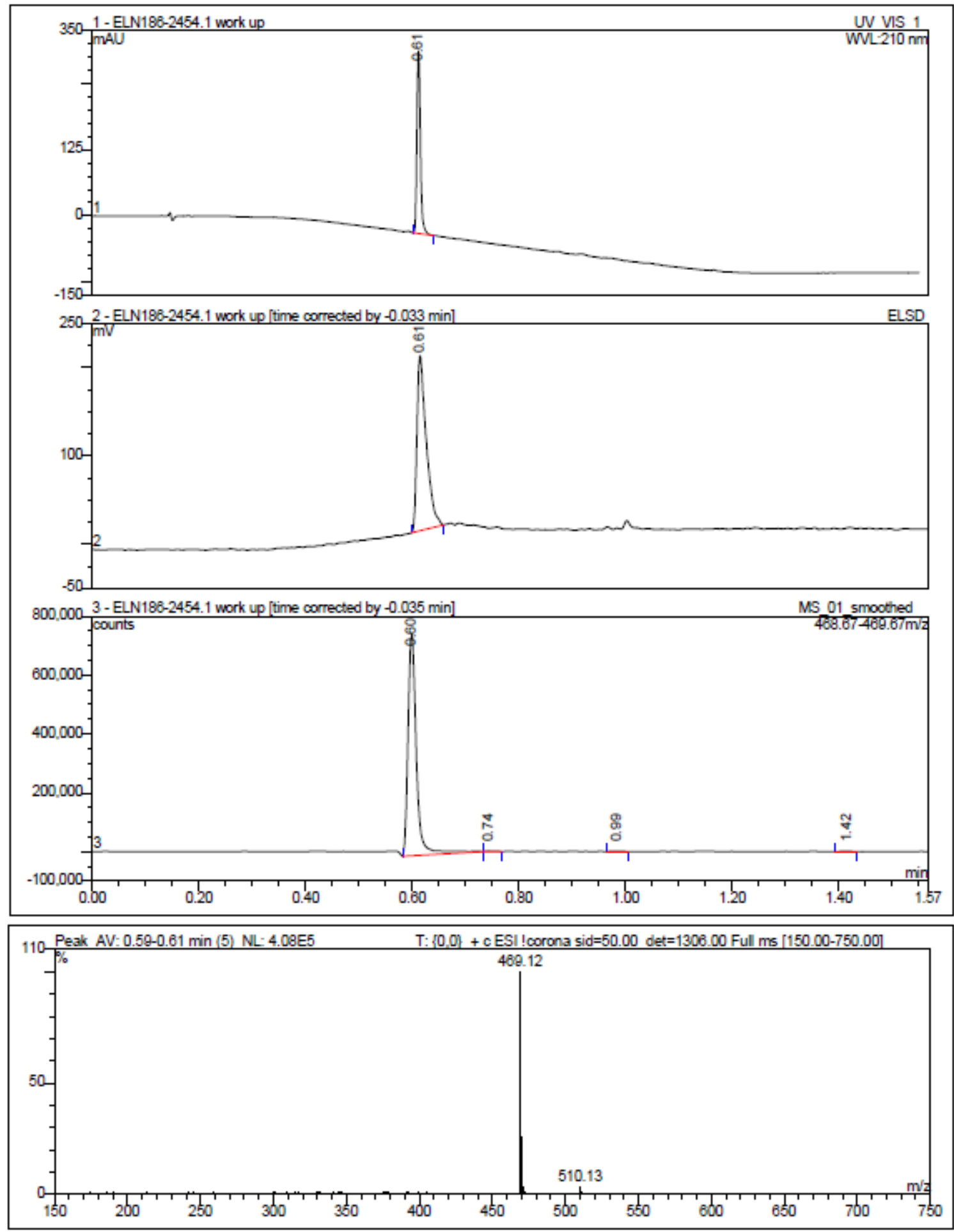
Compound 28a:
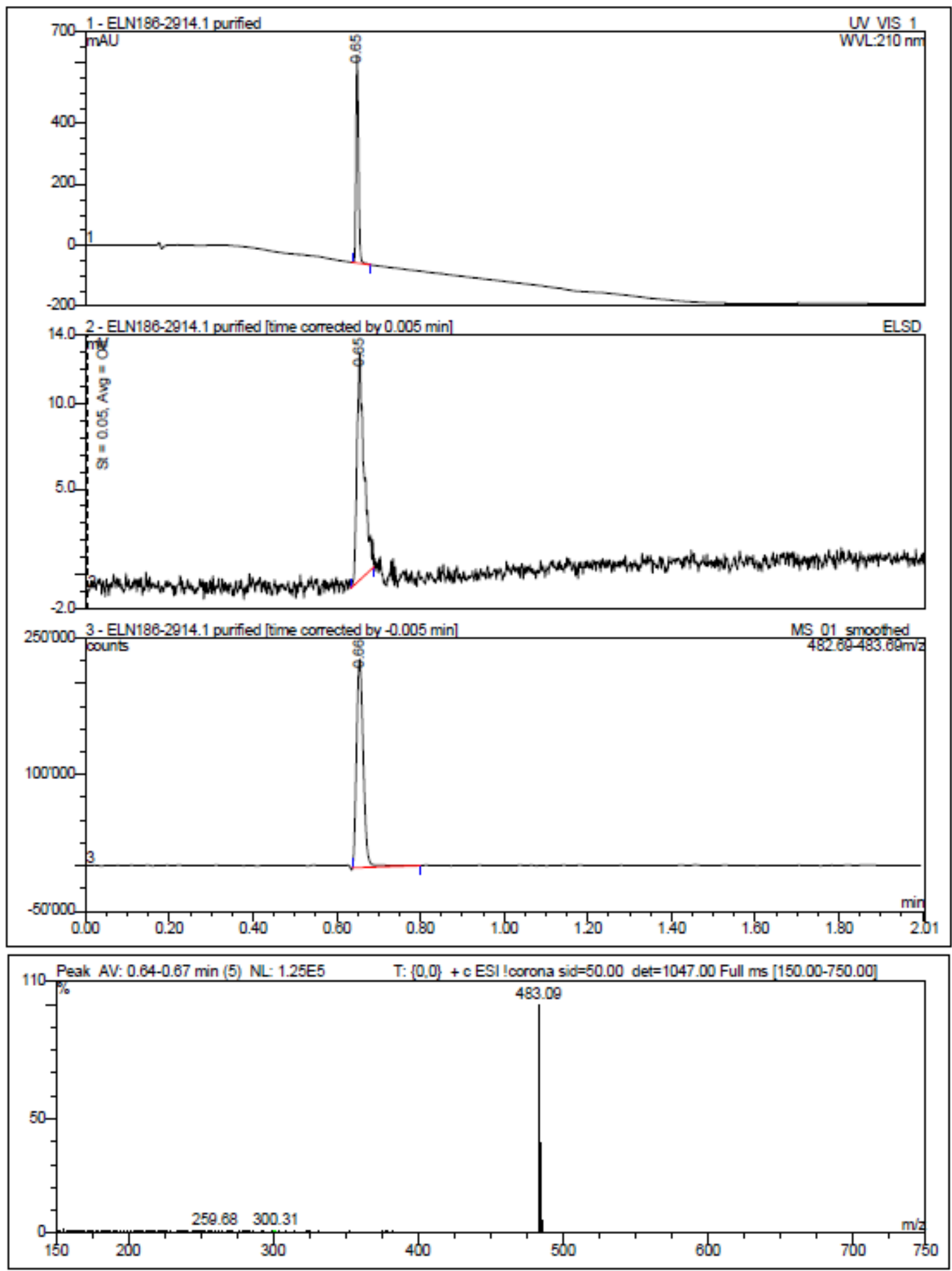
Compound 28b:

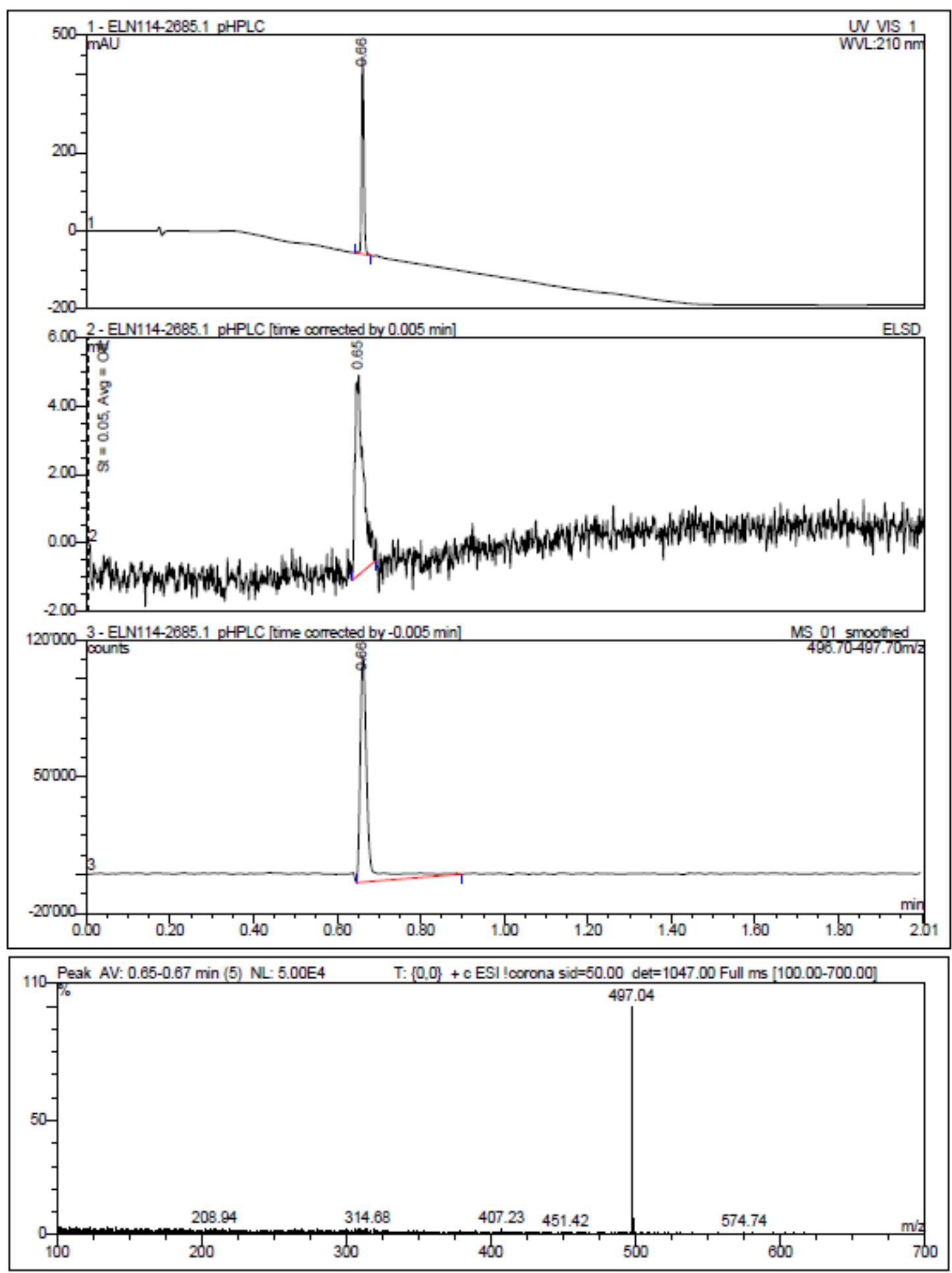


Compound 28c:
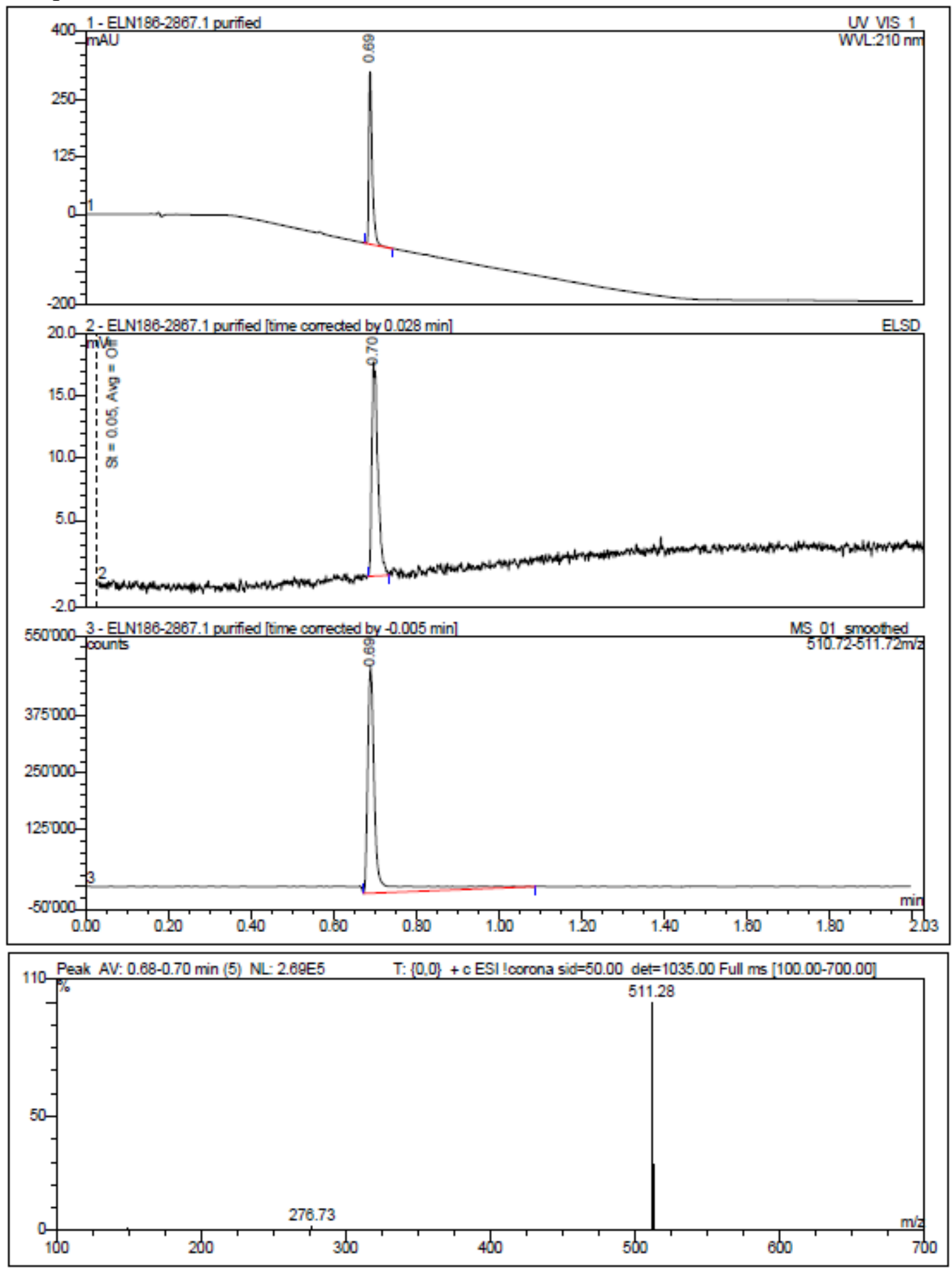
Compound 28d:

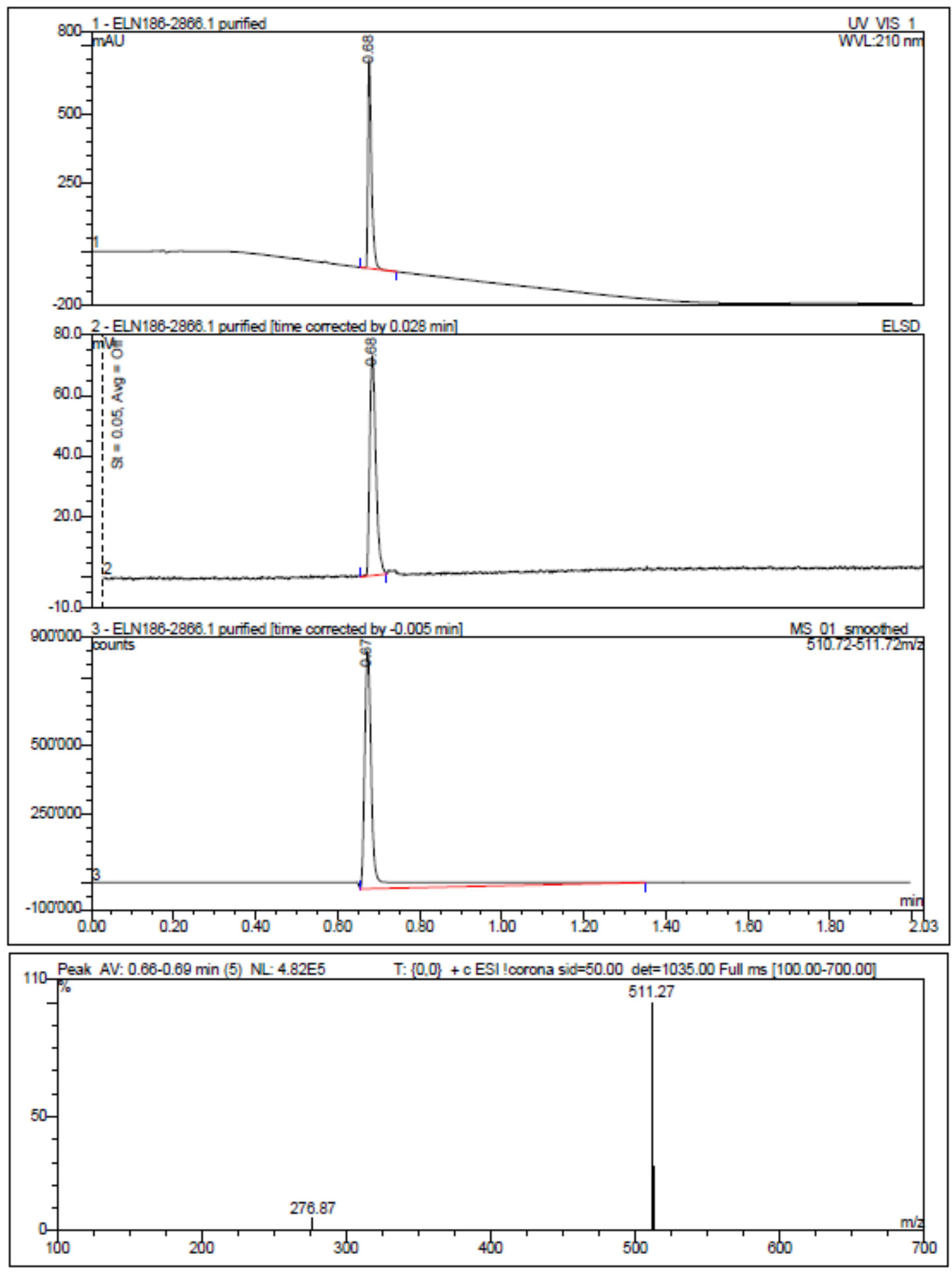


Compound 28e:
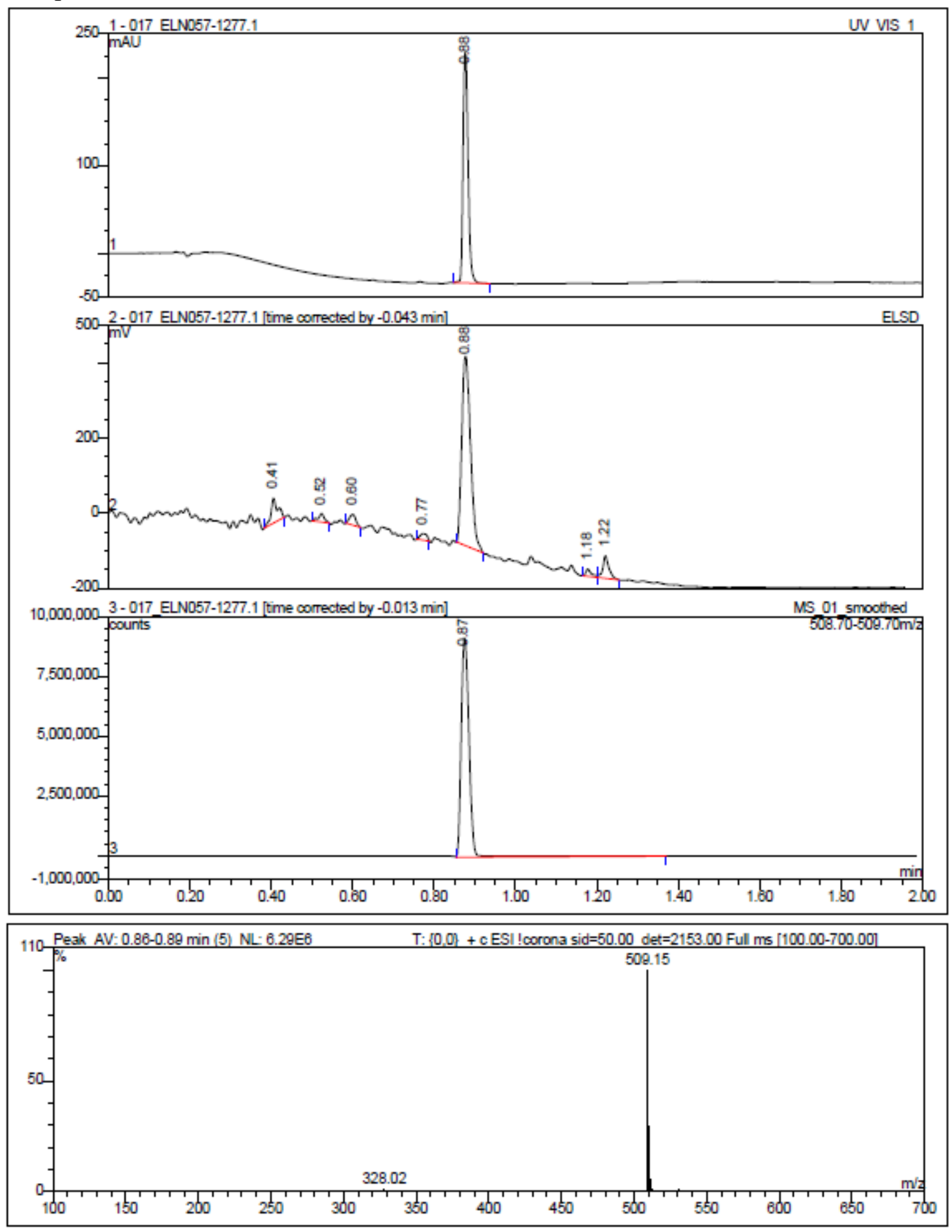


\section{Compound 28f}

Method A
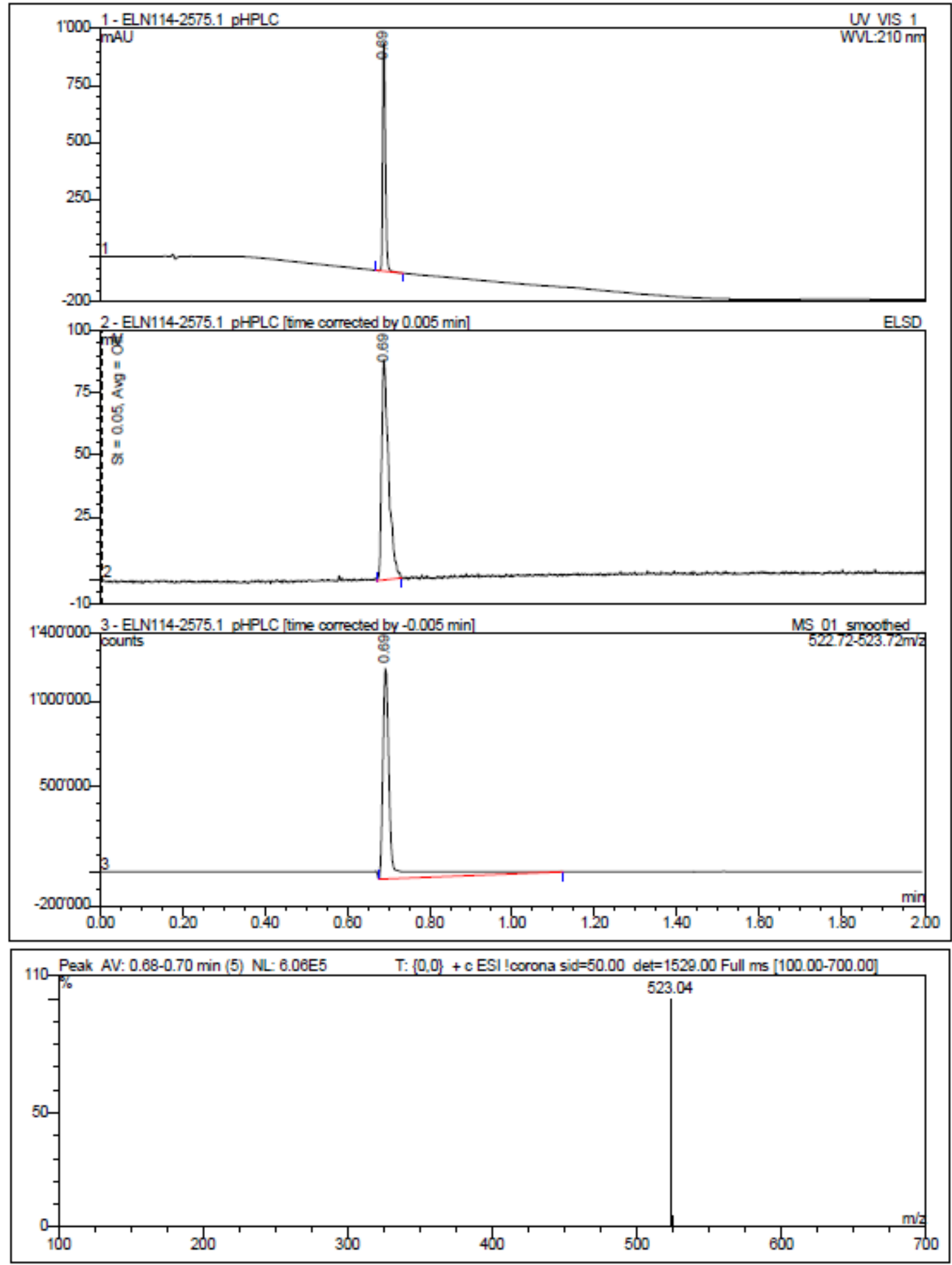


\begin{tabular}{lll|}
\hline Sample ID: & 78d6da9a-9f8c-11e6-ba18-0022191a3ba4 & Date: 31.10 .16 \\
Sample Name: & ELN114-2575.1 & \\
Sample Number: & 3 & \\
Time Base: & U3000 \\
Datasource Name: & LCMSCHEM03_local \\
Sequence Name: & ELN114-2575.1 \\
Sequence Dir: & Datalerharmi1 1 SamplesiCXCR7 \\
Quantif. Method: & default \\
Injection Volume: & $2.00 \mathrm{ul}$ Comment: $1.2 \mathrm{mg} / \mathrm{mL}$ EtOH \\
Eluent A: & $10.0 \%$ Heptane $0.05 \%$ DEA \\
Eluent B: & $90.0 \%$ Ethanol 0.05\% DEA \\
Flow: & $1.200 \mathrm{ml} / \mathrm{min}$ \\
Column: & Chiralpak IC 250x4.6mm ID, 5um \\
Serial number: & IC00CE-OC010 \\
Temperature: & $25.0{ }^{\circ} \mathrm{C}$ \\
Detection: & $210 \mathrm{~nm}$ \\
\hline
\end{tabular}

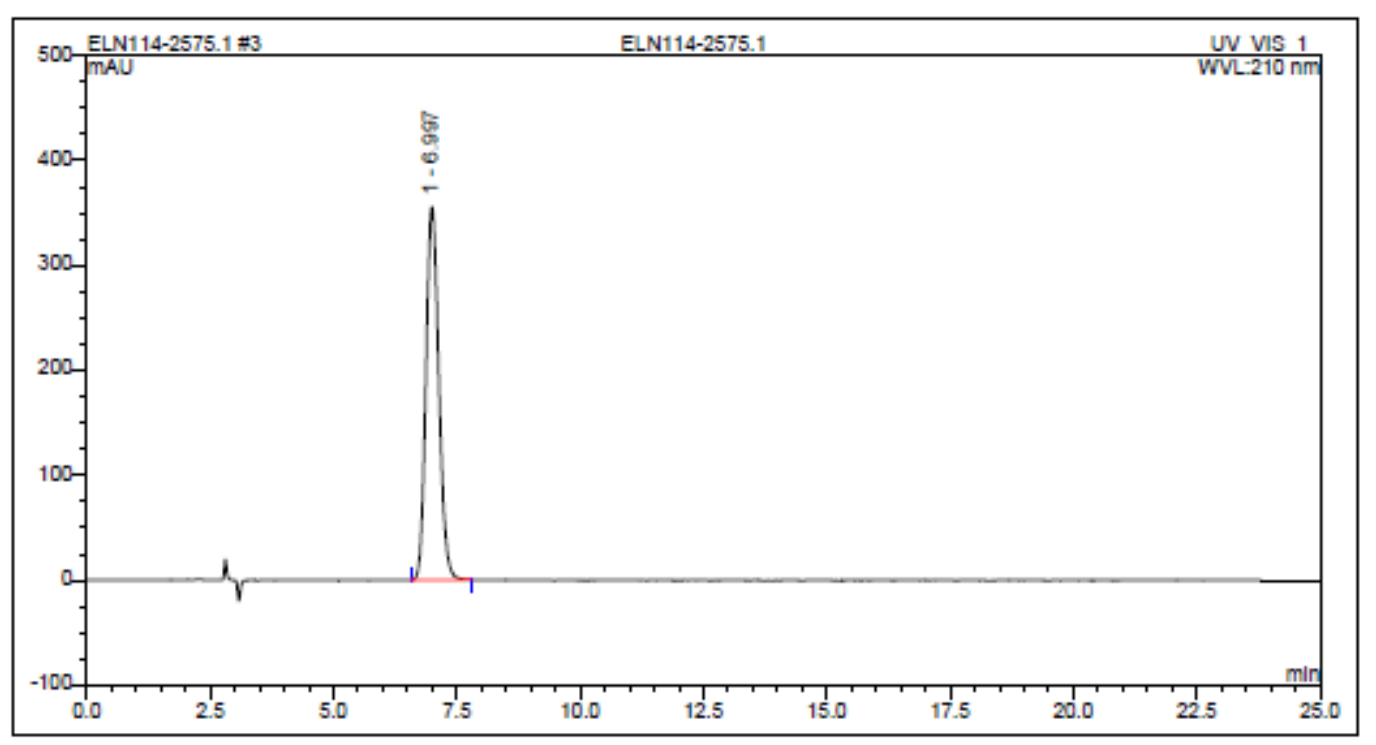

\begin{tabular}{cccccccc}
\hline $\begin{array}{c}\text { Peak No. } \\
\text { UV_VIS_1 }\end{array}$ & $\begin{array}{c}\text { Ret.Time } \\
\text { UV_VIS_1 } \\
\text { min }\end{array}$ & $\begin{array}{c}\text { Height } \\
\text { UV_VIS_1 } \\
\text { mAU }\end{array}$ & $\begin{array}{c}\text { Rel.Area } \\
\text { UV_VIS_1 } \\
\%\end{array}$ & $\begin{array}{c}\text { Area } \\
\text { UV_VIS_1 } \\
\text { mAU*min }\end{array}$ & $\begin{array}{c}\text { Resolution } \\
\text { UV_VIS_1 }\end{array}$ & $\begin{array}{c}\text { Asymmetry } \\
\text { UV_VIS_1 }\end{array}$ & $\begin{array}{c}\text { Plates } \\
\text { UV_VIS_1 }\end{array}$ \\
\hline Total: & 7.0 & 355 & 100.0 & 107.276 & n.a. & 1.1 & 3463 \\
\hline
\end{tabular}


Compound 28g:
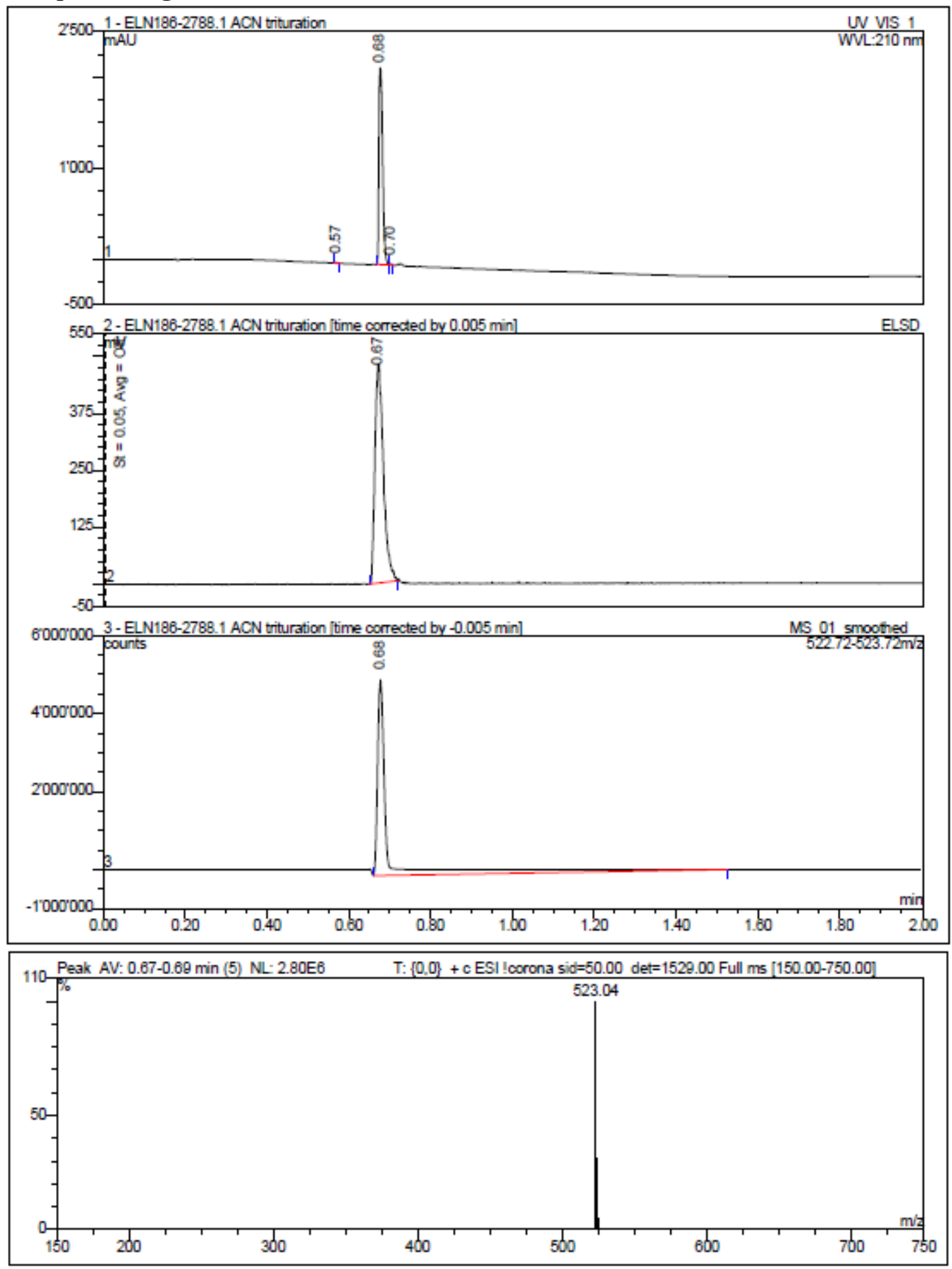
Compound 28h:
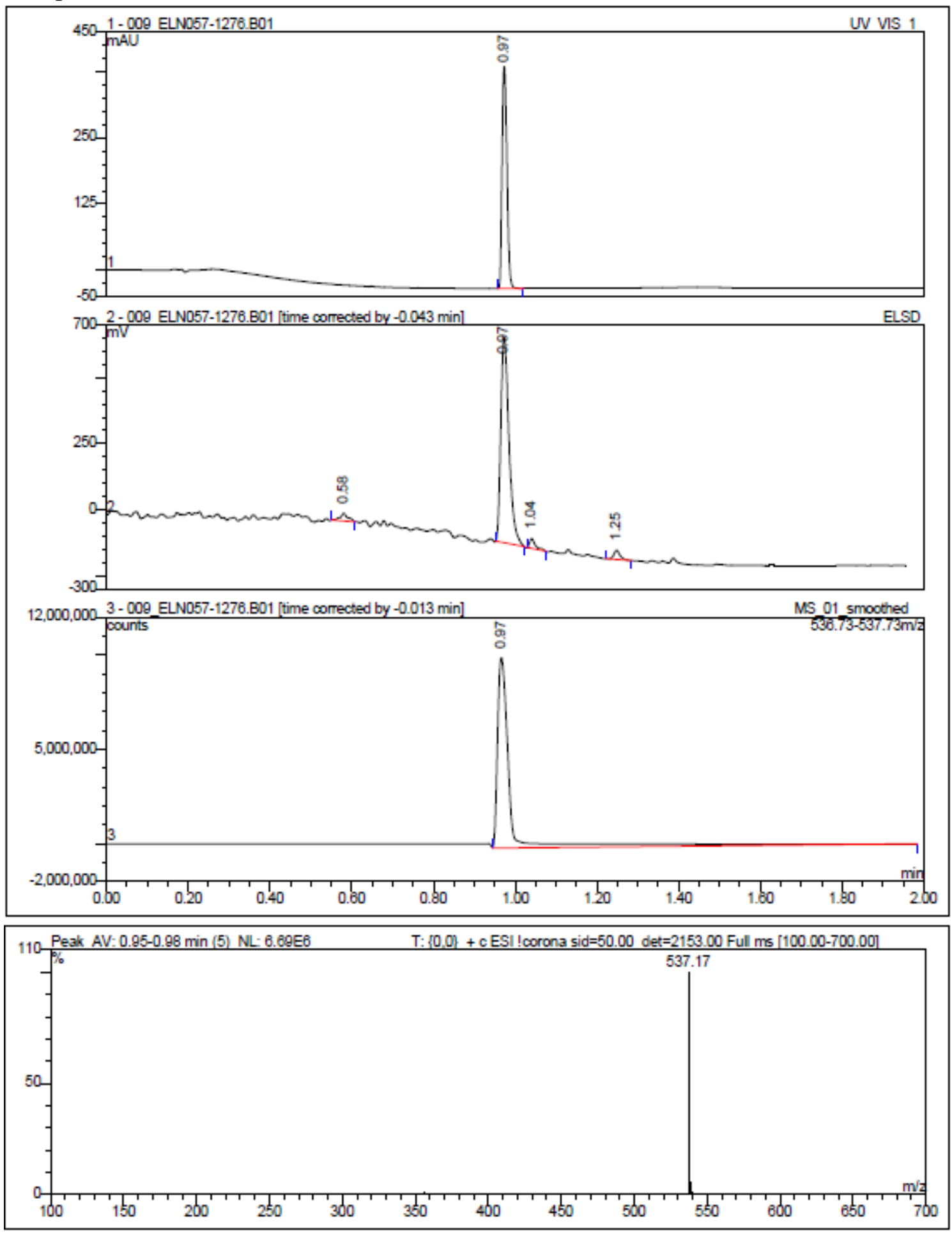
Compound 28i:
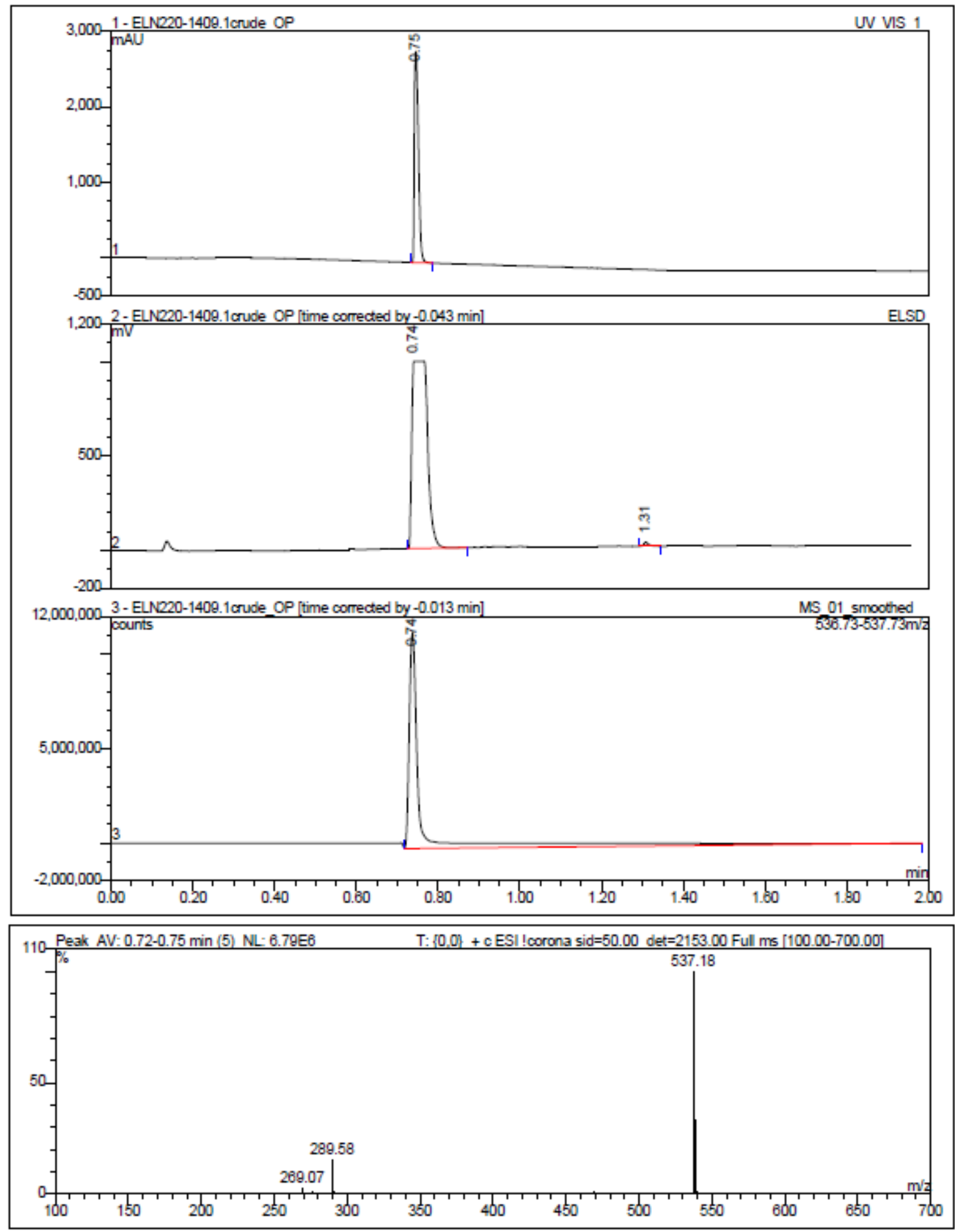
Compound 28j:
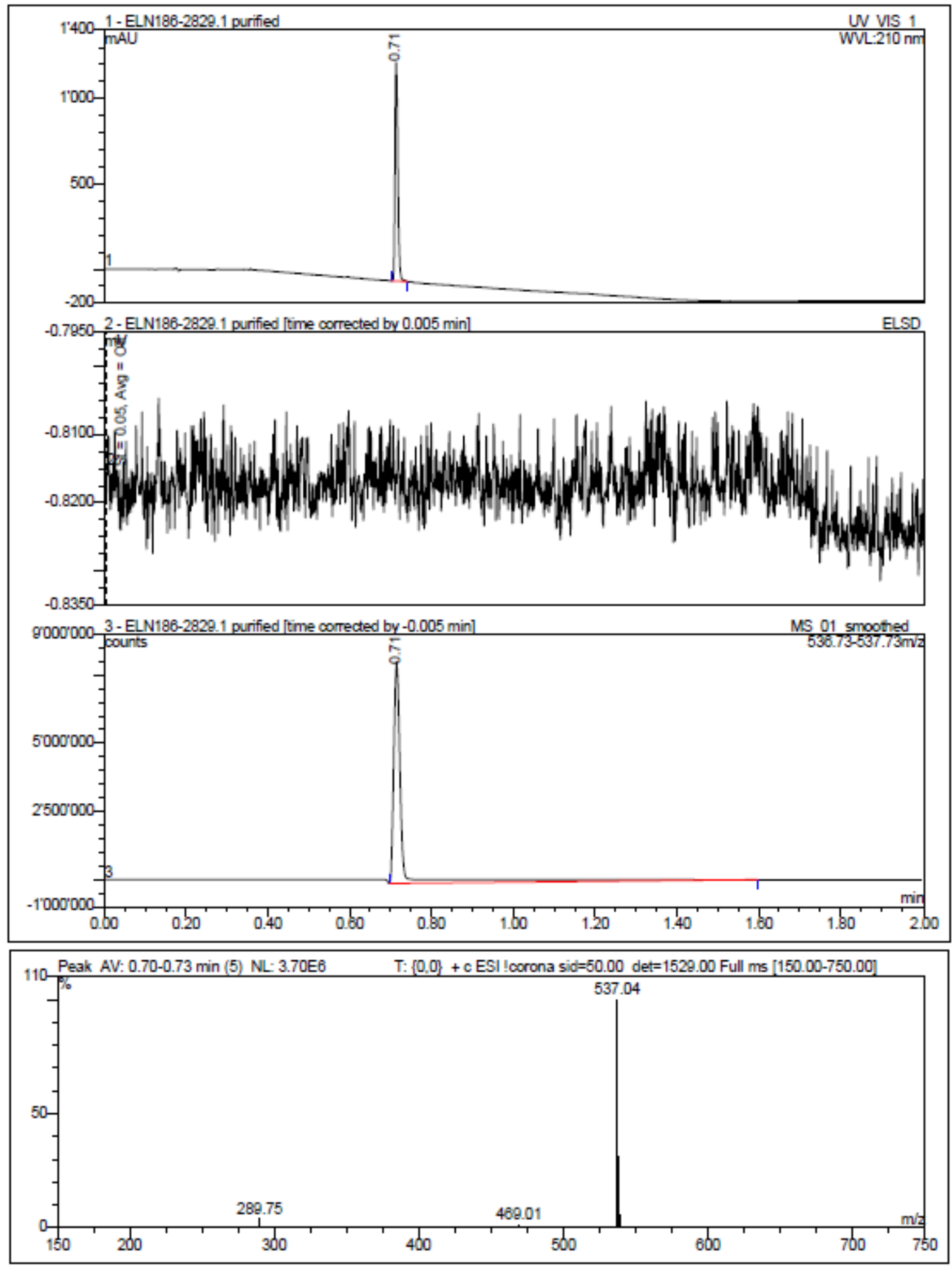
Compound 28k:

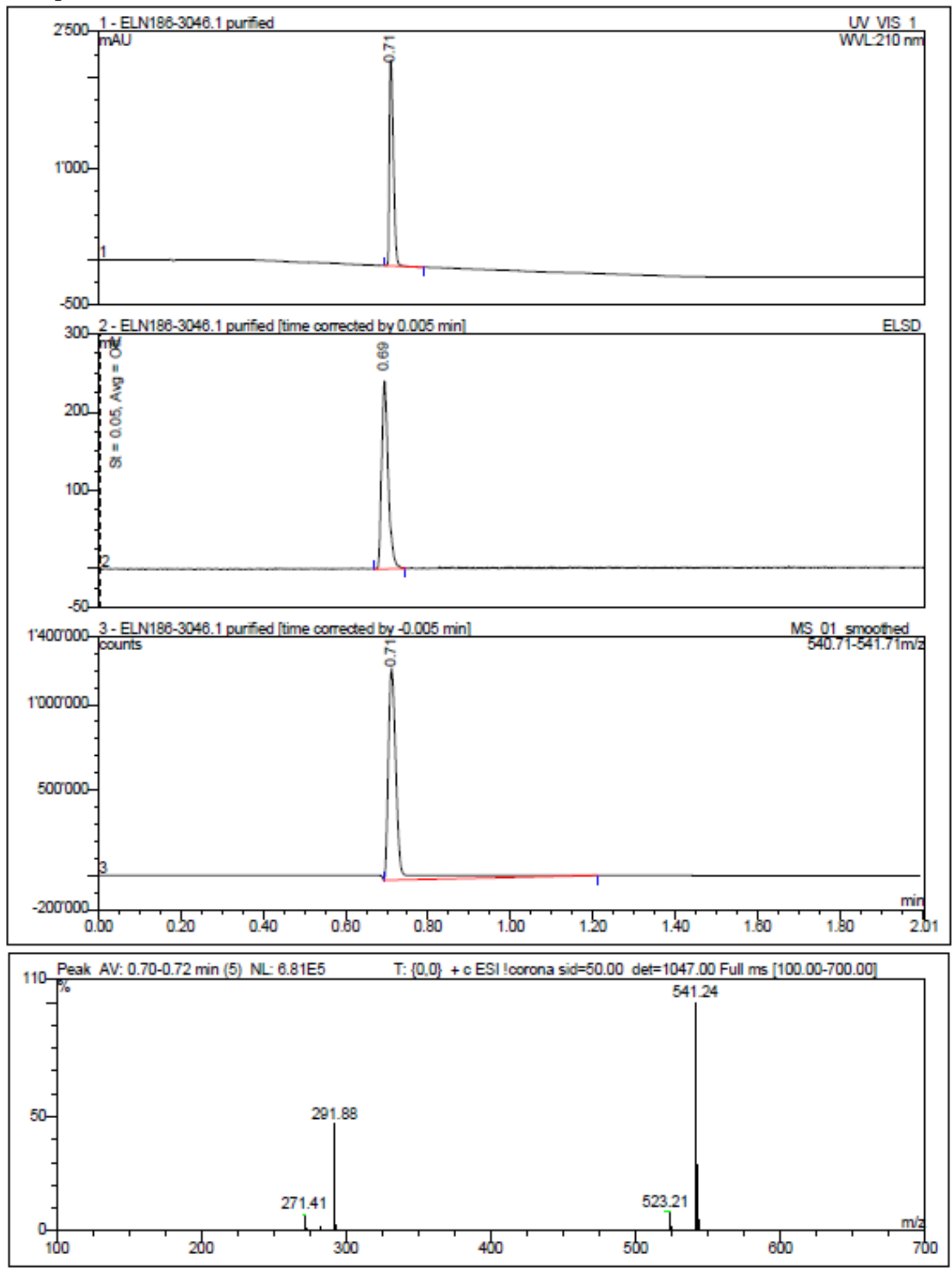


Compound 281:
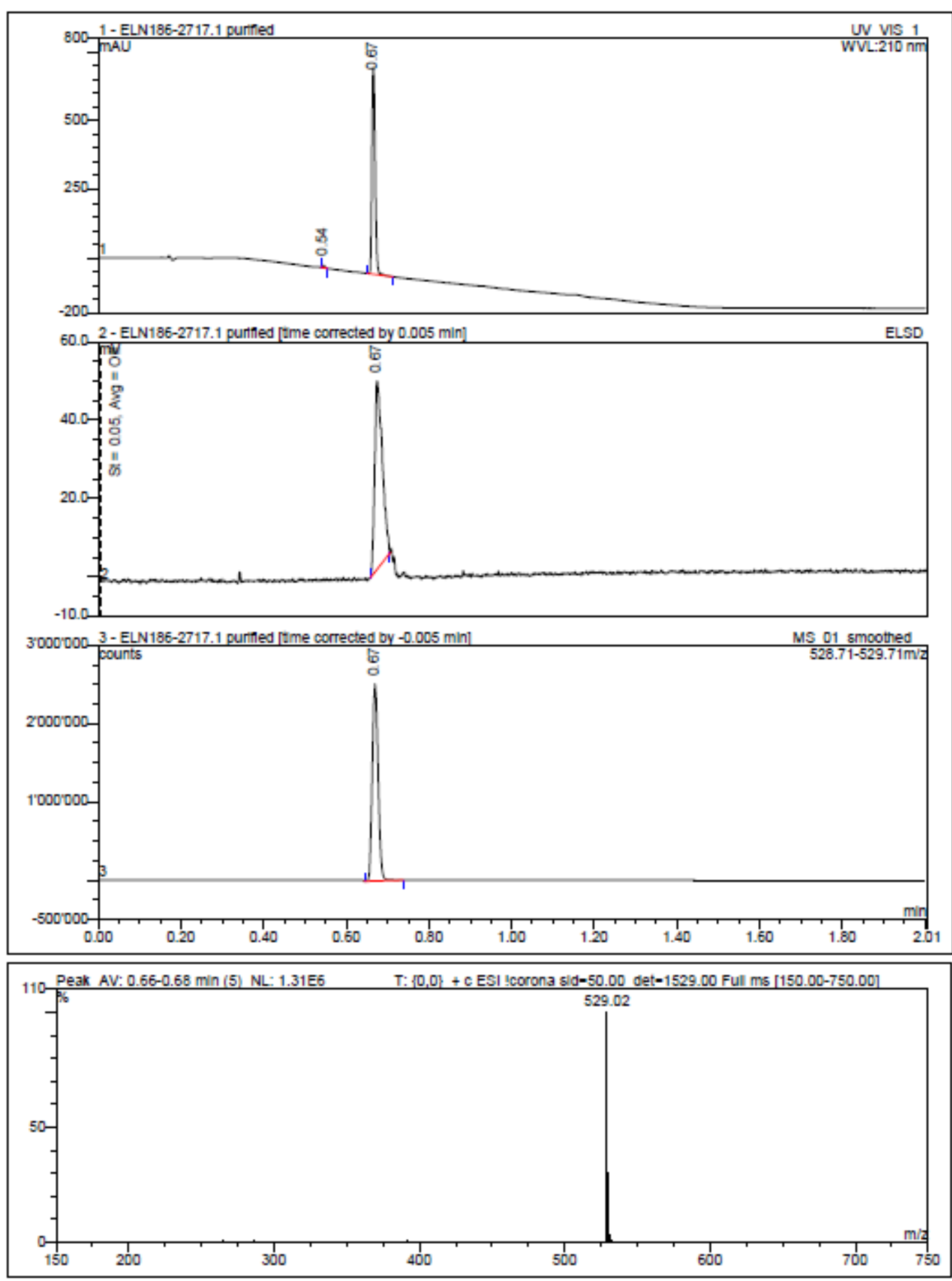
Compound 28m:
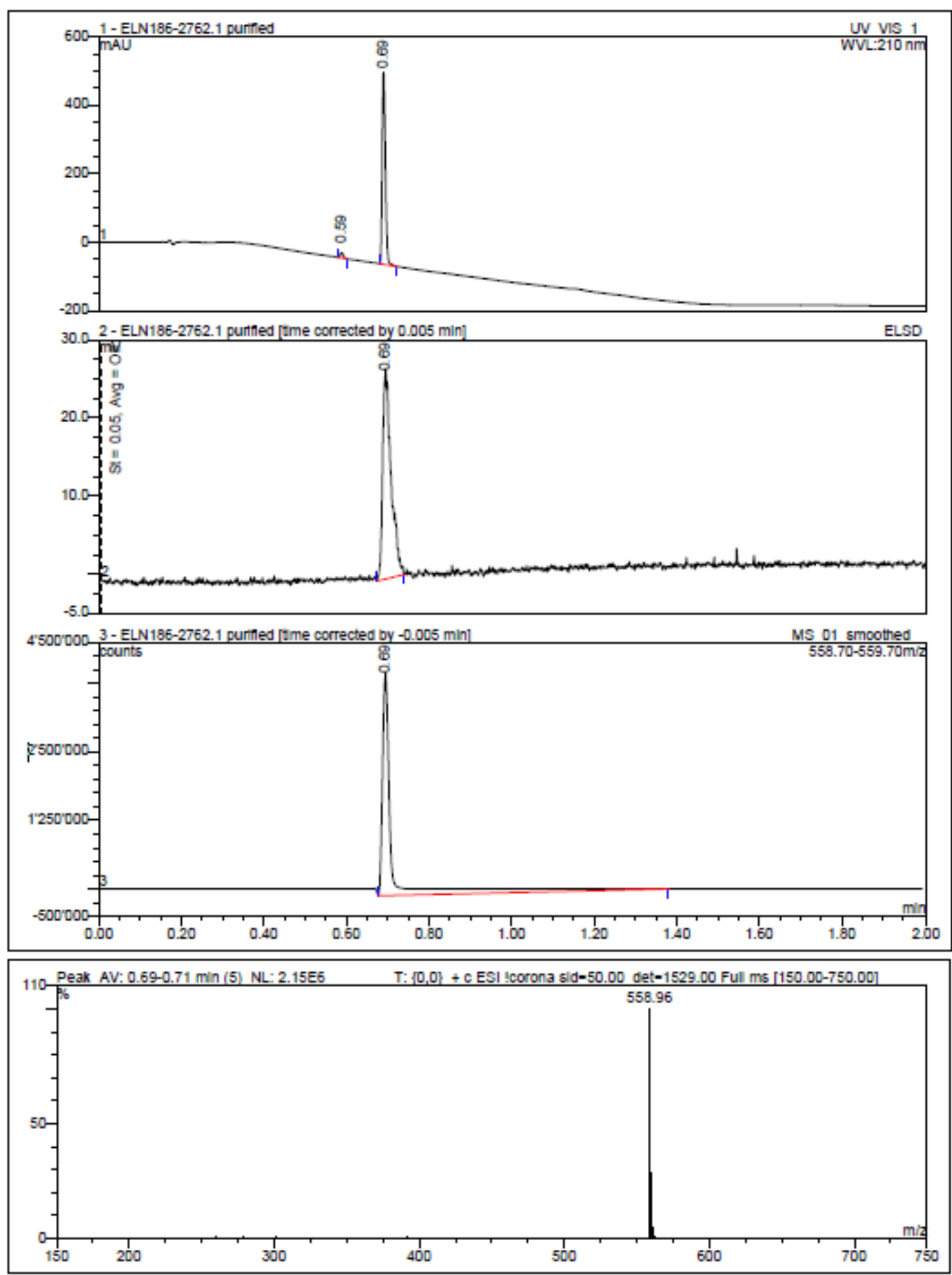

\begin{tabular}{|c|c|c|c|c|c|c|c|}
\hline $\begin{array}{l}\text { Peak No. } \\
\text { UV_VIS_2 }\end{array}$ & $\begin{array}{c}\text { Ret.Time } \\
\text { UV_VIS_2 } \\
\text { min }\end{array}$ & $\begin{array}{c}\text { Area } \\
\text { UV_VIS_2 }\end{array}$ & 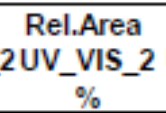 & $\begin{array}{c}\text { Mass 01 } \\
\text { UV_VIS_2 } \\
\text { amu }\end{array}$ & $\begin{array}{c}\text { Mass 02 } \\
\text { UV_VIS_2 } \\
\text { amu }\end{array}$ & $\begin{array}{c}\text { Mass 03 } \\
\text { UV_VIS_2 } \\
\text { amu }\end{array}$ & $\begin{array}{l}\text { UV Match } \\
\text { UV_VIS_2 }\end{array}$ \\
\hline 1 & 0.59 & 81 & 2.1 & 560.96 & 561.96 & 391.17 & 999 \\
\hline 2 & 0.69 & 3679 & 97.9 & 558.96 & 559.98 & 560.95 & 1000 \\
\hline Total: & & & 100.0 & & & & \\
\hline
\end{tabular}


Compound 28n:
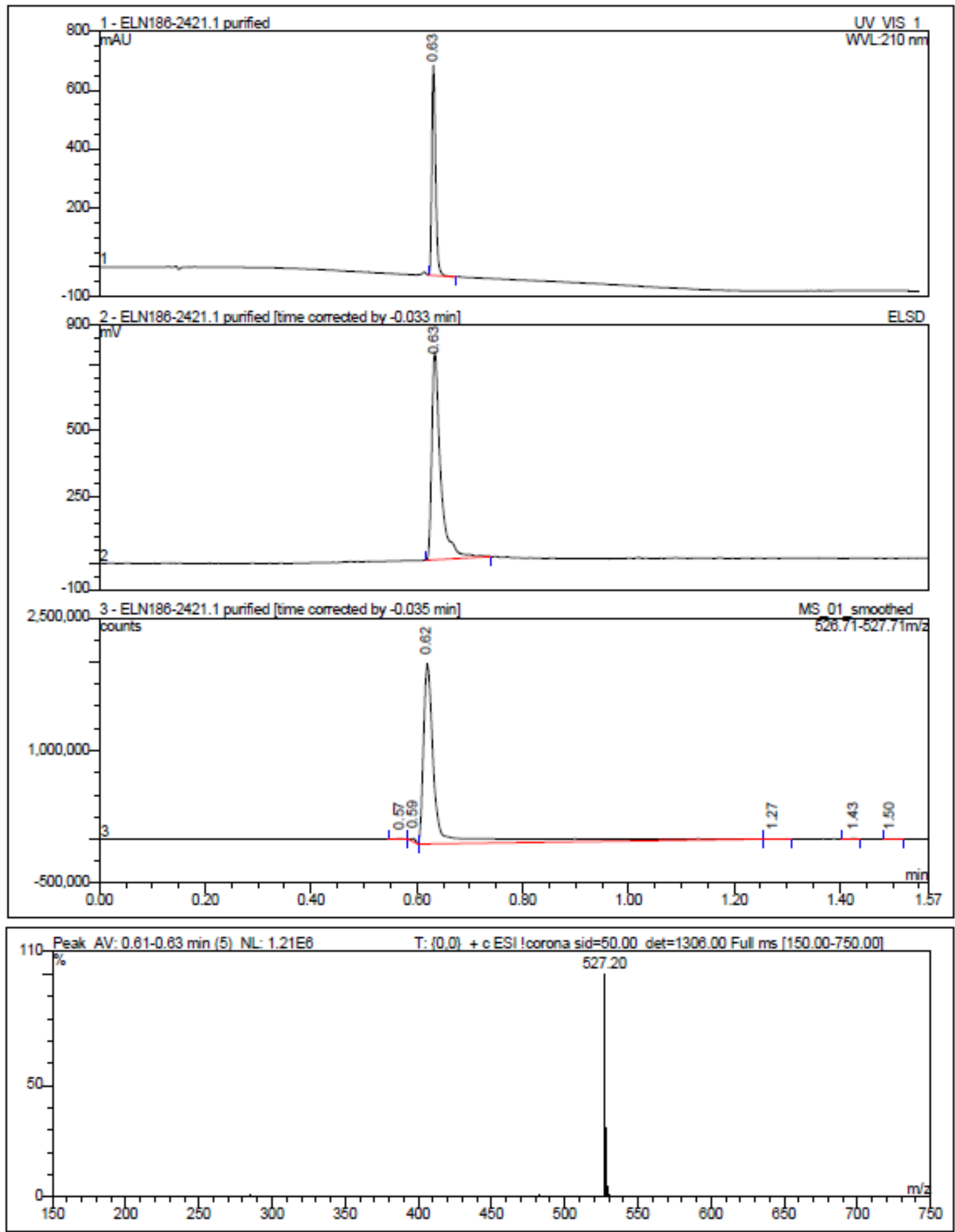
Compound 280:
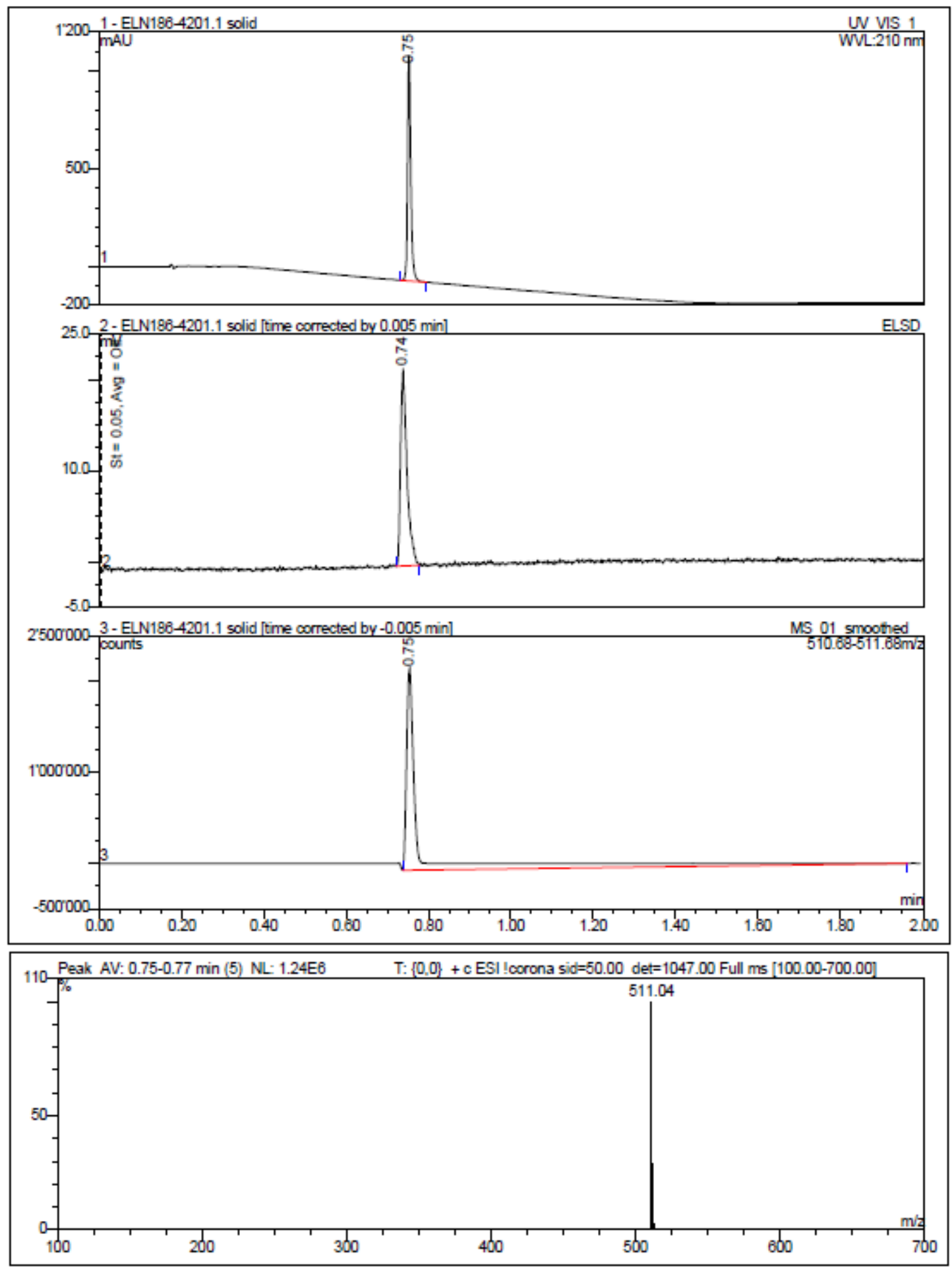
Compound 29:
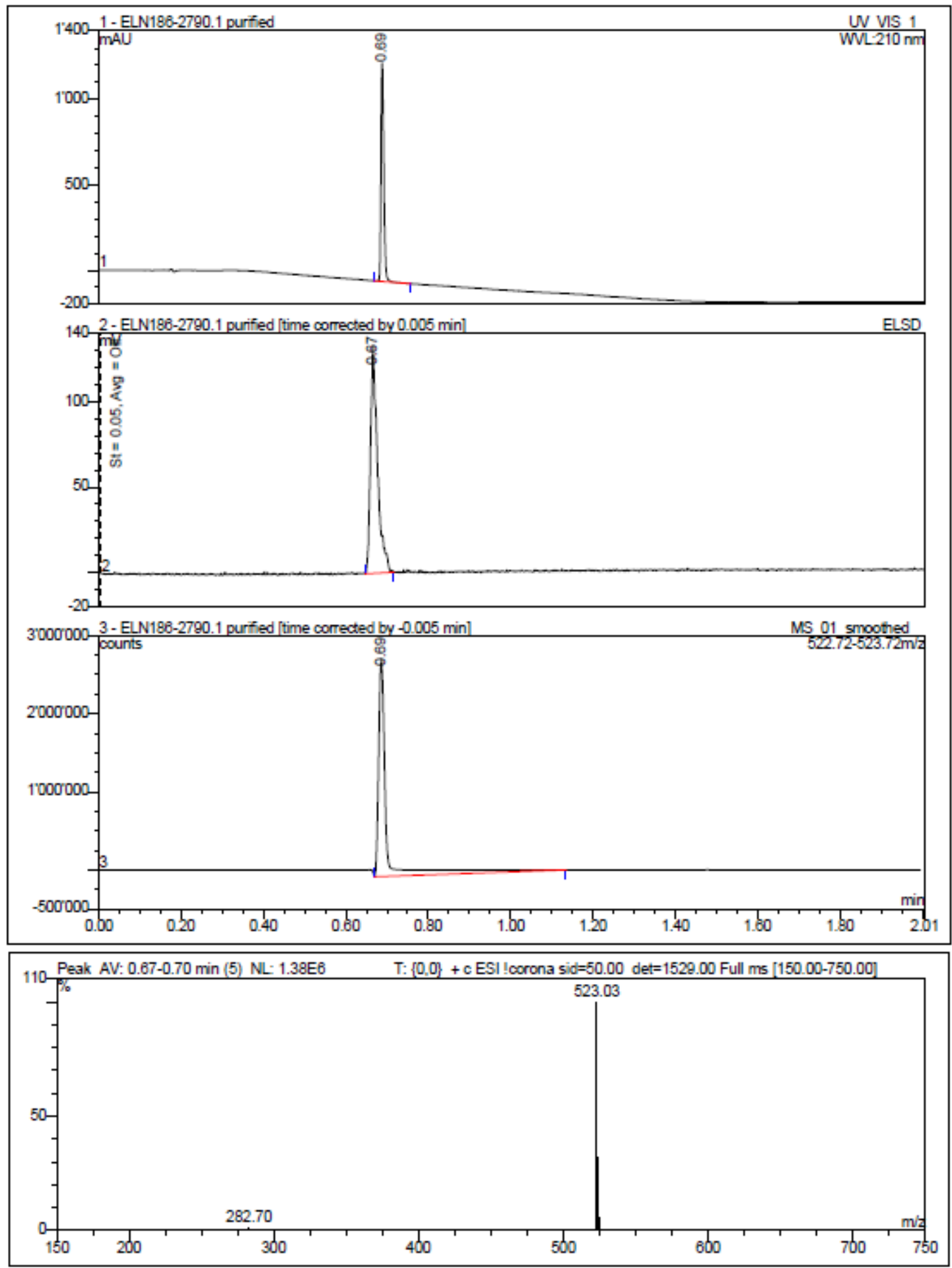
Compound 30:
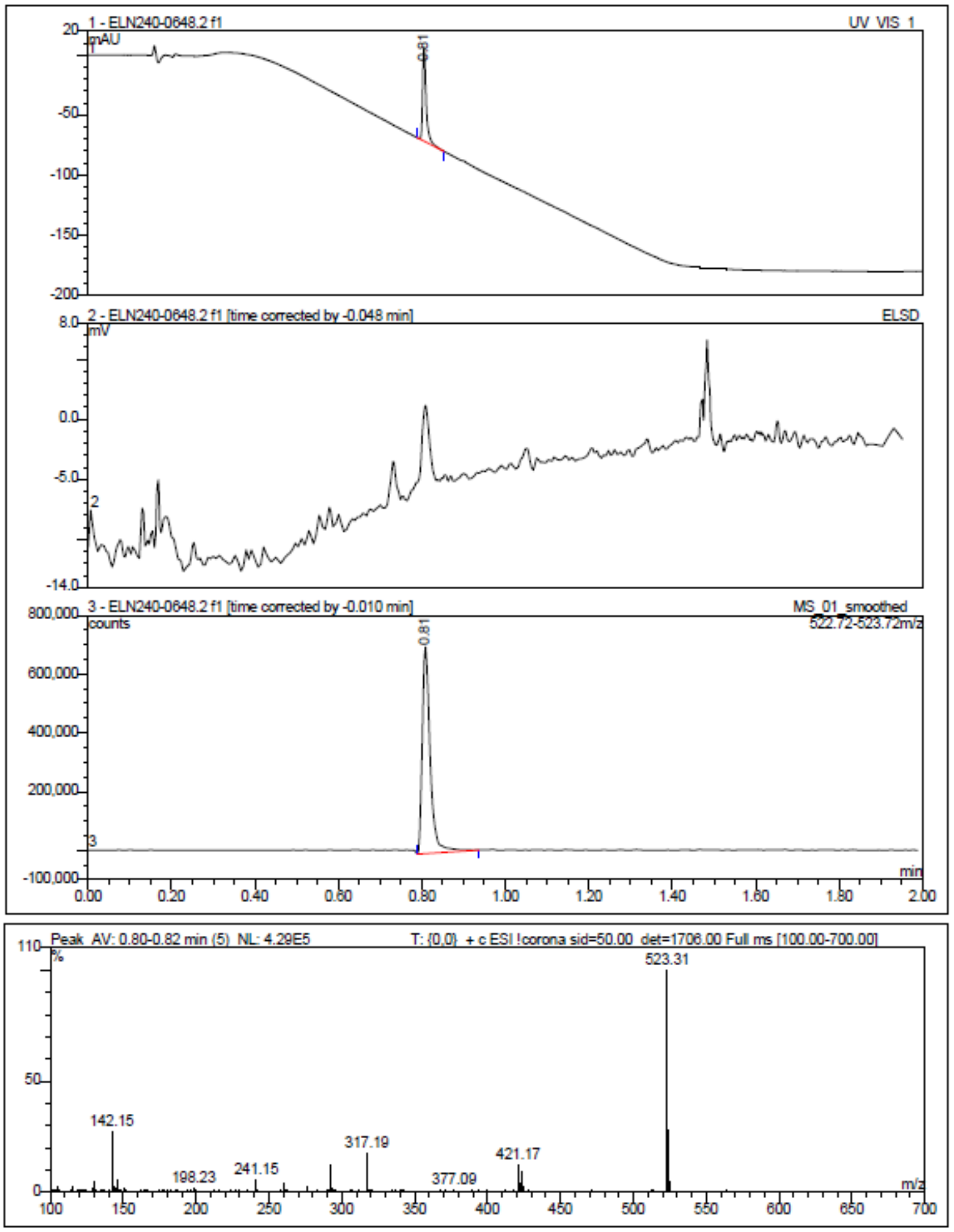
Compound 31:
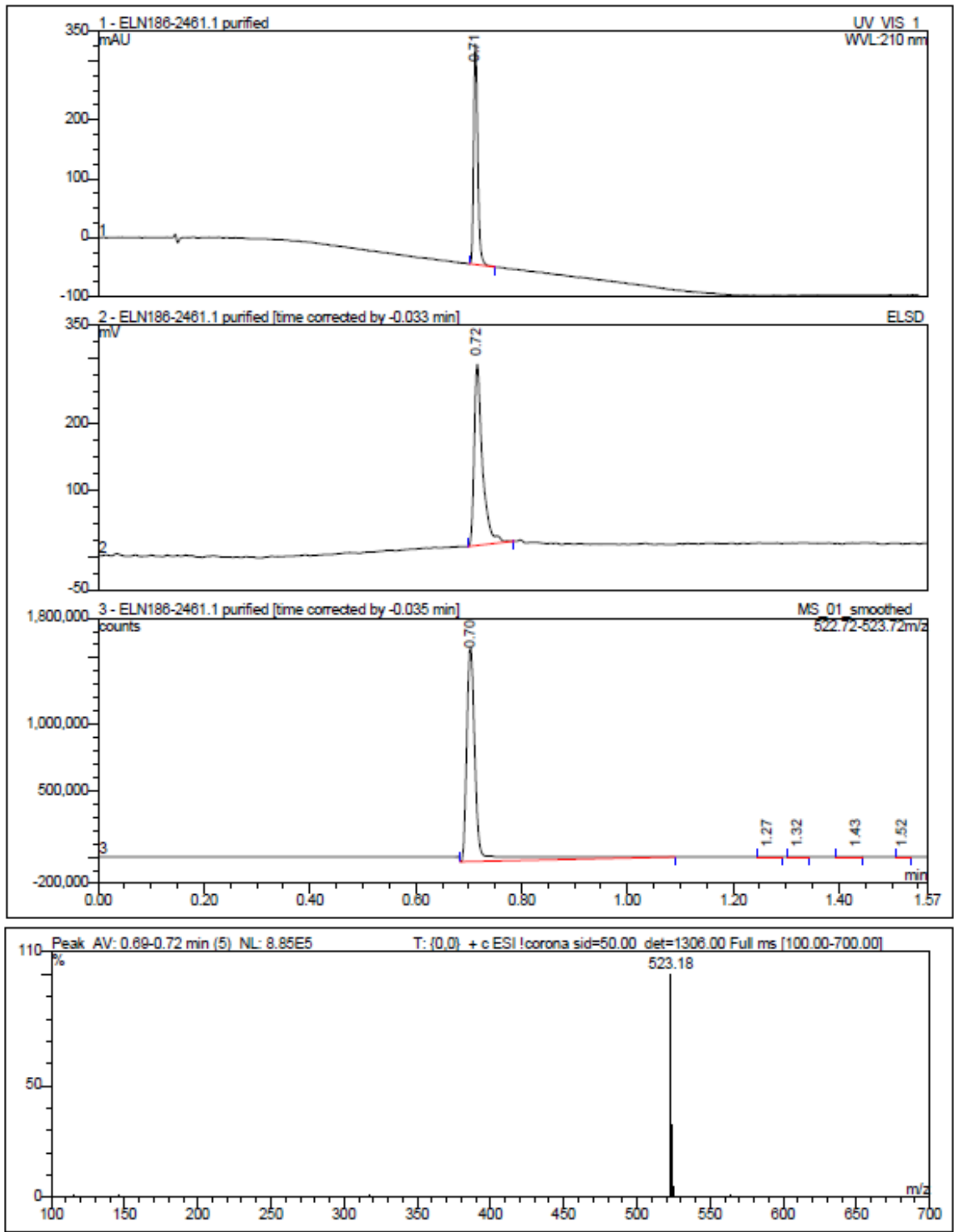